\title{
Technological innovations in diagnosis and treatment of glioblastoma
}

Citation for published version (APA):

Compter, I. (2021). Technological innovations in diagnosis and treatment of glioblastoma. [Doctoral Thesis, Maastricht University]. Maastricht University. https://doi.org/10.26481/dis.20211013ic

Document status and date:

Published: 01/01/2021

DOI:

10.26481/dis.20211013ic

Document Version:

Publisher's PDF, also known as Version of record

\section{Please check the document version of this publication:}

- A submitted manuscript is the version of the article upon submission and before peer-review. There can be important differences between the submitted version and the official published version of record.

People interested in the research are advised to contact the author for the final version of the publication, or visit the DOI to the publisher's website.

- The final author version and the galley proof are versions of the publication after peer review.

- The final published version features the final layout of the paper including the volume, issue and page numbers.

Link to publication

\footnotetext{
General rights rights.

- You may freely distribute the URL identifying the publication in the public portal. please follow below link for the End User Agreement:

www.umlib.nl/taverne-license

Take down policy

If you believe that this document breaches copyright please contact us at:

repository@maastrichtuniversity.nl

providing details and we will investigate your claim.
}

Copyright and moral rights for the publications made accessible in the public portal are retained by the authors and/or other copyright owners and it is a condition of accessing publications that users recognise and abide by the legal requirements associated with these

- Users may download and print one copy of any publication from the public portal for the purpose of private study or research.

- You may not further distribute the material or use it for any profit-making activity or commercial gain

If the publication is distributed under the terms of Article $25 \mathrm{fa}$ of the Dutch Copyright Act, indicated by the "Taverne" license above, 


\section{Technological innovations}

in diagnosis and treatment of glioblastoma

Inge Compter 


\section{ISBN:}

978-90-9034643-4

\section{Layout \& Cover design:}

Yasmine Medjadji, YM Design

\section{Production:}

Proefschriftmaken.nl

Part of the research in this thesis was financially supported by the Limburg University Fund/ SWOL and Stichting StopHersentumoren.nl

(C) I. Compter, Maastricht 2021. All rights reserved 


\title{
Technological innovations in diagnosis and treatment of glioblastoma
}

\author{
Proefschrift
}

ter verkrijging van de graad van doctor aan de Universiteit Maastricht, op gezag van de Rector Magnificus, Prof. dr. Rianne M. Letschert volgens het besluit van het College van Decanen, in het openbaar te verdedigen op woensdag

13 oktober 2021 om 16:00 uur

door

Inge Compter

geboren op 7 februari 1988

te Hoogeveen 


\section{Promotores}

Prof. dr. P. Lambin

Prof. dr. D. De Ruysscher

\section{Co-promotores}

dr. D.B.P. Eekers

dr. A. Hoeben

Prof.dr. ir. A.L. Hoffmann (Universitätsklinikum Carl Gustav Carus Dresden/ Technische Universität Dresden)

\section{Beoordelingscommissie}

Prof. dr. F.J.W. Verhaegen (voorzitter)

Prof. dr. M. Lambrecht (UZ Leuven)

Prof. dr. M. Smits (Erasmus MC)

Prof. dr. Y.Temel

dr. F. de Vos (UMC Utrecht)

The work presented in this thesis was made possible by the financial support of:

Stichting Wetenschappelijk Onderwijs Limburg (SWOL) and a grant from Stichting StopHersentumoren.nl. 
voor Papa 

Chapter 1

General introduction

Part I Decision support systems

Chapter 2

Noninvasive Glioblastoma Testing: Multimodal Approach to Monitoring

and Predicting Treatment Response

Chapter 3

Deciphering the glioblastoma phenotype by Computed Tomography radiomics

Part II Ultra-high field Magnetic Resonance Imaging

Chapter 4

Technical feasibility of integrating $7 T$ anatomical MRI in image-guided

radiotherapy of glioblastoma: a preparatory study

Chapter 5

Characterizing geometrical accuracy in clinically optimized 7T

and 3T MR images for high-precision radiation treatment of brain tumours

Part III Autophagy inhibition

Chapter 6

Chloroquine combined with concurrent radiotherapy and

temozolomide for newly diagnosed glioblastoma: A phase IB trial

Chapter 7

General Discussion

Chapter 8

Future Perspectives

Summary

Impact paragraph

Dankwoord 189

Curriculum Vitae 



$\begin{array}{ll}\text { 3D-TFE } & \text { Turbo field echo } \\ \text { AE } & \text { Adverse events } \\ \text { BO } & \text { Static magnetic field strength } \\ \text { CPS } & \text { Clinical prognostic score } \\ \text { CT } & \text { Computed tomography } \\ \text { CTC } & \text { National Cancer Institute Common Toxicity Criteria } \\ \text { CQ } & \text { Chloroquine } \\ \text { DICOM } & \text { Digital Imaging and Communications in Medicine } \\ \text { DLT } & \text { Dose-limiting toxicities } \\ \text { DSV } & \text { Diameter of a spherical volume } \\ \text { EGFR } & \text { Epidermal growth factor receptor } \\ \text { FLAIR } & \text { Fluid attenuation inversion recovery } \\ \text { FOV } & \text { Field of view } \\ \text { GBM } & \text { Glioblastoma } \\ \text { GD } & \text { Geometrical distortion } \\ \text { GTV } & \text { Gross tumour volume } \\ \text { HCQ } & \text { Hydroxychloroquine } \\ \text { HU } & \text { Hounsfield Units } \\ \text { IDH1 } & \text { Isocitrate dehydrogenase (NADP(+)) 1 } \\ \text { IBSI } & \text { Image Biomarker Standardization Initiative } \\ \text { KM } & \text { Kaplan-Meier } \\ \text { LASSO } & \text { least absolute shrinkage and selection operator } \\ \text { LR } & \text { Left-right } \\ \text { MAD } & \text { Mean absolute deviation } \\ \text { MGMT } & \text { O-6-methylguanine-DNA methyltransferase } \\ \text { MP2RAGE } & \text { Magnetization-prepared rapid gradient-echo } \\ \text { MRI } & \text { Magnetic resonance imaging } \\ \text { MTD } & \text { Maximum tolerated dose } \\ \text { OS } & \text { Overall survival } \\ \text { POI } & \text { Point-of-interest } \\ \text { po qd } & \text { Per os quaque die } \\ \text { PS } & \text { Performance status } \\ \text { RF } & \text { Radiofrequency } \\ \text { RPA } & \text { Recursive partitioning analysis } \\ \text { RPS } & \text { Radiomics prognostic score } \\ & \end{array}$


RTP Radiation therapy planning

SAE Serious adverse events

SD Standard deviation

SI Superior-inferior

SNR Signal-to-noise

T Tesla

T1-FFE Fast-field gradient echo

T1-GRE Multi-echo gradient echo

T2-SPACE T2-sampling perfection with application optimized contrasts using different flip angle evolution

T2-VISTA Volume isotropic Turbo spin echo

TMZ Temozolomide

TPS Treatment planning system

TRIPOD Transparent Reporting of a multivariable prediction model for Individual Prognosis Or Diagnosis

UHF Ultra-high field

VOI Volume-of-interest

VPS Volume-based prognostic score

WHO World Health Organization 
Chapter 1 - General introduction 
$-12$ 


\section{Glioblastoma}

Gliomas are the most common type of tumors originating in the central nervous system in both children and adult patients, with an annual incidence of approximately 1200 patients in the Netherlands ${ }^{1}$. They arise from the brain glial cells, which include astrocytes, oligodendrocytes and ependymal cells. Each type of glioma is currently classified based on correspondence with its presumed cell of origin, molecular features and aggressiveness (WHO classification) ${ }^{2}$. This thesis discusses innovations in both diagnostic imaging modalities and treatment of the most aggressive type of gliomas: glioblastoma (GBM); WHO grade IV.

Patients with GBM are presented with a dismal prognosis. Despite aggressive multimodality regimens including surgical resection, radiation therapy and chemotherapy the median overall survival (OS) is only 15 months and no more than $5 \%$ of patients survive up to 3 years after diagnosis ${ }^{3}$. Figure 1 shows a recent analysis of the survival outcome of patients with a GBM from a comprehensive, prospective population-based Quality Registry Neuro Surgery in The Netherlands between 2011 and $2014^{4}$. The value of post-operative radiation therapy has been demonstrated in several randomized controlled studies and has changed over time from whole-brain radiotherapy to more conformal radiotherapy in which the tumor and its surrounding marginal tissue are treated with modern intensity-modulated radiation therapy or volumetric-modulated arc therapy ${ }^{5}$. Temozolomide, an alkylating cytostatic agent, has become standard of care since 2005 after publication of the EORTC 26981/ 22981NCIC CE3 trial. This study was the first to unequivocally demonstrate survival benefit from the addition of chemotherapy to radiation therapy and adjuvant courses of temozolomide ${ }^{3}$.

In 2016 the World Health Organization Classification of Tumors of the Central Nervous System (CNS WHO) revised the classification of primary brain tumors and incorporated molecular parameters in addition to histology and grade to redefine tumor types, i.e. IDH mutation/wild type, ATRX loss, TP53 mutation, 1p/19q codeletion ${ }^{2}$. This classification provides an improved prediction of the prognosis when compared to histology and grade alone. GBM can be divided into IDH wild type, which represents approximately $90 \%$ of the cases and the IDH-mutant GBM ${ }^{2}$. The IDH wildtype, commonly referred to as "primary GBM", most often develops in patients over 55 years of age. In comparison, the IDH mutant or "secondary GBM" arises in younger patients and can dedifferentiate from WHO grade II or III glioma. 


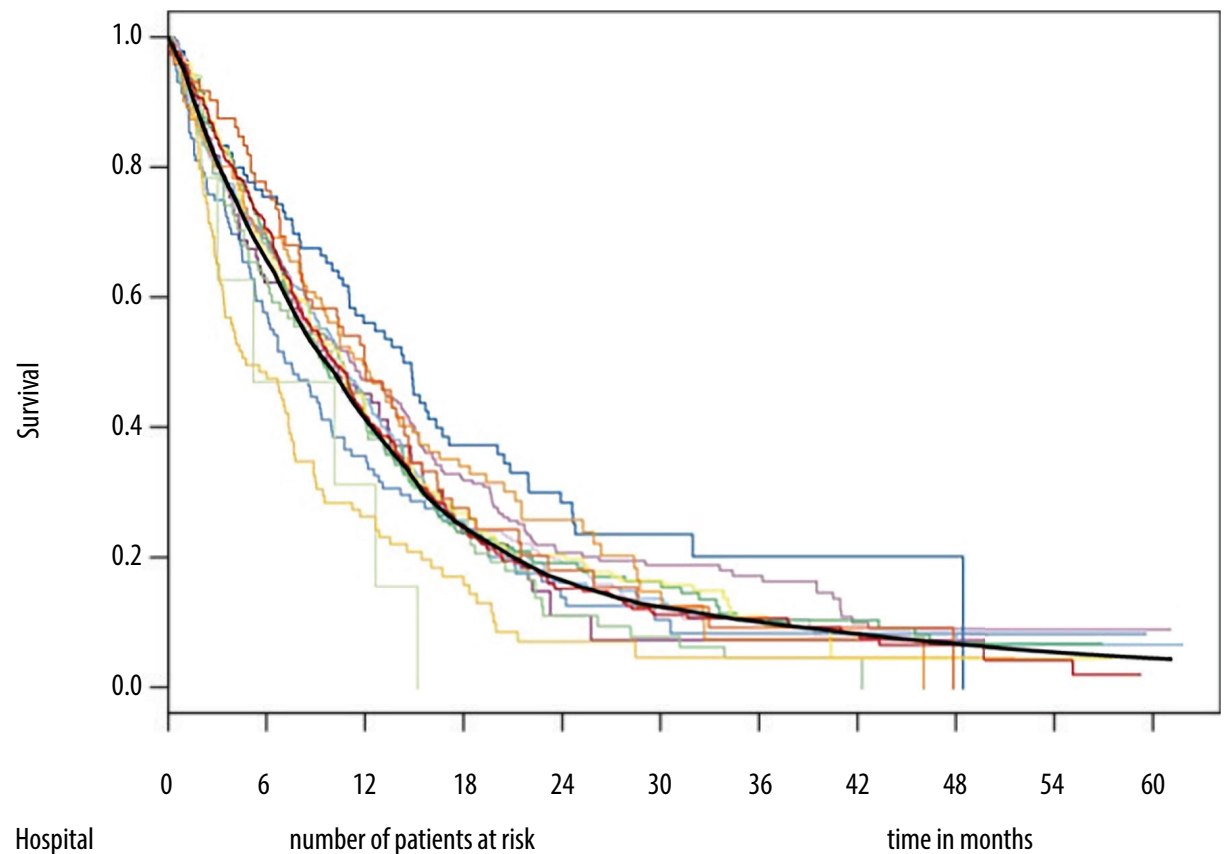

\begin{tabular}{|c|c|c|c|c|c|c|c|c|c|}
\hline 77 & 48 & 34 & 14 & 3 & 2 & 2 & 1 & 1 & 0 \\
\hline 228 & 155 & 107 & 65 & 36 & 28 & 20 & 11 & 9 & 4 \\
\hline 277 & 179 & 115 & 56 & 39 & 18 & 11 & 5 & 1 & 0 \\
\hline 95 & 67 & 47 & 27 & 18 & 9 & 3 & 2 & 1 & 0 \\
\hline 116 & 67 & 35 & 23 & 11 & 5 & 3 & 1 & 1 & 1 \\
\hline 268 & 170 & 112 & 57 & 30 & 19 & 13 & 8 & 3 & 3 \\
\hline 196 & 135 & 78 & 43 & 28 & 17 & 10 & 9 & 5 & 1 \\
\hline 82 & 51 & 34 & 20 & 7 & 5 & 2 & 0 & 0 & 0 \\
\hline 102 & 3 & 2 & 0 & 0 & 0 & 0 & 0 & 0 & 0 \\
\hline 232 & 155 & 93 & 56 & 33 & 22 & 6 & 2 & 2 & 2 \\
\hline 103 & 45 & 25 & 12 & 5 & 1 & 1 & 1 & 1 & 0 \\
\hline 111 & 77 & 53 & 30 & 16 & 6 & 1 & 1 & 0 & 0 \\
\hline 73 & 55 & 34 & 17 & 8 & 4 & 2 & 1 & 0 & 0 \\
\hline 348 & 240 & 142 & 74 & 37 & 24 & 18 & 8 & 4 & 2 \\
\hline
\end{tabular}

Fig. 1 Survival outcome over months as Kaplan-Meier curves per hospital in colors and overall survival function in black based on the Cox regression model with riskstandardization for age, performance, and treatment year. Data from 2,409 adults with first-time glioblastoma surgery at 14 Dutch hospitals obtained from the Quality Registry Neuro Surgery in The Netherlands between 2011 and 2014. Adapted from De Witt Hamer et $a l^{4}$

The symptoms of patients with a GBM depend on both the tumor's size and location within the brain. As GBM proliferate very rapidly, symptoms usually develop within weeks before diagnosis. The most frequent presenting symptoms include headaches, due to accompanying edema and a rise in intracranial pressure ( 60\%), seizures $(\sim 25 \%)$, focal neurological deficits, i.e. loss of vision, motor or sensory deficits and cognitive disturbances or personality changes $(10-40 \%)^{6}$. 
The prognosis for patients with a GBM has not improved significantly over the last 15 years, despite improvements in diagnostic and therapeutic strategies and multiple clinical trials with targeted drugs, anti-angiogenic compounds or immune modulators (e.g. immune checkpoint inhibitors, vaccinations) ${ }^{7-11}$. The current standard of care still comprises the same treatment schedule, consisting of consecutive i.e., surgical resection followed by concurrent radiotherapy and temozolomide ${ }^{3}$. One of the reasons GBM recur in almost all patients is that GBM encompass the entire brain, due to an infiltrative growth pattern and spread along white matter tracts. Microscopic tumor invasion is being underestimated by conventional imaging techniques and therefore a complete resection or radiation treatment to a high dose of the entire tumor volume is virtually impossible ${ }^{12,13}$. Additionally, it has been shown that inter-and intratumoral molecular heterogeneity is an important reason for treatment failure ${ }^{14}$. Optimizing diagnostic modalities to predict the molecular GBM heterogeneity and aggressiveness/invasiveness might eventually result in tumor tailored treatment plans. This thesis discusses innovations in both diagnostic imaging and treatment of GBM, and how to implement these advancements into clinical workflows and personalized therapy approaches, which can hopefully help bring about a sorely needed improved prognosis for these patients.

\section{Structural and functional imaging of glioblastoma}

Imaging is an essential part of the diagnosis, treatment response and follow-up of GBM. Upon presentation, most patients initially undergo computed tomography (CT) which is both relatively fast, cheap and widely available ${ }^{15}$. Moreover, during the course of radiation treatment $\mathrm{CT}$ scans are standard of care for radiation treatment planning (RTP) ${ }^{16}$ as they offer 1) Hounsfield units (HU) which can be transformed into electron densities, necessary for dose calculations, 2) provide high spatial fidelity, i.e. no geometrical distortions, and 3) offer a clear visualization of bony landmarks such as the skull base necessary for accurate positioning during radiation treatment.

However, for diagnostic and surgical/radiation treatment planning purposes magnetic resonance imaging (MRI) offers a superior structural image quality due to its high soft-tissue contrast ${ }^{17}$. This provides improved anatomic detail of normal brain structures and detection of tumor-infiltrated regions. Conventional MRI pulse sequences for evaluation of intracranial abnormalities include native T1-weighted (T1w), T2-weighted (T2w), fluid-attenuated inversion recovery (FLAIR), T2*-weighted $\left(\mathrm{T}^{*} \mathrm{~W}\right)$ gradient echo and post-contrast T1 $\mathrm{w}$ images. The addition of a gadoliniumbased contrast agent can provide information with regard to tumor vascularization and the blood-brain barrier (BBB) essential to the accurate radiological diagnosis and delineation of a GBM ${ }^{18}$. Advanced multi-parametric functional MRI techniques such as dynamic susceptibility contrast (DSC), dynamic contrast enhancement (DCE), diffusion tensor imaging (DTI), and MR spectroscopy (MRS) can provide additional information in order to differentiate between vasogenic edema and infiltrating tumor cells ${ }^{19}$. In addition to these advanced sequences MRI scanners with ultra-high 
field (UHF) strengths, having a main magnetic field (B0) strength of 7 Tesla (T) or greater, have become available for clinical research in the past years. These ultra-high field strengths result in a higher contrast-to-noise ratio compared to conventional high-field strength scanners (having a B0 field strength of $1.5 \mathrm{~T}$ or $3 \mathrm{~T}$ ) that are widely available in diagnostic radiology ${ }^{20}$. However, the benefit of UHF-MRI for the diagnosis, treatment and follow-up of GBM needs to be demonstrated in clinical trials.

Positron emission tomography (PET) is increasingly implemented into clinical practice in order to provide insight into physiological activities (e.g. metabolic processes) of tissues and treatment response of gliomas in particular. The Response Assessment in Neuro-Oncology (RANO) working group together with the European Association for Neuro-Oncology have published recommendations on the application of PET in the management of patients with gliomas ${ }^{21}$. Main indications for PET imaging in glioma with radiolabeled amino acids such as ${ }^{11} \mathrm{C}$-methionine, ${ }^{18} \mathrm{~F}$-FDOPA, and ${ }^{18} \mathrm{~F}$-FET include the differentiation of neoplastic versus non-neoplastic tissue, "hot-spot" localization for biopsy planning, prognostication and diagnosis of treatment-induced changes versus true tumor progression.

\section{Radiation treatment planning}

CT scans are acquired prior to the course of radiation treatment for dose calculation and optimization purposes. However, as stated above, MRI has a superior anatomical image quality in comparison to CT due to its high soft-tissue contrast, which is required for the delineation of the central nervous system organs at risk and the tumor. In order to define the treatment volume, a pre-treatment MRI scan is coregistered to the pre-treatment $C T$ scan, after which the latter is used as a reference image. The gross tumor volume (GTV) is the visible tumor on both MRI and CT and is delineated according to the ESTRO-ACROP guidelines ${ }^{22}$. The GTV is defined as the resection cavity (if present) plus any enhancing tumor on contrast-enhanced T1weighted MRI, preferably $1 \mathrm{~mm}$ slides. The clinical target volume (CTV) includes the GTV plus a safety margin to account for microscopic tumor spread not visible on high-field strength MRI or CT. The CTV is generated by adding an isotropic margin of $1.5-2 \mathrm{~cm}$ to the GTV and adjusted to the anatomical borders such as the skull. Furthermore, the CTV may be adjusted to include abnormal FLAIR signal suggestive for microscopic spread up to $2 \mathrm{~cm}$ in the Dutch National protocol. Additionally, the CTV is extended with a planning target volume (PTV) margin that accounts for uncertainties in treatment planning such as patient setup inaccuracies, intrafractional motion, errors in multi-modality image registration, target volume delineation and anatomical changes ${ }^{23,24}$. This margin is based on institutional measurements and can differ between departments and usually varies between 1-5mm. An increase in uncertainties results in an increased margin (and hence radius of the PTV) that translates into an expansion of the treated volume that is proportional to the third power of the radius ${ }^{25}$.

As the risk of toxicity increases with a larger treatment volume, maximum effort is 
required to keep PTV margin as small as possible. Figure 2 shows the aforementioned treatment volumes applied to a patient with a GBM.
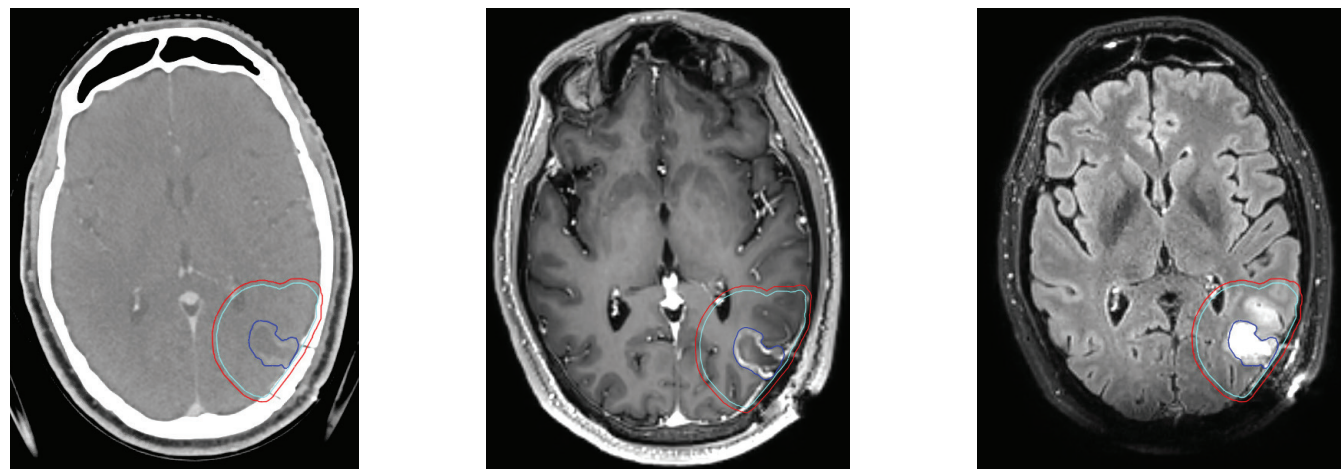

Fig. 2 Example of different volumes delineated on a CT (left), transversal contrastenhanced T1W 3T MR (middle) and transversal native FLAIR 3T MR (right) image of a patient with a GBM in the parieto-occipital lobes on the left side after a resection. The gross tumor volume (GTV) is delineated in dark blue, the clinical target volume (CTV) in cyan and the planning target volume (PTV) in red. The CTV was created by adding a 1.5 $\mathrm{cm}$ isotropic margin around the GTV and includes the abnormal FLAIR signal up to $2 \mathrm{~cm}$ from the GTV. The CTV was then adjusted to the anatomical boundaries. A PTV margin of $2 \mathrm{~mm}$ was added to the CTV

\section{Decision support systems}

In order to select patients that benefit from concurrent chemoradiation and an adjuvant course of temozolomide or offer best supportive care to those who don't computerized decision support systems are being developed ${ }^{26}$. These computer programs sift through and analyze massive amounts of medical data, e.g., clinical parameters, imaging features and biological data (e.g. molecular markers), compiling comprehensive information that can be used to solve problems in medical decision making. A method that is most frequently applied for clinical purposes is a recursive partitioning analysis ${ }^{27,28}$. The prognostic factors included in this model are age, WHO performance status, extent of surgery, and the Mini-Mental Status Examination.

In recent years, there has been an increased interest in radiological features of GBM. One of the methods to characterize the glioma phenotype is through qualitative assessment of these features on MRI such as tumor volume, contrast enhancement, presence of necrosis and high T2w MRI signal reflecting both edema and non-enhancing tumor ${ }^{17}$. In order to standardize the description of brain tumor morphology in imaging the "Visually AcceSsible Rembrandt Images (VASARI)" features were introduced. Several investigators have demonstrated a correlation between the VASARI features, prognosis and molecular subtype ${ }^{29-33}$. Major drawbacks of the systematic description of these features are that they are subject to large inter- 
and intra-observer variability and that the systematic description is time-consuming. Radiomics may overcome these limitations. Radiomics is a computerized method that extracts large amounts of features from radiographic medical images (e.g., CT, PET and MRI) through data-characterization algorithms ${ }^{34,35}$.

The radiomics hypothesis is that medical images contain quantifiable information on the pathophysiology of e.g. a tumor and that this information can be extracted. The process contains several steps including segmentation of the volume of interest e.g. the contrast-enhanced tumor on CT or MR images, followed by the extraction of a large amount of image features and the development of a model investigating a potential association with a predefined outcome.

The image features can be categorized in the following groups:

1. histogram of intensity values,

2. object shape and size,

3. texture

4. parameters of image filtering.

Based on these features radiomics can potentially capture the glioblastoma phenotype which may hold prognostic and predictive information, both of which can be added to the already known clinical and molecular information, and collectively be incorporated in a model in order to personalize and optimize treatment for patients with a GBM ${ }^{36,37}$.

\section{Molecular heterogeneity in glioblastoma}

Large-scale genomic studies have demonstrated that GBM display large inter- and intratumoral molecular heterogeneity ${ }^{38}$. This poses major challenges for treatment as one tumor clone may be sensitive to treatment, whereas another one demonstrates treatment resistance. Intratumoral heterogeneity is based on at least three main determinants:

1. the presence of subclonal mutations, arising during tumor evolution,

2. epigenetic and developmental programs such as those arising from glioma stem cells (GSCs)

3. environmental factors such as hypoxia and nutrient depriviation ${ }^{39}$. GSCs are known to be tumorigenic in contrast to the rest of the tumor which usually consists of differentiated cells with low tumor-forming capability ${ }^{40}$. Key features of GSC are sustained self-renewal, persistent proliferation and the ability to facilitate tumor initiation when implanted in the cortex of rodents. Based on a transcriptomic and genomic analysis GBM can be divided into four distinct subtypes: classical, neural, proneural, and mesenchymal ${ }^{41}$. The classical subtype demonstrates an amplification of chromosome 7, loss of chromosome 10, EGFR amplification, NES expression and $C D K N 2 A$ deletion; the neural subtype shows expression of neural markers GABRA1, $S L A 12 A 5, S Y T 1$, and $N E F L$, the proneural subtype displays point mutations in $I D H 1$, alterations in PDGFR and mutations in TP53, DLL3, DCX, TCF4, SOX, ASCL1, and OLIG2, whereas the mesenchymal subtype demonstrates loss of $C D K N 2 A$, high expression of SERPINE1, TRADD, RELB, and CTGF, loss of NF1 and expression of mesenchymal markers 
CHI3L1, MET, CD44, and MERTK. These genetic changes can be important for survival prediction as there are indications that the mesenchymal subtype displays the worst prognosis, while the proneural has a more favourable outcome ${ }^{42}$.

Single-cell RNA sequencing techniques are able to measure the transcriptional output of tumor cells which reflects the intratumoral heterogeneity ${ }^{39}$. However, the amountand molecular differentiation of the different clones present in a specific GBM is currently being underestimated, due to the fact that 1) single cell RNAseq is currently not part of the standard of care and 2) gross total resection is not always feasible. In addition, intratumoral heterogeneity cannot yet be measured in a reliable and noninvasive way with the currently available imaging techniques such as MRI and PETimaging. Therefore, targeted treatment of different tumor clones may not be feasible, also considering the toxicity of combining different targeted treatments eg. TKIs. It may therefore be more successful to consider other targets such as targeting GSC, regulate the tumor microenvironment or inhibit metabolic pathways (e.g. autophagy).

\section{Autophagy}

Amplification or mutation of the epidermal growth factor receptor (EGFR) gene is observed in a number of different cancers including GBM. It is thought to be a major contributor to radio resistance at least partially caused by enhanced repair of DNA double strand breaks ${ }^{43}$. The EGFR mutation type III variant (EGFRvIII) is observed in approximately $25-30 \%$ of GBM patients and stimulates growth and promotes survival under stress conditions such as nutrient deprivation and hypoxia ${ }^{44,45}$. EGFRvIII has also been observed in GSCs 46. The presence of EGFRvIll is associated with a poor prognosis in $\mathrm{GBM}^{47,48}$. Malignant cells survive stress conditions by activation of a cell survival mechanism called autophagy through the expression of EGRFvIll. Autophagy, a lysosomal degradation pathway, is essential to maintain a viable hypoxic fraction ${ }^{49}$. It allows cells to recycle nutrients in order to maintain energy levels, protein synthesis and metabolic processes resulting in resistance to both radiotherapy and chemotherapy. The lysomotropic drug Chloroquine (CQ) inhibits autophagy by blocking its final degradative step. This results in a reduction of hypoxic regions within a tumor, making the cells more susceptible to radiation treatment ${ }^{50}$. This potential clinical effect has been demonstrated in a small randomized controlled trial in which CQ was added to radiation therapy and Carmustine for patients with a GBM. Even though not statistically significant the rate of death with time was approximately half as large in patients receiving CQ when compared to placebo ${ }^{51}$. Preclinical evidence has shown that although an effect was observed in all tumors, EGFRvIll positive GBM are more dependent on autophagy for survival and that these patients may benefit most from the addition of $\mathrm{CQ}^{52}$. However, irrefutable evidence for the effects of $\mathrm{CQ}$ in combination with radiation therapy and temozolomide (TMZ), the current standard treatment, stills needs to be presented. 


\section{Objectives of this thesis}

As stated before GBM has so far remained an incurable disease with a very poor prognosis despite an extensive range of treatment options. The cause is supposed to include multifactorial aspects that include at least: the infiltrative growth pattern of GBM and the inability of imaging modalities to visualize the tumor spread resulting in needlessly damaging healthy brain tissue, either surgically or with radiation treatment while leaving viable tumor cells alive, and biological factors comprising tumor heterogeneity, treatment resistance of glioma stem cells, and metabolic pathways that allow cell survival and proliferation under stress conditions such as radio- and chemotherapy, nutrient starvation and hypoxia.

The overall objectives of the work described in this thesis are:

1. Review the established and future diagnostic imaging methods and techniques used for the prediction of treatment response and the optimization of decision support systems to improve outcome prediction in patients with a GBM.

2. Investigate the technical feasibility to incorporate UHF MRI into neurosurgical navigation and radiation treatment planning systems for patients with GBM.

3. Determine the recommended phase II dose for $C Q$ in combination with concurrent radiotherapy with $T M Z$ in patients with a newly diagnosed GBM.

Firstly, it is hypothesized that a new decision support system can be constructed using a model that integrates a CT imaging based radiomics signature in order to improve the prediction of OS of patients with a GBM in comparison to the current clinically applied prediction models.

With regard to the second objective, it is hypothesized that integration of anatomically optimized 7T MRI into radiation treatment planning is technically feasible and fulfills the spatial accuracy requirements currently imposed on $3 \mathrm{TMRI}$ for the same purpose.

Finally, it is hypothesized that autophagy is one of the main reasons for radiation treatment resistance in GBM and that $\mathrm{CQ}$, as an autophagy inhibitor, can safely be combined with concurrent chemoradiation with TMZ in doses up to $600 \mathrm{mg}$ daily. 


\section{Outline of this thesis}

This thesis includes three parts that relate to different aspects of the diagnosis and treatment of GBM (Figure 3). Part I considers methods to monitor and predict treatment response which can be included in decision support systems in order to provide optimal treatment. Part II discusses relevant technical aspects of 7 T MRI for GBM and the necessary steps to integrate this into radiation treatment planning. In Part III treatment aspects, specifically the safety of autophagy inhibition through the addition of CQ in a phase Ib trial are discussed.

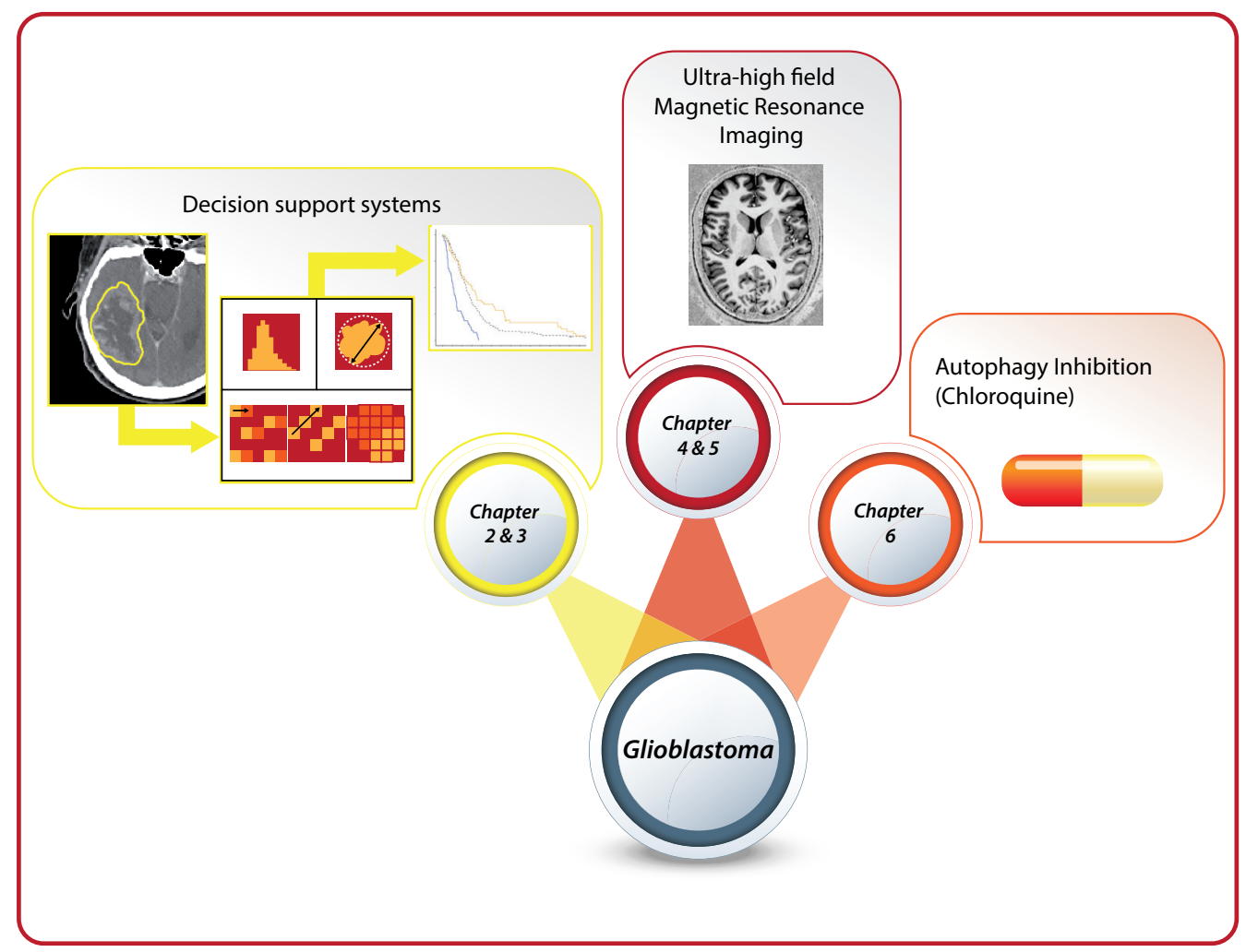

Fig. 3 Schematic overview of the outline of this thesis

Chapter 2 provides a comprehensive review of the techniques currently available to capture the heterogeneity of GBM including advanced MRI, nuclear imaging, liquid biopsy, and new integrated approaches such as radiogenomics and radiomics. This review is the first to combine these diagnostic methods into a noninvasive glioblastoma testing (NIGT) platform which can be used to evaluate the tumor as a whole and provides information on molecular characteristics and the tumor microenvironment. It can guide clinicians in selecting the optimal treatment option and follow-up for patients with a GBM. Furthermore, the review provides guidance for future research to determine the sensitivity, specificity and validation of the techniques discussed. 
Chapter 3 reports on the results of a retrospective analysis investigating the prognostic value of $C T$ imaging based radiomics when correlated with OS in patients with a GBM. Clinical decisions in GBM have primarily been based upon clinical factors and more recently on molecular markers. So far only a few radiomics studies have been published, mainly focussing on GBM features extracted from MR images. The current study demonstrates the feasibility of CT-based radiomics and assesses its prognostic value against known prognostic clinical factors in GBM. It furthermore shows the need for external validation and standardization of imaging protocols.

Chapter 4 reports on a preparatory study that investigates the technical feasibility of incorporating 7TMRI into neurosurgical navigation and radiation treatment planning systems. This study was performed in preparation of a prospective trial evaluating the high-resolution soft-tissue imaging of microvascularization in GBM (clinicaltrials. gov NCT02062372). It is the first study to specify an image transfer and processing workflow for $7 \mathrm{~T} \mathrm{MRI}$ to be included in such systems. Moreover, the study provides a structured qualitative and quantitative image quality analysis that is required before 7 T MRI can be implemented in radiation therapy planning with clinically acceptable spatial accuracy.

Chapter 5 focuses further on the quantitative image analysis of $7 \mathrm{~T}$ MRI for clinical application in radiation treatment planning and compares these results to both standard 3 T MR and CT imaging in brain tumors. This chapter shows that the systemand phantom/patient-related geometrical distortion of $7 \mathrm{~T} \mathrm{MRI} \mathrm{remains} \leq 1 \mathrm{~mm}$ in central regions of the brain. In the peripheral regions the geometrical distortion was larger and the PTV should be adjusted accordingly if UHF-MRI is implemented into radiation treatment planning.

In Chapter 6 presents the results from the phase Ib CQ trial. CQ has previously shown promising (pre-)clinical activity in GBM. However, the effects of CQ are potentially dose-dependent and the safety and efficacy in combination with concurrent chemoradiation with TMZ has not yet been investigated. It demonstrates that the addition of $C Q$ to chemoradiation can result in significant toxicity such as electrocardiogram QTc prolongation, blurred vision and nausea and that 200 $\mathrm{mg} C \mathrm{C}$ is the recommended phase II dose in newly diagnosed GBM. Moreover, an exploratory analysis confirms that EGFRvIII is a potential predictive biomarker for the response to CQ.

The conclusions and future perspectives of this thesis are discussed in Chapter 7. 
1. IKNL DBTR. Glioomzorg in Nederland. 2019.

2. Louis DN, Perry A, Reifenberger G, et al. The 2016 World Health Organization Classification of Tumors of the Central Nervous System: a summary. Acta neuropathologica. 2016;131(6):803-820.

3. Stupp R, Hegi ME, Mason WP, et al. Effects of radiotherapy with concomitant and adjuvant temozolomide versus radiotherapy alone on survival in glioblastoma in a randomised phase III study: 5-year analysis of the EORTC-NCIC trial. Lancet Oncol. 2009;10(5):459-466.

4. De Witt Hamer PC, Zwinderman $A H$, Ackermans $L$, et al. Between-hospital variation in mortality and survival after glioblastoma surgery in the Dutch Quality Registry for Neuro Surgery. Journal of neuro-oncology. 2019;144(2):313-323.

5. Laperriere N, Zuraw L Fau - Cairncross G, Cairncross G. Radiotherapy for newly diagnosed malignant glioma in adults: a systematic review. Radiother, Oncol. 2002(0167-8140).

6. Chang SM, Parney IF, Huang W, et al. Patterns of care for adults with newly diagnosed malignant glioma. JAMA : the journal of the American Medical Association. 2005;293(5):557-564.

7. Taal W, Oosterkamp HM, Walenkamp AM, et al. Single-agent bevacizumab or lomustine versus a combination of bevacizumab plus lomustine in patients with recurrent glioblastoma (BELOB trial): a randomised controlled phase 2 trial. Lancet Oncol. 2014;15(9):943-953.

8. Omuro A, Vlahovic G, Lim M, et al. Nivolumab with or without ipilimumab in patients with recurrent glioblastoma: results from exploratory phase I cohorts of CheckMate 143. Neuro-oncology. 2018;20(5):674-686.

9. Moughon D, Everson R, Odesa $S$, et al. ATIM-32. PHASE IIA CLINICAL TRIAL EVALUATING DENDRITIC CELL VACCINE FOR THE TREATMENT OF LOW-GRADE GLIOMAS. Neuro-oncology. 2016;18(suppl_6):vi25-vi25.

10. Hilf N, Kuttruff-Coqui $S$, Frenzel $K$, et al. Actively personalized vaccination trial for newly diagnosed glioblastoma. Nature. 2019;565(7738):240-245.

11. Wen PY, Weller M, Lee EQ, et al. Glioblastoma in Adults: A Society for Neuro-Oncology (SNO) and European Society of Neuro-Oncology (EANO) Consensus Review on Current Management and Future Directions. Neuro-oncology. 2020; [published online ahead of print, 2020 Apr 24].

12. Halperin EC, Bentel G, Heinz ER, Burger PC. Radiation therapy treatment planning in supratentorial glioblastoma multiforme: an analysis based on post mortem topographic anatomy with CT correlations. Int J Radiat Oncol Biol Phys. 1989;17(6):1347-1350. 
13. Sahm F, Capper D, Jeibmann A, et al. Addressing diffuse glioma as a systemic brain disease with single-cell analysis. Archives of neurology. 2012;69(4):523-526.

14. Mandel JJ, Yust-Katz S, Patel AJ, et al. Inability of positive phase II clinical trials of investigational treatments to subsequently predict positive phase III clinical trials in glioblastoma. Neuro-oncology. 2017;20(1):113-122.

15. Macdonald DR, Cascino TI Fau - Schold SC, Jr., Schold Sc Jr Fau - Cairncross JG, Cairncross JG. Response criteria for phase II studies of supratentorial malignant glioma. J Clin Oncol. 1990 Jul(0732-183X (Print)).

16. Olsen Dr Fau - Thwaites DI, Thwaites DI. Now you see it... Imaging in radiotherapy treatment planning and delivery. Radiother, Oncol. 2007 Nov(0167-8140 (Print)).

17. Rees J. Advances in magnetic resonance imaging of brain tumours. Curr Opin, Neurol. 2003(1350-7540).

18. Weinmann Hj Fau - Brasch RC, Brasch Rc Fau - Press WR, Press Wr Fau - Wesbey GE, Wesbey GE. Characteristics of gadolinium-DTPA complex: a potential NMR contrast agent. A J R Am J Roentgenol. 1984;Mar(0361-803X (Print)).

19. van Dijken BRJ, van Laar PJ, Holtman GA, van der Hoorn A. Diagnostic accuracy of magnetic resonance imaging techniques for treatment response evaluation in patients with high-grade glioma, a systematic review and meta-analysis. Eur, Radiol. 2017 Oct(1432-1084 (Electronic)).

20. Lupo JM, Li Y Fau - Hess CP, Hess Cp Fau - Nelson SJ, Nelson SJ. Advances in ultrahigh field MRI for the clinical management of patients with brain tumors. Curr Opin, Neurol. 2011 Dec(1473-6551 (Electronic)).

21. Albert NL, Weller M, Suchorska B, et al. Response Assessment in Neuro-Oncology working group and European Association for Neuro-Oncology recommendations for the clinical use of PET imaging in gliomas. Neuro, Oncol. 2016;Sep(1523-5866).

22. Niyazi M, Brada M, Chalmers AJ, et al. ESTRO-ACROP guideline "target delineation of glioblastomas". Radiother, Oncol. 2016;Jan(1879-0887).

23. van Herk M. Errors and margins in radiotherapy. Semin Radiat, Oncol. 2014; $\operatorname{Jan}(1053-4296)$.

24. Measurements TICoRUa. Prescribing, recording, and reporting photon-beam IMRT. Journal of the ICRU. 2010; Vol 10(1).

25. Verellen D, De Ridder M Fau - Linthout N, Linthout N Fau - Tournel K, Tournel K Fau - Soete G, Soete G Fau - Storme G, Storme G. Innovations in image-guided radiotherapy. Nat Rev, Cancer. 2007;Dec(1474-1768).

26. Walsh S, de Jong EEC, van Timmeren JE, et al. Decision Support Systems in Oncology. J C O Clin Cancer Inform. 2019 Feb(2473-4276 ). 
27. Mirimanoff RO, Gorlia T, Mason W, et al. Radiotherapy and temozolomide for newly diagnosed glioblastoma: recursive partitioning analysis of the EORTC 26981/22981NCIC CE3 phase III randomized trial. J Clin Oncol. 2006;24(16):2563-2569. Curran WJ, Jr., Scott CB, Horton J, et al. Recursive partitioning analysis of prognostic factors in three Radiation Therapy Oncology Group malignant glioma trials. Journal of the National Cancer Institute. 1993;85(9):704-710.

28. Gutman DA, Cooper LA, Hwang SN, et al. MR imaging predictors of molecular profile and survival: multi-institutional study of the TCGA glioblastoma data set. Radiology. 2013;267(2):560-569.

29. Gevaert O, Mitchell LA, Achrol AS, et al. Glioblastoma Multiforme: Exploratory Radiogenomic Analysis by Using Quantitative Image Features. Radiology. 2015;276(1):313.

30. Jain R, Poisson LM, Gutman D, et al. Outcome prediction in patients with glioblastoma by using imaging, clinical, and genomic biomarkers: focus on the nonenhancing component of the tumor. Radiology. 2014;272(2):484-493.

31. Nicolasjilwan M, Hu Y, Yan C, et al. Addition of MR imaging features and genetic biomarkers strengthens glioblastoma survival prediction in TCGA patients. Journal of neuroradiology Journal de neuroradiologie. 2015;42(4):212-221.

32. Mazurowski MA, Zhang J, Peters KB, Hobbs H. Computer-extracted MR imaging features are associated with survival in glioblastoma patients. Journal of neurooncology. 2014;120(3):483-488.

33. Aerts HJ, Velazquez ER, Leijenaar RT, et al. Decoding tumour phenotype by noninvasive imaging using a quantitative radiomics approach. Nature communications. 2014;5:4006.

34. Gillies RJ, Kinahan PE, Hricak H. Radiomics: Images Are More than Pictures, They Are Data. Radiology. 2016;278(2):563-577.

35. Kickingereder P, Gotz M, Muschelli J, et al. Large-scale Radiomic Profiling of Recurrent Glioblastoma Identifies an Imaging Predictor for Stratifying AntiAngiogenic Treatment Response. Clin Cancer Res. 2016;22(23):5765-5771.

36. Macyszyn L, Akbari H, Pisapia JM, et al. Imaging patterns predict patient survival and molecular subtype in glioblastoma via machine learning techniques. Neuro, Oncol. 2016;Mar(1523-5866 (Electronic)).

37. Patel AP, Tirosh I, Trombetta JJ, et al. Single-cell RNA-seq highlights intratumoral heterogeneity in primary glioblastoma. Science (New York, NY). 2014;344(6190):1396-1401.

38. Suva ML, Tirosh I. Single-Cell RNA Sequencing in Cancer: Lessons Learned and Emerging Challenges. Molecular cell. 2019;75(1):7-12. 
39. Reya T, Morrison Sj Fau - Clarke MF, Clarke Mf Fau - Weissman IL, Weissman IL. Stem cells, cancer, and cancer stem cells. Nature. 2001;414(6859):105-111.

40. Verhaak RG, Hoadley Ka Fau - Purdom E, Purdom E Fau - Wang V, et al. Integrated genomic analysis identifies clinically relevant subtypes of glioblastoma characterized by abnormalities in PDGFRA, IDH1, EGFR, and NF1. Cancer Cell. 2010;17(1):98-110.

Brennan CW, Verhaak Rg Fau - McKenna A, McKenna A Fau - Campos B, et al. The somatic genomic landscape of glioblastoma. Cell. 2013;155(2):462-477.

41. Chakravarti A, Dicker A Fau - Mehta M, Mehta M. The contribution of epidermal growth factor receptor (EGFR) signaling pathway to radioresistance in human gliomas: a review of preclinical and correlative clinical data. (0360-3016 (Print)).

42. Weppler SA, Li Y Fau - Dubois L, Dubois L Fau - Lieuwes N, et al. Expression of EGFR variant vIll promotes both radiation resistance and hypoxia tolerance. (0167-8140 (Print)).

43. Draaisma K, Chatzipli A, Taphoorn M, et al. Molecular Evolution of IDH Wild-Type Glioblastomas Treated With Standard of Care Affects Survival and Design of Precision Medicine Trials: A Report From the EORTC 1542 Study. J Clin Oncol. 2020(1527-7755).

44. Emlet DR, Gupta P Fau - Holgado-Madruga M, Holgado-Madruga M Fau - Del Vecchio CA, et al. Targeting a glioblastoma cancer stem-cell population defined by EGF receptor variant III. Cancer Res. 2014;74(4):1238-1249.

45. Pelloski CE, Ballman KV, Furth AF, et al. Epidermal growth factor receptor variant III status defines clinically distinct subtypes of glioblastoma. J Clin Oncol. 2007;25(16):2288-2294.

46. Heimberger AB, Suki D, Yang D, Shi W, Aldape K. The natural history of EGFR and EGFRvIll in glioblastoma patients. J Transl Med. 2005;3:38.

47. Jutten B, Keulers TG, Schaaf MB, et al. EGFR overexpressing cells and tumors are dependent on autophagy for growth and survival. Radiother Oncol. 2013;108(3):479-483.

48. Rouschop KM, van den Beucken T, Dubois $L$, et al. The unfolded protein response protects human tumor cells during hypoxia through regulation of the autophagy genes MAP1LC3B and ATG5. J Clin Invest. 2010;120(1):127-141.

49. Sotelo J, Briceno E, Lopez-Gonzalez MA. Adding chloroquine to conventional treatment for glioblastoma multiforme: a randomized, double-blind, placebocontrolled trial. Ann Intern Med. 2006;144(5):337-343.

50. Jutten B, Keulers TG, Peeters HJM, et al. EGFRvIll expression triggers a metabolic dependency and therapeutic vulnerability sensitive to autophagy inhibition. Autophagy. 2018;14(2):283-295. 


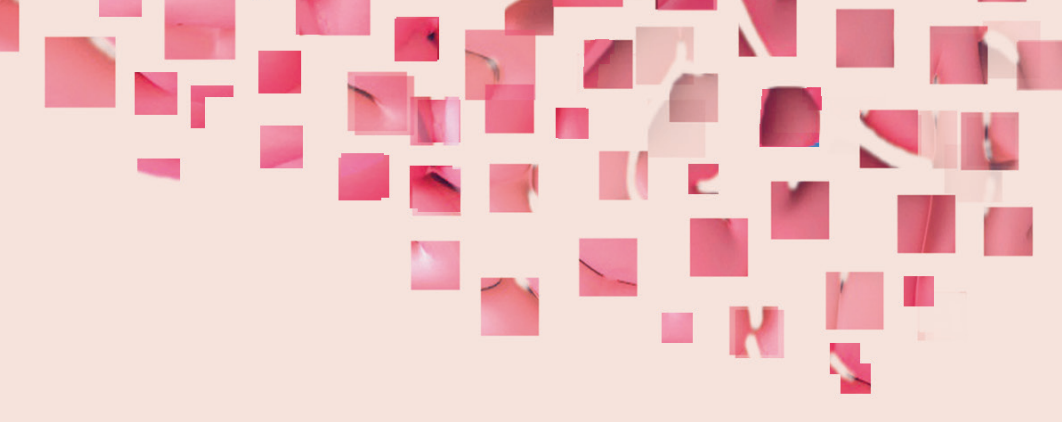

Part I

Decision support systems 


\section{Chapter 2 - Noninvasive Glioblastoma Testing: Multimodal Approach to Monitoring and Predicting Treatment Response}

Maikel Verduin, Inge Compter, Danny Steijvers, Alida A. Postma, Daniëlle B. P. Eekers, Monique M. Anten, Linda Ackermans, Mark ter Laan, Ralph T. H. Leijenaar, Tineke van de Weijer, Vivianne C. G. Tjan-Heijnen, Ann Hoeben, Marc Vooijs 


\section{Abstract}

Glioblastoma is the most aggressive adult primary brain tumor which is incurable despite intensive multimodal treatment. Inter- and intratumoral heterogeneity poses one of the biggest barriers in the diagnosis and treatment of glioblastoma, causing differences in treatment response and outcome. Non-invasive prognostic and predictive tests are highly needed to complement the current armamentarium. Non-invasive testing of glioblastoma uses multiple techniques that can capture the heterogeneity of glioblastoma. This set of diagnostic approaches comprises advanced MRI techniques, nuclear imaging, liquid biopsy and new integrated approaches including radiogenomics and radiomics. New treatment options such as agents targeted at driver oncogenes and immunotherapy are currently being developed, but benefit for glioblastoma patients still has to be demonstrated. Understanding and unraveling tumor heterogeneity and micro-environment can help to create a treatment regime that is patient-tailored to these specific tumor characteristics. Improved non-invasive tests are crucial to this success. This review discusses multiple diagnostic approaches and their effect on predicting and monitoring treatment response in glioblastoma. 


\section{Introduction}

Glioblastoma (GBM) is the most aggressive primary brain tumor with an incidence of 2-3 cases per 100.000 people ${ }^{1}$. Currently a median survival of approximately fourteen months is achieved with intensive multimodal treatment. However, despite this intensive treatment there is no cure and recurrence of GBM is inevitable ${ }^{2}$.

\section{Diagnosis and treatment}

Diagnostic approaches in GBM are rapidly evolving. The diagnosis is currently based on the recently revised WHO criteria (2016) for the classification of central nervous system tumors ${ }^{3}$. At present, histopathological investigation of a tissue sample from a suspected GBM lesion is the gold standard for the diagnosis. This is currently complemented by molecular diagnostics of which identification of O6-methylguanine-DNA-methyltransferase (MGMT) methylation, isocitrate dehydrogenase $(I D H)$-mutation and $1 \mathrm{p} 19 \mathrm{q}$ co-deletion are currently the most valuable in daily clinical practice. Methylation of the MGMT gene is the only predictive marker for treatment response available and is predictive of an improved response to alkylating chemotherapy such as temozolomide ${ }^{4,5}$. The IDH-mutation status $^{6}$ and 1 p19q co-deletion ${ }^{7}$ are of prognostic value but do not predict treatment response in patients with GBM. These markers are however of predictive value in oligodendroglioma $a^{8,9}$. Additional markers such as telomerase reverse transcriptase promotor (TERT) mutations and alpha-thalassemia syndrome X-linked (ATRX) can already be used additionally in the classification of GBM subtypes ${ }^{10,11}$.

At the moment, the treatment schedule consists of neurosurgery, concurrent chemoradiation therapy and adjuvant temozolomide. This provides a median progression free-survival of almost 7 months ${ }^{2}$. There is no standard treatment for the recurrent setting. Systemic treatment options include a temozolomide rechallenge, lomustine and anti-angiogenic therapy such as bevacizumab. However, effectiveness of these treatment options is limited. Additionally, re-irradiation and re-resection can be considered depending on the localization of the tumor and condition of the patient $^{12}$.

\section{Monitoring treatment response}

Both during and after treatment for GBM, magnetic resonance imaging (MRI) is the main modality used in the follow-up and monitoring of treatment response. Difficulties arise in monitoring response when it comes to the differentiation between pseudoprogression, radiation necrosis and actual tumor progression. Pseudoprogression is a physiologic post-treatment-related reaction of brain tissue, based on vascular and cellular injury from chemoradiation therapy. This leads to inflammation and increased permeability of the blood brain barrier (BBB), causing an increase in contrast enhancement on MRI suggestive of tumor progression but without tumor recurrence ${ }^{13,14}$. Radiation necrosis is a direct effect of radiation therapy, which can mimic tumor progression on imaging 
techniques but does not reflect actual progression of the tumor. Timing of MRI changes can help to differentiate between pseudoprogression, which occurs most commonly in the first three to six months post-treatment, and radiation necrosis which occurs six months to several years after treatment ${ }^{15}$.

Markers are also needed for monitoring treatment response for patients treated with immunotherapy. On MRI, these patients may first show an increase in size or even the formation of new (pseudo)lesions due to the anti-tumor mediated immune response and localized inflammation, which does not necessarily define progressive disease ${ }^{16,17}$. At the moment, differentiating pseudoprogression from actual tumor progression remains difficult and currently only follow-up imaging with conventional imaging methods is available to define this. Therefore, new imaging techniques and/ or biomarkers to further characterize the origin of the imaging changes that are observed are needed to overcome these challenges.

On the other hand, another phenomenon on MRI called pseudoresponse can also occur, mainly during treatment with vascular endothelial growth factor (VEGF) signaling pathway modifying agents such as bevacizumab. Bevacizumab induces a steroid-like effect by normalizing the permeability of the BBB, leading to a rapid decrease in contrast enhancement. Thus, while the imaging reflects reduced contrast and suggests a post-treatment response, the effects on overall survival are minimal $13,18,19$.

\section{Tumor heterogeneity}

Tumor heterogeneity poses one of the most important challenges in the current diagnosis and treatment of GBM and it is one of the main difficulties when it comes to finding new treatment options. GBM shows varying tumor characteristics both between patients as well as within individual tumors ${ }^{20,21}$. Current histological analysis cannot capture the full spectrum of genotypic and phenotypic characteristics, especially when only a single biopsy can be taken. The intratumoral heterogeneity poses a great challenge in predicting sensitivity and resistance to systemic therapies. As one clone within the tumor may be sensitive to one form of treatment, another clone might harbor certain resistance mechanisms to this treatment in its specific tumor micro-environment. Intratumoral heterogeneity is a dynamic process which changes over time and during treatment which poses challenges in the recurrent setting of GBM, as research has shown recurrent tumors usually show resistance to the traditional treatment options and expresses different mutations when compared to the original tumor ${ }^{20,22}$. Since conventional MRI cannot reflect tumor heterogeneity, before this can be used as a parameter in daily clinical practice, improved diagnostic approaches should be developed to identify this heterogeneity. 


\section{Non-invasive glioblastoma testing}

Non-invasive glioblastoma testing (NIGT) combines non-invasive (i.e. non-surgical) techniques to represent the tumor as a whole and provides information on driver mechanisms and tumor micro-environment, all of which are factors that can/should be incorporated into the treatment regime. This can be especially helpful in the selection of patients in order to better predict response to new therapeutic targets, as the current options available are limited. Also the brain is less easily accessible for taking repeated biopsies, which stresses the need for non-invasive approaches. New integrated approaches such as radiogenomics and radiomics can also be an important part of NIGT. Radiogenomics is an experimental diagnostic and predictive tool which studies the association between (qualitative) imaging features and molecular markers ${ }^{23}$. Radiomics on the other hand uses a computational analysis to extract quantifiable information about the underlying tumor characteristics by highthroughput mining of large amounts of quantitative features from images based on for example textures, intensities and shapes ${ }^{24,25}$. Radiomics has already been more extensively studied in for example head-and-neck cancer ${ }^{26,27}$ and lung cancer ${ }^{28}$.

The objective of this review is to discuss the already established approaches as well as future diagnostics used for monitoring and prediction of treatment response in patients with GBM by creating a so-called NIGT platform. This includes a multimodal approach to fully capture the complexity and heterogeneity of GBM with use of conventional techniques such as imaging techniques, enhanced by computational approaches and the use of circulating biomarkers.

\section{Magnetic Resonance Imaging}

MRI is clinically used in diagnosis and follow-up of cerebral tumors. The use of imaging to predict patient survival has been applied since as early as $1996^{29,30}$. Features found to be correlating with a longer survival in GBM are the presence of non-enhancing tumor and the absence of either edema, satellites or multifocality ${ }^{31}$. The Visually Accessible Rembrandt Images (VASARI) Research Project aimed to make MRI features more accurate and reproducible. In this project, a set of 24 observations describing the morphology of brain tumors on contrast-enhanced MRI were reported and analyzed for their prognostic significance on overall survival ${ }^{32,33}$.

\section{Monitoring treatment response}

For the evaluation of tumor response after first-line treatment, the Response Assessment in Neuro-Oncology (RANO) criteria of 2010 is currently used ${ }^{34}$. A major drawback of these criteria is its non-volumetric criteria and lack of use of advanced MR techniques. For instance, with using only these RANO criteria, pseudoprogression cannot be distinguished from radiation necrosis or disease recurrence ${ }^{35}$. 
Several advanced MR techniques have been developed to improve standard contrastenhanced MRI, such as diffusion weighted imaging (DWI), perfusion imaging and magnetic resonance spectroscopy (MRS) ${ }^{15}$. DWI displays the cellularity within tissue by detecting free diffusion of water molecules. The apparent diffusion coefficient (ADC) is a derived DWI parameter in which the T2 signal from the original DWI is excluded to overcome the so-called 'T2 shine-through effect', which causes a high signal on DWI that is not due to restricted diffusion. DWI and ADC are widely used in tumor imaging, where a decrease in ADC signal has been shown to correlate with increasing tumor cellularity while an increase in signal correlates with decreasing cellularity as a result of successful treatment ${ }^{36,37}$.

A relatively new DWI technique that has been developed is functional Diffusion Map imaging (fDM), which reflects differences in ADC signal over time. This fDM analysis has shown to be able to distinguish progressive tumors from stable and partially responsive tumors ${ }^{38,39}$. Although this technique is promising, there is a great variability in protocols collecting and processing DWI/ADC data between different vendors, standing in the way of wide scale use ${ }^{40}$.

Perfusion images can be acquired in various ways, with dynamic susceptibilityweighted contrast-enhanced MR (DSC MR) being most widely used. Other perfusion techniques include dynamic-contrast enhanced MR (DCE MR), which is comparable to DSC MR, and arterial spin labeling (ASL) perfusion, which does not require intravenous contrast but is more susceptible for artifacts. DSC MR is able to assess cerebral microvasculature by following an administered contrast agent as it passed through the microvasculature. Tumors tend to have a higher number and larger volumes of blood vessels. Furthermore, remodeling of the extracellular matrix disturbs the $\mathrm{BBB}$ and causes leakage of contrast ${ }^{14,41,42}$. By comparing e.g. tumor areas with healthy brain tissue, relative cerebral blood volumes ( $r C B V)$ can be measured ${ }^{14,41}$. The presence of high $\mathrm{rCBV}$ has been shown to represent active neovascularization and viable tumor, whereas normal rCBV in apparent lesion progression could point to e.g. chemoradiation effects and thereby exclude pseudoprogression and radionecrosis s $^{43,44}$.

MRS can be used to measure the distribution of chemical metabolites in brain tissue and thereby identifying differences in metabolic turn-over of brain tissue. As highgrade tumors are highly metabolically active and are accompanied by a leaky bloodbrain barrier, regional differences can be found in the spectroscopic profile in tumor depositions, compared to necrosis, pseudoprogression and healthy brain tissue. In $1 \mathrm{H}$-spectroscopy elevated peaks of lipid, lactate, choline, and myoinositol and reduced NAA signal are typical findings in primary brain tumors ${ }^{45,46}$. Due to patient and tumor specific differences an unequivocal threshold of metabolic signal ratios cannot be determined making it difficult to establish uniform guidelines and accuracy, however MRS changes in time can be of help to strengthen suspicions on for example tumor progression or response ${ }^{47}$. MRS alone therefore has a moderate diagnostic 
performance in differentiating glioma recurrence from radiation necrosis and should always be combined with other advanced imaging technologies ${ }^{48}$.

\section{Tumor heterogeneity and predicting treatment response}

GBM is subdivided into four subcategories based on histopathological features and specific mutations and molecular markers: proneural, neural, mesenchymal and classical subtypes. Each subset is associated with specific mutations; therefore, identification of the subtype by radiogenomics can provide information on driver mechanisms in the tumor. Between these subtypes, the proneural subtype is thought to have the most favorable prognosis ${ }^{49}$. Also different subtypes react differently to different treatment options $\mathrm{s}^{50}$. Radiogenomics can be applied to predict the GBM subtype. Volumes of both contrast enhancement and necrosis are higher in tumors with the mesenchymal subtype compared to the proneural subtype. GBMs with less than $5 \%$ tumor enhancement are mostly of the proneural subtype. On the other hand GBMs with less than 5\% non-enhanced tumor rarely represent proneural tumors and are more linked to the classical or mesenchymal subtype ${ }^{51,52}$.

Radiogenomics can also be used as tool to predict mutational status. IDH1mutational status is associated with a localization of the tumor in the frontal lobe, a higher percentage of non-contrast enhancing part of the tumor and the presence of cysts on MRI ${ }^{53,54}$. MRS has recently been used to predict IDH-mutation status. MRS can measure elevated levels of 2-HG metabolite which is a surrogate marker for IDH mutated tumor cells, and can correctly identify IDH mutation status in $88.6 \%$ of patients (sensitivity $89.5 \%$, specificity $81.3 \%$ ). However further technical improvement of this technique; including voxel localization, as well as understanding of the impact of tumor heterogeneity on MRS is needed before it can be used in daily clinical practice ${ }^{55,56}$.

MGMT-methylated tumors tend to be lateralized to the left temporal lobe whereas MGMT-unmethylated tumors are more frequent in the right hemisphere. This may be due to asymmetry in brain structure, function and gene expression between the hemispheres ${ }^{57}$. MGMT-unmethylated tumors have a higher percentage of tumor enhancement and T2/FLAIR hyperintensity when compared to MGMT-methylated tumors $^{53}$. Several imaging features are potential indicative of MGMT-methylation such as, mixed nodular enhancement, limited edema and moderately increased $\mathrm{rCBV}^{23}$.

The presence of the $1 p 19 q$ co-deletion is linked to classical oligodendroglial MRI characteristics such as heterogeneous T2 signal intensity and the presence of calcifications. Advanced imaging techniques have not yet shown to improve the capacity to identify the $1 \mathrm{p} 19 \mathrm{q}$ co-deletion over conventional MRI to identify oligodendroglial tumors ${ }^{23}$. 
Epidermal growth factor receptor (EGFR) amplification is associated with a significant higher percentage of contrast enhancement and T2/FLAIR hyperintensity compared with tumors lacking EGFR amplification. Also, EGFR-amplification and EGFRvIII mutant GBMs are commonly associated with localization in the left temporal lobe ${ }^{53}$. Apart from already mentioned molecular markers others known driver genes, such as phosphatase and tensin homolog (PTEN), platelet-derived growth factor receptor A (PDGFRA), cyclin-dependent kinase inhibitor 2A (CDKN2A), retinoblastoma 1 $(R B 1)$ and tumor protein ${ }^{53}$ (TP53) are also under investigation and significant image correlations for these genes have already been identified ${ }^{58}$.

The aforementioned advanced imaging techniques can also aid in exploring tumor heterogeneity. Both CBV and ADC measurements are found to be influenced by tumor aggressiveness and it is suggested that the heterogeneous genetic and cellular expression patterns within GBM influence anatomic and physiologic MR imaging ${ }^{59}$. These techniques can also guide neurosurgeons in determining the biopsy location.

MRI-based radiomics for GBM is a relatively new area for which little research has been published to this date. A study of 82 GBM patients reported favorable results in the performance of texture features in predicting the molecular subtype and 12 -month survival ${ }^{60}$. For the prediction of 12 -month survival based on pattern analysis sensitivity and specificity of 0.86 and 0.64 are reported. The prediction of GBM subtype was also investigated. Accuracy for classical, mesenchymal, neural and proneural subtypes were $0.88,0.70,0.85$ and 0.93 respectively ${ }^{61}$. Another study used machine-learning techniques and found an accuracy of almost $80 \%$ in predicting overall survival and an accuracy of $76 \%$ in predicting the molecular subtype ${ }^{62}$.

MRI texture analysis has been found to be able to facilitate in characterizing intratumoral heterogeneity and may therefore aid in identifying genetically different components of the tumor and understanding its consequences for prognosis, treatment sensitivity and resistance ${ }^{58}$. It has been shown that radiomics features are able to visualize spatial gene-expression within a tumor ${ }^{63}$. Patients can be subdivided into different clusters using texture features. For example, one study divided patients into the 'pre-multifocal', 'spherical' or 'rim-enhancing' cluster based on quantitative imaging features. Each of these clusters was linked to different signaling pathways and microarrays and has been shown to be prognostic for survival ${ }^{64}$.

Radiomics was found to have prognostic value for both survival and progression in patients with recurrent GBM receiving bevacizumab. Therefore it might be possible to develop pre-treatment biomarkers based on radiomics to predict benefit from bevacizumab ${ }^{65,66}$. These findings illustrate the possibilities of applying radiomics in the prediction of treatment response in patients with GBM. Further optimization of this technique and validation of radiomics profiles as predictors for different mutation statuses and/or survival is needed before it can be used in clinical practice. 


\section{Nuclear imaging}

Molecular imaging by the use of positron emission tomography (PET) is increasingly being implemented into clinical practice for treatment planning and response monitoring of GBM. The most common is fluorodeoxyglucose $\left({ }^{18} \mathrm{~F}-\mathrm{FDG}\right)$ PET imaging; however, compared to other organ systems, ${ }^{18} \mathrm{~F}$-FDG-PET imaging of brain tumors presents unique challenges because of the high background glucose metabolism of normal gray matter masking detection of malignant lesions. Thus, the use of ${ }^{18} \mathrm{~F}-\mathrm{FDG}-$ PET in brain tumors albeit is limited, although in high grade glioma, ${ }^{18} \mathrm{~F}-\mathrm{FDG}-\mathrm{PET}$ imaging can be used to identify metabolically active disease which correlates with tumor grade ${ }^{67,68}$. Because of the limited utility of ${ }^{18} \mathrm{~F}-\mathrm{FDG}$-PET, the RANO working group has recommended the use of radio-labeled amino acid tracers for PET (AAPET) instead ${ }^{69}$.

Amino acid tracers such as O-(2-[18 F]-fluoroethyl)-L-tyrosine $\left({ }^{18} \mathrm{~F}-\mathrm{FET}\right)$ and L-[Methyl$\left.{ }^{11} \mathrm{C}\right]$ methionine $\left({ }^{11} \mathrm{C}-\mathrm{MET}\right)$ are currently applied in clinical practice in GBM. Both ${ }^{18} \mathrm{~F}$-FET and ${ }^{11} \mathrm{C}-\mathrm{MET}$ show rapid uptake into tumors and can be visualized with high contrast ${ }^{70} .{ }^{11} \mathrm{C}$ has a short half life time of 20 minutes, making it less useful for clinical practice. ${ }^{18} \mathrm{~F}$ has a much longer half life time (120 minutes) and is only taken up by cells through specific L-transporters -LAT2- that are highly and predominantly expressed on glioma tumor cells. This ensures a high and selective uptake of the tracer in tumor tissue, with a low to negligible background signal in normal brain tissue or in surrounding inflammatory areas. Thus, ${ }^{18} \mathrm{~F}-\mathrm{FET}$-PET has a high sensitivity and specificity for the detection of malignant gliomas ${ }^{71-74}$. A biopsy-controlled study has shown that with a combination of MRI and $18 \mathrm{~F}-\mathrm{FET}$-PET a sensitivity of $93 \%$ and a specificity of $94 \%$ can be achieved ${ }^{71}$. Other amino acid tracers currently under investigation include ${ }^{18} \mathrm{~F}-\mathrm{FDOPA}$ (phenyl alanine) PET, a dopaminergic tracer, and alpha ${ }^{11} \mathrm{C}$-L-methyl-tryptophan PET, a tryptophan analog.

\section{Monitoring treatment response}

${ }^{18}$ F-FET-PET currently has multiple potential clinical applications including the monitoring of treatment response and can distinguish tumor recurrence from radiation necrosis or pseudoprogression ${ }^{69}$. A study investigating ${ }^{18} \mathrm{~F}-\mathrm{FET}$ for PETguided radiotherapy concluded that size and geometrical location of gross tumor volume and biological tumor volume, defined by ${ }^{18} \mathrm{~F}-\mathrm{FET}$ uptake, were significantly different in patients where the biological tumor volume extended up to $10-20 \mathrm{~mm}$ from the margin of contrast enhancement on MRI, potentially improving local tumor control due to improved radiotherapy planning using ${ }^{18} \mathrm{~F}-\mathrm{FET}-\mathrm{PET}^{75}$. Multiple studies have shown that both ${ }^{18} \mathrm{~F}-\mathrm{FET}$-PET and ${ }^{18} \mathrm{~F}$-FDOPA-PET have a higher diagnostic accuracy than conventional MRI in differentiating glioma recurrence from posttreatment tissue changes. For example, studies show a sensitivity and specificity of $92.3 \%$ and $44.4 \%$ respectively for MRI compared to $100 \%$ and $88.89 \%{ }^{18} \mathrm{~F}-\mathrm{FDOPA}$ $\mathrm{PET}^{76-79}$. A prospective study investigating the predictive value of ${ }^{18} \mathrm{~F}-\mathrm{FET}-\mathrm{PET}$ in 
patients treated with chemoradiation has shown that a decrease of ${ }^{18} \mathrm{~F}$-FET-PET accumulation reflects tumor response to the therapeutic intervention at an early stage of the disease and predicts outcome, whereas contrast-enhanced MRI did not ${ }^{78}$.

\section{Tumor heterogeneity and predicting treatment response}

Correlation between different types of AA-PET standard uptake values (SUV) and molecular markers, in the context of radiogenomics, is currently under investigation. A longitudinal prospective study has investigated 18F-FET-PET as an imaging biomarker and they concluded that the biological tumor volume before treatment was a strong prognostic marker for both overall- and progression-free survival independent of treatment as well as MGMT promoter methylation, and other patient-and tumor-related factors. Moreover, tumor uptake kinetics before and after treatment (i.e. TAC curves) are correlated with progression-free survival ${ }^{80}$. A recent study demonstrated the relationship between ${ }^{11} \mathrm{C}-\mathrm{MET}$-PET and IDH1mutation, and found that SUVmax and SUVratio were inversely correlated with IDH1 mutation ${ }^{81}$. Moreover, a study which combined MRI and alpha[C-11]-L-methyltryptophan PET imaging showed prognostic imaging factors such as T1-contrast/PET volume ratios and metabolic volume, which are associated with EGFR amplification and MGMT-methylation status ${ }^{82}$. To assess the potential of radiogenomics as a diagnosis and predictive tool, well defined preclinical models with specific driver mutations are needed that can be used to validate the sensitivity and specificity of radiogenomics. The ability to identify prognostic or molecular response markers based on imaging features derived from routine diagnostic procedures (MRI, PET, computed tomography $(\mathrm{CT})$ ) provides an attractive way of predicting treatment response in GBM.

Tumor hypoxia is a common feature of the tumor microenvironment in GBM and contributes to increased malignancy, poor prognosis and resistance to radiotherapy and alkylating chemotherapy such a temozolomide 83-85. Acute and chronic hypoxic areas fluctuate in human tumors and contribute to spatial and temporal intratumoral heterogeneity ${ }^{86,87}$. This has a significant impact on resistance to conventional treatment. Therefore GBM patients might benefit from hypoxia targeting drugs ${ }^{21}$. Molecular imaging of tumor hypoxia could aid in the selection of patients with hypoxic tumors, which could benefit from specific anti-hypoxic therapies. The efficacy of anti-hypoxic treatments will depend on the presence of hypoxia. Several 2-nitroimidazoles, labeled with $18 \mathrm{~F}$ have already been investigated in patients to identify hypoxia ${ }^{88}$. In extensive pre-clinical models and clinical trials ${ }^{8} \mathrm{~F}-\mathrm{HX} 4-\mathrm{PET}$ has shown to be a promising and non-toxic probe for hypoxia ${ }^{89-91}$. Repeated hypoxiaimaging during the course of disease and treatment will demonstrate the extent of spatial and temporal fluctuations in tumor hypoxia and is likely important in scheduling hypoxia-modifying drug in combination with conventional treatments. 


\section{Liquid biopsy}

Liquid biopsy (LB) has entered clinical practice in the treatment of several cancer types, including breast and colorectal cancer ${ }^{92,93}$. LB studies circulating biomarkers which refer to measurable biological molecules found in blood, urine and or other body fluids, like cerebrospinal fluid (CSF). Although LB has refined the individual treatment for several cancer subtypes, relatively little progress has been made with regards to validation of circulating biomarkers for primary brain tumors. Nevertheless, although the translation of biomarker development into neuro-oncology is lagging behind compared to general oncology, the prerequisites for adequate extrapolation are present, mostly in experimental studies ${ }^{94}$. In these studies, circulating tumor cells (CTCs), circulating free nuclear acids (cfNA), extracellular vesicles and circulating proteins and metabolites have been described.

For brain tumors, where non-invasive procedures are complex, precarious and may be non-representative for outcome, circulating biomarkers pose a realistic option. For the inoperable patients, which mostly occurs in the recurrent setting, circulating biomarkers could be the source of a molecular profile of the relapsed tumor, allowing clinicians to identify potentially druggable molecular alterations driving recurrence.

\section{Monitoring treatment response}

miRNAs are small (about 21-24 nucleotides) non-coding regulatory RNA molecules and can be detected as cell free entities or as the content of circulating extracellular vesicles (EVs) in plasma/serum or CSF. EVs are small nanometer size membrane-enclosed particles that are released from GBM living tumor cells. EVs that can be isolated from both blood and CSF are a rich source of tumorderived molecules such as DNA, miRNA, mRNA, proteins, lipids and metabolites, because the structure of EVs protects them from nucleases and proteases ${ }^{95}$. The human genome encodes for miRNAs which have been shown to regulate most hallmarks of tumor development and progression via transcriptional silencing or translation inhibition of both oncogene and suppressor genes and have tumor/ tissue specific signatures ${ }^{96}$. miRNAs have been described in GBM, mostly from resected specimens. miR-21 is the most reliable plasma biomarker in glioma diagnosis and seems to be valuable distinguishing tumor progression from pseudoprogression or radionecrosis ${ }^{97}$. Exosomal miRNA-21 in CSF of glioma patients has been shown to correlate with glioma recurrence ${ }^{98}$. An increase in levels of blood born annexin $\mathrm{V}$ positive microvesicles during chemoradiation is associated with earlier recurrence and shorter overall survival. Since the number of patients included in this analysis was only small $(n=16)$ further investigation is needed ${ }^{99}$.

\section{Tumor heterogeneity and predicting treatment response}

The ability to detect CTCs in GBM patients has been established ${ }^{100}$. Also, using single cell genome sequencing, some unique mutations were found in the CTCs as well as 
in the parental tumor ${ }^{101}$. However, the most important limiting factor for the clinical implementation of CTCs is their scarcity, which makes it difficult to adequately assess GBM heterogeneity. Also it has not yet been established in GBM if CTCs can be identified which show characteristics of brain tumor initiating cells.

Circulating tumor DNA (ctDNA) is much more abundant than CTCs and contains the mutations present in tumors ${ }^{102}$. In GBM patients a lower rate of ctDNA is detected compared to other solid tumors, mainly due to the only partially disrupted BBB. In several small retrospective studies ctDNAs were successfully detected in GBM patients and multiple molecular alterations were characterized including loss of heterozygosity (LOH) in chromosomes arms $1 p, 19 q$ and 10q, IDH1 and EGFRvIII mutations as well as methylation of promoters of MGMT, PTEN and CDKN2A ${ }^{103-105}$. Only few studies have reported on the in plasma half-life of ctDNA. The available data propose that the fast turnover of ctDNA reflects tumor homeostasis ${ }^{106}$. Until now, the clinical utility of candidate ctDNAs as biomarkers for patients with GBM has not been demonstrated and large scale prospective studies are needed before their implementation in clinical practice.

miR-130a was found to positively correlate with temozolomide response in GBM patients, independent from MGMT methylation status ${ }^{107}$ miR-603 is another regulator of $M G M T$ and could complement assessment of $M G M T$ methylation, which alone cannot completely explain temozolomide efficiency, as a predictive marker for treatment response ${ }^{108}$. miR-181 d levels in the serum of GBM patients is also shown to correlate with response to temozolomide; since this miRNA, like miR-603, is directly involved in the downregulation of MGMT109.

Mutant IDH enzymes acquire neomorphic enzymatic activity, thereby catalyzing the production of D-2-hydroxyglutarate (D2HG), an oncometabolite that accumulates at high levels and inhibits several enzymes notably involved in histones and DNA demethylation ${ }^{110}$. In most patients with $I D H 1 / 2$ mutant gliomas, plasma D2HG values are in the normal range ${ }^{111}$, suggesting limited clinical value of this oncometabolite. However, combining this technique with MRS which can, as mentioned before, measure D2HG metabolite concentrations in brain tissue, might be useful in exploring IDH mutation status.

Several circulating proteins have been evaluated and include proteins with cell lineage such as GFAP $P^{112}, N C A M^{113}$ and $S 100 B^{114}$, matricellular proteins and matrix metalloproteinases such as $Y K L-40, M M P 2, M M P 91^{15}$, TIMP-1 and osteopontin ${ }^{116}$ and cytokines ${ }^{117}$, growth factors and growth factor receptors such as VEGF, FGF-2, PIGF, IGFBP-5, EGFR, VEGFR $1^{118}$ and TGF- $\beta 1^{119}$. Further validation of these biomarkers is warranted. 


\section{Discussion and future perspectives}

Advanced MR imaging techniques, nuclear imaging, liquid biopsy and integrated radiogenomics and radiomics approaches are examples of non-invasive diagnostic methods to uncover underlying tumor characteristics. GBM is a challenging tumor both from a diagnostic and therapeutic point of view. Recent advances are made when it comes to molecular markers and the understanding of underlying driver oncogenes and tumor micro-environment, all factors which contribute to treatment sensitivity and resistance. Diagnostic methods to accurately identify these factors and their impact on outcome are needed to be able to put this knowledge to clinical use.

Tumor heterogeneity poses a big challenge in the use of targeted treatment approaches. Apart from heterogeneity on the genetic level, non-genetic factors such as the tumor micro-environment also influence the development on cancer cell populations ${ }^{120}$. Different niches have been identified in GBM harboring very different epigenetic and environmental factors which also play a role in treatment resistance and heterogeneity ${ }^{121}$. These differences make it challenging to create one uniform treatment schedule for GBM and a comprehensive insight into the behavior of these distinct tumor cell populations is needed.

The diagnostic modalities previously discussed all have future possibilities to improve the understanding of tumor heterogeneity and the prediction of treatment response as well as the monitoring of treatment response with regards to the differentiation of pseudoprogression, radiation necrosis and actual tumor progression. The latter is especially important when it comes to patients treated with immunotherapy, for which no adequate distinction between pseudoprogression and actual progression can currently be made.

MRI is already well established within the clinic of GBM, further optimization through higher resolutions (e.g. ultra-high field MRI), wider use of advanced imaging techniques and further research, including clinical validation, on the application of radiogenomics will improve the diagnostic power of MRI. The same applies to nuclear imaging, especially different types of AA-PET for which additional studies on known amino acid tracers and the development of new tracers to improve diagnostic accuracy, both in the setting of the primary diagnosis as well as in the monitoring of treatment response and patient follow-up, is warranted. Radiomics is an important topic in different types of solid tumors and still relatively new in GBM. Radiomics analysis is one of the most promising techniques for the differentiation between different GBM subsets and in evaluating and monitoring intratumoral heterogeneity ${ }^{122}$. Ideally predictive radiomics models are created as predictors for GBM subtypes as has already been established using radiomics in other types of solid tumors ${ }^{24}$. Before radiomics can be applied prospective 
validation is needed as well as standardization of imaging protocols, imaging segmentation and feature extraction to ensure interoperability of multi-center radiomics studies ${ }^{123}$.

Liquid biopsy provides a different approach for understanding tumor characteristics. Further research should focus on determining the clinical value of liquid biopsy and also which liquid source and which biomarker technique to use. Also, the possibility to use liquid biopsy in patients after a tumor resection as a marker for tumor recurrence has yet to be studied. Tumor heterogeneity will remain an important pitfall in liquid biopsy technique; this might be overcome by combining liquid biopsy with other non-invasive markers but will remain a challenge.

Future research should focus on determining the sensitivity and specificity and validating the techniques previously discussed for GBM. Being able to understand and unravel intratumoral heterogeneity provides clinicians with important information to create the most optimal treatment regime and this should therefore be the focus of future studies.

NIGT offers a non-invasive panel to understand the driver mechanisms as potential treatment targets as well as identifying the tumor micro-environment. Combining different diagnostic modalities aims to achieve optimal diagnostic power for identification of tumor characteristics. Understanding the tumor micro-environment (e.g. hypoxia, angiogenesis and immune infiltration) can help in finding new ways to treat GBM or to alter the tumor micro-environment to improve the effectiveness of systemic therapies and radiotherapy. Unique to the brain micro-environment is the BBB which limits effectiveness of therapeutic agents. Advances in imaging allow the visualization of changes in the tumor micro-environment and tissue architecture as a response to treatment and can therefore serve as a marker for treatment response.

Given the limitations of each of the currently available non-invasive tools these diagnostic methods are ideally combined into a so-called NIGT platform: a multimodal non-invasive approach to visualize the tumor and its underlying tumor characteristics in a spatially and temporally relevant manner. Using this NIGT platform, predictive models for GBM can be created, both in the primary and the recurrent setting. This will guide clinicians in selecting the appropriate treatment option treatment monitoring and adaptation in the era of patient-tailored precision medicine. 
1. Urbanska K, Sokolowska J, Szmidt M, Sysa P. Glioblastoma multiforme - an overview. Contemp Oncol (Pozn) 2014; 18: 307-312.

2. Stupp R, Mason WP, van den Bent MJ et al. Radiotherapy plus concomitant and adjuvant temozolomide for glioblastoma. N Engl J Med 2005; 352: 987-996.

3. Louis DN, Perry A, Reifenberger G et al. The 2016 World Health Organization Classification of Tumors of the Central Nervous System: a summary. Acta Neuropathol 2016; 131: 803-820.

4. Esteller M, Garcia-Foncillas J, Andion E et al. Inactivation of the DNA-repair gene MGMT and the clinical response of gliomas to alkylating agents. N Engl J Med 2000; 343: 1350-1354.

5. Hegi ME, Diserens AC, Gorlia T et al. MGMT gene silencing and benefit from temozolomide in glioblastoma. N Engl J Med 2005; 352: 997-1003.

6. Dahlrot RH, Kristensen BW, Hjelmborg J et al. A population-based study of highgrade gliomas and mutated isocitrate dehydrogenase 1. Int J Clin Exp Pathol 2013; 6: 31-40.

7. Aldape K, Burger PC, Perry A. Clinicopathologic aspects of $1 p / 19 q$ loss and the diagnosis of oligodendroglioma. Arch Pathol Lab Med 2007; 131: 242-251.

8. Cairncross G, Wang M, Shaw E et al. Phase III trial of chemoradiotherapy for anaplastic oligodendroglioma: long-term results of RTOG 9402. J Clin Oncol 2013; 31: 337-343.

9. Cairncross JG, Wang M, Jenkins RB et al. Benefit from procarbazine, lomustine, and vincristine in oligodendroglial tumors is associated with mutation of IDH. J Clin Oncol 2014; 32: 783-790.

10. Eckel-Passow JE, Lachance DH, Molinaro AM et al. Glioma Groups Based on 1p/19q, IDH, and TERT Promoter Mutations in Tumors. N Engl J Med 2015; 372: 2499-2508.

11. van den Bent MJ, Weller M, Wen PY et al. A clinical perspective on the 2016 WHO brain tumor classification and routine molecular diagnostics. Neuro Oncol 2017; 19: 614-624.

12. van Linde ME, Brahm CG, de Witt Hamer PC et al. Treatment outcome of patients with recurrent glioblastoma multiforme: a retrospective multicenter analysis. J Neurooncol 2017.

13. Brandsma D, van den Bent MJ. Pseudoprogression and pseudoresponse in the treatment of gliomas. Curr Opin Neurol 2009; 22: 633-638.

14. Korfiatis $P$, Erickson $B$. The basics of diffusion and perfusion imaging in brain tumors. Appl Radiol 2014; 43: 22-29. 
15. Ellingson BM, Chung C, Pope WB et al. Pseudoprogression, radionecrosis, inflammation or true tumor progression? challenges associated with glioblastoma response assessment in an evolving therapeutic landscape. J Neurooncol 2017.

16. Huang RY, Neagu MR, Reardon DA, Wen PY. Pitfalls in the neuroimaging of glioblastoma in the era of antiangiogenic and immuno/targeted therapy detecting illusive disease, defining response. Front Neurol 2015; 6: 33.

17. Okada $H$, Weller $M$, Huang $R$ et al. Immunotherapy response assessment in neurooncology: a report of the RANO working group. Lancet Oncol 2015; 16: e534-542.

18. Chang JH, Kim CY, Choi BS et al. Pseudoprogression and pseudoresponse in the management of high-grade glioma : optimal decision timing according to the response assessment of the neuro-oncology working group. J Korean Neurosurg Soc 2014; 55: 5-11.

19. Batchelor TT, Sorensen AG, di Tomaso E et al. AZD2171, a pan-VEGF receptor tyrosine kinase inhibitor, normalizes tumor vasculature and alleviates edema in glioblastoma patients. Cancer Cell 2007; 11: 83-95.

20. Qazi MA, Vora P, Venugopal C et al. Intratumoral Heterogeneity: Pathways to Treatment Resistance and Relapse in Human Glioblastoma. Ann Oncol 2017.

21. Vartanian A, Singh SK, Agnihotri S etal.GBM's multifaceted landscape: highlighting regional and microenvironmental heterogeneity. Neuro Oncol 2014; 16: 11671175.

22. Johnson $\mathrm{BE}$, Mazor $\mathrm{T}$, Hong $\mathrm{C}$ et al. Mutational analysis reveals the origin and therapy-driven evolution of recurrent glioma. Science 2014; 343: 189-193.

23. Smits $M$, van den Bent MJ. Imaging Correlates of Adult Glioma Genotypes. Radiology 2017; 284: 316-331.

24. Aerts HJ, Velazquez ER, Leijenaar RT et al. Decoding tumour phenotype by noninvasive imaging using a quantitative radiomics approach. Nat Commun 2014; 5: 4006.

25. Lambin P, Rios-Velazquez E, Leijenaar $\mathrm{R}$ et al. Radiomics: extracting more information from medical images using advanced feature analysis. Eur $\mathrm{J}$ Cancer 2012; 48: 441-446.

26. Leijenaar RT, Carvalho S, Hoebers FJ et al. External validation of a prognostic CT-based radiomic signature in oropharyngeal squamous cell carcinoma. Acta Oncol 2015; 54: 1423-1429.

27. Parmar C, Grossmann P, Rietveld D et al. Radiomic Machine-Learning Classifiers for Prognostic Biomarkers of Head and Neck Cancer. Front Oncol 2015; 5: 272.

28. Wu W, Parmar C, Grossmann P et al. Exploratory Study to Identify Radiomics Classifiers for Lung Cancer Histology. Front Oncol 2016; 6: 71. 
29. Hammoud MA, Sawaya R, Shi W et al. Prognostic significance of preoperative MRI scans in glioblastoma multiforme. J Neurooncol 1996; 27: 65-73.

30. Pierallini A, Bonamini $M$, Pantano $P$ et al. Radiological assessment of necrosis in glioblastoma: variability and prognostic value. Neuroradiology 1998; 40: 150-153.

31. Pope WB, Sayre J, Perlina A et al. MR imaging correlates of survival in patients with high-grade gliomas. AJNR Am J Neuroradiol 2005; 26: 2466-2474.

32. Wangaryattawanich $P$, Hatami $M$, Wang $J$ et al. Multicenter imaging outcomes study of The Cancer Genome Atlas glioblastoma patient cohort: imaging predictors of overall and progression-free survival. Neuro Oncol 2015; 17: 15251537.

33. The Cancer Imaging Archive. Wiki for the VASARI feature set. In. 2013.

34. Wen PY, Macdonald DR, Reardon DA et al. Updated response assessment criteria for high-grade gliomas: response assessment in neuro-oncology working group. J Clin Oncol 2010; 28: 1963-1972.

35. Wen PY, Chang SM, Van den Bent MJ et al. Response Assessment in NeuroOncology Clinical Trials. J Clin Oncol 2017; 35: 2439-2449.

36. Chenevert TL, McKeever PE, Ross BD. Monitoring early response of experimental brain tumors to therapy using diffusion magnetic resonance imaging. Clin Cancer Res 1997; 3: 1457-1466.

37. Sugahara T, Korogi $Y$, Kochi $M$ et al. Usefulness of diffusion-weighted MRI with echo-planar technique in the evaluation of cellularity in gliomas. J Magn Reson Imaging 1999; 9: 53-60.

38. Moffat BA, ChenevertTL, LawrenceTS et al. Functional diffusion map: a noninvasive MRI biomarker for early stratification of clinical brain tumor response. Proc Natl Acad Sci U S A 2005; 102: 5524-5529.

39. Moffat BA, Chenevert TL, Meyer CR et al. The functional diffusion map: an imaging biomarker for the early prediction of cancer treatment outcome. Neoplasia 2006; 8: 259-267.

40. Schmainda KM. Diffusion-weighted MRI as a biomarker for treatment response in glioma. CNS Oncol 2012; 1: 169-180.

41. Gahramanov S, Raslan AM, Muldoon LL et al. Potential for differentiation of pseudoprogression from true tumor progression with dynamic susceptibilityweighted contrast-enhanced magnetic resonance imaging using ferumoxytol vs. gadoteridol: a pilot study. Int J Radiat Oncol Biol Phys 2011; 79: 514-523.

42. Nasseri M, Gahramanov S, Netto JP et al. Evaluation of pseudoprogression in patients with glioblastoma multiforme using dynamic magnetic resonance imaging with ferumoxytol calls RANO criteria into question. Neuro Oncol 2014; 16: 1146-1154. 
43. Law M, Young RJ, Babb JS et al. Gliomas: predicting time to progression or survival with cerebral blood volume measurements at dynamic susceptibility-weighted contrast-enhanced perfusion MR imaging. Radiology 2008; 247: 490-498.

44. Hu LS, Eschbacher JM, Heiserman JE et al. Reevaluating the imaging definition of tumor progression: perfusion MRI quantifies recurrent glioblastoma tumor fraction, pseudoprogression, and radiation necrosis to predict survival. Neuro Oncol 2012; 14: 919-930.

45. Al-Okaili RN, Krejza J, Wang S et al. Advanced MR imaging techniques in the diagnosis of intraaxial brain tumors in adults. Radiographics 2006; 26 Suppl 1: S173-189.

46. Wang $\mathrm{Q}$, Zhang $\mathrm{H}$, Zhang J et al. The diagnostic performance of magnetic resonance spectroscopy in differentiating high-from low-grade gliomas: $A$ systematic review and meta-analysis. Eur Radiol 2016; 26: 2670-2684.

47. Choi C, Raisanen JM, Ganji SK et al. Prospective Longitudinal Analysis of 2-Hydroxyglutarate Magnetic Resonance Spectroscopy Identifies Broad Clinical Utility for the Management of Patients With IDH-Mutant Glioma. J Clin Oncol 2016; 34: 4030-4039.

48. Zhang H, Ma L, Wang $\mathrm{Q}$ et al. Role of magnetic resonance spectroscopy for the differentiation of recurrent glioma from radiation necrosis: a systematic review and meta-analysis. Eur J Radiol 2014; 83: 2181-2189.

49. Verhaak RG, Hoadley KA, Purdom E et al. Integrated genomic analysis identifies clinically relevant subtypes of glioblastoma characterized by abnormalities in PDGFRA, IDH1, EGFR, and NF1. Cancer Cell 2010; 17: 98-110.

50. Prins RM, Soto $H$, Konkankit $V$ et al. Gene expression profile correlates with T-cell infiltration and relative survival in glioblastoma patients vaccinated with dendritic cell immunotherapy. Clin Cancer Res 2011; 17: 1603-1615.

51. Naeini KM, Pope WB, Cloughesy TF et al. Identifying the mesenchymal molecular subtype of glioblastoma using quantitative volumetric analysis of anatomic magnetic resonance images. Neuro Oncol 2013; 15: 626-634

52. Gutman DA, Cooper LA, Hwang SN et al. MR imaging predictors of molecular profile and survival: multi-institutional study of the TCGA glioblastoma data set. Radiology 2013; 267: 560-569.

53. Ellingson BM, Lai A, Harris RJ et al. Probabilistic radiographic atlas of glioblastoma phenotypes. AJNR Am J Neuroradiol 2013; 34: 533-540.

54. Carrillo JA, Lai A, Nghiemphu PL et al. Relationship between tumor enhancement, edema, IDH1 mutational status, MGMT promoter methylation, and survival in glioblastoma. AJNR Am J Neuroradiol 2012; 33: 1349-1355. 
55. Tietze A,ChoiC, MickeyBetal.Noninvasiveassessment of isocitrate dehydrogenase mutation status in cerebral gliomas by magnetic resonance spectroscopy in a clinical setting. J Neurosurg 2017; 1-8.

56. Leather T, Jenkinson MD, Das K, Poptani H. Magnetic Resonance Spectroscopy for Detection of 2-Hydroxyglutarate as a Biomarker for IDH Mutation in Gliomas. Metabolites 2017; 7.

57. Ellingson BM, Cloughesy TF, Pope WB et al. Anatomic localization of O6methylguanine DNA methyltransferase (MGMT) promoter methylated and unmethylated tumors: a radiographic study in 358 de novo human glioblastomas. Neuroimage 2012; 59: 908-916.

58. Hu LS, Ning S, Eschbacher JM et al. Radiogenomics to characterize regional genetic heterogeneity in glioblastoma. Neuro Oncol 2017; 19: 128-137.

59. Barajas RF, Hodgson JG, Chang JS et al. Glioblastoma Multiforme Regional Genetic and Cellular Expression Patterns: Influence on Anatomic and Physiologic MR Imaging. Radiology 2010; 254: 564-576.

60. Yang D, Rao G, Martinez J et al. Evaluation of tumor-derived MRI-texture features for discrimination of molecular subtypes and prediction of 12-month survival status in glioblastoma. Med Phys 2015; 42: 6725-6735.

61. Lee J, Narang S, Martinez J et al. Spatial Habitat Features Derived from Multiparametric Magnetic Resonance Imaging Data Are Associated with Molecular Subtype and 12-Month Survival Status in Glioblastoma Multiforme. PLoS One 2015; 10: e0136557.

62. Macyszyn L, Akbari H, Pisapia JM et al. Imaging patterns predict patient survival and molecular subtype in glioblastoma via machine learning techniques. Neuro Oncol 2016; 18: 417-425.

63. Diehn M, Nardini C, Wang DS et al. Identification of noninvasive imaging surrogates for brain tumor gene-expression modules. Proc Natl Acad Sci U S A 2008; 105 : 5213-5218.

64. Itakura H, Achrol AS, Mitchell LA et al. Magnetic resonance image features identify glioblastoma phenotypic subtypes with distinct molecular pathway activities. Sci Transl Med 2015; 7: 303ra138.

65. Kickingereder P, Gotz M, Muschelli J et al. Large-scale Radiomic Profiling of Recurrent Glioblastoma Identifies an Imaging Predictor for Stratifying AntiAngiogenic Treatment Response. Clin Cancer Res 2016; 22: 5765-5771.

66. Grossmann P, Narayan V, Chang K et al. Quantitative Imaging Biomarkers for Risk Stratification of Patients with Recurrent Glioblastoma Treated with Bevacizumab. Neuro Oncol 2017. 
67. Delbeke D, Meyerowitz C, Lapidus RL et al. Optimal cutoff levels of F-18 fluorodeoxyglucose uptake in the differentiation of low-grade from high-grade brain tumors with PET. Radiology 1995; 195: 47-52.

68. Goldman S, Levivier M, Pirotte B et al. Regional glucose metabolism and histopathology of gliomas. A study based on positron emission tomographyguided stereotactic biopsy. Cancer 1996; 78: 1098-1106.

69. Albert NL, Weller M, Suchorska B et al. Response Assessment in Neuro-Oncology working group and European Association for Neuro-Oncology recommendations for the clinical use of PET imaging in gliomas. Neuro Oncol 2016; 18: 1199-1208.

70. Grosu AL, Astner ST, Riedel E et al. An interindividual comparison of O-(2-[18F] fluoroethyl)-L-tyrosine (FET)- and L-[methyl-11C]methionine (MET)-PET in patients with brain gliomas and metastases. Int J Radiat Oncol Biol Phys 2011; 81: 1049-1058.

71. Pauleit D, Floeth F, Hamacher $\mathrm{K}$ et al. O-(2-[18F]fluoroethyl)-L-tyrosine PET combined with MRI improves the diagnostic assessment of cerebral gliomas. Brain 2005; 128: 678-687.

72. Kracht LW, Miletic H, Busch S et al. Delineation of brain tumor extent with [11C] L-methionine positron emission tomography: local comparison with stereotactic histopathology. Clin Cancer Res 2004; 10: 7163-7170.

73. Pirotte B, Goldman S, Massager N et al. Comparison of 18F-FDG and $11 \mathrm{C}-$ methionine for PET-guided stereotactic brain biopsy of gliomas. J Nucl Med 2004; 45: 1293-1298.

74. Rachinger W, Goetz C, Popperl G et al. Positron emission tomography with O-(2[18F]fluoroethyl)-I-tyrosine versus magnetic resonance imaging in the diagnosis of recurrent gliomas. Neurosurgery 2005; 57: 505-511; discussion 505-511.

75. Weber DC, Zilli T, Buchegger F et al. [(18)F]Fluoroethyltyrosine- positron emission tomography-guided radiotherapy for high-grade glioma. Radiat Oncol 2008; 3: 44.

76. Karunanithi S, Sharma P, Kumar A et al. Comparative diagnostic accuracy of contrast-enhanced MRI and (18)F-FDOPA PET-CT in recurrent glioma. Eur Radiol 2013; 23: 2628-2635.

77. Popperl G, Gotz C, Rachinger W et al. Value of O-(2-[18F]fluoroethyl)- L-tyrosine PET for the diagnosis of recurrent glioma. Eur J Nucl Med Mol Imaging 2004; 31 : 1464-1470.

78. Galldiks N, Dunkl V, Stoffels G et al. Diagnosis of pseudoprogression in patients with glioblastoma using O-(2-[18F]fluoroethyl)-L-tyrosine PET. Eur J Nucl Med Mol Imaging 2015; 42: 685-695. 
79. Nihashi T, Dahabreh IJ, Terasawa T. Diagnostic accuracy of PET for recurrent glioma diagnosis: a meta-analysis. AJNR Am J Neuroradiol 2013; 34: 944-950, S941-911.

80. Suchorska B, Jansen NL, Linn J et al. Biological tumor volume in 18FET-PET before radiochemotherapy correlates with survival in GBM. Neurology 2015; 84: 710719.

81. Lopci E, Riva M, Olivari L et al. Prognostic value of molecular and imaging biomarkers in patients with supratentorial glioma. Eur J Nucl Med Mol Imaging 2017; 44: 1155-1164.

82. Bosnyak E, Michelhaugh SK, Klinger NV et al. Prognostic Molecular and Imaging Biomarkers in Primary Glioblastoma. Clin Nucl Med 2017; 42: 341-347.

83. Spence AM, Muzi M, Swanson KRetal. Regional hypoxia in glioblastoma multiforme quantified with [18F]fluoromisonidazole positron emission tomography before radiotherapy: correlation with time to progression and survival. Clin Cancer Res 2008; 14: 2623-2630.

84. Zagzag $D$, Zhong $H$, Scalzitti JM et al. Expression of hypoxia-inducible factor 1 alpha in brain tumors: association with angiogenesis, invasion, and progression. Cancer 2000; 88: 2606-2618.

85. Evans SM, Judy KD, Dunphy I et al. Hypoxia is important in the biology and aggression of human glial brain tumors. Clin Cancer Res 2004; 10: 8177-8184.

86. Hoogsteen IJ, Marres HA, van der Kogel AJ, Kaanders JH. The hypoxic tumour microenvironment, patient selection and hypoxia-modifying treatments. Clin Oncol (R Coll Radiol) 2007; 19: 385-396.

87. Ljungkvist AS, Bussink J, Kaanders JH et al. Hypoxic cell turnover in different solid tumor lines. Int J Radiat Oncol Biol Phys 2005; 62: 1157-1168.

88. Wack LJ, Monnich D, van Elmpt W et al. Comparison of [18F]-FMISO, [18F]-FAZA and [18F]-HX4 for PET imaging of hypoxia--a simulation study. Acta Oncol 2015; 54: 1370-1377.

89. van Loon J, Janssen MH, Ollers M et al. PET imaging of hypoxia using [18F]HX4: a phase I trial. Eur J Nucl Med Mol Imaging 2010; 37: 1663-1668.

90. Zegers CM, Hoebers FJ, van Elmpt W et al. Evaluation of tumour hypoxia during radiotherapy using [18F]HX4 PET imaging and blood biomarkers in patients with head and neck cancer. Eur J Nucl Med Mol Imaging 2016; 43: 2139-2146.

91. Dubois LJ, Lieuwes NG, Janssen MH et al. Preclinical evaluation and validation of [18F]HX4, a promising hypoxia marker for PET imaging. Proc Natl Acad Sci U S A 2011; 108: 14620-14625.

92. Diaz LA, Jr., Bardelli A. Liquid biopsies: genotyping circulating tumor DNA. J Clin Oncol 2014; 32: 579-586. 
93. Crowley E, Di Nicolantonio F, Loupakis F, Bardelli A. Liquid biopsy: monitoring cancer-genetics in the blood. Nat Rev Clin Oncol 2013; 10: 472-484.

94. Kros JM, Mustafa DM, Dekker LJ et al. Circulating glioma biomarkers. Neuro Oncol 2015; 17: 343-360.

95. Redzic JS, Ung TH, Graner MW. Glioblastoma extracellular vesicles: reservoirs of potential biomarkers. Pharmgenomics Pers Med 2014; 7: 65-77.

96. Rosenfeld N, Aharonov R, Meiri E et al. MicroRNAs accurately identify cancer tissue origin. Nat Biotechnol 2008; 26: 462-469.

97. Qu S, Guan J, Liu Y. Identification of microRNAs as novel biomarkers for glioma detection: a meta-analysis based on 11 articles. J Neurol Sci 2015; 348: 181-187.

98. Shi R, Wang PY, Li XY et al. Exosomal levels of miRNA-21 from cerebrospinal fluids associated with poor prognosis and tumor recurrence of glioma patients. Oncotarget 2015; 6: 26971-26981.

99. Evans SM, Putt M, Yang XY et al. Initial evidence that blood-borne microvesicles are biomarkers for recurrence and survival in newly diagnosed glioblastoma patients. J Neurooncol 2016; 127: 391-400.

100. Macarthur KM, Kao GD, Chandrasekaran $S$ et al. Detection of brain tumor cells in the peripheral blood by a telomerase promoter-based assay. Cancer Res 2014; 74: 2152-2159.

101. Muller C, Holtschmidt J, Auer $M$ et al. Hematogenous dissemination of glioblastoma multiforme. Sci Transl Med 2014; 6: 247ra101.

102. Francis G, Stein S. Circulating Cell-Free Tumour DNA in the Management of Cancer. Int J Mol Sci 2015; 16: 14122-14142.

103. Piccioni DE, Lanman RB, Nagy RJ et al. Analysis of cell-free circulating tumor DNA in patients with glioblastoma and other primary brain tumors. Journal of Clinical Oncology 2015; 33: 11072-11072.

104. Weaver KD, Grossman SA, Herman JG. Methylated tumor-specific DNA as a plasma biomarker in patients with glioma. Cancer Invest 2006; 24: 35-40.

105. Bettegowda C, Sausen M, Leary RJ et al. Detection of circulating tumor DNA in early- and late-stage human malignancies. Sci Transl Med 2014; 6: $224 \mathrm{ra} 224$.

106. Diehl F, Schmidt K, Choti MA et al. Circulating mutant DNA to assess tumor dynamics. Nat Med 2008; 14: 985-990.

107. Chen H, Li X, Li W, Zheng H. miR-130a can predict response to temozolomide in patients with glioblastoma multiforme, independently of O6-methylguanineDNA methyltransferase. J Transl Med 2015; 13: 69.

108. Kushwaha D, Ramakrishnan V, Ng K et al. A genome-wide miRNA screen revealed miR-603 as a MGMT-regulating miRNA in glioblastomas. Oncotarget 2014; 5: 4026-4039. 
109. Verbeek B, Southgate TD, Gilham DE, Margison GP. O6-Methylguanine-DNA methyltransferase inactivation and chemotherapy. Br Med Bull 2008; 85: 17-33.

110. Turcan S, Rohle D, Goenka A et al. IDH1 mutation is sufficient to establish the glioma hypermethylator phenotype. Nature 2012; 483: 479-483.

111. Capper D, Simon M, Langhans CD et al. 2-Hydroxyglutarate concentration in serum from patients with gliomas does not correlate with IDH1/2 mutation status or tumor size. Int J Cancer 2012; 131: 766-768.

112. Husain H, Savage W, Grossman SA et al. Pre- and post-operative plasma glial fibrillary acidic protein levels in patients with newly diagnosed gliomas. J Neurooncol 2012; 109: 123-127.

113. Todaro L, Christiansen S, Varela M et al. Alteration of serum and tumoral neural cell adhesion molecule (NCAM) isoforms in patients with brain tumors. J Neurooncol 2007; 83: 135-144.

114. Ilhan-Mutlu A, Wagner L, Widhalm $G$ et al. Exploratory investigation of eight circulating plasma markers in brain tumor patients. Neurosurg Rev 2013; 36: 4555; discussion 55-46.

115. Hormigo A, Gu B, Karimi S et al. YKL-40 and matrix metalloproteinase-9 as potential serum biomarkers for patients with high-grade gliomas. Clin Cancer Res 2006; 12: 5698-5704.

116. Sreekanthreddy P, Srinivasan H, Kumar DM et al. Identification of potential serum biomarkers of glioblastoma: serum osteopontin levels correlate with poor prognosis. Cancer Epidemiol Biomarkers Prev 2010; 19: 1409-1422.

117. Albulescu R, Codrici E, Popescu ID et al. Cytokine patterns in brain tumour progression. Mediators Inflamm 2013; 2013: 979748.

118. Reynes G, Vila V, Martin M etal.Circulating markers of angiogenesis, inflammation, and coagulation in patients with glioblastoma. J Neurooncol 2011; 102: 35-41.

119. Schneider T, Sailer M, Ansorge $S$ et al. Increased concentrations of transforming growth factor beta 1 and beta2 in the plasma of patients with glioblastoma. J Neurooncol 2006; 79: 61-65.

120. Marusyk A, Almendro V, Polyak K. Intra-tumour heterogeneity: a looking glass for cancer? Nat Rev Cancer 2012; 12: 323-334.

121. Hambardzumyan D, Bergers G. Glioblastoma: Defining Tumor Niches. Trends Cancer 2015; 1: 252-265.

122. Aerts HJ. The Potential of Radiomic-Based Phenotyping in Precision Medicine: $A$ Review. JAMA Oncol 2016; 2: 1636-1642.

123. Limkin EJ, Sun R, Dercle L et al. Promises and challenges for the implementation of computational medical imaging (radiomics) in oncology. Ann Oncol 2017; 28 : 1191-1206. 


\section{Chapter 3 - Deciphering the glioblastoma phenotype by Computed Tomography radiomics}

Inge Compter, Maikel Verduin, Zhenwei Shi, Henry C. Woodruff, Robert Jan Smeenk, Tom Rozema, Ralph T.H. Leijenaar, René Monshouwer, Daniëlle B.P. Eekers, Ann Hoeben, Alida A. Postma, Andre Dekker, Dirk De Ruysscher, Philippe Lambin, Leonard Wee

Radiother Oncol. 2021 Jul;160:132-139. 


\section{Abstract}

\section{Introduction}

Glioblastoma (GBM) is the most common malignant primary brain tumour which has, despite extensive treatment, a median overall survival of 15 months. Radiomics is the high-throughput extraction of large amounts of image features from radiographic images, which allows capturing the tumour phenotype in 3D and in a non-invasive way. In this study we assess the prognostic value of $C T$ radiomics for overall survival in patients with a GBM.

\section{Materials and methods}

Clinical data and pre-treatment CT images were obtained from 218 patients diagnosed with a GBM via biopsy who underwent radiotherapy +/- temozolomide between 2004 and 2015 treated at three independent institutes ( $n=93,62$ and 63). A clinical prognostic score (CPS), a simple radiomics model consisting of volume based score (VPS), a complex radiomics prognostic score (RPS) and a combined clinical and radiomics $(C+R) P S$ model were developed. The population was divided into three risk groups for each prognostic score and respective Kaplan-Meier curves were generated.

\section{Results}

Patient characteristics were broadly comparable. Clinically significant differences were observed with regards to radiation dose, tumour volume and performance status between datasets. Image acquisition parameters differed between institutes. The cross-validated c-indices were moderately discriminative and for the CPS ranged from 0.63 to 0.65 ; the VPS c-indices ranged between 0.52 and 0.61 ; the RPS c-indices ranged from 0.57 to 0.64 and the combined clinical and radiomics model resulted in c-indices of $0.59-0.71$.

\section{Conclusion}

In this study clinical and CT radiomics features were used to predict OS in GBM. Discrimination between low-, middle- and high-risk patients based on the combined clinical and radiomics model was comparable to previous MRI-based models. 


\section{Introduction}

Despite extensive treatment with surgery, radiotherapy, and concurrent and adjuvant chemotherapy, patients with glioblastoma (GBM) have a very poor prognosis with a median overall survival (mOS) of 15 months after diagnosis'. TA small subgroup of patients ( $\sim 5 \%)$ who survive for more than 3 years has been reported ${ }^{2}$. The wide range in OS underlines the need to estimate likely prognosis on the individual level in order to support personalized treatment.

Several clinical recursive partitioning analysis models (RPA) have previously been developed to compare glioma survival categories and obtain homogenous groups of patients to evaluate in clinical trials $\mathrm{s}^{3-6}$. These models only include clinical parameters such as performance status (PS) and age. In order to refine these models, the glioma phenotype has received considerable attention, thus identification of different phenotypes may hold important prognostic and predictive information in addition to RPA.

Radiomics has emerged as a novel component of clinical decision support systems ${ }^{7}$. It refers to the automated extraction of large amounts of imaging features from radiographic images. The radiomics hypothesis is that image-derived features capture information that is otherwise not visible ${ }^{8,9}$. Previous radiomics studies have demonstrated that quantitative assessment of non-invasive biomarkers holds prognostic information in numerous cancer types in addition to molecular and clinical characteristics ${ }^{8,10,11}$.

Radiomics may improve clinical decision making in glioma in addition to the known RPA models as there is evidence that it can differentiate between tumour grade, identify druggable mutations, and assess tumour response ${ }^{12-16}$. Moreover, radiomics offers the opportunity to retrieve imaging biomarkers for clinical trials and can be obtained retrospectively. Currently, radiomics research in GBM has primarily focused on MRI. However, GBM patients regularly receive $\mathrm{CT}$ scans either at first presentation or as part of their work-up for radiotherapy treatment planning.

Translating radiomics-based treatment outcome models into routine clinical use requires objective assessment of its prognostic performance in independent datasets. However, the majority of radiomics studies to date are lacking either detailed external validation or direct access to the individual patient ${ }^{17,18}$. The primary objective of this study was to examine the prognostic potential of clinical, tumour volume and CTderived radiomics features for OS in adult patients with biopsy confirmed GBM, treated by radiotherapy with or without temozolomide. 


\section{Materials and methods}

\section{Study population}

The study population was derived from routine care cases from the three participating institutions: MAASTRO Clinic, Radboud University Medical Centre (RadboudUMC) and the Verbeeten institute, all located in The Netherlands. Patient and treatment characteristics are summarized in Table 1. The inclusion criteria were: age at biopsy $\geq 18$ years, pathologically confirmed GBM at diagnosis, CT imaging and RT structure set available for analysis and treated with radiotherapy between January 2004 and December 2014. Only patients without a tumour resection (i.e. biopsy only) were included to create a clinically homogenous group and allow for evaluation of the tumour still present on the CT images. Clinical parameters and survival intervals from start of radiotherapy were extracted from electronic patient records. Follow-up consisted of clinical review and imaging every three months until death.

\section{Study design}

This work has been approved by internal review boards of three participating institutions as a retrospective chart review-based observational study (IRB/P0122). The study is a Transparent Reporting of a multivariable prediction model for Individual Prognosis Or Diagnosis (TRIPOD) Type $2 b$ investigation ${ }^{19}$.

We used internal-external cross-validation on multi-institutional data to investigate potential CT radiomics signature for OS in adult histopathologically biopsy confirmed GBM patients treated by radiotherapy. The "internal-external cross-validation" method of Steyerberg and Harrell offers a more rigorous validation than a purely randomized split into training and testing cohorts ${ }^{20}$. Due to the vast number of candidate features relative to the number of deaths, a step-by-step feature selection approach was used to reduce the number of features. We compared this "data-driven" radiomics model with (1) a knowledge-based clinical model consisting of well-known prognostic factors from an RPA model 6 and (2) a simple radiomics model consisting of only one feature - the primary tumour volume ${ }^{21}$.

\section{Image acquisition}

Treatment planning simulation CTs acquired prior to radiotherapy were used for the analysis. All CT scanners were used entirely without modifications to standard clinical procedure. Image acquisition parameters are presented in supplementary table 2 .

\section{Gross tumour volume identification}

Each gross tumour volume (GTV) was manually delineated by experienced radiation oncologists ( $n=9$, all with at least 5 - years of experience) using a radiotherapy planning MRI co-registered to the planning CT as reference image. The delineations were checked by a second experienced radiation oncologist. The planning MRI consisted of contrast-enhanced 1-3 mm slice T1-weighted images. The GTV was 
defined as the enhancing tumour in accordance with ESTRO-ACROP guidelines ${ }^{22}$. The delineation was performed using the participating institutes treatment planning systems (TPS) (XiO (version 4.0, updated to 4.51 in use in 2014), Elekta, Stockholm, Sweden; Eclipse (version 10), Varian Medical Systems, California, USA; Pinnacle 3, Philips Medical Systems, Fitchburg, USA). The CT images and delineated structures were exported from each TPS in Digital Imaging and Communications in Medicine (DICOM) format.

\section{Radiomics feature extraction}

Radiomics features were extracted using an open-source Python library pyRadiomics (v2.1.2) ${ }^{23}$. Images were resampled to $1 \mathrm{~mm}$ isotropic voxels. A total of 1093 features were extracted. These consisted of 13 morphology (shape) features, 17 intensityhistogram (first-order) features and 73 textural which includes e.g., Haralick features (gray-level co-occurrence matrix (GLCM), grey level size zone matrix (GLSZM), grey level run length matrix (GLRLM), and neighborhood grey tone difference matrix (NGTDM). The intensity and textural features were re-computed after Laplacian of Gaussian (LoG) filter with three kernel dimensions - $1 \mathrm{~mm}$ (90 features), $2 \mathrm{~mm}$ (90 features) and $3 \mathrm{~mm}$ (90 features). The features were re-computed following wavelet (coif1) decomposition at 8 levels, for every possible combination of either a high or low pass filter in each of three cardinal axes (720 features). Morphology features in pyRadiomics are independent of pre-processing filters. Binary masks for the GTV were generated from DICOM structure sets using the O-RAW extension library to pyRadiomics to process DICOM inputs ${ }^{24}$. The mathematical definition of each feature can be found in the pyRadiomics documentation and the pyRadiomics extraction settings file is included in the Supplementary Materials (supplementary file 1). Divergences between PyRadiomics and the Image Biomarker Standardization Initiative (IBSI) have been documented by the developers ${ }^{25}$.

\section{Model development}

A knowledge-based clinical prognostic score (CPS) was derived from a multivariable Cox regression of OS, using age in years and WHO PS as predictors. Cognitive function scores were not available in the present datasets ${ }^{6}$. Surgery type and tumour grade were not used because these were already applied as eligibility filters for the study. Categorical WHO-PS scores were re-coded in binary fashion as PS $\geq 1, \mathrm{PS} \geq 2$, etc. Binary variables containing less than 10 occurrences were excluded.

Next, a GTV prognostic score (VPS) was evaluated using a single radiomics feature, tumour volume. This follows the recommendation of Welch et $a^{21}$. to test accepted clinical factors that could yield similar prognostic performance as more complex signatures.

A radiomics prognostic score (RPS) was developed with a "data-driven" approach by sequentially reducing the number of candidate radiomics features within the 
training set. Each extracted radiomics feature was normalized by a Yeo-Johnson transformation ${ }^{26}$ followed by centering (mean $=0$ ) and scaling (sample standard deviation $=1$ ) according to the training set. The same steps were applied in the validation set without any re-computation.

Finally, the CPS model and RPS model were combined in a clinical- and radiomics prognostic score $((C+R) P S)$ model.

\section{Feature selection}

Since the variables for the CPS were knowledge-based, and the VPS has one feature, no feature selection was applied to these models. A univariate association with OS was performed in order to confirm no important clinical features were missed.

As radiomics features are known to change significantly due to differences in scanner model, image acquisition or reconstruction settings, we used the post-reconstruction ComBat harmonisation method to harmonise features extracted from images acquired across the different institutes ${ }^{27}$. No test-retest to eliminate intensity-based and textural features with poor repeatability was performed as there was no GBM CT test-retest study available at time of writing.

The feature selection approach for the RPS is shown schematically in Figure 1. One thousand unique bootstrap samples were drawn up from the entire cohort. In each bootstrap sample, the findCorrelation function in the $\mathrm{R}$ caret library ${ }^{28}$ was used to minimize the number of pairwise feature correlations greater than 0.90 or less than -0.90 ; this removes the highly correlated features without looking at the survival outcome. Next, least absolute shrinkage (LASSO ${ }^{29}$ embedded with Cox regression was used to count how many times individual features were retained; this was done with 5 -fold internal cross-validation with the default model tuning grid from $\mathrm{R} \mathrm{glmnet}{ }^{30}$. The top 10 features were arbitrarily retained, since the frequency of selected features decayed rapidly (Figure 1a). With the top ten features, a stepwise backward Cox regression was applied on the same 1000 bootstrap samples, and counted the frequency of unique radiomics signatures (combinations of one or more features) that were retained by the aforementioned stepwise regression. The top most frequent signature was arbitrarily selected as the RPS model, however there is no universal agreement on which signature to choose (Figure 1b).

\section{Model validation and risk grouping}

The general procedure recommended in TRIPOD for model development and validation was applied ${ }^{19}$. Using data from the three centres the prognostic performance using the internal-external method, with each centre being left out in turn, was estimated. For prognostic signatures, the global coefficients using all three datasets combined were determined and an over-optimism correction by the bootstrapping method was applied ${ }^{20}$. The same 1000 bootstrap samples for feature 
selection were also used for optimism estimation. The Harrell concordance index (c-index $)^{31}$ was used as measure of discrimination.

For clinical relevance, prognostic scores were used to divide the population into three risk groups - the best prognosis $25 \%$, the worst prognosis $25 \%$ and the remainder ${ }^{32}$. Survival curves for the risk group are presented as Kaplan-Meier (KM) plots.

\section{Statistical analysis}

All analyses were performed in R statistical software (V3.6.1, https://www.R-project. org/). For differences in patient characteristics, the appropriate two-sided hypothesis test was applied; standard Z-test for proportions of continuous variables, unpaired Wilcoxon test for median and range, and exact Fisher test for categorical variables. Univariate association with OS was used to confirm that important clinical features had not been overlooked. OS was analysed from commencement of radiotherapy until death or loss to follow-up (right censored) using the coxph function in the package survival ${ }^{33}$ and c-indices were obtained from the survcomp package ${ }^{34}$. Survival outcomes were compared between risk groups using the Kaplan-Meier survival analysis (log-rank test with one-sided $p$ value). In addition to the c-index and KM curves, we examined the distribution of prognostic scores generated by the model (namely, median and interquartile range) as well as regression of the prognostic scores themselves against OS as indication of the calibration slope, in accordance with Royston and Altman ${ }^{32}$ to check for discrimination, calibration and model fit. 


\section{Results}

Patient and treatment characteristics are summarized in Table 1. There were no statistically significant differences between the cohorts for gender, age at biopsy, or treatment. Significant and clinically relevant differences were observed for radiation dose, primary tumour volume and WHO-PS scores. The proportion of 1-year OS after the start of radiotherapy was not significantly different between cohorts. In univariate analysis, age, WHO-PS $\geq 1$ and WHO-PS $\geq 2$ were strongly associated with OS ( $p<$ 0.005 after Bonferroni correction). The treating clinic was marginally associated with OS (HR 1.2; 95\% Cl 0.99-1.3, $\mathrm{p}=0.0067$ ). Tumour location (e.g. frontal, parietal, occipital or temporal) was not strongly associated with OS (data not shown). There were no pairwise Spearman correlations stronger than \pm 0.33 between any of the features present in the RPS and CPS models.

Prognostic performance was quantified as c-indices for each of the four models, through internal-external cross-validation with each centre alternately left out, and with all subjects pooled together (Table 2). The CPS returned c-indices ranging from 0.63 to 0.65 in cross-validation, which was also consistent with a model trained on pooled subjects (apparent c-index 0.66, optimism-corrected c-index 0.65 ). The estimated $95 \%$ confidence interval $(\mathrm{Cl})$ was always above 0.50 . The tumour volume model (VPS) was uniformly inferior to CPS with a c-index which fluctuated from 0.52 to 0.61 in cross-validation. The estimated $95 \% \mathrm{Cl}$ generally included 0.50 . The radiomics model (RPS) resulted in an apparent c-index of 0.57 to 0.64 and an optimism-corrected $\mathrm{c}$-index of 0.64 . The $(\mathrm{C}+\mathrm{R}) \mathrm{PS}$ model returned $\mathrm{c}$-indices ranging from 0.59 to 0.71 in cross-validation. For both the RPS and $(\mathrm{C}+\mathrm{R}) \mathrm{PS}$ model the estimated $95 \% \mathrm{Cl}$ only included 0.50 when the model was trained in centres $1 \& 3$ and validated in centre 2 .

The distribution of prognostic scores and calibration slopes of each model are shown in Supplementary Table 3. Similar discrimination performance and appropriateness of model fit of the CPS and RPS models was confirmed by the calibration slope and distribution of their prognostic indices. The median and interquartile range of prognostic scores are broadly overlapping in each of the three datasets, suggestive of adequate discrimination and calibration in each. For the CPS models the scores at centres 2 and 3 have less heterogeneity, as evidenced by smaller interquartile range, due to the lack of any WHO-PS 0 patients in those datasets. Poor performance of the VPS model was confirmed by large changes in the calibration between centres and error in the calibration slope, such that we cannot rule out the actual slope is zero and therefore overall lack of model fit. Inspection of Schoenfeld residuals of the calibration line shows no evidence of the proportional hazards assumption being violated in any combination of model and dataset.

Model coefficients, estimated hazard ratio (HR) with $95 \%$ confidence interval, and $\mathrm{p}$-value for globally pooled models are shown in Table 3. Kaplan-Meier curves for the four different models (CPS, VPS, RPS, $(C+R) P S)$ trained on pooled data are shown in Figure 2. 
Table 1: Patient characteristics

\begin{tabular}{|c|c|c|c|}
\hline & $\begin{array}{l}\text { Centre } 1 \\
(n=93)\end{array}$ & $\begin{array}{r}\text { Centre } 2 \\
(n=62)\end{array}$ & $\begin{array}{l}\text { Centre } 3 \\
(n=63)\end{array}$ \\
\hline Biological sex & $37(39.8 \%)$ & $21(33.9 \%) *(p=0.56)$ & $26(41.3 \%) *(p=0.98)$ \\
\hline $\begin{array}{l}\text { Age at biopsy } \\
\qquad \begin{array}{r}\text { Median (range) } \\
\text { Over } 70 \text { years of age }\end{array}\end{array}$ & $\begin{array}{l}63.9(21-86) \\
29(31.2 \%)\end{array}$ & $\begin{array}{c}60.8(18-77) *(p=0.37) \\
12(19.4 \%) *(p=0.15)\end{array}$ & $\begin{array}{c}63.6(40-80) *(p=0.98) \\
13(20.6 \%) *(p=0.20)\end{array}$ \\
\hline $\begin{array}{r}\text { WHO Performance } \\
0 \\
1 \\
2 \\
3 \text { or } 4 \\
\text { (missing) }\end{array}$ & $\begin{array}{c}29(31.2 \%) \\
45(48.4 \%) \\
16(17.2 \%) \\
3(3.2 \%) \\
-\end{array}$ & $\begin{array}{c}0(0 \%) \\
19(30.6 \%) \\
20(32.2 \%) \\
3(4.8 \%) \\
20 \\
*(p<0.01)\end{array}$ & $\begin{array}{c}0(0 \%) \\
41(65.1 \%) \\
21(33.3 \%) \\
1(1.6 \%) \\
- \\
*(p<0.01)\end{array}$ \\
\hline $\begin{array}{l}\text { Interval biopsy to RT } \\
\text { Median (range) days }\end{array}$ & $28(0-86)$ & $33(16-65) *(p=0.017)$ & $32(5-402) *(p=0.027)$ \\
\hline $\begin{array}{l}\text { Concurrent } \\
\text { temozolomide }\end{array}$ & $59(63.4 \%)$ & $38(61.3 \%) *(p=0.92)$ & $31(47.6 \%) *(p=0.073)$ \\
\hline $\begin{array}{l}\text { Tumor physical dose } \\
\text { Median (range) in Gy }\end{array}$ & $60(2-60)$ & $60(0-60) *(p=0.92)$ & $45(4-59.4) *(p<0.01)$ \\
\hline $\begin{array}{l}\text { Gross tumor volume } \\
\qquad \text { Median (range) in } \mathrm{cm}^{3}\end{array}$ & $51.9(0.5-194)$ & $48.2(1-222) *(p=0.52)$ & $\begin{array}{c}31.9(4-165) \\
*(p=0.01)\end{array}$ \\
\hline $\begin{array}{l}\text { Kaplan-Meier median } \\
\text { (range) overall survival } \\
\text { in days }\end{array}$ & $224(3-4221)$ & $162(4-4055) *(p=0.02)$ & $\begin{array}{l}181(32-1246) \\
*(p=0.25)\end{array}$ \\
\hline $\begin{array}{l}\text { Kaplan-Meier overall } \\
\text { survival at } 1 \text { year } \\
\text { (95\% confidence interval) }\end{array}$ & $\begin{array}{c}23.7 \% \\
(16.4-34.1) \%\end{array}$ & $\begin{array}{c}17.9 \% *(p=0.50) \\
(10.2-31.4) \%\end{array}$ & $\begin{array}{c}17.5 \% *(p=0.47) \\
(10.2-29.9) \%\end{array}$ \\
\hline
\end{tabular}


Table 2: Prognostic discrimination according to concordance index (c-index) with the 95\% confidence interval provided between parentheses. Cross-validation is by the internalexternal method quoted in the text and leaving one participating centre out in turn

\begin{tabular}{|c|c|c|c|}
\hline & $\begin{array}{l}\text { Apparent } \\
\text { c-index }\end{array}$ & $\begin{array}{c}\text { Validation } \\
\text { c-index }\end{array}$ & $\begin{array}{l}\text { Optimism- } \\
\text { corrected } \\
\text { c-index }\end{array}$ \\
\hline \multicolumn{4}{|l|}{ CPS model } \\
\hline Train in $1 \& 2$, test in 3 & $0.652(0.60-0.70)$ & $0.648(0.57-0.73)$ & - \\
\hline Train in $1 \& 3$, test in 2 & $0.651(0.60-0.70)$ & $0.654(0.56-0.74)$ & - \\
\hline Train in $2 \& 3$, test in 1 & $0.659(0.60-0.72)$ & $0.633(0.57-0.70)$ & - \\
\hline Train in pooled data & $0.657(0.61-0.70)$ & - & $0.651(0.60-0.69)$ \\
\hline \multicolumn{4}{|l|}{ VPS model } \\
\hline Train in $1 \& 2$, test in 3 & $0.541(0.49-0.60)$ & $0.609(0.53-0.69)$ & - \\
\hline Train in $1 \& 3$, test in 2 & $0.567(0.51-0.62)$ & $0.515(0.41-0.62)$ & - \\
\hline Train in $2 \& 3$, test in 1 & $0.569(0.50-0.63)$ & $0.553(0.49-0.62)$ & - \\
\hline & $0.554(0.49-0.62)$ & - & $0.547(0.48-0.61)$ \\
\hline \multicolumn{4}{|l|}{ RPS model } \\
\hline Train in $1 \& 2$, test in 3 & $0.659(0.61-0.71)$ & $0.598(0.52-0.68)$ & - \\
\hline Train in $1 \& 3$, test in 2 & $0.668(0.62-0.71)$ & $0.568(0.48-0.66)$ & - \\
\hline Train in $2 \& 3$, test in 1 & $0.641(0.58-0.70)$ & $0.639(0.59-0.69)$ & - \\
\hline Train in pooled data & $0.655(0.61-0.70)$ & - & $0.643(0.60-0.69)$ \\
\hline \multicolumn{4}{|l|}{$(C+R) P S$ model } \\
\hline Train in $1 \& 2$, test in 3 & $0.693(0.65-0.74)$ & $0.684(0.62-0.75)$ & - \\
\hline Train in $1 \& 3$, test in 2 & $0.703(0.66-0.74)$ & $0.589(0.48-0.70)$ & - \\
\hline Train in $2 \& 3$, test in 1 & $0.665(0.61-0.72)$ & $0.707(0.66-0.76)$ & - \\
\hline Train in pooled data & $0.691(0.65-0.73)$ & - & $0.689(0.65-0.73)$ \\
\hline
\end{tabular}




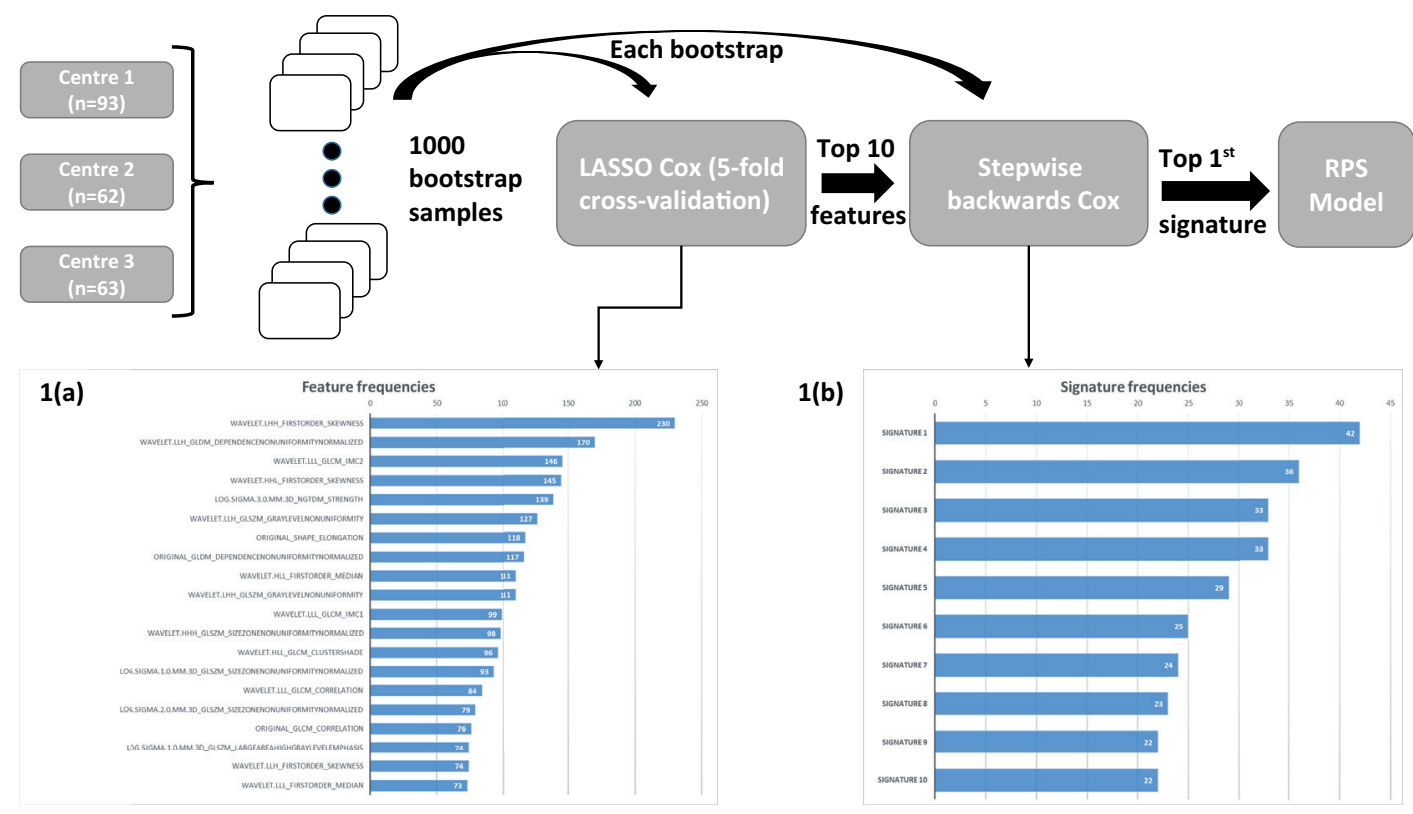

Fig. 1 (a-b). Feature selection approach for the RPS model. 

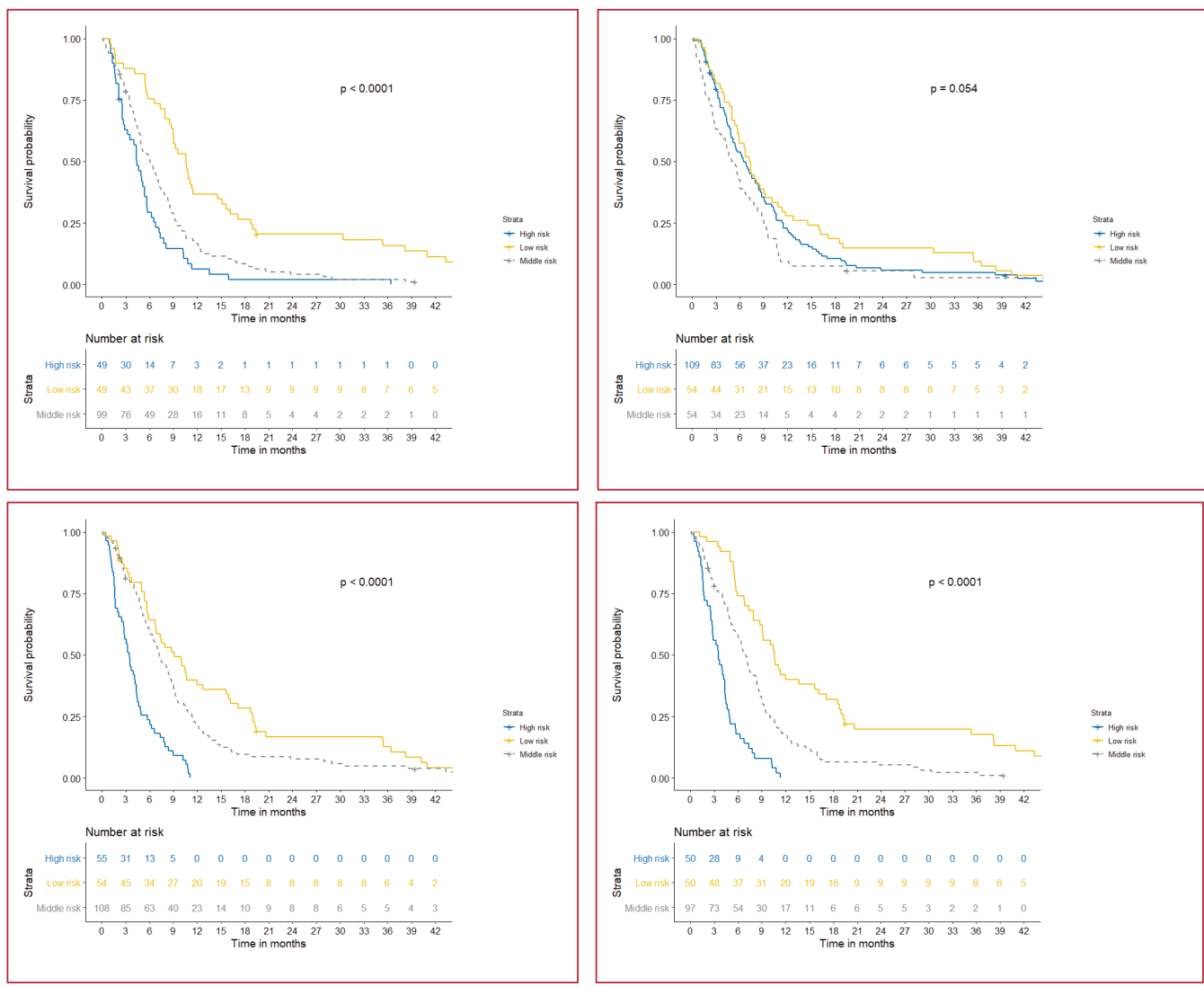

Fig. 2 (a-d). Kaplan-Meier analysis of overall survival of the complete study cohort. The population was divided into three risk groups for each prognostic score a) clinical prognostic score (CPS), b) volume based score (VPS), c) radiomics prognostic score (RPS), d) clinical and radiomics prognostic score $(C+R) P S$ 
Table 3: Model coefficients

\begin{tabular}{|c|c|c|c|c|}
\hline $\begin{array}{l}\text { Model } \\
\text { and } \\
\text { variables }\end{array}$ & $\begin{array}{l}\text { Cox } \\
\text { regression } \\
\text { coefficients }\end{array}$ & $\begin{array}{c}\text { Hazard } \\
\text { ratio (HR) } \\
\text { estimate }\end{array}$ & $\begin{array}{c}95 \% \\
\text { confidence } \\
\text { interval of HR }\end{array}$ & p-value \\
\hline \multicolumn{5}{|l|}{ CPS (pooled) } \\
\hline Age at biopsy & 0.035 & 1.04 & $(1.02-1.05)$ & $<0.001$ \\
\hline WHO-PS $\geq 1$ & 0.302 & 1.35 & $(0.88-2.08)$ & 0.167 \\
\hline WHO-PS $\geq 2$ & 0.510 & 1.66 & $(1.20-2.30)$ & 0.002 \\
\hline \multicolumn{5}{|l|}{ VPS (pooled) } \\
\hline Tumor volume & 0.099 & 1.10 & $(0.96-1.27)$ & 0.2 \\
\hline \multicolumn{5}{|l|}{ RPS (pooled) } \\
\hline $\begin{array}{l}\text { Wavelet-LHH: } \\
\text { Intensity Skewness }\end{array}$ & 0.263 & 1.30 & $(0.09-2.96)$ & 0.003 \\
\hline $\begin{array}{l}\text { Wavelet-LLH: } \\
\text { GLDM DependenceNon } \\
\text { UniformityNormalized }\end{array}$ & 0.304 & 1.35 & $(0.112 .85)$ & 0.004 \\
\hline Wavelet-LLL : GLCM IMC2 & 0.291 & 1.34 & $(0.08-3.87)$ & $<0.001$ \\
\hline $\begin{array}{l}\text { Wavelet-HHL: } \\
\text { Intensity Skewness }\end{array}$ & 0.396 & 1.49 & $(0.15-2.64)$ & 0.008 \\
\hline $\begin{array}{l}\text { Wavelet-LLH: GLSZM } \\
\text { GrayLevelNonUniformity }\end{array}$ & 0.261 & 1.30 & $(0.10-2.71)$ & 0.006 \\
\hline Shape : Elongation & 0.128 & 1.14 & $(0.08-1.57)$ & 0.116 \\
\hline $\begin{array}{l}\text { Wavelet-HLL: } \\
\text { Intensity Median }\end{array}$ & -0.140 & 0.87 & $(0.07-1.98)$ & 0.048 \\
\hline
\end{tabular}

(Abbreviations: WHO - World Health Organization; PS - Performance status; GLDM - Grey-level Difference Matrix; GLCM - Grey-level co-occurrence matrix; IMC - Information Measure of Content; GLSZM - Gray-level size zone matrix) 


\section{Discussion}

Identification of patients with a poor prognosis is of vital clinical importance as it may be questioned if it would be beneficial to offer these patient chemo-radiation, which may significantly decrease quality of life, or discuss best supportive care. This decision is currently primarily based on performance status, but this study demonstrates more factors, such as the GBM phenotype, should be considered. In the future these considerations should be implemented into decision support systems for $\mathrm{GBM}^{35}$. A steadily growing number of studies have been able to predict survival based on CT analysis in numerous types of cancer ${ }^{8,36-39}$. Skogen et al. have previously demonstrated that with a 2D CT texture analysis tumour heterogeneity correlated better with grade than size and attenuation did in glioma patients ${ }^{40}$. However, the present study is the first to investigate OS in patients with a GBM with CT-based radiomics. The CPS model based on age and WHO performance status and RPS model both demonstrated a moderate discriminative signature with an apparent c-index 0.66 , and a combined c-index of 0.69 , whereas theVPS only returned a c-index of 0.55 . Figure 2 demonstrates the discriminative power of the four models to separate the cohort into risk classes for survival. The assignment to the high-risk group according to the Cox models is associated with an increased Relative risk (RR) of mortality in the first 3 months compared to all the others (CPS: $\mathrm{RR}=2.03$; RPS: $\mathrm{RR}=2.46$; combined CPS+RPS: $R R=2.75$ ). The first three months is an arbitrarily chosen timepoint, however it is clinically meaningful for decision-making as most physicians will be very hesitant to start treatment if the life-expectancy of the patient is three months or shorter.

Since discrimination is preserved in every validation for the CPS model, it may be possible to update the model with new clinical parameters such that the calibration is augmented. The lack of discriminative power for the VPS model is noteworthy as it is generally accepted that a larger tumour volume is correlated with a worse prognosis ${ }^{41}$. This indicates that other factors should be accounted for. The distribution of the PIs for the RPS model (supplementary Table 3 ) does not suggest there is a major problem with the radiomics features being defined differently or having an incompatible set of units. Potential reasons for the lack of fit are the considerable heterogeneity in clinical- and imaging parameters between the three investigated cohorts or the need to recalibrate the models specifically for the brain. The residuals of the regression of the Pls to the outcome of interest, i.e. a Schoenfeld test, did not indicate that the proportional hazards assumption had become untenable in any of the aforementioned datasets.

The historically available recursive partitioning analyses have shown their prognostic significance in the characterization of glioma subgroups with respect to survival ${ }^{3-6}$. These classifications were limited to tumour grade, age, PS, extent of surgery and mental status. Table 1 shows there is clinical heterogeneity present between the three datasets. Despite our best efforts, information on the WHO-PS was missing in a significant portion of centre $2(32 \%)$. When comparing patient characteristics and OS between datasets we 
believe that there may be a selection bias present based on local neurosurgical clinical practice.

All of the CT scans used were acquired for simulation in routine image-guided radiotherapy planning (supplementary table 2). However, the CT scanners and the imaging protocols used in this study differed in regards to the acquisition parameters and contrast enhancement. It has previously been demonstrated that both image quality and repeatability is highly dependent on imaging protocol and CT vendor, potentially influencing our results ${ }^{42-44}$. In order to minimize protocol differences additional CT scanner quality assurance by means of phantom studies may prove to be necessary for reproducibility ${ }^{45}$. The volumes-of-Interest required were manually delineated by the treating physicians based on the ESTRO guideline ${ }^{22}$. However, interobserver variability cannot be excluded ${ }^{46}$. Automated segmentation based on $\mathrm{HU}$ for CT or artificial neural-network based methods for MR, could aid in reducing inter- and intra-observer variability and may also significantly influence feature reproducibility, but remains to be investigated. There are, to date, neither test-retest, multiple scanner series of the same subject nor inter-observer delineation $C T$ datasets specifically for GBM that might otherwise have been used during feature selection.

Although MRI is the preferred imaging modality for characterizing GBM in clinical practice, this study has focussed on re-using $C T$ scans acquired for radiotherapy treatment planning. A major advantage of $C T$ is that the grey-level values are expressed in $\mathrm{HU}$ which can be correlated with tissue density. For the delineation of GBM a fusion of $\mathrm{CT}$ images with MRI is preferred due to high resolution images and enhanced contrast within soft tissues ${ }^{47}$. However, unlike CT, MR gray-level values in regularly acquired sequences do not always directly correlate to the physical properties of tissue, but require a pre-processing normalization step ${ }^{48}$. This renders standard MRI sequences such as T1, $\mathrm{T} 2$ and Fluid-attenuated inversion recovery (FLAIR) unsuitable for radiomics analysis without pre-processing, resulting in a potential loss of information. Methods to reduce these issues include intensity normalization (e.g. gaussian and Z-score normalisation) and voxel re-slicing ${ }^{49-51}$. So far, several studies have investigated normalized MRI images by means of radiomics and demonstrated c-indices between 0.62 and 0.85 for multivariate models with either MR features alone or combined with clinical features such as age and performance status or molecular markers such as MGMT ${ }^{52-60}$. The combined $(C+R) P S$ model presented in this study returned an apparent c-index of 0.69 , which is comparable to the studies evaluating radiomics on MRI. More advanced MRI techniques such as Diffusion Weighted Imaging with Apparent Diffusion Coefficient maps, Diffusion Tensor Imaging, do directly allow for the assessment of the physiological properties of tissue and correlation with survival has been demonstrated ${ }^{61-63}$.

This present study has several limitations. All three cohorts included retrospectively collected data, in which the risk of aforementioned selection bias cannot be excluded. Moreover, not all clinical factors could be retrieved in all three datasets. As images were 
collected over a longer time period differences in image quality may exist, in order to retain sample size all available images were included. A larger prospectively collected dataset, including standardized homogenized imaging protocols, may be required in order to improve the performance of radiomics models. Decentralized methods for model development and model validation, where individual patient-level does not need to leave the institution, will be a boon to future radiomics modelling studies ${ }^{64,65}$. Known prognostic/predictive molecular markers such as MGMT (0-6-methylguanineDNA methyltransferase) promotor methylation and IDHI (isocitrate dehydrogenase 1) mutation were not available in all patients. A cohort membership model has been used to distinguish external validation that tests for replication or transferability in models for dichotomous outcomes, but there is presently no analogous test procedure for survival time analysis ${ }^{6,67}$. Our study excluded patients with a tumour debulking in order to create a homogeneous patient cohort and investigate the entire tumour volume at time of the planning CT. Confounding effects due to blood remnants after the biopsy or an open vs stereotactic biopsy may have influenced the results.

A major advantage of radiomics is that it is capable of evaluating the entire tumour volume, whereas biopsies could result in sampling errors as genetic profiles from one section of the tumour may not accurately reflect its whole genetic profile. Presently the molecular status does not influence treatment choice in GBM. However, awaiting the results of studies investigating the efficacy of targeted anticancer drugs, radiomics may support personalized treatment for GBM. In order for radiomics-based models to be universally applicable new models should be validated on large and qualitatively high-level datasets. Making datasets publicly available through websites such as Cancerdata (https://www.cancerdata.org/), the radiomics Imaging Archive (www.radiomicsimagingarchive.info) or the Cancer imaging archive (https://www. cancerimagingarchive.net/) should be highly encouraged.

\section{Conclusion}

This study was a TRIPOD type $2 \mathrm{~b}$ model development study in three independent datasets. Clinical and CT radiomics features were used to predict OS. Discrimination between low-, middle- and high-risk patients based on the combined clinical and radiomics model was comparable to previous MRI-based models. Future research should evaluate CT images of patients with a GBM longitudinally, compare CT features with MRI features, investigate a correlation between molecular markers and radiomics features, prospective collection of data and data sharing. Ultimately the objective of the radiomics field is to enhance clinical outcome by improved outcome prediction. This will most likely be achieved by combining all available information into one single model.

\section{Role of Funding Source}

The authors received no specific funding for this work 
1. Stupp R, Hegi ME, Mason WP, et al. Effects of radiotherapy with concomitant and adjuvant temozolomide versus radiotherapy alone on survival in glioblastoma in a randomised phase III study: 5-year analysis of the EORTC-NCIC trial. Lancet Oncol. 2009;10(5):459-466.

2. Tykocki T, Eltayeb M. Ten-year survival in glioblastoma. A systematic review. J Clin Neurosci. 2018 Aug(1532-2653).

3. Curran WJ, Jr., Scott CB, Horton J, et al. Recursive partitioning analysis of prognostic factors in three Radiation Therapy Oncology Group malignant glioma trials. J Natl Cancer Inst. 1993;85(9):704-710.

4. Mirimanoff RO, Gorlia T, Mason W, et al. Radiotherapy and temozolomide for newly diagnosed glioblastoma: recursive partitioning analysis of the EORTC 26981/22981-NCIC CE3 phase III randomized trial. J Clin Oncol. 2006;24(16):25632569.

5. Scott $C B$, Scarantino $C$, Urtasun $R$, et al. Validation and predictive power of Radiation Therapy Oncology Group (RTOG) recursive partitioning analysis classes for malignant glioma patients: a report using RTOG 90-06. Int J Radiat Oncol Biol Phys. 1998;40(1):51-55.

6. Li J, Wang M, Won M, et al. Validation and simplification of the Radiation Therapy Oncology Group recursive partitioning analysis classification for glioblastoma. Int J Radiat Oncol Biol Phys. 2011;81(3):623-630.

7. Lambin P, Zindler J, Vanneste BG, et al. Decision support systems for personalized and participative radiation oncology. Adv Drug Deliv Rev. 2017;109:131-153.

8. Aerts HJ, Velazquez ER, Leijenaar RT, et al. Decoding tumour phenotype by noninvasive imaging using a quantitative radiomics approach. Nat Commun. 2014;5:4006.

9. Gillies RJ, Kinahan PE, Hricak H. Radiomics: Images Are More than Pictures, They Are Data. Radiology. 2016;278(2):563-577.

10. Ou D, Blanchard P, Rosellini $S$, et al. Predictive and prognostic value of $C T$ based radiomics signature in locally advanced head and neck cancers patients treated with concurrent chemoradiotherapy or bioradiotherapy and its added value to Human Papillomavirus status. Oral Oncol. 2017;71:150-155.

11. Parmar C, Grossmann P, Rietveld D, Rietbergen MM, Lambin P, Aerts HJ. Radiomic Machine-Learning Classifiers for Prognostic Biomarkers of Head and Neck Cancer. Front Oncol. 2015;5:272.

12. Kickingereder $P$, Isensee $F$, Tursunova I, et al. Automated quantitative tumour response assessment of $M R I$ in neuro-oncology with artificial neural networks: a multicentre, retrospective study. Lancet, Oncol. 2019;May(1474-5488).

13. Tian $Q$, Yan LF, Zhang XA-O, et al. Radiomics strategy for glioma grading using texture features from multiparametric MRI. JMagn Reson Imaging. 2018 Dec(15222586 ). 
14. Skogen K, Schulz A, Dormagen JB, Ganeshan B, Helseth E, Server A. Diagnostic performance of texture analysis on MRI in grading cerebral gliomas. Eur, J Radiol. 2016;April(1872-7727).

15. Bahrami N, Hartman SJ, Chang YH, et al. Molecular classification of patients with grade II/III glioma using quantitative MRI characteristics. J Neurooncol. 2018;Sep(1573-7373).

16. Rathore S, Akbari HA-O, Rozycki M, et al. Radiomic MRI signature reveals three distinct subtypes of glioblastoma with different clinical and molecular characteristics, offering prognostic value beyond IDH1. Sci, Rep. 2018 Mar 23(2045-2322).

17. Traverso A, Wee L, Dekker A, Gillies R. Repeatability and Reproducibility of Radiomic Features: A Systematic Review. Int J Radiat Oncol Biol Phys. 2018;102(4):1143-1158.

18. Yip SS, Aerts HJ. Applications and limitations of radiomics. Phys Med Biol. 2016;61(13):150-166.

19. Collins GS, Reitsma JB, Altman DG, Moons KG. Transparent reporting of a multivariable prediction model for individual prognosis or diagnosis (TRIPOD): the TRIPOD statement. Br J Cancer. 2015;112(2):251-259.

20. Steyerberg EW, Harrell FE, Jr. Prediction models need appropriate internal, internal-external, and external validation. J Clin Epidemiol. 2016;69:245-247.

21. Welch ML, Mclntosh C, Haibe-Kains B, et al. Vulnerabilities of radiomic signature development: The need for safeguards. Radiother Oncol. 2019;130:2-9.

22. Niyazi M, Brada M, Chalmers AJ, et al. ESTRO-ACROP guideline "target delineation of glioblastomas". Radiother, Oncol. 2016;Jan(1879-0887).

23. van Griethuysen JJM, Fedorov A, Parmar C, et al. Computational Radiomics System to Decode the Radiographic Phenotype. Cancer, Res. 2017;Nov 1(1538-7445).

24. Shi Z, Traverso A, van Soest J, Dekker A, Wee L. Technical Note: Ontology-guided radiomics analysis workflow (O-RAW). Med, Phys. 2019;Dec(2473-4209).

25. Zwanenburg AA-O, Vallières MA-O, Abdalah MA-O, et al. The Image Biomarker Standardization Initiative: Standardized Quantitative Radiomics for HighThroughput Image-based Phenotyping. Radiology. 2020(295(2):328-338).

26. Yeo I-K, Johnson RA. A New Family of PowerTransformations to Improve Normality or Symmetry. Biometrika. 2000;87(4):954-959.

27. Johnson WE, Li C, Rabinovic A. Adjusting batch effects in microarray expression data using empirical Bayes methods. Biostatistics. 2006;8(1):118-127.

28. Kuhn M. Caret package. Journal of Statistical Software. 2008;28(5).

29. Tibshirani R. THE LASSO METHOD FOR VARIABLE SELECTION IN THE COX MODEL. Statistics in Medicine. 1997;16(4):385-395.

30. Simon N, Friedman J, Hastie T, Tibshirani R. Regularization Paths for Cox's Proportional Hazards Model via Coordinate Descent. J Stat Softw. 2011;39(5):1-13.

31. Harrell FE, Jr., Lee KI Fau - Mark DB, Mark DB. Multivariable prognostic models: issues in developing models, evaluating assumptions and adequacy, and measuring and reducing errors. Stat Med. 1996;15(4):361-387. 
32. Royston P, Altman DG. External validation of a Cox prognostic model: principles and methods. BMC Med Res Methodol. 2013;13:33.

33. M. TT, M. GP. Modeling Survival Data: Extending the Cox Model. New York: Springer; 2000.

34. Schroder MS, Culhane Ac Fau - Quackenbush J, Quackenbush J Fau - HaibeKains B, Haibe-Kains B. survcomp: an R/Bioconductor package for performance assessment and comparison of survival models. Bioinformatics. 2011;27(22):32063208.

35. Walsh S, de Jong EEC, van Timmeren JE, et al. Decision Support Systems in Oncology. JCO Clin Cancer Inform. 2019;3:1-9.

36. Parmar C, Rios Velazquez E, Leijenaar R, et al. Robust Radiomics feature quantification using semiautomatic volumetric segmentation. PLoS One. 2014;9(7):e102107.

37. Larue $R$, Klaassen $R$, Jochems $A$, et al. Pre-treatment $C T$ radiomics to predict 3-year overall survival following chemoradiotherapy of esophageal cancer. Acta, Oncol. 2018;Nov(1651-226X).

38. Huang YQ, Liang $\mathrm{CH}$, He $\mathrm{L}$, et al. Development and Validation of a Radiomics Nomogram for Preoperative Prediction of Lymph Node Metastasis in Colorectal Cancer. J Clin Oncol. 2016;Jun 20(1527-7755).

39. Ding J, Xing Z, Jiang Z, et al. CT-based radiomic model predicts high grade of clear cell renal cell carcinoma. Eur, J Radiol. 2018;Jun(1872-7727).

40. Skogen K, Ganeshan B Fau - Good C, Good C Fau - Critchley G, Critchley G Fau - Miles K, Miles K. Measurements of heterogeneity in gliomas on computed tomography relationship to tumour grade. J Neurooncol. 2013;Jan(1573-7373).

41. Ellingson BM, Abrey LE, Nelson SJ, et al. Validation of postoperative residual contrast-enhancing tumor volume as an independent prognostic factor for overall survival in newly diagnosed glioblastoma. Neuro Oncol. 2018;20(9):12401250.

42. Mackin D, Fave $X$, Zhang L, et al. Measuring Computed Tomography Scanner Variability of Radiomics Features. Invest Radiol. 2015;50(11):757-765.

43. Leijenaar RT, Carvalho S, Velazquez ER, et al. Stability of FDG-PET Radiomics features: an integrated analysis of test-retest and inter-observer variability. Acta Oncol. 2013;52(7):1391-1397.

44. Clarke LP, Nordstrom RJ, Zhang H, et al. The Quantitative Imaging Network: NCl's Historical Perspective and Planned Goals. Transl Oncol. 2014;7(1):1-4.

45. Lambin P, Leijenaar RTH, Deist TM, et al. Radiomics: the bridge between medical imaging and personalized medicine. Nat Rev Clin, Oncol. 2017 Dec(1759-4782 ).

46. Visser M, Muller DMJ, van Duijn RJM, et al. Inter-rater agreement in glioma segmentations on longitudinal MRI. Neuroimage, Clin. 2019(2213-1582).

47. Fiorentino A, Caivano R, Pedicini P, Fusco V. Clinical target volume definition for glioblastoma radiotherapy planning: magnetic resonance imaging and computed tomography. Clin Trans/ Oncol. 2013;15(9):754-758. 
48. Kumar V, Gu Y, Basu S, et al. Radiomics: the process and the challenges. Magn Reson Imaging. 2012;30(9):1234-1248.

49. Loizou CP, Petroudi S, Seimenis I, Pantziaris M, Pattichis CS. Quantitative texture analysis of brain white matter lesions derived from T2-weighted MR images in MS patients with clinically isolated syndrome. J Neuroradiol. 2015;42(2):99-114.

50. Depeursinge A, Foncubierta-Rodriguez A, Van De Ville D, Muller H. Threedimensional solid texture analysis in biomedical imaging: review and opportunities. Medical image analysis. 2014;18(1):176-196.

51. Madabhushi A, Udupa JK. New methods of MR image intensity standardization via generalized scale. Med Phys. 2006;33(9):3426-3434.

52. Xi YB, Guo F, Xu ZL, et al. Radiomics signature: A potential biomarker for the prediction of MGMT promoter methylation in glioblastoma. Journal of magnetic resonance imaging: JMRI. 2017.

53. Choi $Y$, Nam Y, Jang J, et al. Radiomics may increase the prognostic value for survival in glioblastoma patients when combined with conventional clinical and genetic prognostic models. Eur Radiol. 2020.

54. Prasanna P, Patel J, Partovi S, Madabhushi A, Tiwari P. Radiomic features from the peritumoral brain parenchyma on treatment-naive multi-parametric MR imaging predict long versus short-term survival in glioblastoma multiforme: Preliminary findings. Eur Radiol. 2016.

55. Chaddad A, Tanougast C. Extracted magnetic resonance texture features discriminate between phenotypes and are associated with overall survival in glioblastoma multiforme patients. Medical \& biological engineering \& computing. 2016;54(11):1707-1718.

56. Lao J, Chen Y, Li ZA-O, et al. A Deep Learning-Based Radiomics Model for Prediction of Survival in Glioblastoma Multiforme. Sci, Rep. 2017 Sep 4(2045-2322).

57. Chaddad A, Sabri S, Niazi T, Abdulkarim B. Prediction of survival with multi-scale radiomic analysis in glioblastoma patients. Med Biol Eng, Comput. 2018 Dec(17410444).

58. Liu Y, Zhang X, Feng $N$, et al. The effect of glioblastoma heterogeneity on survival stratification: a multimodal MR imaging texture analysis. Acta, Radiol. 2018 Oct(1600-0455).

59. Verduin M, Primakov S, Compter I, et al. Prognostic and Predictive Value of Integrated Qualitative and Quantitative Magnetic Resonance Imaging Analysis in Glioblastoma. Cancers. 2021;13, 722.

60. Chen X, Fang M, Dong D, et al. Development and Validation of a MRI-Based Radiomics Prognostic Classifier in Patients with Primary Glioblastoma Multiforme. Acad Radiol. 2019;26(10):1292-1300.

61. Hu LS, Ning S, Eschbacher JM, et al. Radiogenomics to characterize regional genetic heterogeneity in glioblastoma. Neuro Oncol. 2017;19(1):128-137.

62. Kickingereder P, Gotz M, Muschelli J, et al. Large-scale Radiomic Profiling of Recurrent Glioblastoma Identifies an Imaging Predictor for Stratifying AntiAngiogenic Treatment Response. Clin Cancer Res. 2016;22(23):5765-5771. 
63. Abrol S, Kotrotsou A, Salem A, Zinn PO, Colen RR. Radiomic Phenotyping in Brain Cancer to Unravel Hidden Information in Medical Images. Top Magn Reson Imaging. 2017;26(1):43-53.

64. Bogowicz MA-Ohoo, Jochems A, Deist TM, et al. Privacy-preserving distributed learning of radiomics to predict overall survival and HPV status in head and neck cancer. Sci Rep. 2020;10(1):4542.

65. Shi ZA-Ohoo, Zhovannik I, Traverso A, et al. Distributed radiomics as a signature validation study using the Personal Health Train infrastructure. Sci Data. 2019;6(1):218.

66. Debray TP, Vergouwe Y, Koffijberg H, Nieboer D, Steyerberg EW, Moons KG. A new framework to enhance the interpretation of external validation studies of clinical prediction models. J Clin Epidemiol. 2015;68(3):279-289.

67. van Soest J, Meldolesi E, van Stiphout R, et al. Prospective validation of pathologic complete response models in rectal cancer: Transferability and reproducibility. Med Phys. 2017;44(9):4961-4967. 
The PyRadiomics extraction parameter settings file (params.yaml)

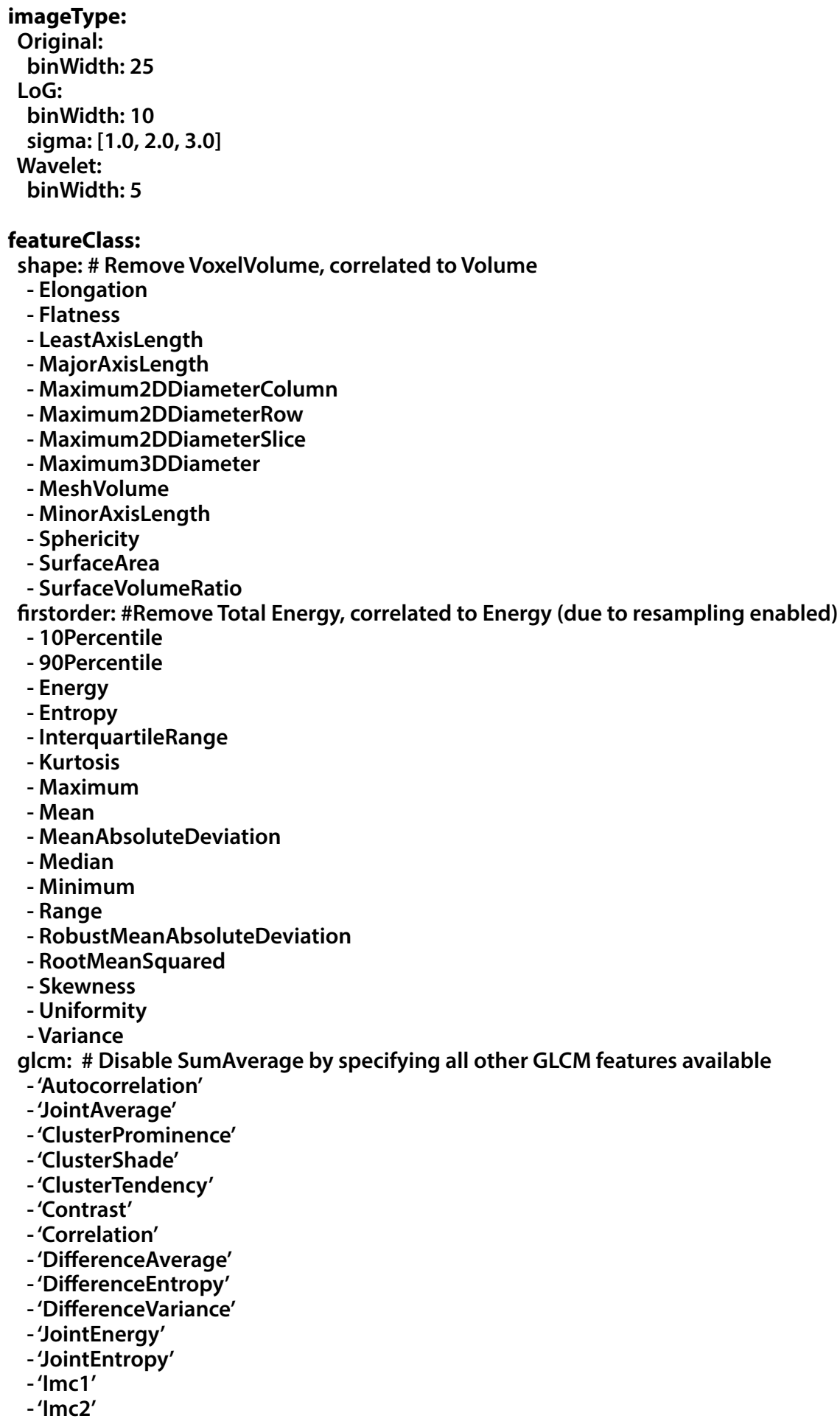




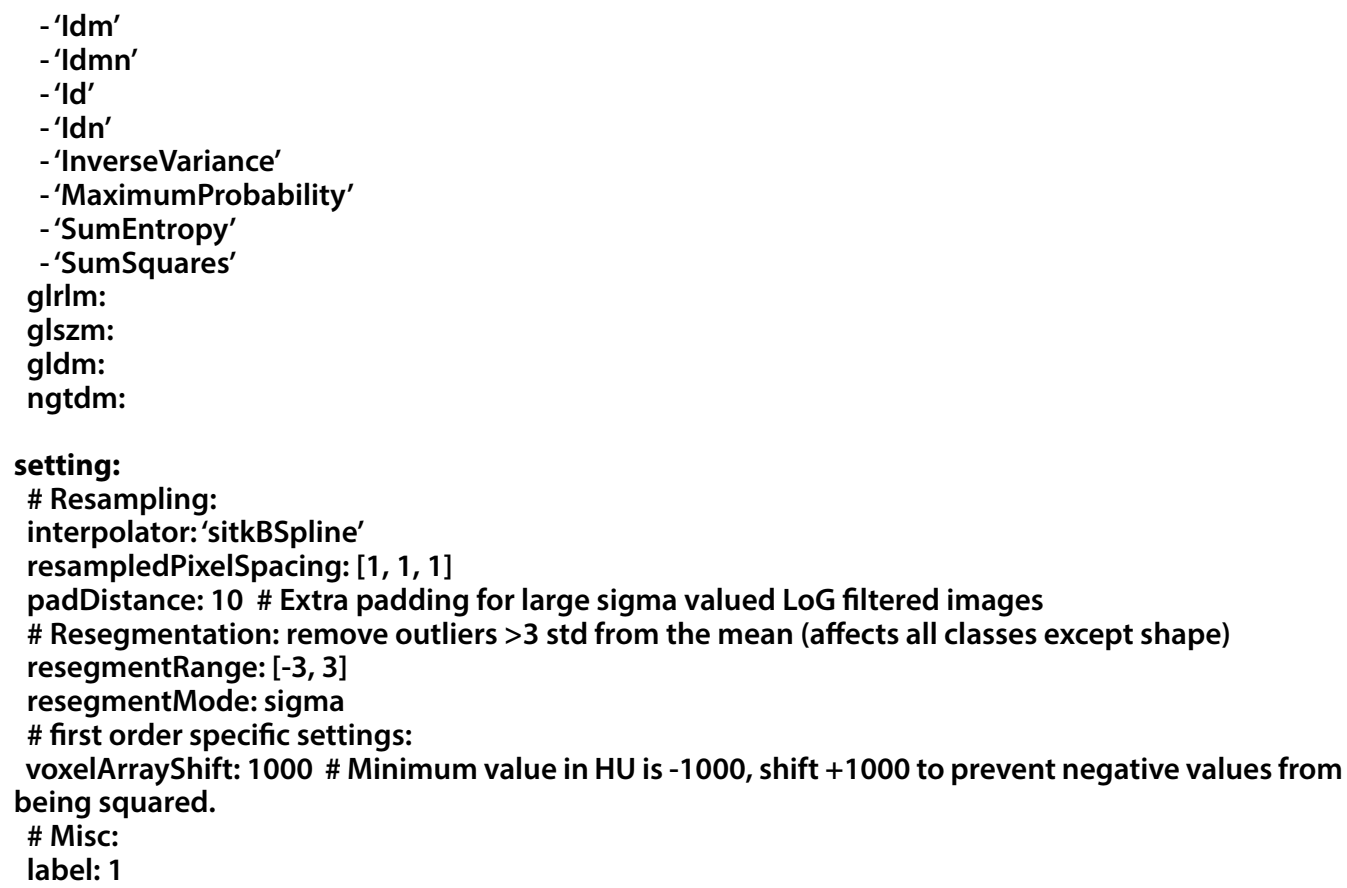


Supplementary Table S1 Radiomics features selected by LASSO Cox feature pooling, in order of decreasing frequency. For brevity, only the top fifteen most commonly occurring radiomics features are shown

\begin{tabular}{|l|c|}
\hline PyRadiomics feature label & Frequency \\
\hline wavelet.LHH_firstorder_Skewness & 230 \\
\hline wavelet.LLH_gldm_DependenceNonUniformityNormalized & 170 \\
\hline wavelet.LLL_glcm_Imc2 & 146 \\
\hline wavelet.HHL_firstorder_Skewness & 145 \\
\hline log.sigma.3.0.mm.3D_ngtdm_Strength & 139 \\
\hline wavelet.LLH_glszm_GrayLevelNonUniformity & 127 \\
\hline original_shape_Elongation & 118 \\
\hline original_gldm_DependenceNonUniformityNormalized & 117 \\
\hline wavelet.HLL_firstorder_Median & 111 \\
\hline wavelet.LHH_glszm_GrayLevelNonUniformity & 111 \\
\hline wavelet.LLL_glcm_Imc1 & 99 \\
\hline wavelet.HHH_glszm_SizeZoneNonUniformityNormalized & 98 \\
\hline wavelet.HLL_glcm_ClusterShade & 96 \\
\hline log.sigma.1.0.mm.3D_glszm_SizeZoneNonUniformityNormalized & 93 \\
\hline wavelet.LLL_glcm_Correlation & 84 \\
\hline
\end{tabular}


Supplementary Table S2 Image acquisition parameters for the three participating centres

\begin{tabular}{|c|c|c|c|}
\hline & Centre 1 & Centre 2 & Centre 3 \\
\hline Model & $\begin{array}{l}\text { Biograph } 40 \text { / } \\
\text { Sensation open/ } \\
\text { Sensation } 10 \\
\text { Siemens Healthcare, } \\
\text { Erlangen, Germany }\end{array}$ & $\begin{array}{l}\text { Brilliance Big Bore CT } \\
\text { scanner } \\
\text { Philips, Best, } \\
\text { The Netherlands }\end{array}$ & $\begin{array}{l}\text { Lightspeed RT16, } \\
\text { Hispeed NX, Optima } \\
\text { CT580 and Discovery } \\
\text { CT590, } \\
\text { General Electric } \\
\text { Healthcare, Chicago, } \\
\text { Illinois, United States }\end{array}$ \\
\hline $\begin{array}{l}\text { Resolution, pixel size } \\
(\mathrm{mm})\end{array}$ & $0.98 \times 0.98$ & $0.78 \times 0.78$ & $0.49 \times 0.49$ \\
\hline $\begin{array}{l}\text { Nominal tube potential } \\
(\mathrm{kVp})\end{array}$ & 120 & 120 & 120 \\
\hline Median exposure (mAs) & 270 (range $75-383$ ) & 362 (range $93-362$ ) & 299 (range $66-347$ ) \\
\hline Contrast enhancement & Yes & No & Yes \\
\hline Kernel & B31s/H31s & UB & Standard \\
\hline Reconstruction (mm) & 2 & $2-3$ & 2.5 \\
\hline
\end{tabular}


Supplementary Table 3 Distribution of prognostic scores and calibration slopes of models

\begin{tabular}{|c|c|c|c|c|}
\hline & $\begin{array}{l}\text { Median } \\
\text { prognostic } \\
\text { index }\end{array}$ & $\begin{array}{l}\text { Interquartile } \\
\text { range } \\
\text { (1st quartile; } \\
\text { 3rd quartile) }\end{array}$ & $\begin{array}{c}\text { * Regression } \\
\text { (calibration } \\
\text { slope) }\end{array}$ & $\begin{array}{l}\text { Std error of } \\
\text { calibration } \\
\text { slope }\end{array}$ \\
\hline CPS (pooled) & 0.024 & $0.60(-0.27 ; 0.33)$ & $* 1.00$ & 0.16 \\
\hline Centre 1 & -0.092 & $0.78(-0.52 ; 0.26)$ & 0.84 & 0.21 \\
\hline Centre 2 & 0.210 & $0.34(-0.14 ; 0.20)$ & 1.10 & 0.40 \\
\hline Centre 3 & 0.058 & $0.55(-0.18 ; 0.37)$ & 1.16 & 0.35 \\
\hline VPS (pooled) & 0.007 & $0.13(-0.07 ; 0.06)$ & $* 1.00$ & 0.71 \\
\hline Centre 1 & 0.025 & $0.13(-0.06 ; 0.07)$ & 1.28 & 1.21 \\
\hline Centre 2 & 0.018 & $0.07(-0.07 ; 0.00)$ & -0.06 & 1.18 \\
\hline Centre 3 & -0.034 & $0.13(-0.08 ; 0.05)$ & 3.64 & 1.58 \\
\hline RPS (pooled) & 0.049 & $0.69(-0.34 ; 0.35)$ & $* 1.00$ & 0.15 \\
\hline Centre 1 & 0.126 & $0.94(-0.46 ; 0.48)$ & 1.03 & 0.21 \\
\hline Centre 2 & 0.008 & $0.75(-0.40 ; 0.35)$ & 0.87 & 0.28 \\
\hline Centre 3 & 0.018 & $0.61(-0.32 ; 0.29)$ & 1.29 & 0.34 \\
\hline
\end{tabular}

*Note: Calibration slope of prognostic index (PI) in the pooled dataset must be 1.00 by its construction 


\section{Distribution of prognostic scores and calibration slopes}

The distribution of prognostic scores and calibration slopes of each model are shown in Supplementary Table 3. Similar discrimination performance and appropriateness of model fit of the CPS and RPS models was confirmed by the calibration slope and distribution of their prognostic indices. The median and interquartile range of prognostic scores are broadly overlapping in each of the three datasets, suggestive of adequate discrimination and calibration in each. For the CPS models the scores at centres 2 and 3 have less heterogeneity, as evidenced by smaller interquartile range, due to the lack of any WHO-PS 0 patients in those datasets. Poor performance of the VPS model was confirmed by large changes in the calibration between centres and error in the calibration slope, such that we cannot rule out the actual slope is zero and therefore overall lack of model fit. Inspection of Schoenfeld residuals of the calibration line shows no evidence of the proportional hazards assumption being violated in any combination of model and dataset. 


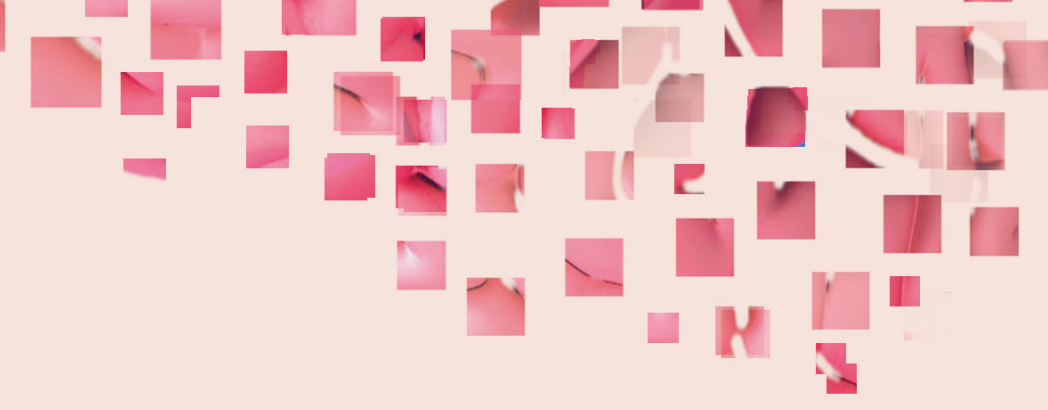

Part II

Ultra-high field Magnetic Resonance Imaging 


\section{Chapter 4 - Technical feasibility of integrating $7 \mathrm{~T}$ anatomical MRI in image-guided radiotherapy of glioblastoma: a preparatory study}

Inge Compter, Jurgen Peerlings, Daniëlle B.P. Eekers, Alida A. Postma, Dimo Ivanov, Christopher J. Wiggins, Pieter Kubben, Benno Küsters, Pieter Wesseling, Linda Ackermans, Olaf E.M.G. Schijns, Philippe Lambin, Aswin L. Hoffmann

MAGMA. 2016;29(3):591-603. 


\section{Abstract}

\section{Objectives}

7 Tesla (T) MRI has recently shown great potential for high-resolution soft-tissue neuroimaging and visualization of microvascularization in glioblastoma (GBM). We have designed a clinical trial to explore the value of 7T MRI for radiation treatment of GBM. For this clinical study we performed a preparatory study to investigate the technical feasibility of incorporating 7T MR images into the neurosurgical navigation and radiotherapy treatment planning (RTP) systems by qualitative and quantitative assessment of the image quality.

\section{Materials and methods}

The MR images were acquired with a Siemens Magnetom 7T whole-body scanner and a Nova Medical 32-channel head coil. 7T MRI pulse sequences included MP2RAGE, T2-SPACE, SPACE-FLAIR and GRE. A pilot study with 3 healthy volunteers and an anthropomorphic $3 \mathrm{D}$ phantom was used to assess image quality and geometrical image accuracy.

\section{Results}

The MRI scans were well tolerated by the volunteers. Susceptibility artefacts were observed in both the cortex and subcortical white matter at close proximity to airtissue interfaces. Regional loss of signal and contrast could be minimized by the use of dielectric pads. Image transfer and processing did not degrade image quality. The system-related spatial uncertainty of geometrical distortion-corrected MP2RAGE pulse sequences is $\leq 2 \mathrm{~mm}$.

\section{Objectives}

Integration of high-quality and geometrically-reliable 7TMRimages into neurosurgical navigation and RTP software is technically feasible and safe. 


\section{Introduction}

Glioblastoma (GBM) is the most common type of primary brain tumour having a peak incidence in the $6^{\text {th }}$ and $7^{\text {th }}$ decade. Patients with a GBM have an extremely poor prognosis with a median overall survival of 14.6 months ${ }^{1}$. The main goals of surgery are verification of histological diagnosis and reduction of any mass effect. A complete resection is nearly impossible because of the infiltrative nature of this disease and spread along white matter tracts ${ }^{2}$. Therefore, patients up to 70 years old with a good performance status are treated with adjuvant radiotherapy in combination with temozolomide to delay local tumour recurrence and increase overall survival. Radiotherapy usually consists of conventionally fractionated regimens, delivering a dose of 59.4-60 Gy in 6-7 weeks. Computed tomography (CT) images (1 mm slices) are commonly used for radiation therapy planning (RTP) because of the excellent spatial quality (i.e., no geometrical distortions) and the electron density information that is required for accurate dose calculations. In current RTP practice for GBM, contrast-enhanced (CE) CT images are co-registered with 1.5 or 3 Tesla (T) magnetic resonance (MR) images because the latter offer superior soft-tissue contrast over CT images. Subsequently, a gross tumour volume (GTV) is delineated based on the resection cavity and any residual disease visible on CE T1-weighted MRI scans. According to European guidelines a $2-3 \mathrm{~cm}$ isotropic margin is added to the GTV to encompass any non-enhancing tumour tissue and to establish the clinical target volume $(\mathrm{CTV})^{3}$. This margin is based on the fact that over $80 \%$ of recurrences occur within $2 \mathrm{~cm}$ of the GTV ${ }^{4,5}$. However, the isotropic margin does not take into account spatially varying tumour-growth dynamics in different brain tissues and tumour spread along white matter tracts, which evidently results in needlessly damaging healthy cells while leaving viable malignant cells outside the CTV ${ }^{6}$. A planning target volume (PTV) margin is added to account for systematic and random errors such as setup errors, inter- and intrafraction motion, but also uncertainty in image registration and delineation. Current clinically available MRI techniques are unable to adequately visualize tumour spread throughout the brain. Ultra-high field (UHF) (>3 T) MRI might be able to overcome these limitations, because the increased signalto-noise ratio (SNR) and susceptibility effects which allow for an increased spatial resolution and better contrast in comparison to clinically-used 1.5T and 3T MRI. However, the potential of UHF MRI for RTP of GBM has not been investigated so far.

With regard to neurosurgical planning, UHF MRI may benefit image-guided biopsies through identification of increased vascularity suggestive for tumour grade. Endothelial proliferation and increased microvascularization are key features for the diagnosis of GBM according to the WHO criteria ${ }^{7}$. An increase in susceptibility effects, as seen with UHF MRI in comparison to lower field strengths, results in novel contrast mechanisms on quantitative $\mathrm{T} 2{ }^{*}$-weighted images by which microvascularization with a vessel diameter as small as $100 \mu \mathrm{m}$ can be visualized ${ }^{8-11}$. 
We therefore hypothesize that 7T MRI allows for better delineation of the GTV of GBM due to improved visualization of microvascularization. We have designed a future clinical study to investigate this, which includes a 7T-image guided biopsy and RTP (clinicaltrials.gov NCT 02062372). In this paper we describe the technical aspects of the preparatory work for this clinical trial. If 7T MR images are to be used for neurosurgical and RTP purposes, a high spatial reliability is required. Although 7T MRI may hold promise to be included into neurosurgical navigation and RTP, it also presents technical challenges such as inhomogeneity of the transmit B1-field, an increased specific absorption rate (SAR) and geometrical distortions caused by increased static magnetic field (B0) inhomogeneity, tissue susceptibility differences and chemical shift effects ${ }^{12-14}$. In order to measure the geometric accuracy, a phantom study was conducted to quantify system-related geometrical image distortions. Furthermore, a pilot study with healthy volunteers was conducted to investigate whether the 7T MRI images meet the requirements for clinical application in RTP, such as visualization of brain anatomy structures on different sequences, differences in scanning times and patient tolerability of the scan. In this paper we report on the challenges and pitfalls we encountered in preparation of our clinical study, and present solutions we developed to quantify the system-related geometrical image distortions, optimize 7T MRI scanning protocols as well as the image transfer and processing workflow.

\section{Materials and methods}

\section{Pilot study}

A pilot study with healthy volunteers was conducted to assess the image quality in terms of field inhomogeneity and susceptibility artefacts and to optimize the pulse sequences and scanning protocols for RTP purposes. Prior to the 7T MRI scan all volunteers received detailed information regarding the purpose of the study and possible temporary sensory side effects due to the applied magnetic field. Written informed consent was obtained prior to participation. A total of three volunteers were recruited: two females ( 26 and 37 years old) and one male ( 29 years old). Subjects filled out a safety questionnaire prior to the scan regarding e.g. medication, claustrophobia and metallic objects, and were instructed not to move during the scanning procedure. They were positioned in a head-first supine position and dielectric pads were fixed on both sides of the subject's head next to the temporal lobes after which the head coil was placed. The dielectric pads contained a $25 \%$ suspension of barium titanate in deuterated water and were used to locally increase the transmit B1+ field to improve its homogeneity across the brain ${ }^{15}$. Cushions were placed under the knees to provide extra comfort. 


\section{Phantom study}

Geometrical distortion is a recognized problem in anatomical MRI sometimes resulting in pixel shifts of several millimetres, which is detrimental for application of MRI in image-guided interventions in neurosurgery and radiotherapy. Geometric inaccuracies originate from system (i.e., $B_{0}$-inhomogeneity, gradient field nonlinearity) or object (i.e., chemical shift, susceptibility effects) related causes, and can to some extent be corrected for by manufacturer-developed distortion correction methods and shimming procedures. As magnetic field inhomogeneity, chemical shift and susceptibility are proportional to $B_{0^{\prime}}$ the spatial inaccuracy increases with higher magnetic field strength. Hence, estimation of the geometrical distortion is essential before 7T MRI can be integrated into image-guided interventions.

For the assessment of system- and sequence-related geometrical image distortions, we used a dedicated 3D anthropomorphic skull phantom (CIRS Model 603A, Computerized Imaging Reference Systems, Inc., Norfolk, Virginia, United States; figure 1 and $2 \mathrm{a} \& 2 \mathrm{~b}$ ). This phantom is made up of a plastic-bone tissue substitute and soft-tissue equivalent material consisting of a water-based polyacrylamide (Zerdine ${ }^{\circledast}$, CIRS). The entire phantom is encased in a clear vacuum-formed plastic shell to protect the gel from desiccation. The cranial portion of the skull volume is filled with an orthogonal 3D grid of $3 \mathrm{~mm}$ diameter rods (reinforced Nylon) spaced $15 \mathrm{~mm}$ apart. The maximum 3D grid-size is $15 \times 12 \times 13.5 \mathrm{~cm}^{3}(\mathrm{AP} \times \mathrm{LR} \times \mathrm{SI})$, resulting in 436 measurable grid-intersection points. Since Nylon shows magnetic susceptibility properties similar to water (i.e., difference in susceptibility $<3 \mathrm{ppm}$ ), the phantom rods are expected not to induce artefacts and image abnormalities in either spinecho or gradient-echo sequences ${ }^{16}$. The phantom was placed in an ABS vacuumformed cradle to ensure reproducible placement within the scanner. Both CT and 7T MRI images were acquired in order to compare the geometrical distortions of both imaging modalities.

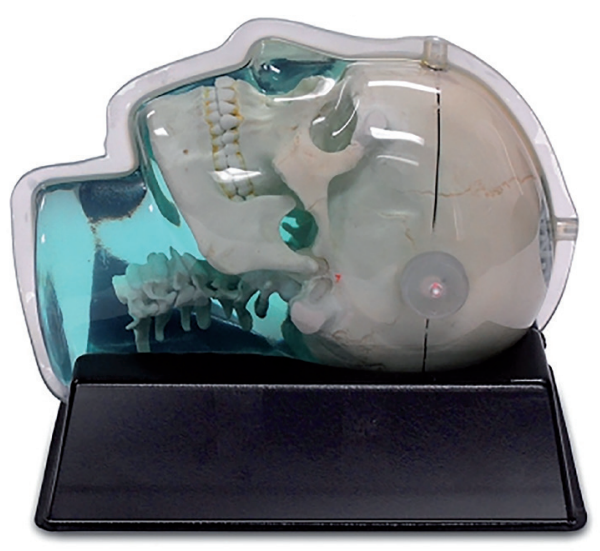

Fig. 1 Anthropomorphic skull phantom CIRS 603A. Source: Computerized Imaging Reference Systems, Inc (CIRS), viewed 22 November 2015 <http://www.cirsinc.com/ products/modality/99/mri-distortion-phantom-for-srs/ $>$ 

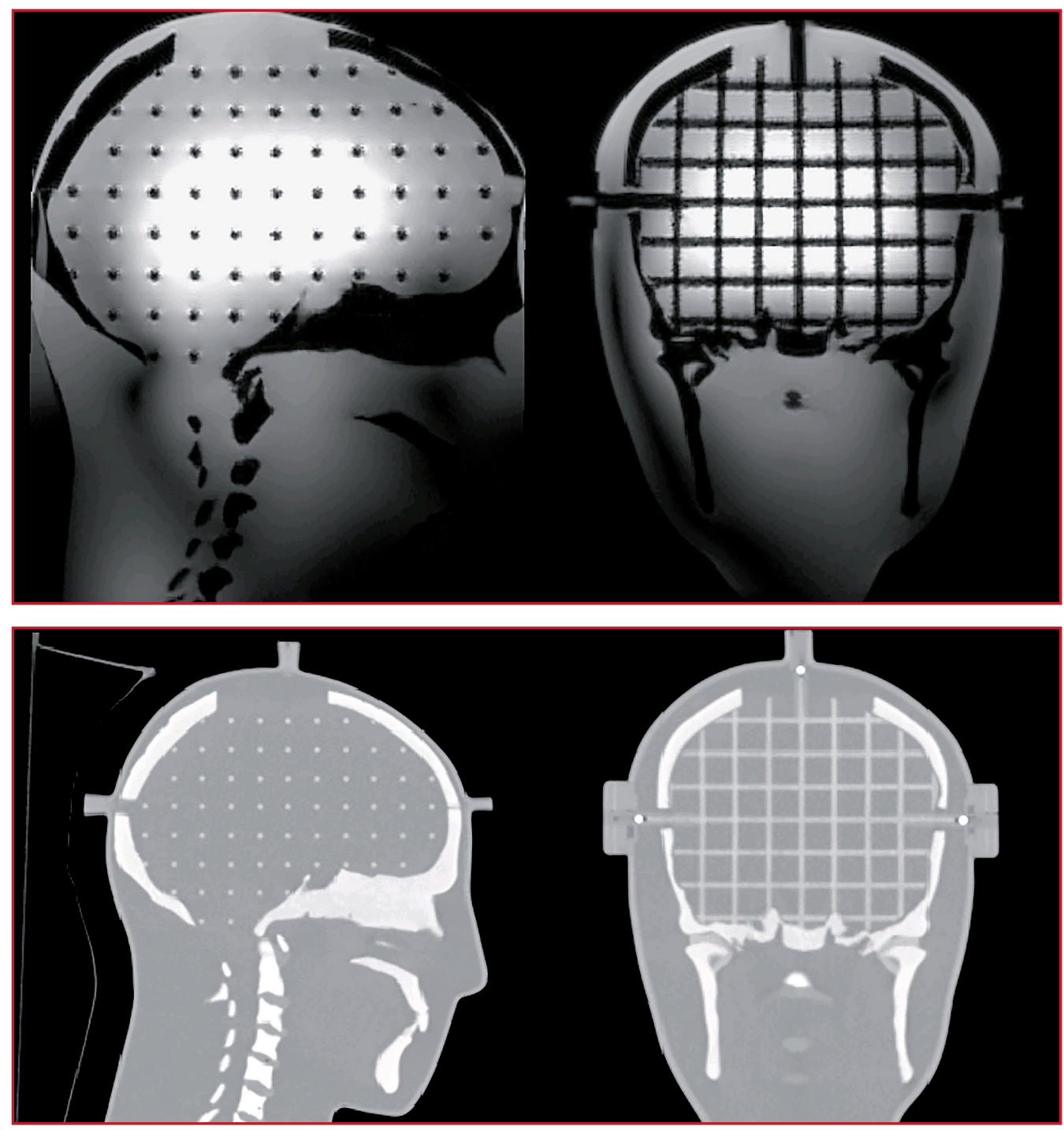

Fig. 2 Sagittal and coronal slice of the CIRS 603A phantom on CT (a), and 7T MRI (b) scan 


\section{Image acquisition}

For the phantom study, CT images (SOMATOM Sensation 10, Siemens Healthcare, Erlangen, Germany) were acquired at MAASTRO CLINIC with a $1 \mathrm{~mm}$ slice thickness, 306 slices, $50 \times 50 \mathrm{~cm} 2$ field of view, $140 \mathrm{kV}, 400 \mathrm{mAs}$.

For the pilot and phantom study, MR images were acquired at the Scannexus facility with a 7T whole-body scanner (Magnetom 7T, Siemens Healthcare, Erlangen, Germany) in combination with a 32-channel head coil (Nova Medical, Wilmington, United States). Pulse sequences included multi-echo gradient echo (GRE), magnetization-prepared rapid gradient-echo (MP2RAGE) ${ }^{17}$, T2- sampling perfection with application optimized contrasts using different flip angle evolution (T2-SPACE), and sampling perfection with application optimized contrasts using different flip angle evolution FLAIR (SPACE-FLAIR) (table 1).

Table 1 Scan parameters per 7TMRI pulse sequence

\begin{tabular}{|c|c|c|c|c|}
\hline & MP2RAGE & SPACE & SPACE-FLAIR & GRE \\
\hline Repetition time TR (ms) & 5000 & 4000 & 8000 & 33 \\
\hline Echo time TE (ms) & 2.5 & 283 & 302 & 2.5 \\
\hline Inversion time (ms) & $\begin{array}{c}\text { T11 } 900 \\
\text { T12 } 27500\end{array}$ & nvt & 2330 & nvt \\
\hline Field of View (mm) & $223 \times 223$ & $192 \times 192$ & $193 \times 206$ & $160 \times 223$ \\
\hline Nominal flip angle $\left({ }^{\circ}\right)$ & $5 \& 3$ & variable & variable & 11 \\
\hline Acquisition matrix (pixel) & $0 \backslash 320 \backslash 320 \backslash 0$ & $0 \backslash 320 \backslash 320 \backslash 0$ & $0 \backslash 256 \backslash 240 \backslash 0$ & $0 \backslash 320 \backslash 320 \backslash 0$ \\
\hline Bandwidth (Hz/pixel) & 248 & 372 & 383 & 290 \\
\hline Slices (n) & 240 & 288 & 208 & 208 \\
\hline Slice thickness (mm) & 0.7 & 0.6 & 0.8 & 0.7 \\
\hline Acquisition time (minutes) & 8.02 & 7.50 & 10.58 & 8.33 \\
\hline
\end{tabular}




\section{TMRI sequence selection and optimization}

Sequences were selected to highlight differences in tissue contrast ${ }^{18}$ and visualize microvascularization ${ }^{9,10}$. A standard method for obtaining $T_{1}$-contrast is the MPRAGE (magnetization-prepared rapid gradient-echo) sequence, which provides good greywhite matter contrast ${ }^{19}$. Due to inhomogeneities in both the transmit and receive radiofrequency (RF) fields, a newer variant (MP2RAGE) was used that generates two different images at different inversion times and allows for self-correction of the bias fields ${ }^{20}$.

The MP2RAGE sequence with optimized TR-FOCI inversion pulse was chosen for T1-weighted imaging instead of the MPRAGE because receive bias field could be

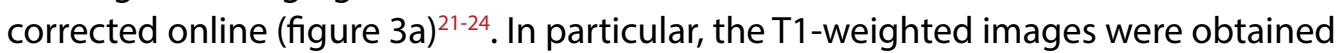
as the ratio of the two volumes acquired at different inversion times (INV1 and INV2), which minimizes the effect of $B 1$-variations through space. A quantitative $T_{1}$-map was calculated online by linear interpolation of the INV1 and INV2 images ${ }^{17}$. An optimized four-echo GRE was used instead of the typical single-echo GRE with a long echo time in order to correct for B1-inhomogeneities and obtain quantitative $\mathrm{T}_{2}{ }^{*}$ images (figure 3b).

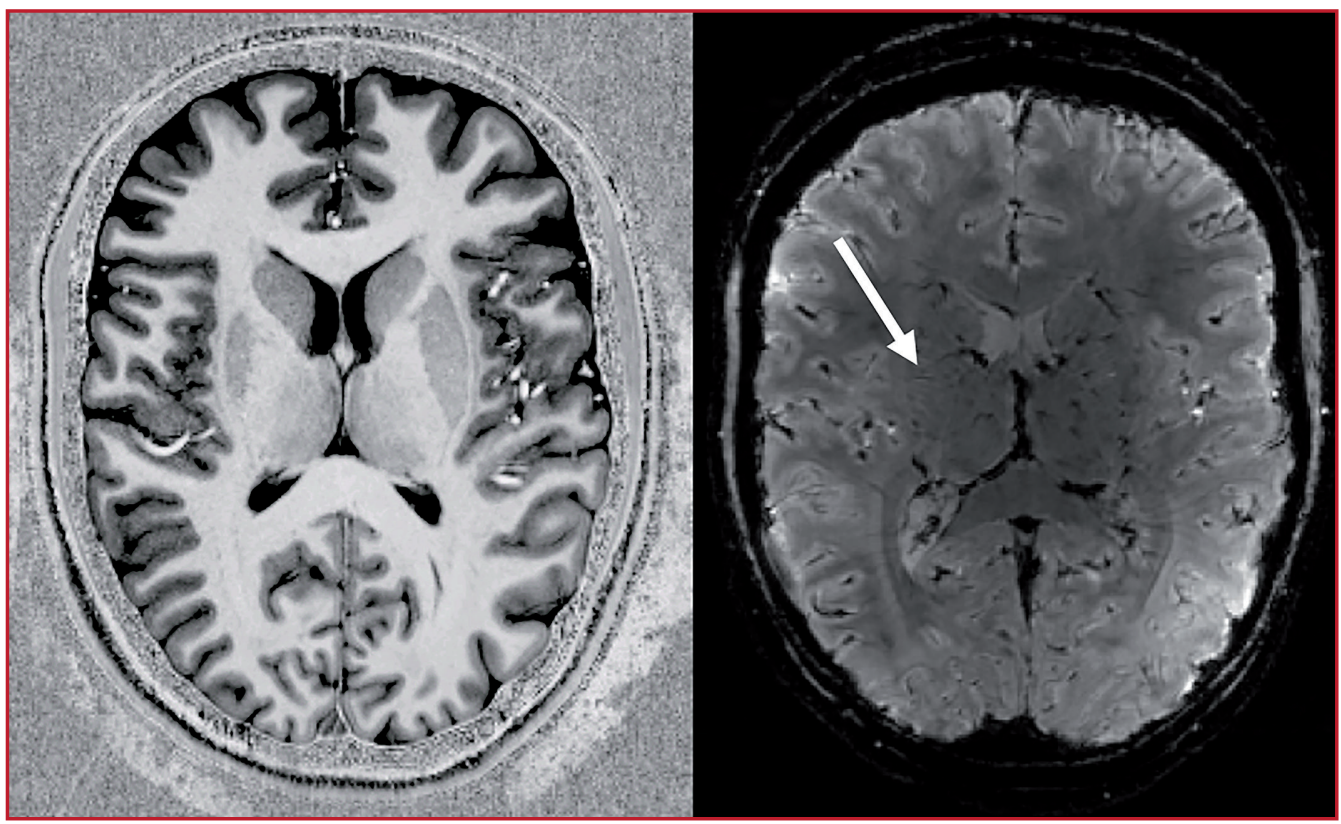

Fig. 3 Axial slices of 7TMRI T1-weighted image from MP2RAGE (a), and T2*weighted image from GRE (b) of a healthy volunteer. White arrow exemplifies the fine vascularisation detail that is observed 
T2-weighted imaging using standard Turbo Spin Echo sequences becomes increasingly difficult at UHF due to relatively high SAR induced by multiple 180 degree refocussing pulses ${ }^{25}$. For this reason, a technique involving the use of a tailored RF flip angle train was used (T2-SPACE) to obtain $\mathrm{T}_{2}$-like contrast ${ }^{26-28}$. This same technique was also used as the basis for a FLAIR-type contrast (SPACE-FLAIR), where an inversion pulse, with inversion time of $2330 \mathrm{~ms}$, was added to null fluid signals. For both T2-weighted sequences, a Works-In-Progress package [WIP 692] from Siemens was used and parameters were optimised to obtain excellent contrast, with reference to standard clinical imaging. All images were reconstructed with and without manufacturer-developed correction procedures to restore gradient uniformity.

\section{Image transfer and processing workflow}

Since MAASTRO cLINIC and Scannexus each operate independently from the Maastricht University Medical Centre (MUMC), a dedicated inter-institutional imaging workflow was developed to be able to collaboratively share the 7T MR images acquired at Scannexus with the department of Neurosurgery at MUMC and Radiation Oncology department at MAASTRO CLINIC. Our solution comprised a cloud-based medical image sharing environment (Quentry, version 3.0, Brainlab AG, Feldkirchen, Germany) to upload the 7T MR images into the neurosurgical cranial navigation software (iPlan Net, version 3.6, Brainlab AG, Feldkirchen, Germany) that will be used to obtain the biopsy specimen for the future clinical study. The MiTools software package was used to generate quantitative $T_{2}{ }^{*}$-maps from the multi-echo GRE images (http://od1n. sourceforge.net/). The 7T MRI scans were imported directly as DICOM files into the radiation treatment planning system (Eclipse version 11, Varian, Palo Alto, United States of America) from the Scannexus facility.

\section{Image quality assessment}

\section{Qualitative image analysis}

During a multi-disciplinary session, a physicist, neuro-radiologist and radiation oncologist visually verified that the MR image quality was warranted after acquisition, transfer and processing of the images. Image quality was assessed by evaluating the images of all three volunteers on the MR scanner console and within the neurosurgical navigation and RTP software. The MP2RAGE, T2-SPACE and SPACE-FLAIR sequences were visually evaluated by an experienced neuro-radiologist for SNR, visualisation of the cerebral lobes, depiction of grey-white matter, basal ganglia, ventricles and CSF around the brain. Each of these factors was classified on a scale of $1-4$ (1. Excellent, 2. Good, 3. Marginal, but still diagnostic, 4. Non-diagnostic). The GRE images were not assessed as these images were post-processed in order to obtain quantitative $\mathrm{T}_{2}{ }^{*}$-images. 


\section{Quantitative image analysis - geometrical image distortion}

To assess the geometrical reliability of the 7T MR images two clinically-relevant pulsesequences (GRE and MP2RAGE) were used to acquire images of the phantom, both with and without automatic geometric distortion correction. Unfortunately, it was found that the distortion correction processing was not compatible with the multiecho data, causing severe artefacts. For this reason, only the data from the MP2RAGE is presented. We aim to resolve this incompatibility in future clinical studies. The GRE and MP2RAGE sequences were selected to assess sequence-related geometrical image distortions as they will be primarily used for GTV delineation in the clinical study.

Next, the 3D coordinates of all 436 grid-intersection points were acquired after manual processing of the respective CT and MR images in Eclipse. The coordinates of the reference points were deduced from the phantom's known geometry.

Two methods were used to measure the geometrical distortion at different levels of sophistication. The first method was used to assess the overall image distortion using an in-house developed MATLAB script (R2014b, MathWorks Inc, Natick, USA). All unique pairs of grid-intersection point coordinates were generated from which subsequently the Euclidean distance was determined, and absolute differences in distance were calculated between the reference (i.e., phantom-based) and measured (i.e., image-based) distances. In a second method, Euclidean distances were measured between a fixed reference point located at the magnetic field isocenter, and all other grid-intersection points in the image, and compared to corresponding distances within the reference frame. Hence, a measure of the geometrical dispersion relative to the magnetic field isocenter was obtained. Both methods were applied to the CT and MRI scans. The overall geometric distortion was quantified by the global mean absolute deviation $\left(M A D_{\text {global }}\right)$ and its standard deviation (SD), while geometrical dispersion relative to the isocenter was quantified by local mean absolute deviation $\left(M A D_{\text {local }}\right)$ and its SD:

$$
\begin{aligned}
& \text { a. } \quad M A D_{\text {global }}=\frac{1}{i} \sum_{i}\left|D_{\text {ref }}-D_{m}\right|_{i} \\
& \text { b. } \quad M A D_{\text {local }}=\frac{1}{i} \sum_{i}\left|D_{\text {ref }}-D_{\text {isoc }}\right|_{i}
\end{aligned}
$$

where $D_{\text {ref }}$ represents the Euclidian distance between reference points, $D_{m}$ is the measured distance between image-based grid points, and $D_{\text {isoc }}$ is the measured distance between the magnetic field isocenter and surrounding grid points. For each measurement within a cluster of unique distances $i$, the MAD is then calculated as the average of the absolute difference in Euclidian distances. 
Given the MAD and its standard deviation (SD), a 95\% confidence interval $(\Delta)$ was calculated as:

$$
\Delta= \pm(M A D+2 S D)
$$

Based on this confidence interval, a maximum acceptable tolerance level of $2.0 \mathrm{~mm}$ was defined. When MAD $>1.0 \mathrm{~mm}$ and $|\Delta|>2.0 \mathrm{~mm}$, the level of geometric deviation between the measured and reference dimensions were considered unacceptable. 


\section{Results}

\section{Image acquisition}

The duration of the scanning sessions for the volunteers was 50 minutes. All three volunteers reported slight vestibular effects while being moved in and out of the magnetic field. One of the volunteers reported twitching of the nose tip during the scanning procedure. No other clinically relevant sensory effects were reported. The duration of the scanning session for the phantom was up to 150 minutes as it was scanned with the same sequences as the volunteers in three orthogonal planes (axial, sagittal, coronal).

\section{Image quality}

\section{Qualitative image analysis}

Images acquired with MP2RAGE (figure 3a), T2-SPACE and SPACE-FLAIR sequences all showed good to excellent SNR and visualization of the frontal and parietal lobes. Furthermore, there was a good to excellent reproduction of the ventricles and CSF surrounding the brain. All sequences showed marginal to good SNR and visualization of the cerebellum. The reproduction of CSF around the cerebellum was good, except for on T2-SPACE where it was judged to be non-diagnostic to marginal. All sequences demonstrated non-diagnostic to marginal image quality for SNR, visualization of the cerebral lobes, depiction of grey-white matter and ventricles and depiction of CSF around the brain at the frontobasal and temporal lobes. This decrease in image quality was primarily caused by a decrease in signal and susceptibility artefacts near the skull base (figure 4). The reproduction of the basal ganglia was good to excellent on T2-SPACE and MP2RAGE, respectively, but was considered marginal in one of the volunteers on SPACE-FLAIR due to flow-artefacts. The visualization of the cerebral vessels was good to excellent on both T2-SPACE and MP2RAGE and considered marginal to good on the SPACE-FLAIR. There was a signal inhomogeneity present in the T2-SPACE and SPACE- FLAIR images in both the medial-lateral and anteriorposterior direction. Moreover, the T2-SPACE showed a signal drop at both the skull base and the temporal lobes. Ghosting artefacts were anteriorly and posteriorly present in the T2-SPACE and SPACE-FLAIR. These may be the result of the relatively high amount of image acceleration used ( $2 \times 2$ GRAPPA) and it may be possible to reduce these artefacts through enhanced image reconstruction. In addition flowartefacts were observed near major intra-cranial vessels such as the carotid and basillary arteries. Image transfer and processing did not visually degrade the image quality. 


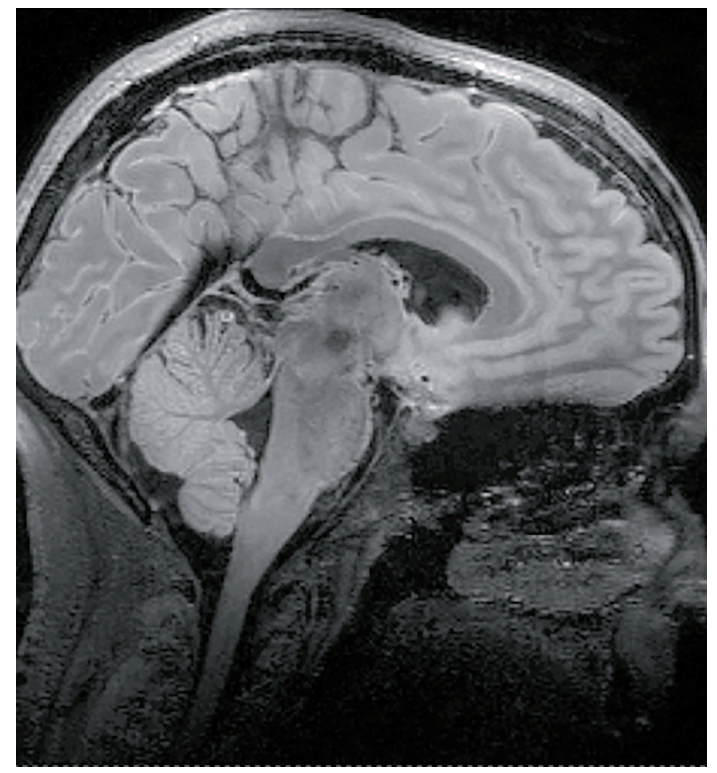

Fig. 4 Sagittal 7T MRI SPACE-FLAIR image of a healthy volunteer, showing increased geometrical distortion near the skull base, the ventral part of the temporal cortices, and the orbito-frontal cortex

\section{Quantitative image analysis - geometrical image distortion}

\section{Global distortion method}

Based on the 436 reference points of the 3D grid, 95266 unique distances between grid-intersection points were calculated and paired into 100 unique distance clusters, ranging from 15 to $164.32 \mathrm{~mm}$. The corresponding imagebased distances were paired into the same clusters for calculating $M A D_{\text {global. }}$ Figure 5 presents the absolute difference between phantom- and image-based distances within each cluster, as well as the $M A D_{\text {global }}$ of that cluster. The global geometric distortion within the MR-images is more pronounced than in CT images. However, $C T$ is not completely free of distortion errors with average $M A D_{\text {global }}$ of 0.20 $\mathrm{mm}(\mathrm{SD} \pm 0.05 \mathrm{~mm}$ ) (fig. 5a). The GRE and MP2RAGE sequences present MAD global ranges of $0.31-1.35 \mathrm{~mm}$ and $0.38-1.62 \mathrm{~mm}$, respectively in distortion-uncorrected images (fig. 5b, 5c). Furthermore, the measured differences were less consistent in MP2RAGE images with various outliers above the $95 \%$ confidence interval $(\Delta=$ $1.29 \mathrm{~mm}$ ), mostly at small intergrid distances. In the distortion-corrected MP2RAGE images, relatively similar patterns of MADglobal could be noted (fig. $5 \mathrm{~d}$ ) with values ranging from $0.34-1.91 \mathrm{~mm}(\Delta=1.68 \mathrm{~mm})$. Table 3 provides a clear overview of MAD ranges, average MAD and MAD confidence intervals per selected image. 

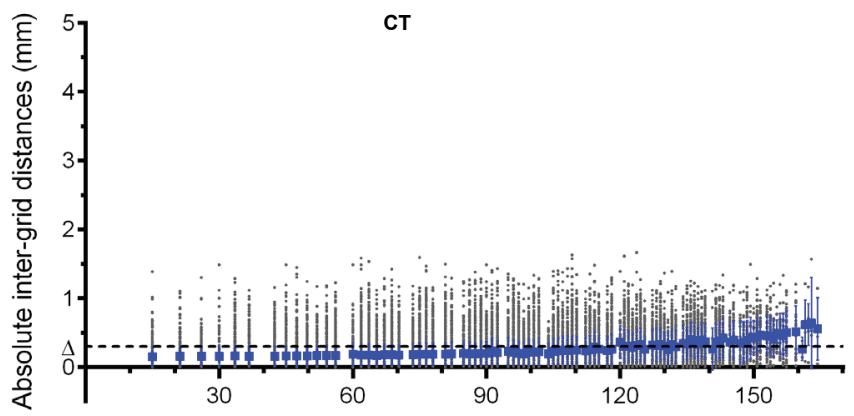

- MAD Global

Cluster distances $(\mathrm{mm})$

GRE
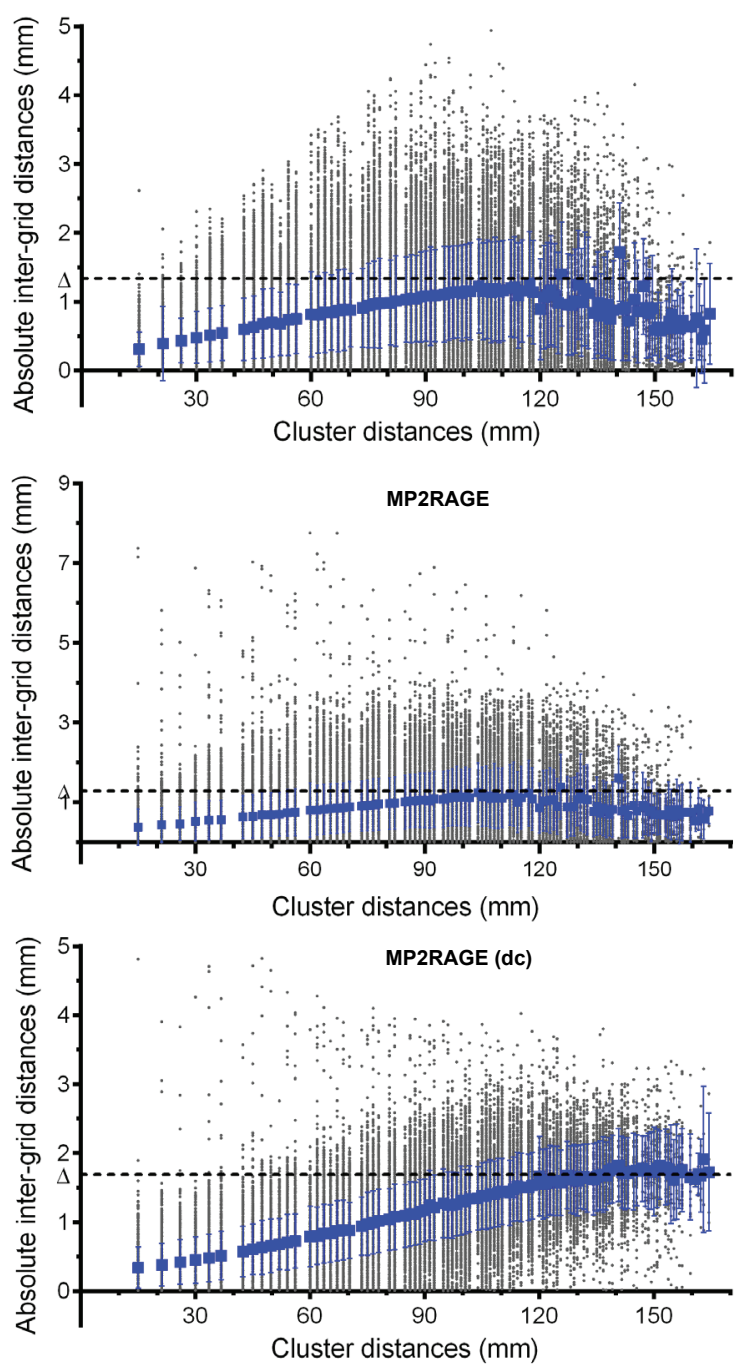

Fig. 5 Absolute inter-grid distances (greydot) measured within CT(a), distortion-uncorrected GRE images (b), distortion-uncorrected MP2RAGE images (c), and distortion-corrected MP2RAGE (d) relative to the possible cluster distances in $\mathrm{mm}$. The overall geometric distortion was quantified by MADglobal (blue square) and its standard deviation. The $95 \%$ confidence interval $(\Delta)$ is shown as the dotted line 


\section{Local distortion method}

Figure 6 shows the geometrical dispersion relative to the isocenter $\left(M A D_{\text {local }}\right)$ and illustrates modality-and sequence-dependent distortion effects and the quality of the MR-distortion correction methods. In CT-images, $M \mathrm{D}_{\text {local }}$ ranges from 0.06-0.27 $\mathrm{mm}$ indicating small spatial deviations. In the distortion-uncorrected 7T MR images, $M A D_{\text {local }}$ ranges from $0.28-1.31 \mathrm{~mm}(\Delta=0.99 \mathrm{~mm})$ in GRE images and $0.26-1.46$ $\mathrm{mm}(\Delta=1.04 \mathrm{~mm})$ in MP2RAGE images. In the distortion-corrected MR-images, $M A D_{\text {local }}$ ranges from $0.22-1.82 \mathrm{~mm}(\Delta=1.56 \mathrm{~mm})$ in MP2RAGE images. The value for $M A D_{\text {local }}$ is observed with increasing distance from the magnetic field isocenter, with a maximum of $1.82 \mathrm{~mm}$ near the edges of the phantom. At equal distance from the isocenter, geometric distortion was found to be anisotropic. For distortion-corrected MP2RAGE images major spatial displacement could be noted in the superior-inferior (SI) direction (e.g., $0.82 \mathrm{~mm}$ at $45 \mathrm{~mm}$ isocenter distance).

Based on the average MAD-values for CT and 7T MRI, both modalities present tolerable levels of geometrical image distortion with average $M A D \leq 1.0 \mathrm{~mm}$ and $\Delta$ $\leq 2.0 \mathrm{~mm}$ (table 2 and 3). However, $M A D_{\text {global }}$ and $M A D_{\text {local }}$ of larger cluster distances often exceed this tolerance limit. For corrected MP2RAGE images, $M_{A} D_{\text {global }}$ was $>1.0$ $\mathrm{mm}$ for intergrid distances $>77.9 \mathrm{~mm}$. $M A D_{\text {local }}$ measurements in distortion-corrected MP2RAGE images was $<1.0 \mathrm{~mm}$ up until $68.7 \mathrm{~mm}$ from the isocenter.

Table 2 Geometric distortion measures for CT

\begin{tabular}{|c|c|c|c|c|c|c|}
\hline & \multicolumn{3}{|c|}{$\mathrm{MAD}_{\text {global }}(\mathrm{mm})$} & \multicolumn{3}{|c|}{$\mathrm{MAD}_{\text {Local }}(\mathrm{mm})$} \\
\hline & Range & Mean \pm SD & $\Delta$ & Range & Mean \pm SD & $\Delta$ \\
\hline CT & $0.14-0.64$ & $0.20 \pm 0.05$ & 0.30 & $0.06-0.27$ & $0.16 \pm 0.04$ & 0.24 \\
\hline
\end{tabular}

Table 3 Geometric distortion measures for 7TMRI

\begin{tabular}{|c|c|c|c|c|c|c|c|}
\hline \multirow[b]{2}{*}{ Sequence } & \multirow[b]{2}{*}{ DC } & \multicolumn{3}{|c|}{$\mathrm{MAD}_{\text {global }}(\mathrm{mm})$} & \multicolumn{3}{|c|}{$\mathrm{MAD}_{\text {Local }}(\mathrm{mm})$} \\
\hline & & Range & Mean \pm SD & $\Delta$ & Range & Mean \pm SD & $\Delta$ \\
\hline GRE & No & $0.31-1.35$ & $0.88 \pm 0.22$ & 1.33 & $0.28-1.31$ & $0.65 \pm 0.17$ & 0.99 \\
\hline MP2RAGE & No & $0.38-1.62$ & $0.88 \pm 0.21$ & 1.29 & $0.26-1.46$ & $0.64 \pm 0.20$ & 1.04 \\
\hline MP2RAGE & $3 D$ & $0.34-1.91$ & $0.98 \pm 0.35$ & 1.68 & $0.22-1.82$ & $0.85 \pm 0.36$ & 1.56 \\
\hline
\end{tabular}



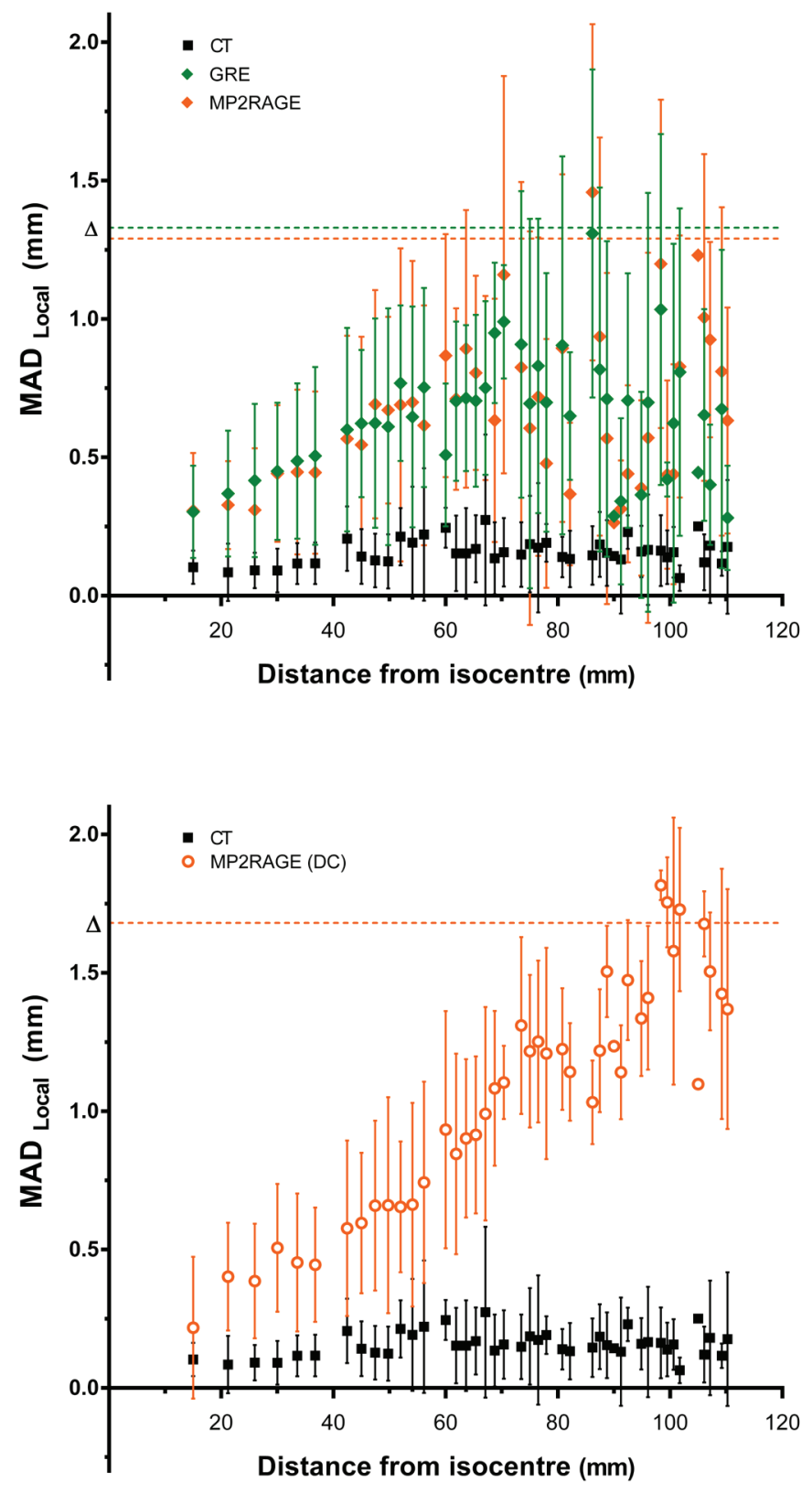

Fig. 6 Geometric dispersion relative to the magnetic field isocenter, quantified by $M A D_{\text {local }}$ and its standard deviation within CT (black square) and distortion-uncorrected $7 T M R$ images (a) acquired with GRE (green diamond) and MP2RAGE (orange diamond) pulse sequences. Measured $M A D_{\text {local }}$ values for CT and distortion-corrected $7 T$ MR images (b) acquired with MP2RAGE (orange circle) pulse sequence are shown relative to the distance 


\section{Discussion}

This paper reports on the preparatory work for our clinical study to integrate 7T MR images into neurosurgical navigation and RTP systems. It includes a pilot study with healthy volunteers and a phantom study. The volunteers in our pilot study reported limited side effects of the 7T MRI. Similar to other studies on UHF MRI, mainly transient vestibular effects were noted 29,30 . In our study these effects were only present during a change in table position. The cause of transient vertigo and dizziness is not yet fully understood, but magnetic stimulation of the ear's labyrinth has been suggested ${ }^{31}$.

There are currently still a few shortcomings in image quality that need to be resolved before clinical implementation of UHF MRI. Although image quality in our study was good to excellent in the frontal and parietal lobes, all sequences demonstrated a nondiagnostic to marginal image quality for all evaluated parameters at the frontobasal and temporal lobes. This is a well-known issue for UHF-MRI and is generally caused by a decrease in signal and susceptibility artefacts near the skull base. As a consequence, patients with tumours near the skull base will be excluded from our clinical trial.

Many researchers have been working on improving the inversion contrast in MPRAGE, particularly trying to reduce the sensitivity to off-resonance and RF inhomogeneity effects $^{19}$. RF transmit technology is a very active research field within UHF MRI, and better transmitter designs may become available. The application of dielectric pads improves the transmit field homogeneity, which in our case ensured obtaining sufficient signal from the temporal regions ${ }^{15,32}$. Concurrently, the application of dielectric pads also affects the receive B1 field and can lead to signal increases in their immediate vicinity. Image intensity inhomogeneities of this kind are also typical for multi-channel RF receive coils. They can be circumvented by image postprocessing techniques such as division by an image with the appropriate weighting or by model fitting of at least two images differing by a single parameter ${ }^{24}$. The latter approach is utilized when generating the $\mathrm{T}_{2}{ }^{*}$ maps from the multi-echo GRE data and T1 maps from the MP2RAGE data, whereas the former approach is used to obtain the MP2RAGE T1-weighted images. It is worth mentioning that the inversion pulse of the MP2RAGE sequence has been specifically designed to deliver homogeneous T1-contrast across the whole brain despite B1-inhomogeneities ${ }^{22}$.

Yet further gains may be possible through the use of parallel transmit (pTx) technology, where 8 or 16 separate elements may be combined to produce a more uniform or targeted excitation. However, the use of such systems involves significant additional experimental setup (e.g., more complicated RF coils and pre-scans to determine per-subject transmit patterns, etc.), which may limit the applicability to patients. Additionally, SAR considerations are significantly more complicated in a pTx setup, as different excitation patterns will produce different SAR values within the subject. While considerable progress has been made, there is still significant work required to implement this in clinical studies. 
The phantom we used provides a realistic anthropomorphic scenario for CT and MRI to assess spatial distortion in 7T MRI for clinical use. Although there is no standard procedure for assessing spatial distortion in 7T MRI, AAPM and the Institute of Physics and Engineering in Medicine recommend to measure the distance between two selected points in the MR-image and to calculate the deviation from the known distance ${ }^{33,34}$. Based on these recommendations we assessed the geometrical image distortion using two different methods, $M A D_{\text {global }}$ and $M A D_{\text {local }}$. We selected two variable points in the image for $M A D_{\text {global }}$ assessment and selected one variable and one fixed point in the image (e.g., magnetic field isocenter) for $M A D_{\text {local }}$ assessment ${ }^{35,36}$.

The assessment of geometrical distortion in our study is similar to the study by Cho et al. investigating the visualisation of targets for deep brain stimulation in Parkinson disease $^{13}$. However, in the present study an updated version of the CIRS phantom was used. Furthermore, we did not consider the CT as a reference standard and therefore did not co-register the CT to the MRI scan. Instead, the known geometry of the phantom was considered the reference.

Although no geometrical distortion was expected, we do report minimal distortion from the CT images. This is probably caused by measurement uncertainties from manually assessing the grid coordinates and was not found to be correlated to inter-grid distances or distances from the isocenter. For MRI however, overall geometric distortion increases exponentially with increasing distances between grid-intersection points. In uncorrected GRE and MP2RAGE images, a plateau-stage starts to manifest at $58 \mathrm{~mm}$ from the isocenter. Presumably, this plateau-stage could be attributed to non-linearity of the gradient fields. Near the edges of the image, the assumed linear relationship between spin position and precession frequency in the local field is false, leading to a compression of the image. In distortioncorrected images, gradient uniformity was restored and as a result, the image was decompressed to its correct dimensions. Consequently, MP2RAGE sequences show a more linear increase of spatial deviation and higher $M A D_{\text {local }}$ maxima are measured (table 3). The larger range of MAD-values is related to the increase in measurable distance between grid-intersection points and the residual system-related geometrical distortion factors, such as eddy currents and $\mathrm{B}_{0}$ inhomogeneity, which were not corrected for. Others have used a similar method as applied in this study to estimate the reliability of stereotactic coordinates on $1.5 \mathrm{~T}$ and $3 \mathrm{~T} \mathrm{MR}$ images ${ }^{37}$. They reported a range of mean errors of $0.30-1.20 \mathrm{~mm}$ and $0.43-1.78 \mathrm{~mm}$ in T1 $\mathrm{w}$-images at $1.5 \mathrm{~T}$ and $3 \mathrm{~T}$, respectively. For T2w-images, they have shown ranges of $0.29-0.58$ $\mathrm{mm}$ and $0.31-1.85 \mathrm{~mm}$, respectively.

Geometric distortion can occur to different extents depending on the imaging sequence parameter setting $\mathrm{s}^{38}$. Differences in $M A D_{\text {local }}$ between GRE and MP2RAGE could be attributed to differences in read-out bandwidth ( $r B W)$. With a lower rBW, more geometric distortion could be noted in the frequency-encoding or read-out 
direction. For MP2RAGE, a low rBW was used $(250 \mathrm{~Hz} /$ pixel) for measurements in the $\mathrm{SI}$ read-out direction, while the read-out direction for GRE images was AP. To minimise this type of geometric distortion, a larger rBW could be selected, however this in turn implies a loss in SNR. Increasing slice thickness could compensate for this loss in SNR, but is undesirable in clinical practise as resolution decreases.

Based on figure 5 and 6 , we conclude that geometric distortion is anisotropic. When measuring local displacement at equal distances from the magnetic field isocenter, the geometric distortion correlates with the frequency-encoding direction. However, the exact quantification of geometric distortion anisotropy was not investigated and will be analysed in future work. When interpreting $M A D_{\text {global }}$ and $M A D_{\text {local }}$ it is important to note that the phantom used in this study describes the dimensions of a realistic human head. This implies that larger measurable distances from the isocenter could be measured in AP and SI direction than are possible in LR direction, leading to a possible misinterpretation of $M A D_{\text {global }}$ and $M A D_{\text {local }}$ and their respective SD.

Geometrical distortion, image registration, and delineation inaccuracies should all be taken into account while establishing the PTV margin for the clinical application of the 7T MRI ${ }^{39}$. Although a larger PTV margin might be less relevant for RTP in GBM in which an isotropic CTV margin of $2-3 \mathrm{~cm}$ is added. An increase in PTV margin is highly detrimental for stereotactic radiosurgery, in which a very high radiation dose is given to a small volume of the brain. Manufacturer's distortion correction routines (i.e., gradient non-linearity correction and automated shimming) were applied to decrease geometric inaccuracies. However, Wang et al. showed that despite these measures, distortion can still be significantly present in UHF MR images ${ }^{40}$. As confirmed by our measurements (fig. 5), the spatial deformation in geometrically corrected MR images is non-negligible for RTP purposes, especially for tumours located far from the magnetic field isocenter. This finding corresponds to the results obtained by other studies using a different phantom ${ }^{12,13}$. Current use of MR images in radiotherapy planning requires co-registration to $\mathrm{CT}$ images as information of electron density is needed for dose calculation. Although CT shows negligible geometric distortion, co-registration of $C T$ and MR images introduces registration errors ${ }^{41}$. MRI-only based treatment planning could provide an alternative to errors introduced through registration, but requires thorough investigation before clinical implementation ${ }^{42}$.

Any technical and logistic challenges we encountered in the image transfer and processing workflow were primarily caused by the different image acquisition and processing platforms used at the different institutions and departments involved in this study, e.g. 7T MRI scanner at Scannexus, neurosurgical and RTP software. Not all image data formats (e.g. NIfTi, xBrain) were compatible with these software systems. Fortunately, we could solve these problems by converting all image datasets into 
DICOM format and by using a cloud-based environment to collaboratively share the images. However, transfer and conversion of images could carry an inherent risk of data degradation and should be checked prior to clinical use.

The present study has several limitations and some factors that need to be considered when attempting to setup a similar study. First, the number of volunteers included in our pilot study was small. Moreover, sequence optimization was limited by the increase in SAR and scan duration and leaves room for future improvement. Second, unlike a human head, the anthropomorphic skull phantom did not contain any air cavities (i.e., sinuses) and only allowed assessment of the system-related geometrical distortion. Third, we only investigated geometrical distortion of 7T MRI in comparison to $C T$ and did not yet evaluate lower field strength MR images. Geometrical distortion in relation to $1.5 \mathrm{~T}$ and $3 \mathrm{~T}$ field strengths will be addressed in a future study. Fourth, other hardware related geometrical distortions such as eddy currents and $B_{0}$ inhomogeneity are not corrected and remain present in the images. In general, $B_{0}$ inhomogeneity could be resolved with shimming and field map correction, although these corrections are mostly effective in EPI sequences ${ }^{43,44}$. Finally, the distortion corrected GRE images could not be assessed for geometrical distortion due to severe artefacts, therefore a comparison between distortion corrected pulse sequences was not possible.

Several investigators suggested the presence of microvasculature on UHF MRI may aid in improved identification of WHO tumour grade in GBM as compared to $3 T$ $\mathrm{MRI}^{8,10}$. So far, these findings have not resulted in a systematic investigation of the potential value of 7T MRI for improved neurosurgical navigation and target volume definition in RTP. Therefore, we aim to demonstrate the potential benefit of UHF MRI in identifying tumour infiltration for the delineation of GBM in our upcoming clinical study. This study is expected to open for inclusion in the first quarter of 2016. During the preparatory phase of the current study image quality has significantly improved. However, further improvement of the image quality by reducing susceptibility artefacts in the frontobasal region and in the temporal lobe is required before clinical implementation.

\section{Conclusion}

Although the integration of high quality and geometrically reliable 7T anatomical MR images into neurosurgical navigation and RTP software is technically feasible and safe for the system and pulse sequences studied, image quality needs further improvement before it can be integrated into the clinical workflow. System-related geometrical distortions of the 7T sequences studied are clinically acceptable for image co-registration with $\mathrm{CT}$ prior to radiotherapy and for direct use in neurosurgical procedures. 
1. Stupp R, Hegi ME, Mason WP, et al. Effects of radiotherapy with concomitant and adjuvant temozolomide versus radiotherapy alone on survival in glioblastoma in a randomised phase III study: 5-year analysis of the EORTC-NCIC trial. Lancet Oncol. 2009;10(5):459-466.

2. Claes A, Idema AJ, Wesseling P. Diffuse glioma growth: a guerilla war. Acta neuropathologica. 2007;114(5):443-458.

3. Weller $M$, van den Bent $M$, Hopkins $K$, et al. EANO guideline for the diagnosis and treatment of anaplastic gliomas and glioblastoma. Lancet Oncol. 2014;15(9): e395-403.

4. Chang EL, Akyurek S, Avalos T, et al. Evaluation of peritumoral edema in the delineation of radiotherapy clinical target volumes for glioblastoma. Int J Radiat Oncol Biol Phys. 2007;68(1):144-150.

5. Oppitz U, Maessen D, Zunterer H, Richter S, Flentje M. 3D-recurrence-patterns of glioblastomas after CT-planned postoperative irradiation. Radiother Oncol. 1999;53(1):53-57.

6. Kuroiwa T, Ueki M, Chen Q, Suemasu H, Taniguchi I, Okeda R. Biomechanical characteristics of brain edema: the difference between vasogenic-type and cytotoxic-type edema. Acta Neurochir Suppl (Wien). 1994;60:158-161.

7. Louis DN, International Agency for Research on Cancer, World Health Organization. WHO classification of tumours of the central nervous system. 4th ed. Lyon: International Agency for Research on Cancer, 2007.; 2007.

8. Moenninghoff $C$, Maderwald S, Theysohn JM, et al. Imaging of adult astrocytic brain tumours with 7 T MRI: preliminary results. Eur Radiol. 2010;20(3):704-713.

9. Christoforidis GA, Grecula JC, Newton HB, et al. Visualization of microvascularity in glioblastoma multiforme with 8-T high-spatial-resolution MR imaging. AJNR Am J Neuroradiol. 2002;23(9):1553-1556.

10. Christoforidis GA, Yang M, Abduljalil A, et al. "Tumoral pseudoblush" identified within gliomas at high-spatial-resolution ultrahigh-field-strength gradient-echo MR imaging corresponds to microvascularity at stereotactic biopsy. Radiology. 2012;264(1):210-217.

11. Lupo JM, Li Y, Hess CP, Nelson SJ. Advances in ultra-high field MRI for the clinical management of patients with brain tumors. Curr Opin Neurol. 2011;24(6):605615.

12. Dammann $\mathrm{P}$, Kraff $\mathrm{O}$, Wrede $\mathrm{KH}$, et al. Evaluation of hardware-related geometrical distortion in structural MRI at 7 Tesla for image-guided applications in neurosurgery. Acad Radiol. 2011;18(7):910-916.

13. Cho $\mathrm{ZH}, \mathrm{Min} \mathrm{HK}$, Oh $\mathrm{SH}$, et al. Direct visualization of deep brain stimulation targets in Parkinson disease with the use of 7-tesla magnetic resonance imaging. J Neurosurg. 2010;113(3):639-647.

14. Duchin Y, Abosch A, Yacoub E, Sapiro G, Harel N. Feasibility of using ultra-high field (7 T) MRI for clinical surgical targeting. PLoS One. 2012;7(5):e37328. 
15. Teeuwisse WM, Brink WM, Haines KN, Webb AG. Simulations of high permittivity materials for $7 \mathrm{~T}$ neuroimaging and evaluation of a new barium titanate-based dielectric. Magnetic resonance in medicine. 2012;67(4):912-918.

16. Schenck JF. The role of magnetic susceptibility in magnetic resonance imaging: MRI magnetic compatibility of the first and second kinds. Medical physics. 1996;23(6):815-850.

17. Marques JP, Kober T, Krueger G, van der Zwaag W, Van de Moortele PF, Gruetter R. MP2RAGE, a self bias-field corrected sequence for improved segmentation and T1-mapping at high field. Neuroimage. 2010;49(2):1271-1281.

18. Mugler JP, 3rd, Brookeman JR. Three-dimensional magnetization-prepared rapid gradient-echo imaging (3D MP RAGE). Magnetic resonance in medicine. 1990;15(1):152-157.

19. Wrede $\mathrm{KH}$, Johst $\mathrm{S}$, Dammann $\mathrm{P}$, et al. Caudal image contrast inversion in MPRAGE at 7 Tesla: problem and solution. Acad Radiol. 2012;19(2):172-178.

20. Marques JP, Gruetter R. New developments and applications of the MP2RAGE sequence--focusing the contrast and high spatial resolution R1 mapping. PLoS One. 2013;8(7):e69294.

21. O'Brien KR, Kober $T$, Hagmann $P$, et al. Robust T1-weighted structural brain imaging and morphometry at 7T using MP2RAGE. PLoS One. 2014;9(6):e99676.

22. Hurley AC, Al-Radaideh A, Bai L, et al. Tailored RF pulse for magnetization inversion at ultrahigh field. Magnetic resonance in medicine. 2010;63(1):51-58.

23. Okubo G, OkadaT,Yamamoto A, etal.MP2RAGE for deep gray matter measurement of the brain: A comparative study with MPRAGE. J Magn Reson Imaging. 2015.

24. Van de Moortele PF, Auerbach EJ, Olman C, Yacoub E, Ugurbil K, Moeller S. T1 weighted brain images at 7 Tesla unbiased for Proton Density, T2* contrast and $\mathrm{RF}$ coil receive $\mathrm{B} 1$ sensitivity with simultaneous vessel visualization. Neuroimage. 2009;46(2):432-446.

25. Hennig J, Nauerth A, Friedburg H. RARE imaging: a fast imaging method for clinical MR. Magnetic resonance in medicine. 1986;3(6):823-833.

26. Mugler John P. KRB, Brookeman James R. . Three-dimensional T2-weighted imaging of the brain using very long spin-echo trains. . Proc 8th ISMRM 2000.

27. Mugler John P. WLL, Brookeman James R. . T2-weighted 3D spin-echo train imaging of the brain at 3 Tesla: reduced power deposition using low flip-angle refocusing RF pulses. . Proc 8th ISMRM. 2001.

28. Busse RF, Hariharan $\mathrm{H}, \mathrm{Vu} \mathrm{A}$, Brittain JH. Fast spin echo sequences with very long echo trains: design of variable refocusing flip angle schedules and generation of clinical T2 contrast. Magnetic resonance in medicine. 2006;55(5):1030-1037.

29. Schaap K, Christopher-de Vries Y, Mason CK, de Vocht F, Portengen L, Kromhout $\mathrm{H}$. Occupational exposure of healthcare and research staff to static magnetic stray fields from 1.5-7 Tesla MRI scanners is associated with reporting of transient symptoms. Occupational and environmental medicine. 2014;71(6):423-429.

30. Heilmaier C, Theysohn JM, Maderwald S, Kraff O, Ladd ME, Ladd SC. A large-scale study on subjective perception of discomfort during 7 and 1.5T MRI examinations. Bioelectromagnetics. 2011;32(8):610-619. 
31. Ward BK, Roberts DC, Della Santina CC, Carey JP, Zee DS. Vestibular stimulation by magnetic fields. Annals of the New York Academy of Sciences. 2015;1343:69-79.

32. Brink WM, van der Jagt AM, Versluis MJ, Verbist BM, Webb AG. High permittivity dielectric pads improve high spatial resolution magnetic resonance imaging of the inner ear at 7 T. Invest Radiol. 2014;49(5):271-277.

33. Price RR, Axel L, Morgan T, et al. Quality assurance methods and phantoms for magnetic resonance imaging: report of AAPM nuclear magnetic resonance Task Group No. 1. Medical physics. 1990;17(2):287-295.

34. Lerski RA, Schad LR. The use of reticulated foam in texture test objects for magnetic resonance imaging. Magn Reson Imaging. 1998;16(9):1139-1144.

35. Wang D, Doddrell DM, Cowin G. A novel phantom and method for comprehensive 3-dimensional measurement and correction of geometric distortion in magnetic resonance imaging. Magn Reson Imaging. 2004;22(4):529-542.

36. Roue A, Ferreira IH, Van Dam J, Svensson H, Venselaar JL. The EQUAL-ESTRO audit on geometric reconstruction techniques in brachytherapy. Radiother Oncol. 2006;78(1):78-83.

37. Kim HY, Lee SI, Jin SJ, Jin SC, Kim JS, Jeon KD. Reliability of stereotactic coordinates of 1.5-tesla and 3-tesla MRI in radiosurgery and functional neurosurgery. Journal of Korean Neurosurgical Society. 2014;55(3):136-141.

38. Walker A, Liney G, Metcalfe P, Holloway L. MRI distortion: considerations for MRI based radiotherapy treatment planning. Australasian physical \& engineering sciences in medicine / supported by the Australasian College of Physical Scientists in Medicine and the Australasian Association of Physical Sciences in Medicine. 2014;37(1):103-113.

39. van Herk M. Errors and margins in radiotherapy. Seminars in radiation oncology. 2004;14(1):52-64.

40. Wang D, Strugnell W, Cowin G, Doddrell DM, Slaughter R. Geometric distortion in clinical MRI systems Part II: correction using a 3D phantom. Magn Reson Imaging. 2004;22(9):1223-1232.

41. Baldwin LN, Wachowicz K, Thomas SD, Rivest R, Fallone BG. Characterization, prediction, and correction of geometric distortion in $3 \mathrm{~T}$ MR images. Medical physics. 2007;34(2):388-399.

42. Schmidt MA, Payne GS. Radiotherapy planning using MRI. Physics in medicine and biology. 2015;60(22):R323-361.

43. Chang HC, Chuang TC, Lin YR, Wang FN, Huang TY, Chung HW. Correction of geometric distortion in Propeller echo planar imaging using a modified reversed gradient approach. Quantitative imaging in medicine and surgery. 2013;3(2):73-81.

44. Wang FN, Huang TY, Lin FH, et al. PROPELLER EPI: an MRI technique suitable for diffusion tensor imaging at high field strength with reduced geometric distortions. Magnetic resonance in medicine. 2005;54(5):1232-1240. 
Chapter 5 - Characterizing geometrical accuracy in clinically optimized 7T and 3T MR images for high-precision radiation treatment of brain tumours

Jurgen Peerlings, Inge Compter, Fiere Janssen, Christopher J. Wiggins, Alida A. Postma, Felix M. Mottaghy, Philippe Lambin, Aswin L. Hoffmann

Physics and Imaging in Radiation Oncology. 2019;9:35-42. 


\section{Abstract}

\section{Background and Purpose}

To assess whether the spatial accuracy in 7-Tesla (7T) MR images, previously optimised for anatomical imaging, is sufficient for clinically-acceptable radiation treatment planning(RTP) in neuro-oncology in respect to standard clinical imaging-modalities. Materials and Methods

System- and phantom-related geometrical distortion(GD) was quantified on clinically-relevant MR-sequences at 7T and 3T, and on CT-images using a dedicated anthropomorphic head-phantom incorporating a 3D grid-structure, creating 436 points-of-interest. Global GD was assessed by mean absolute deviation ( $\mathrm{MAD}_{\text {global }}$ ). Local GD relative to the magnetic isocentre was assessed by $M A D_{\text {local }}$ Using $3 D$ displacement-vectors of individual points-of-interest, GD maps were created. For clinically acceptable radiotherapy, 7T-images need to meet the criteria for accurate dose delivery $(\mathrm{GD}<1 \mathrm{~mm})$ and present comparable $\mathrm{GD}$ as tolerated in clinicallystandard 3T-MR/CT-based RTP.

\section{Results}

$M A D_{\text {global }}$ in 7T- and 3T-images ranged from $0.3-2.2 \mathrm{~mm}$ and $0.2-0.8 \mathrm{~mm}$, respectively. $M A D_{\text {local }}$ increased with increasing distance from the isocentre, showed an anisotropic distribution, and was significantly larger in 7T MR-sequences $\left(M A D_{\text {local }}=0.2-1.2 \mathrm{~mm}\right.$ ) than in $3 T\left(M A D_{\text {local }}=0.1-0.7 \mathrm{~mm}\right)(\mathrm{p}<0.05)$. Significant differences in $\mathrm{GD}$ were detected between 7 T-images $(p<0.001)$. However, maximum $M A D_{\text {local }}$ remained $\leq 1 \mathrm{~mm}$ within $68.7 \mathrm{~mm}$ diameter spherical volume. No significant differences in GD were found between 7T- and 3T-protocols near the isocentre.

\section{Conclusions}

System- and phantom-related GD remained $\leq 1 \mathrm{~mm}$ in central brain regions, suggesting that 7T MR-images could be implemented in radiotherapy with clinicallyacceptable spatial accuracy and equally tolerated GD as in 3T-MR/CT-based RTP. For peripheral regions, GD should be incorporated in safety margins for treatment uncertainties. Moreover, the effects of sequence-related factors on GD needs further investigation to obtain RTP-specific MR protocols. 


\section{Introduction}

In radiation treatment planning (RTP) of brain tumours, magnetic resonance imaging (MRI) at 1.5 or 3 Tesla ( $\mathrm{T}$ ) is currently being used as the standard anatomical imaging modality owing to its superior soft-tissue contrast compared to computed tomography $(\mathrm{CT})^{1}$. Generally, after co-registration of MR and CT images, the former is used for target volume definition and the latter is used for dose calculation, as tissue electron density information is missing in MR-images. However, the current clinical MR-techniques are limited in depicting detailed neurologic malformations such as intracerebral tumour spread ${ }^{2}$. With the prospect of clinically-certified ultrahigh field (UHF-)MRI systems ( $\geq 7 \mathrm{~T}$ ), images with higher signal-to-noise ratio (SNR), higher spatial resolution, and novel contrast mechanisms such as quantitative susceptibility-weighted images will become available, enabling the visualisation of small lesions, basal ganglia, and tumour angiogenesis $\mathrm{s}^{3-5}$. The improved ability in detecting microvasculature (diameter $\sim 100 \mu \mathrm{m}$ ) could play a decisive role in staging primary brain tumours such as glioblastoma (GBM), as tumour angiogenesis is directly associated with tumour grade, and could aid in determining relevant location of dedifferentiated cells for image-guided biopsies ${ }^{6,7}$. In addition, 7T-MRI could aid target volume definition in RTP by visualising microvasculature outside the contrastenhanced tumour and infiltration of GBM cells along white matter tracts ${ }^{7-9}$. During follow-up scans, 7T-MRI has been shown to reveal radiation-induced microbleeds, indicating potential neurocognitive decline and the need to adapt RTP ${ }^{10,11}$.

However, concerns regarding geometrical distortion (GD) with increasing static magnetic field strength (B0) have compromised the integration of UHF-MRI into radiation treatment planning. System-related $G D$ are mainly induced by imperfections in hardware (B0-inhomogeneity, gradient field nonlinearity, and eddy currents), applied radiofrequency (RF) pulse and parameter settings (sequence-dependent GD), while phantom-related GD (chemical shift and susceptibility differences) are related to the object's shape placed in the MR-system ${ }^{12,13}$. To be applicable for highprecision RTP, the spatial accuracy of anatomical images needs to be within $2 \mathrm{~mm}$ for non-stereotactic radiotherapy and within $1 \mathrm{~mm}$ for stereotactic radiotherapy, as is the case for 3T-MRI ${ }^{13-18}$. In fact, profound GD may have significant dosimetric impact in radiotherapy as the precision of beam targeting and dose calculations could be affected $^{19,20}$. For high-precision dose delivery techniques that apply steep 3D dose gradients (intracranial stereotactic radiotherapy or radiosurgery), spatial inaccuracies of $>1 \mathrm{~mm}$ could result in clinically-unacceptable dose variations of more than $15 \%$ that could lead to underdosage of target volume and/or overdosage of surrounding tissue ${ }^{20,21}$. Ultimately, inaccuracy of beam targeting and dose calculation could lead to a different clinical outcome and local tumour control ${ }^{13,18}$. For instance, techniques that apply extremely steep dose gradients, such as stereotactic radiotherapy and radiosurgery, are susceptible to significant dosimetric effects (5-32\% underdosage) caused by even small GD of $1.3 \mathrm{~mm}^{21,22}$. The question therefore arises whether our 
clinical 7T-MRI protocol, optimised for anatomical imaging, can produce images with acceptable spatial accuracy needed for reliable high-precision radiation treatment of brain tumours. Previously several investigators have evaluated geometrical distortion on either CT, 3T- or 7T-MRI, demonstrating a maximum GD of up to several $\mathrm{mm}$ in large cylindrical or cubical phantoms $\mathbf{s}^{14,23-27}$.

This study aims to investigate the clinical applicability of anatomical 7T-MRI in comparison to 3T-MRI and CT for reliable high-precision radiotherapy by evaluating the magnitude and location of hardware-related GD. We hypothesise: 1) acceptable submillimetre spatial accuracy could be achieved in our 7T-MRI protocol and no significant differences in GD are present between different 7T-sequences; 2) the tolerated level of GD in 7T-MRI is not significantly different from system-related GD in a predefined 3T-MRI protocol, clinically used for RTP. To test these hypotheses on both global level (GD throughout the entire volume-of-interest (VOI)) and on local level (GD relative to the distance from the magnetic isocentre), we performed quantitative image analyses using a dedicated anthropomorphic head-phantom in a clinically-realistic set-up. Hence, different sequence parameter settings and distortion correction methods were not explored in this study. 


\section{Materials and methods}

\section{Phantom}

A dedicated anthropomorphic head-phantom (CIRS Model 603A, Computerized Imaging Reference Systems Inc., USA) was used to evaluate system-related GD in 7T-MRI, 3T-MRI, and CT-images (Fig. S.1). The anatomical shape demanded similar procedures used for clinically imaging patients (FOV setting, shimming, robust positioning inside the scanner and head-coil). Furthermore, the centre of the phantom was aligned with the magnetic isocentre.

The phantom was composed of a plastic-based bone substitute and soft-tissue equivalent fluid consisting of a water-based polyacrylamide with added $\mathrm{NiCl} 2$ (Zerdine ${ }^{\oplus}, \mathrm{CIRS}$ ). Inside the phantom, 3-mm thick nylon rods formed a 3D grid with 15 $\mathrm{mm}$ spacing ( $\pm 0.05 \mathrm{~mm}$ manufacturing tolerance), defining a VOI of $15 \times 12 \times 13.5 \mathrm{~cm} 3$ (anterior-posterior $(\mathrm{AP}) \times$ left-right $(\mathrm{LR}) \times$ superior-inferior $(\mathrm{SI}))$. At grid-intersections, 437 measurable points-of-interest (POIs) were identified. Nylon shows similar magnetic susceptibility properties to water $(X<3 \mathrm{ppm})$ and was assumed not to induce such susceptibility artefacts in spin-echo or gradient-echo sequences ${ }^{24,28}$.

\section{Image acquisition}

MR images were acquired on a 7T-MR system (Magnetom 7T, Siemens, DEU) and on a 3T-MR system (Achieva 3T, Philips, NL) using 32-channel head coils from Nova Medical inc. (1Tx/32Rx, Wilmington, USA) and Invivo Corp. (Florida, USA), respectively. The 7T-MR system was equipped with Siemens' $\mathrm{SC} 72$-gradient system $(80 \mathrm{mT} / \mathrm{m}$ peak gradient amplitude, $200 \mathrm{~T} / \mathrm{m} / \mathrm{s}$ maximum slew rate). The 3T-MR system was fitted with Philips' Dual Quasar gradient system ( $80 \mathrm{mT} / \mathrm{m}$ peak gradient amplitude, 100 $\mathrm{mT} / \mathrm{m} / \mathrm{s}$ maximum slew rate). All MR sequences were previously optimised to acquire anatomical images of high image-quality within an acceptable scan duration, and were not altered for this study[9]. The selected 7T-MR sequences included magnetization-prepared rapid gradient-echo (MP2RAGE), T2-sampling perfection with application optimized contrasts using different flip angle evolution (T2-SPACE), T2-SPACE with fluid attenuation inversion recovery (T2-SPACE FLAIR), and multiecho gradient-echo (T1-GRE) ${ }^{9}$. Sequences with equivalent pulse schemes were selected from a predefined clinical 3T-MR protocol used in RTP and included turbo field echo (3D TFE), volume isotropic turbo spin-echo (T2-VISTA) with and without FLAIR, and fast field gradient echo (T1-FFE), respectively (Table S.1). Vendor-installed $3 \mathrm{D}$ distortion correction methods were applied to restore gradient non-linearity and $3 \mathrm{D}$ volume-based second order shimming procedures were applied to improve $\mathrm{B}_{0}$ homogeneity ${ }^{14,29,30}$.

CT images were acquired (SOMATOM Sensation 10, Siemens, Germany) with a slice thickness of $1 \mathrm{~mm}, 306$ slices, $50 \times 50 \mathrm{~cm}^{2} \mathrm{FOV}, 140 \mathrm{kV}$, and $400 \mathrm{mAs}$. 


\section{Methods of analyses}

The known 3D coordinates of all POIs were defined as reference dataset and manually reconstructed in MRI and CT images using Eclipse treatment planning system (Varian Medical Systems, USA). The X-, Y-, and Z-axes defined the LR-, SI-, and AP-directions in both datasets, respectively.

Three methods were used to assess GD at different levels of sophistication using in-house developed MATLAB software (R2014b, MathWorks Inc, USA) (Fig.1). Firstly, mean absolute deviation (MAD) was computed to assess GD on a global level $\left(M A D_{\text {global }}\right)$ throughout the entire $\mathrm{VOI}$, independently of location within the phantom. Secondly, MAD was calculated to assess $G D$ on a local level $\left(M A D_{\text {local }}\right)$ relative to the distance from the magnetic isocentre. MAD was calculated according to:

$$
M A D(i)=\frac{1}{N(i)} \sum_{j=1}^{N(i)}\left|D_{i}(j)-\delta_{i}\right|
$$

where $i=1 \ldots M$ is an index into set $\Delta=\left\{\delta_{1}, \delta_{2}, \ldots, \delta_{M}\right\}$ of $M$ unique distances, $D_{i}(j)$ is the measured Euclidean distance for the $j$-th unique pair of POls having an Euclidean distance in the reference dataset, and represents the number of unique pairs of POls. For $M A D_{\text {global' }} \Delta$ contained all unique distances that could be observed within the entire phantom. For $M A D_{\text {local' }} \Delta$ contained only unique distances that originate from the POI nearest the magnetic isocentre. The mean MAD was averaged over all $M$ unique distances in $\Delta$

The third method of analyses pin-pointed GD by calculating 3D displacement of each individual POI by computing the Euclidean distance directly between the POIcoordinates in the image $\left(X_{m^{\prime}}, Y_{m^{\prime}} Z_{m}\right)$ and the coordinates in the reference datasets $\left(X_{\text {ref }} Y_{\text {ref }} Z_{\text {ref }}\right)$ :

$$
D_{x y z}=\sqrt{\left(X_{m}-X_{r e f}\right)^{2}+\left(Y_{m}-Y_{r e f}\right)^{2}+\left(Z_{m}+Z_{r e f}\right)^{2}}
$$

The resulting 3D displacement vector $\left(D_{x y z}\right)$ indicated relative $G D$ of each individual $\mathrm{POI}$, independently of all other POIs.

The three methods of analyses provided complementary information on the geometrical quality of the images. The $M A D_{\text {global }}$ quantified the level of GD throughout the entire $\mathrm{VOI}$, thereby ignoring information on the absolute and relative locations of the POls. The $M A D_{\text {local }}$ retained spatial information by quantifying the GD with respect to the diameter of a spherical volume (DSV) at the magnetic isocentre. 
To quantify spatial integrity of an entire image, 95\% confidence interval $\left(\left|\mathrm{Cl}_{95}\right|\right)$ was calculated from MAD and standard deviation (SD) as:

$$
\left|C I_{95}\right|=M A D+2 S D
$$

Based on this interval, a maximum acceptability-level of $1 \mathrm{~mm}$ was defined. The level of GD was considered acceptable when MADlocal $\leq 1 \mathrm{~mm}$ (at a specific DSV) and $|\mathrm{Cl} 95| \leq 1 \mathrm{~mm}$.

1a. Measured dataset: image-based

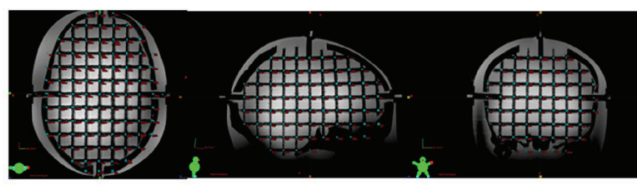

2. Mean Absolute Deviation (MAD)

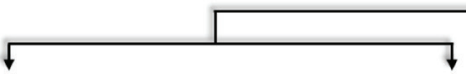

2a. Global MAD*

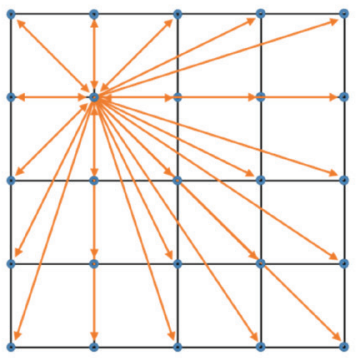

2b. Local MAD

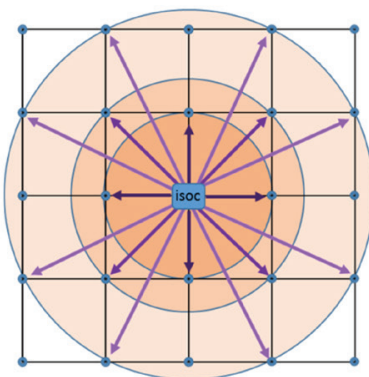

1b. Reference data: phantom-based
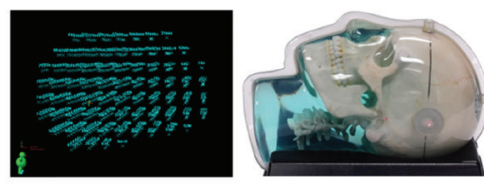

3. Relative deviation

3a. Displacement vector

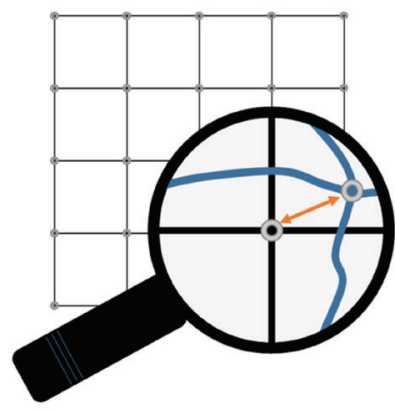

Fig. 1: Overview of 3D data acquisition and analyses. $X Y Z$-coordinates are determined on CT and MR images (1a.), and based on product characteristics of the CIRS' phantom model $603 A$ (1b.). Global MAD (2a.) is based on distances with 2 variable grid-intersection points ( ${ }^{*}$ example given for 1 intersection but applies for all points). Local MAD (2b.) is based on distances between magnetic field isocentre and 1 variable grid-intersection points. Displacements vectors (3a.) are determined between the measured and reference coordinates of each individual data-point and indicate the relative $3 D$ geometrical distortion 


\section{Statistical Analyses}

To assess differences in sequence-dependent $G D, M A D_{\text {global }}$ and $M A D_{\text {local }}$ were analysed between 7T-MRI sequences and between 3T-MRI sequences using a Kruskal-Wallis test. To assess differences in tolerated GD-levels, $M A D_{\text {global }}$ and $M A D_{\text {local }}$ were analysed between equivalent sequence pulse schemes in 7T- and 3T-MRI using a multiple student t-test with Bonferonni-Sidak correction. For $M A D_{\text {local' }}$ DSV at which statistical significance in $M A D_{\text {local }}$ between sequences occurred, was identified by a separate Kruskal-Wallis test for each set of unique distances. All statistical analyses were performed using GraphPad Prism v6.01 (GraphPad, USA). P-values $<0.05$ were considered to indicate statistical significance.

\section{Results}

\section{Global GD}

In total, 95.266 pairs of distances were identified and binned into 100 unique distances, ranging from $15 \mathrm{~mm}$ to $164.3 \mathrm{~mm}$. In $C T$ images, $M A D_{\text {global }}$ ranged from $0.1-0.6 \mathrm{~mm}$ with mean $M A D_{\text {global }}$ of $0.3 \pm 0.1 \mathrm{~mm}$. For $M R$ images, $M A D_{\text {global }}$ ranged from $0.3-2.2 \mathrm{~mm}$ and $0.2-0.8 \mathrm{~mm}$ in 7T- and 3T-MRI, respectively (Table 1, Fig.2). Based on $\left|\mathrm{Cl}_{95}\right|$, only $3 \mathrm{~T}-\mathrm{MR}$ sequences presented $\mathrm{GD}<1 \mathrm{~mm}$ throughout the entire image. No statistical significance in $M A D_{\text {global }}$ were found between 7T-MR images $(p=0.38)$ or 3 T-MR images $(p=0.13)$. However, significant differences in $M A D_{\text {global }}$ were detected between all equivalent sequences on 7T and 3T MR-system $(p<0.001)$.

(Fig. 3.).

Table 1: Global geometrical deformation as measured on $3 T$ and $\left.7 T M R I{ }^{* * *}=P<0.001\right)$

\begin{tabular}{|c|c|c|c|c|c|c|c|c|c|}
\hline \multicolumn{5}{|c|}{$M A D_{\text {Global }}$ (in $\mathrm{mm}$ ) } & \multicolumn{4}{|c|}{ MAD $_{\text {Global }}$ (in $\left.\mathrm{mm}\right)$} & \multirow[b]{2}{*}{$P$ value } \\
\hline $\mathrm{B}_{0}$ & Sequence & Range & Mean $\pm \mathrm{sd}$ & $\left|\mathrm{Cl}_{95}\right|$ & $\mathrm{B}_{0}$ Sequence & Range & Mean $\pm s d$ & $\left|\mathrm{Cl}_{95}\right|$ & \\
\hline \multirow[t]{4}{*}{$7 \mathrm{~T}$} & MP2RAGE & $0.3-1.9$ & $1.0 \pm 0.3$ & 1.7 & 3T 3D TFE & $0.22-0.51$ & $0.27 \pm 0.23$ & 0.7 & $* * *$ \\
\hline & SPACE & $0.3-2.0$ & $1.0 \pm 0.4$ & 1.7 & T2-VISTA & $0.20-0.70$ & $0.26 \pm 0.20$ & 0.7 & $* * *$ \\
\hline & SPACEFLAIR & $0.3-2.2$ & $0.9 \pm 0.3$ & 1.6 & T2-VISTA FLAIR & $0.19-0.62$ & $0.29 \pm 0.23$ & 0.7 & $* * *$ \\
\hline & GRE & $0.3-1.9$ & $1.0 \pm 0.4$ & 1.8 & T1-FFE & $0.3-0.7$ & $0.4 \pm 0.3$ & 1.0 & $* * *$ \\
\hline
\end{tabular}

\section{Local GD}

In CT images, $M A D_{\text {local }}$ ranged from 0.1-0.3 mm with mean $M A D_{\text {local }}$ of $0.2 \pm 0.04 \mathrm{~mm}$ ( Fig.4). All MR images acquired at $7 T$ presented a steady increase in $M A D_{\text {local }}$ with increasing distance from the magnetic isocentre. $M A D_{\text {local }}$ ranged from $0.2-1.8 \mathrm{~mm}$ for 7T-MRI and from 0.1-0.7 $\mathrm{mm}$ for 3T-MRI. Each 7T-MR sequence remained below the clinical tolerance-level of $1 \mathrm{~mm}$ up until a defined DSV (Table 2). For T1-GRE, $M A D_{\text {local }}$ exceeded $1 \mathrm{~mm}$ only at DSV of $87.5-88.7 \mathrm{~mm}$. 
For all $3 T M R$ sequences, $M A D_{\text {local }}$ remained below $1 \mathrm{~mm}$ throughout the whole VOI. MAD $D_{\text {local }}$ of MP2RAGE was statistically greater than all other 7T MR sequences $\left(P<0.001\right.$, Fig. S.2). No significant difference in $M D_{\text {local }}$ was found between T2-SPACE, T2- SPACE FLAIR ( $P=0.064)$, and T1-GRE $(P=0.093)$, respectively. Within the 3T dataset, $M A D_{\text {local }}$ of 3D TFE was significantly different from T2-VISTA $(P<0.001)$, T2-VISTA FLAIR $(P=0.046)$, and T1-FFE $(P<0.001)$. T2-VISTA FLAIR presented significantly different $M A D_{\text {local }}$ from T1-FFE $(P<0.001)$. MAD local was not significantly different between T1-FFE and T2-VISTA $(P=0.51)$, and the T2-VISTA and T2-VISTA FLAIR sequences $(P=0.061)$. However, all sequences met the criteria for $G D$ and seemed to have found an equilibrium with requirements for anatomical imaging.

Statistical analyses of $M A D_{\text {local }}$ between equivalent $7 T$ and 3T-sequences indicated significant differences for all sequences $(p<0.001)$. However, no significant difference in $M A D_{\text {local }}$ was found between equivalent sequences when the DSV was considered (Table 2).

Table 2: Local geometrical deformation as measured on $3 T$ and $7 T M R I ~^{* * *}=P<0.001$, $\S=D S V$ at which statistical significance between sequences occurred)

\begin{tabular}{|c|c|c|c|c|c|c|c|}
\hline \multirow[b]{2}{*}{$\mathrm{B}_{0}$} & \multicolumn{7}{|l|}{ MAD ${ }_{\text {Local }}($ in $\mathrm{mm})$} \\
\hline & Sequence & \multicolumn{2}{|c|}{ Range } & \multicolumn{2}{|c|}{ Mean $\pm s d$} & $\left|\mathrm{Cl}_{95}\right|$ & $<1 \mathrm{~mm}$ at DSV \\
\hline \multirow[t]{5}{*}{ 7T } & MP2RAGE & \multicolumn{2}{|c|}{$0.22-1.81$} & \multicolumn{2}{|c|}{$0.85 \pm 0.30$} & 1.45 & 68.6 \\
\hline & SPACE & \multicolumn{2}{|c|}{$0.21-1.08$} & \multicolumn{2}{|c|}{$0.68 \pm 0.28$} & 1.23 & 101.7 \\
\hline & SPACEFLAIR & \multicolumn{2}{|c|}{$0.27-1.24$} & $0.61 \pm 0.28$ & & 1.17 & 92.5 \\
\hline & GRE & \multicolumn{2}{|c|}{$0.24-1.09$} & $0.68 \pm 0.27$ & & 1.23 & 87.5-88.7 \\
\hline & \multicolumn{5}{|l|}{$\mathrm{MAD}_{\text {Local }}$ (in mm) } & \multicolumn{2}{|r|}{$P$ value } \\
\hline $\mathrm{B}_{0}$ & Sequence & Range & Mean $\pm \mathrm{sd}$ & $\left|\mathrm{Cl}_{95}\right|$ & $<1 \mathrm{~mm}$ at DSV & Sign. & $\mathrm{P}<0.05$ at DSV \\
\hline \multirow[t]{4}{*}{$3 \mathrm{~T}$} & 3DTFE & $0.11-0.63$ & $0.24 \pm 0.19$ & 0.62 & all & $* * *$ & 42.4 \\
\hline & T2-VISTA & $0.23-0.45$ & $0.38 \pm 0.26$ & 0.90 & all & $* * *$ & 68.7 \\
\hline & T2-VISTA FLAIR & $0.14-0.43$ & $0.21 \pm 0.17$ & 0.55 & all & $* * *$ & 47.4 \\
\hline & T1-FFE & $0.21-0.73$ & $0.35 \pm 0.28$ & 0.91 & all & $* * *$ & $45-56.1$ \\
\hline
\end{tabular}

\section{D displacement}

The magnitude of each displacement vector is presented in a 2D mesh-plot in axial $(\mathrm{XZ})$, coronal $(\mathrm{XY})$, and sagittal $(\mathrm{YZ})$ planes, intersecting the magnetic isocentre. The worst GD was presented in MP2RAGE and 3D TFE (Fig.4). Additionally, anisotropic distributions of GD were observed along the phase-encoding direction and frequency-encoding direction. Particularly the anterior-superior section was affected by spatial deformation and maximal GD was observed in the right-superior section of the anterior region (frontal lobe). Distortion maps of all sequences are presented in Figures S.3-5. 


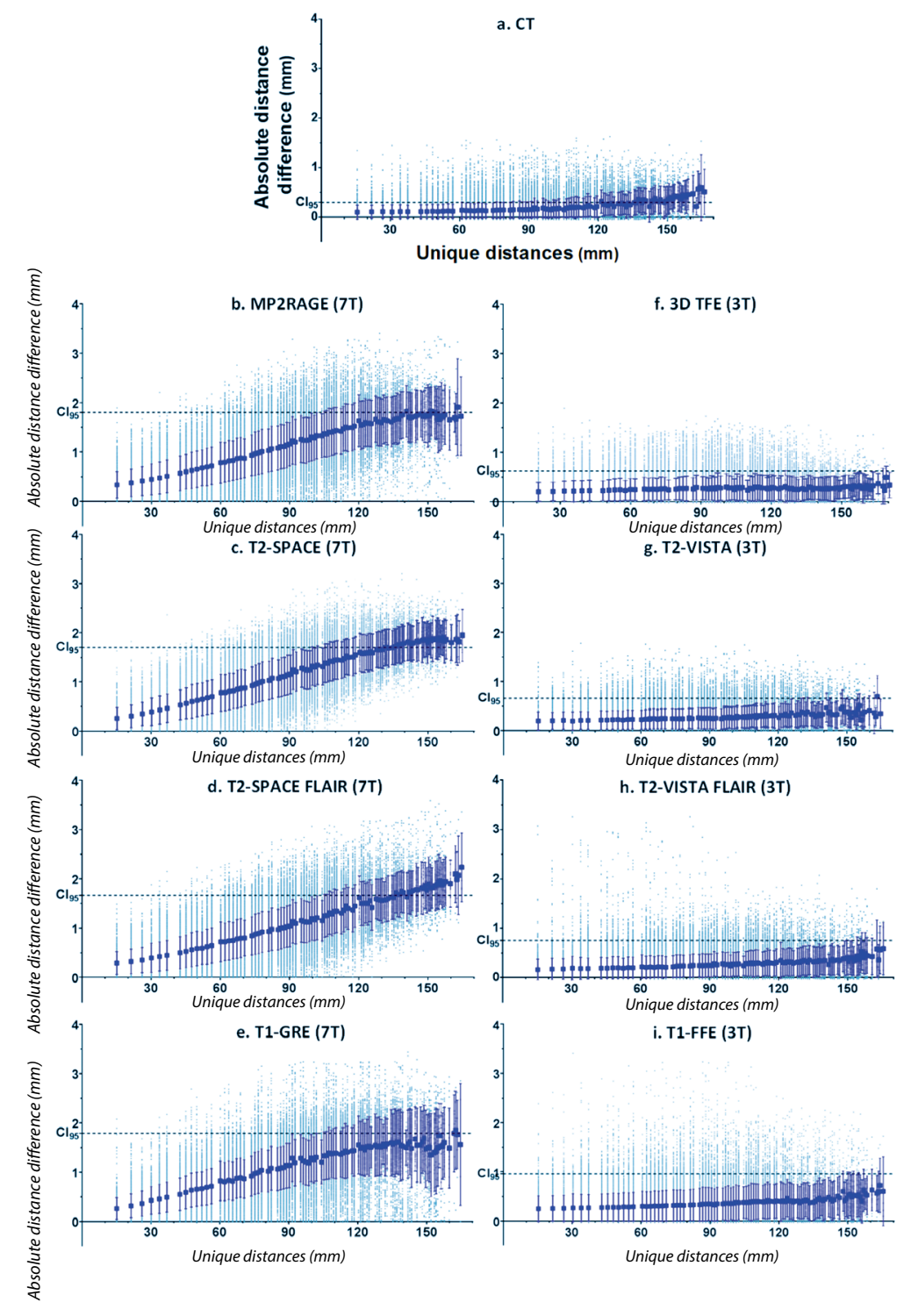

Fig. 2: Absolute differences in Euclidian distance between the measured and reference dataset (grey dot) relative to the unique distances found in the reference dataset, observed within CT (a), MP2RAGE (b), T2-SPACE (c), T2-SPACE FLAIR (d), T1-GRE (e), 3D TFE (f), T2-VISTA (g), T2-VISTA FLAIR (h), T1-FFE (i). The overall geometric distortion was quantified by $M A D_{\text {global }}( \pm S D)$ (blue square). The $95 \%$ confidence interval $\left(\mathrm{Cl}_{95}\right)$ is shown as the dotted horizontal line 

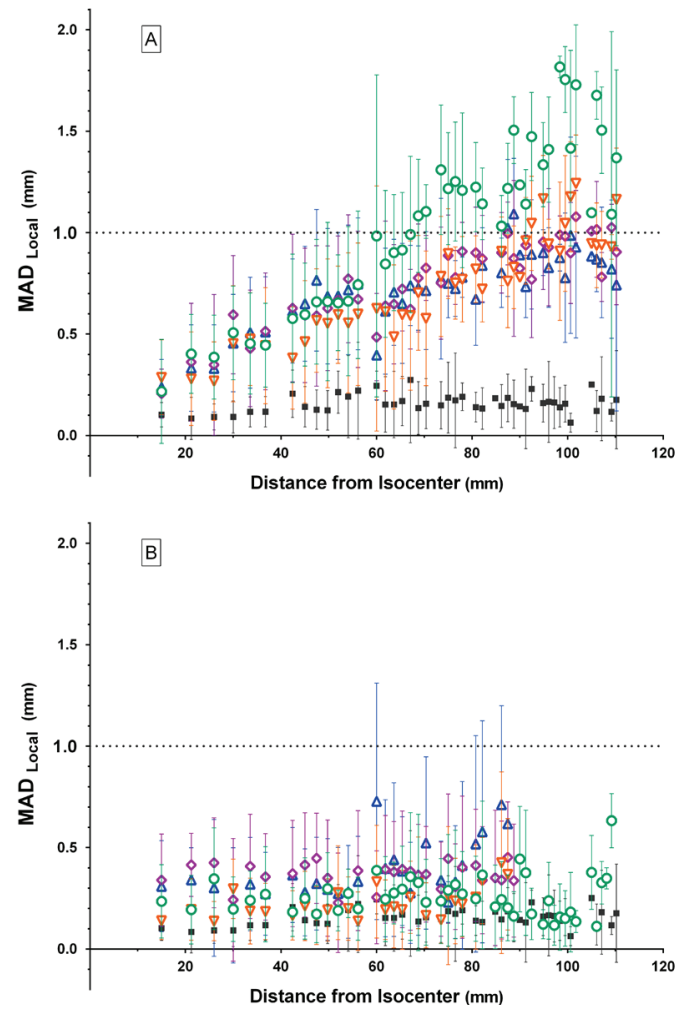

Fig. 3: $M A D_{\text {Local }}$ values $( \pm S D)$ relative to the distance from the magnetic isocentre at $7 T$ (a) and $3 T$ (b) MRI, both relative to $C T$ (black square). Presented $7 T M R$ sequence include MP2RAGE (green circle), T2-SPACE (purple diamond), T2-SPACE FLAIR (orange downward triangle), and T1-GRE (blue upward triangle). The same colour- and shape-code was used for the equivalent 3T sequences, 3D TFE, T2-VISTA, T2-VISTA FLAIR, and T1-FFE, respectively. The dotted horizontal line represents the $1 \mathrm{~mm}$-acceptability level required for spatially reliable RTP 

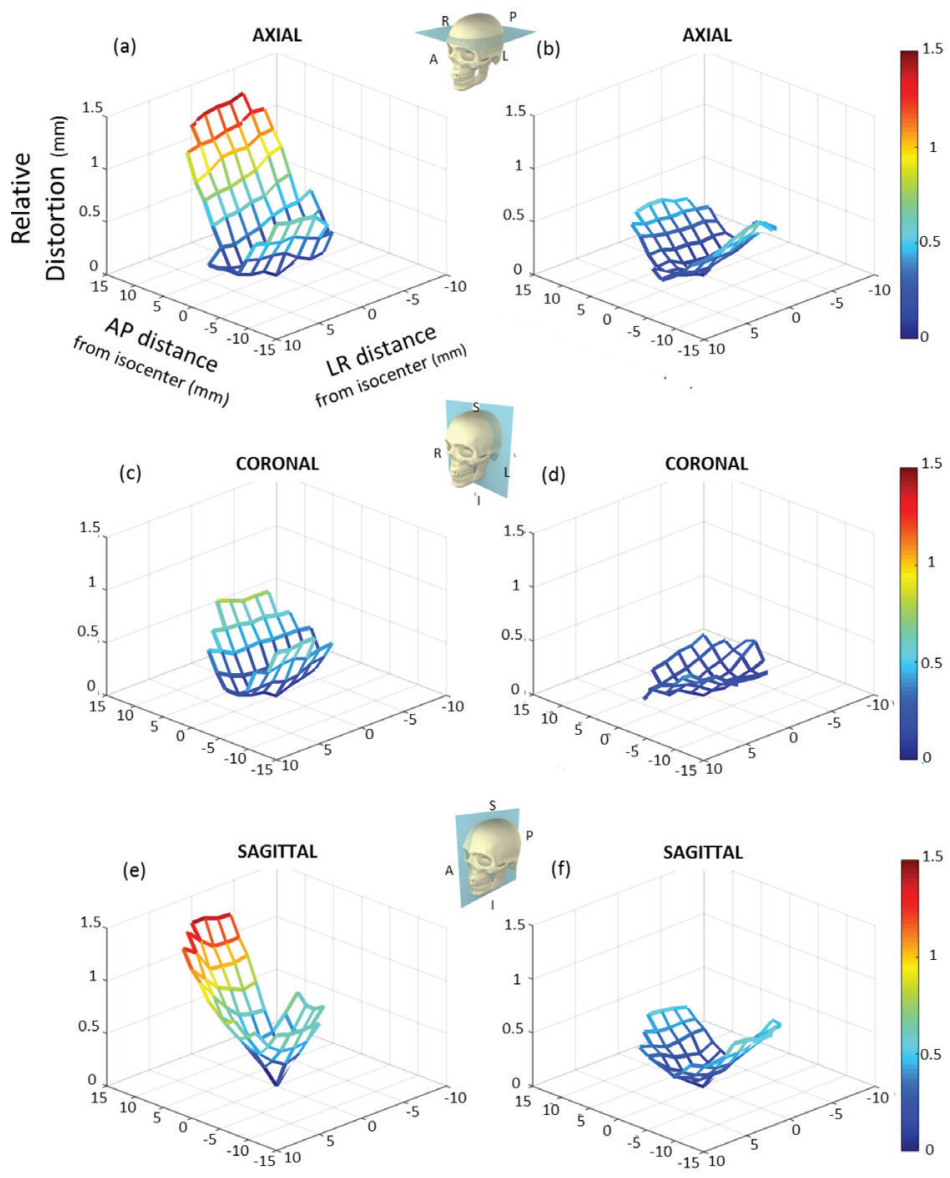

Fig. 4: Distortion maps of MP2RAGE on 7TMRI (a) and 3D TFE on 3TMRI (b) measured in the axial plane nearest the magnetic isocentre $(Y=0)$, in the coronal plane $(Z=0)$, and in the sagittal plane $(X=0)$ 


\section{Discussion}

This study reports both system- and phantom-related GD on clinically-relevant MRsequences at 7T-MRI for neurological imaging and allows direct comparison to GD on both 3T-MRI and CT-images. System-related GD was present in all 3T- and 7T-MRI and increased with increasing distance from the magnetic isocentre, even though vendor-provided correction methods and $2^{\text {nd }}$ order 3D shimming were applied. However, GD of $\leq 1 \mathrm{~mm}$ could be assured within a sequence-dependent DSV near the magnetic isocentre.

While UHF-MRI promises clinical gain in terms of spatial resolution, SNR, contrast, and delineation of pathology, implementation of 7T-MRI in RTP requires $\mathrm{mm}$ scale spatial accuracy for reliable, high-precision radiotherapy. This geometrical requirement was met by all 3T-MR sequences throughout the entire VOI while the magnitude of GD in 7T-MRI depended on the distance from the magnetic isocentre and applied sequence. GD $\leq 1 \mathrm{~mm}$ was apparent in 7T-MRI within a sequence-specific DSV. Moreover, no significant differences in system-related GD were found between 3T- and 7T-images near the magnetic isocentre. This implies that these 7T-MRI with clinically-acceptable/tolerable levels of spatial uncertainty could be applied for highprecision RTP in central brain regions, under the assumption that tissue-related GD is negligible. In addition, it has previously been shown that GD in 7T-MRI did not affect targeting of basal ganglia and subthalamic nuclei in deep-brain stimulation ${ }^{23,31}$.

Some sequences are more prone to GD than others as gradient pulse scheme and parameter settings affect $\mathrm{B}_{0}$-homogeneity and eddy currents ${ }^{25,29,32}$. Our MP2RAGE sequence, which acquires 2 gradient-echo images at different inversion times, presented the largest GD at 7T (and smallest DSV where GD $<1 \mathrm{~mm})^{33}$. Nevertheless, even in this worst-case scenario, system-related GD at 7T was below the clinically acceptable limit of $\leq 1 \mathrm{~mm}$ within $68.7 \mathrm{~mm}$ DSV. Presumably, GD in MP2RAGE could be related to the combination of different sequence-parameter settings (receiver bandwidth (rBW), FOV, and matrix size $)^{13,14,25,29,31,32,34}$. The effect of varying rBW on GD and SNR has extensively been studied by Walker et al. (2014) in 4 different MR-systems ${ }^{31}$. For instance, increasing rBW would reduce GD, chemical shift, and susceptibility artefacts but would also reduce SNR. Limiting FOV-settings (i.e., fewer acquisition lines in k-space) could subside the impact of time-dependent off-resonance effects, reducing $\mathrm{GD}^{34,35}$. However, this could also influence spatial resolution, SNR, and induce potential fold-over artefacts.

Residual GD in phase-encoding direction is expected to be related to gradient nonuniform and could be adequately reduced by vendor-installed distortion correction methods ${ }^{14,18,25,26}$. The technique behind this correction is similar for all main MRmanufacturers and achieve comparable and reproducible results for 3T- and 7T-MR| $\left.\right|^{4,14,15,18,31}$. Nonetheless, some residual system-dependent GD was detected in 
this study. It is therefore important to note that vendor-provided distortion correction is not flawless in correcting for B0-inhomogeneity and eddy currents, and gradient coils are designed with a tolerated performance variation ${ }^{24,36}$. For example, general gradient errors of body gradient coils should be $>2 \%$ of the gradient strength over a $40 \mathrm{~cm} \mathrm{DSV}^{37,38}$.

Our findings of GD corresponded well with literature. At 3T, Stanescu et al. (2010) reported maximal overall GD of $0.6 \mathrm{~mm}$ in 3DTFE sequences and Schmidt et al. (2016) have shown global GD of less than $0.5 \mathrm{~mm}$ in an IR-sequence $(r B W=890 \mathrm{~Hz} /$ pixel mm, resolution $\left.1 \mathrm{~mm}^{3}\right)^{14,39}$. However, some studies report GD of several centimetres ${ }^{25-27}$. These findings, however, occurred at the edge of the scanner's FOV on a large cubical phantom that was placed in a body-coil. In contrast to this, we used a dedicated anthropomorphic head phantom to mimic a clinically realistic scan-procedure with standard RF-coils, shimming, distortion correction, and FOV settings ( $\leq 256 \mathrm{~mm}$ ).

Similar to our approach and FOV setting, Dammann et al. (2011) investigated hardware-related GD in 7T-MRI using a cylindrical phantom (190 POIs) and presented local GD smaller than $1 \mathrm{~mm}$ at $<80 \mathrm{~mm}$ DSV, except in T2-VISTA ( $\mathrm{rBW}=350 \mathrm{~Hz} /$ pixel $625 \mathrm{~Hz} / \mathrm{mm}$, resolution $0.56 \mathrm{~mm}^{2}$ ) that showed maximal local GD of $1.6 \mathrm{~mm}^{26}$. Presumably, this sequence was affected by B1-inhomogeneities and measurements at the phantom's edge which could explain our finding of $1.2 \mathrm{~mm}$ local GD at 80 $\mathrm{mm}$ DSV. Differences in rBW-setting could explain GD differences found in MPRAGE $\left(\mathrm{rBW}=600 \mathrm{~Hz} / \mathrm{mm}\right.$, resolution $\left.1 \mathrm{~mm}^{3}\right)$ and MP2RAGE $\left(\mathrm{rBW}=354 \mathrm{~Hz} / \mathrm{mm}, 0.7 \mathrm{~mm}^{3}\right)$. Recently, Lau et al. (2017) demonstrated differences in local GD between 3T and 7T larger than $1 \mathrm{~mm}$ at $80 \mathrm{~mm}$ DSV for T1-MPRAGE $\left(\mathrm{rBW}=244 \mathrm{~Hz} / \mathrm{mm}, 0.8 \mathrm{~mm}^{3}\right)^{4}$. In our study, MP2RAGE showed differences in $M A D_{\text {local }}$ between $3 T$ and $7 T$ to be below 1 $\mathrm{mm}$ up for a $\leq 87.5 \mathrm{~mm}$ DSV.

Distortion maps of MP2RAGE indicated an anisotropic distribution of GD along the phase-encoding (AP) and frequency-encoding (SI) direction (Fig.4). In a comparable 7T phantom study by Cho et al. (2010), coronal distortion maps showed $0.4 \mathrm{~mm} \mathrm{GD}$ along the frequency-encoding direction and $0.8 \mathrm{~mm}$ along the phase-encoding direction ${ }^{22}$.

We observed GD in CT-images with $M A D_{\text {global }}$ ranging from $0.1-0.6 \mathrm{~mm}$ and $M A D_{\text {local }}$ ranging from $0.1-0.3 \mathrm{~mm}$. These findings were unanticipated as it is generally recognized that CT-images are distortion-free. Other studies have merged MRI with $\mathrm{CT}$, ignoring this innate $\mathrm{GD}$ and measured fundamental errors. Artefacts in CT-images could originate from incorrectly reported scanner table-speed or shearing distortion (incorrectly reported gantry-tilt or table-bending). In this study, GD in CT-images could represent partial volume effects and variations in manual reconstruction leading to slightly different xyz-coordinates measurements of POls (experimental inaccuracies). 
Inthis study, we limited ourselves to evaluating system-related GD and not tissue-related GD. Relative to system-related GD, tissue-induced GD is rather small in 3T-sequences that are currently being used for RTP of brain tumours, but it is nevertheless not negligible ${ }^{16}$. Simulations on 3T-MRI suggest that susceptibility-induced GD were on average $0.6 \mathrm{~mm}$ at cranial air-cavities 40 . This has recently been confirmed by Schmidt et al. (2016), who specified susceptibility-related GD of $0.4 \mathrm{~mm}$ in the naso-oropharyngeal cavities and around the internal ear canal using a T1-weighted sequence ( $r B W=890$ $\mathrm{Hz} / \mathrm{mm}$, resolution $\left.1 \mathrm{~mm}^{3}\right)^{14}$. It remains to be determined what the impact of tissuerelated GD is for 7T-MRI. Susceptibility artefacts are proportional to the magnetic field strength and TE but could be reduced by increasing rBW12. Regardless, patientspecific correction methods are required ${ }^{16,37,40}$. Recently, Rai et al. (2018) developed an 3D printed human skull with sinuses and mastoid air cells, water-based brain tissue and eyes and surrogate cortical bone41. Such realistic anatomical skulls could be used to further assess tissue-induced GD in simulated air cavities, bony anatomy, and soft tissue. Since system-related GD could be further reduced by appropriate setting of MR-sequence parameters, research has been performed to systematically assess the impact of e.g., rBW, FOV, matrix size, scanning direction, and various (post-)processing correction algorithms ${ }^{16,18,27,31,36}$. However, 7T-MR protocols need to be optimised specifically for each clinical application and the trade-off between anatomical imagequality (spatial resolution, SNR, visibility of pathology) and image integrity (spatial accuracy) needs to be made, while respecting specific absorption rate restriction ${ }^{29,32}$. For example, high-precision RTP relies heavily on high geometrical accuracy but could compensate on image-resolution, while in radiology larger GD could be tolerated.

To apply 7T-images in radiotherapy, GD should be $\leq 1 \mathrm{~mm}$ to ensure reliable RTP and dose delivery with local dose variations less than $5-10 \% 21$. This strict criterion was met by all the clinically relevant sequences we tested on 3T- and 7T-MRI up until 92.5$101.7 \mathrm{~mm}$ DSV around the magnetic isocentre, except for the MP2RAGE sequence. Delineations of brain lesions and organs-at-risk in the frontal or occipital lobe on 7T-MRI can be significantly influenced by GD as the spatial uncertainty exceeds 1 $\mathrm{mm}$ in these regions. Even though systematic and random treatment uncertainties are foreseen in a planning treatment volume (PTV), the degree and location of GD is rarely taken into account during radiation dose planning. This study has shown that system-related GD should be incorporated into the PTV-margin to achieve a high level of dose delivery accuracy for intracranial (stereotactic) radiotherapy. This can be accomplished by incorporating the GD present at the tumour location within a margin recipe for dose delivery as suggested by Van Herk et al. (2004) and Seravelli et al. (2015) ${ }^{42,43}$. We therefore recommend applying larger tumour site-specific PTVmargins in regions where spatial integrity of 7T-MRI could not be warranted within 1 $\mathrm{mm}$. Apart from adjustment of PTV-margins, it is advised to find a trade-off between image quality and image distortion, and apply MR-imaging protocols that are dedicated for RTP purposes. 
In conclusion, GD of $\leq 1 \mathrm{~mm}$ could be assured within a sequence-dependent DSV near the magnetic isocentre, implying that this 7T-MRI protocol could be applied with clinically acceptable/tolerable levels of spatial uncertainty for high-precision RTP in central brain regions, under the assumption that tissue-related GD can be ignored. For peripheral regions, 7T-MR protocol for RTP should incorporate GD in tumoursite specific PTV-margins for treatment uncertainties. Nevertheless, dedicated MRprotocols are aspired for application in radiation oncology and further optimisation of sequence parameter settings and GD reduction methods are needed. 
1. Niyazi M, Brada M, Chalmers AJ, et al. ESTRO-ACROP guideline"target delineation of glioblastomas". Radiother Oncol. 2016;118(1):35-42.

2. Claes $A$, Idema AJ, Wesseling P. Diffuse glioma growth: a guerilla war. Acta neuropathologica. 2007;114(5):443-458.

3. Barrett TF, Sarkiss CA, Dyvorne HA, Lee J, Balchandani P, Shrivastava RK. Application of Ultrahigh Field Magnetic Resonance Imaging in the Treatment of Brain Tumors: A Meta-Analysis. World neurosurgery. 2016;86:450-465.

4. Lau JC, Khan AR, Zeng TY, MacDougall KW, Parrent AG, Peters TM. Quantification of local geometric distortion in structural magnetic resonance images: Application to ultra-high fields. Neuroimage. 2017.

5. Trattnig S, Bogner W, Gruber S, et al. Clinical applications at ultrahigh field (7 T). Where does it make the difference? NMR Biomed. 2016;29(9):1316-1334.

6. Trattnig S, Springer E, Bogner W, et al. Key clinical benefits of neuroimaging at 7T. Neuroimage. 2016.

7. Christoforidis GA, Yang M, Abduljalil A, et al. "Tumoral pseudoblush" identified within gliomas at high-spatial-resolution ultrahigh-field-strength gradient-echo MR imaging corresponds to microvascularity at stereotactic biopsy. Radiology. 2012;264(1):210-217.

8. Moenninghoff $C$, Maderwald $S$, Theysohn JM, et al. Imaging of adult astrocytic brain tumours with 7 T MRI: preliminary results. Eur Radiol. 2010;20(3):704-713.

9. Compter I, Peerlings J, Eekers DB, et al. Technical feasibility of integrating $7 \mathrm{~T}$ anatomical MRI in image-guided radiotherapy of glioblastoma: a preparatory study. MAGMA. 2016;29(3):591-603.

10. Bian W, Hess CP, Chang SM, Nelson SJ, Lupo JM. Susceptibility-weighted MR imaging of radiation therapy-induced cerebral microbleeds in patients with glioma: a comparison between 3T and 7T. Neuroradiology. 2014;56(2):91-96.

11. Lupo JM, Chuang CF, Chang SM, et al. 7-Tesla susceptibility-weighted imaging to assess the effects of radiotherapy on normal-appearing brain in patients with glioma. Int J Radiat Oncol Biol Phys. 2012;82(3):e493-500.

12. Dietrich O, Reiser MF, Schoenberg SO. Artifacts in 3-T MRI: physical background and reduction strategies. Eur J Radiol. 2008;65(1):29-35.

13. Weygand J, Fuller CD, Ibbott GS, et al. Spatial Precision in Magnetic Resonance Imaging-Guided Radiation Therapy:The Role of Geometric Distortion. Int J Radiat Oncol Biol Phys. 2016;95(4):1304-1316.

14. Schmidt MA, Wells EJ, Davison K, Riddell AM, Welsh L, Saran F. Stereotactic Radiosurgery Planning of Vestibular Schwannomas: Is MRI at 3 Tesla Geometrically Accurate? Med Phys. 2016.

15. Torfeh T, Hammoud R, Perkins G, et al. Characterization of 3D geometric distortion of magnetic resonance imaging scanners commissioned for radiation therapy planning. Magnetic resonance imaging. 2016;34(5):645-653. 
16. Wang $\mathrm{H}$, Balter J, Cao Y. Patient-induced susceptibility effect on geometric distortion of clinical brain MRI for radiation treatment planning on a 3T scanner. Phys Med Biol. 2013;58(3):465-477.

17. Klein EE, Hanley J, Bayouth J, et al. Task Group 142 report: quality assurance of medical accelerators. Med Phys. 2009;36(9):4197-4212.

18. Duchin Y, Abosch A, Yacoub E, Sapiro G, Harel N. Feasibility of using ultra-high field (7 T) MRI for clinical surgical targeting. PLoS One. 2012;7(5):e37328.

19. Jursinic PA, Rickert K, Gennarelli TA, Schultz CJ. Effect of image uncertainty on the dosimetry of trigeminal neuralgia irradiation. Int J Radiat Oncol Biol Phys. 2005;62(5):1559-1567.

20. Karaiskos P, Moutsatsos A, Pappas E, et al. A simple and efficient methodology to improve geometric accuracy in gamma knife radiation surgery: implementation in multiple brain metastases. Int J Radiat Oncol Biol Phys. 2014;90(5):1234-1241

21. Seibert TM, White NS, Kim GY, et al. Distortion inherent to magnetic resonance imaging can lead to geometric miss in radiosurgery planning. Practical radiation oncology. 2016;6(6):e319-e328.

22. Cho $\mathrm{ZH}, \mathrm{Min} \mathrm{HK}$, Oh $\mathrm{SH}$, et al. Direct visualization of deep brain stimulation targets in Parkinson disease with the use of 7-tesla magnetic resonance imaging. J Neurosurg. 2010;113(3):639-647.

23. Wang D, Doddrell DM. Geometric Distortion in Structural Magnetic Resonance Imaging. Current Medical Imaging Reviews. 2005;1(1):49-60.

24. O'Callaghan J, Wells J, Richardson $\mathrm{S}$, et al. Is your system calibrated? MRI gradient system calibration for pre-clinical, high-resolution imaging. PLoS One. 2014;9(5):e96568.

25. Baldwin LN, Wachowicz K, Thomas SD, Rivest R, Fallone BG. Characterization, prediction, and correction of geometric distortion in 3 T MR images. Med Phys. 2007;34(2):388-399.

26. Dammann $\mathrm{P}$, Kraff $\mathrm{O}$, Wrede $\mathrm{KH}$, et al. Evaluation of hardware-related geometrical distortion in structural MRI at 7 Tesla for image-guided applications in neurosurgery. Acad Radiol. 2011;18(7):910-916.

27. Doran SJ, Charles-Edwards L, Reinsberg SA, Leach MO. A complete distortion correction for MR images: I. Gradient warp correction. Phys Med Biol. 2005;50(7):1343-1361.

28. Schenck JF. The role of magnetic susceptibility in magnetic resonance imaging: MRI magnetic compatibility of the first and second kinds. Med Phys. 1996;23(6):815-850.

29. Liney GP, Moerland MA. Magnetic resonance imaging acquisition techniques for radiotherapy planning. Seminars in radiation oncology. 2014;24(3):160-168.

30. Teeuwisse WM, Brink WM, Haines KN, Webb AG. Simulations of high permittivity materials for $7 \mathrm{~T}$ neuroimaging and evaluation of a new barium titanate-based dielectric. Magnetic resonance in medicine. 2012;67(4):912-918. 
31. Walker A, Liney G, Metcalfe P, Holloway L. MRI distortion: considerations for MRI based radiotherapy treatment planning. Australasian physical \& engineering sciences in medicine / supported by the Australasian College of Physical Scientists in Medicine and the Australasian Association of Physical Sciences in Medicine. 2014;37(1):103-113.

32. Kraff O, Fischer A, Nagel AM, Monninghoff C, Ladd ME. MRI at 7 Tesla and above: demonstrated and potential capabilities. J Magn Reson Imaging. 2015; 41(1):13-33.

33. Marques JP, Gruetter R. New Developments and Applications of the MP2RAGE Sequence - Focusing the Contrast and High Spatial Resolution R-1 Mapping. Plos One. 2013;8 (7):11.

34. Hong C, Lee DH, Han BS. Characteristics of geometric distortion correction with increasing field-of-view in open-configuration MRI. Magnetic resonance imaging. 2014;32(6):786-790.

35. Hashemi RH, Bradley Jr WG, Lisanti CJ. MRI: the Basics. 3rd ed. Philadelphia: Lippincott Williams and Wilkins; 2010.

36. Wang D, Strugnell W, Cowin G, Doddrell DM, Slaughter R. Geometric distortion in clinical MRI systems Part II: correction using a 3D phantom. Magnetic resonance imaging. 2004;22(9):1223-1232.

37. Schmidt MA, Payne GS. Radiotherapy planning using MRI. Phys Med Biol. 2015;60(22):R323-361.

38. Jezzard $P$. The physical basis of spatial distortion in magnetic resonance images. In: Bankman IN, ed. Handbook of medical image processing and analysis. 2nd ed. Amsterdam: Elsevier/Academic Press; 2009:499-514.

39. Stanescu T, Jans HS, Wachowicz K, Fallone BG. Investigation of a 3D system distortion correction method for MR images. J Appl Clin Med Phys. 2010;11(1): 200-216.

40. Stanescu T, Wachowicz K, Jaffray DA. Characterization of tissue magnetic susceptibility-induced distortions for MRIgRT. Med Phys. 2012;39(12):7185-7193.

41. Rai $R$, Manton $D$, Jameson MG, et al. 3D printed phantoms mimicking cortical bone for the assessment of ultrashort echo time magnetic resonance imaging. Med Phys. 2018;45(2):758-766.

42. van Herk M. Errors and margins in radiotherapy. Seminars in radiation oncology. 2004;14(1):52-64.

43. Seravalli E, van Haaren PM, van der Toorn PP, Hurkmans CW. A comprehensive evaluation of treatment accuracy, including end-to-end tests and clinical data, applied to intracranial stereotactic radiotherapy. Radiother Oncol. 2015;116(1):131-138. 


\section{Supplementary figures and tables}

Table S.1: Scan parameters of the 7T- and 3T-MR sequences investigated in this study. All sequences were acquired in $3 D$

\begin{tabular}{ccccc} 
7T MRI sequences & MP2RAGE & T2-SPACE & T2-SPACE FLAIR & T1-GRE \\
\hline Imaging plane & Sag & Sag & Sag & Tra \\
Read-out direction & AP & AP & AP & RL \\
Repetition time T (ms) & 5000 & 4000 & 8000 & 33 \\
Echo time TE (ms) & 2.5 & 283 & 302 & 2.5 \\
Inversion time (ms) & TI1 $900-$ TI2 & N/A & 2330 & N/A \\
Turbo factor & 2750 & & & N/A \\
Field of View (mm) & $223 \times 223 \times 240$ & $192 \times 192 \times 288$ & $193 \times 206 \times 208$ & $160 \times 223 \times 208$ \\
Acquisition matrix (voxel) & $320 \times 320 \times 340$ & $320 \times 320 \times 480$ & $240 \times 256 \times 260$ & $320 \times 320 \times 300$ \\
In-plane resolution & $0.7 \times 0.7 \times 0.7$ & $0.6 \times 0.6 \times 0.6$ & $0.8 \times 0.8 \times 0.8$ & $0.5 \times 0.7 \times 0.7$ \\
(mm/voxel) & & & & 11 \\
Nominal flip angle ( ${ }^{\circ}$ ) & $5 \& 3$ & variable & variable & 580 \\
$\begin{array}{c}\text { Bandwidth (Hz/mm) } \\
\text { Acceleration }\end{array}$ & 354 & 620 & 479 & 2 \\
factor (iPAT) & 3 & 4 & 4 & $08: 33$ \\
$\begin{array}{c}\text { Acquisition time } \\
\text { (minutes) }\end{array}$ & $08: 02$ & $07: 50$ & $10: 58$ & \\
\hline
\end{tabular}

\begin{tabular}{ccccc} 
3T MRI sequences & 3D TFE & T2-VISTA & T2-VISTA FLAIR & T1-FFE \\
Imaging plane & Tra & Sag & Sag & Tra \\
Read-out direction & AP & AP & AP & RL \\
Repetition time TR (ms) & 8.1 & 2500 & 8000 & 11 \\
Echo time TE (ms) & 3.7 & 243 & 331.5 & 4.6 \\
Inversion time (ms) & 800 & N/A & 2400 & N/A \\
Turbo factor & 188 & 133 & 110 & N/A \\
Field of View (mm) & $256 \times 256 \times 180$ & $256 \times 256 \times 180$ & $256 \times 256 \times 180$ & $256 \times 192 \times 180$ \\
Acquisition matrix (voxel) & $256 \times 256 \times 180$ & $256 \times 256 \times 180$ & $228 \times 226 \times 150$ & $256 \times 256 \times 180$ \\
In-plane resolution & $1 \times 1 \times 1$ & $1 \times 1 \times 1$ & $1.1 \times 1.1 \times 1.2$ & $1 \times 0.7 \times 1$ \\
(mm/voxel) & & & & \\
Nominal flip angle $\left(^{\circ}\right)$ & 8 & 90 & variable & 8 \\
Bandwidth (Hz/mm) & 191 & 1005 & 346 & 411 \\
$\begin{array}{c}\text { Acceleration factor } \\
\text { (SENSE) }\end{array}$ & 1 & 2 & 2 & 1 \\
Acquisition time & $06: 00$ & $05: 42$ & $08: 24$ & $06: 21$ \\
(minutes) & & & & \\
\hline
\end{tabular}




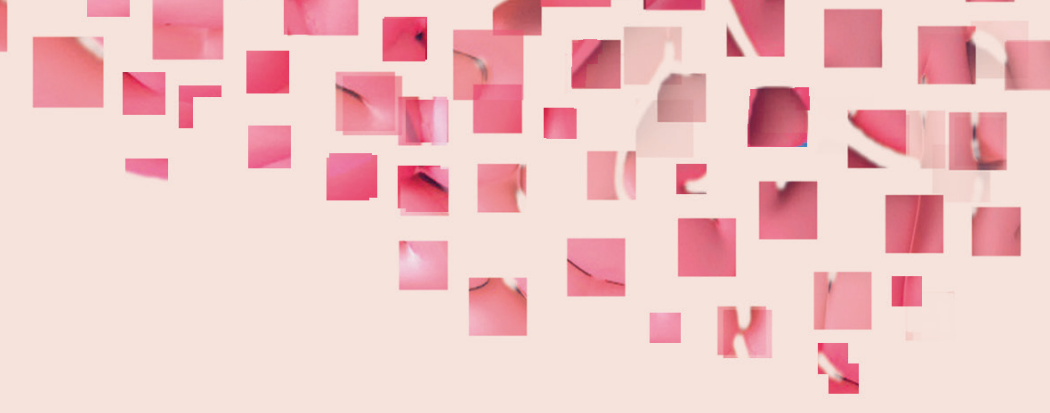

Part III

Autophagy inhibition 


\section{Chapter 6 - Chloroquine combined with concurrent radiotherapy and temozolomide for newly diagnosed glioblastoma: A phase IB trial}

Inge Compter, Daniëlle B.P. Eekers, Ann Hoeben, Kasper M.A. Rouschop, Bart Reymen, Linda Ackermans, Jan Beckervordersantforth, Noël J.C. Bauer, Monique M. Anten, Pieter Wesseling, Alida A. Postma, Dirk De Ruysscher, Philippe Lambin Autophagy. 2020 Sep 20:1-9. 


\section{Abstract}

Treatment of glioblastoma xenografts with chloroquine results in macroautophagy/ autophagy inhibition, resulting in a reduction of tumor hypoxia and sensitization to radiation. Preclinical data show that EGFRvIll-expressing glioblastoma may benefit most from chloroquine because of autophagy dependency. This study is the first to explore the safety, pharmacokinetics and maximum tolerated dose of chloroquine in combination with radiotherapy and concurrent daily temozolomide in patients with a newly diagnosed glioblastoma. This study is a single-center, open-label, dose-finding phase I trial. Patients received oral chloroquine daily starting one week before the course of chemoradiation (temozolomide $75 \mathrm{mg} / \mathrm{m}^{2} / \mathrm{d}$ ) until the end of radiotherapy ( $59.4 \mathrm{~Gy} / 33$ fractions). Thirteen patients were included in the study ( $\mathrm{n}$ $=6: 200 \mathrm{mg}, \mathrm{n}=3: 300 \mathrm{mg}, \mathrm{n}=4: 400 \mathrm{mg}$ chloroquine). A total of 44 adverse events, possibly related to chloroquine, were registered including electrocardiogram QTc prolongation, irreversible blurred vision and nausea/vomiting resulting in cessation of temozolomide or delay of adjuvant cycles. The maximum tolerated dose was $200 \mathrm{mg}$ chloroquine. Median overall survival was 16 months (range 2 - 32). Median survival was 11.5 months for EGFRvIII- patients and 20 months for EGFRvIII+ patients. A daily dose of $200 \mathrm{mg}$ chloroquine was determined to be the maximum tolerated dose when combined with radiotherapy and concurrent temozolomide for newly diagnosed glioblastoma. Favorable toxicity and promising overall survival support further clinical studies. 


\section{Introduction}

Glioblastoma (GBM) is the most common malignant primary brain tumor with an estimated incidence of 4.27 per 100.000 in Europe'. Despite extensive surgery, radiotherapy and temozolomide (TMZ), the prognosis remains poor with a median overall survival (OS) of approximately 15 months and a five-year OS of $5-10 \%{ }^{2}$. None of the attempts to improve the prognosis, including radiation dose escalation, targeted agents and immunotherapy, have changed the dismal outlook ${ }^{3}$. GBM are known to be one of the most heterogeneous tumors in humans with both major inter- and intratumoral variation ${ }^{4}$. Molecular biomarkers such as MGMT (O-6methylguanine-DNA methyltransferase) promotor methylation, 1p/19q co-deletion and IDH1/2 (isocitrate dehydrogenase (NADP(+)) 1/2) mutation significantly predict patient survival ${ }^{5}$. EGFRvIll (epidermal growth factor receptor mutation type III) expression, a genotype observed in $50-60 \%$ in tumors with amplification of the EGFR gene, is associated with a poor prognosis $s^{6-8}$. This is in part through enhanced repair of DNA double-strand breaks and an elevated hypoxic fraction ${ }^{9,10}$. Tissue hypoxia has been shown to correlate with enhanced tumor cell invasion and poor outcome, independent of treatment modality ${ }^{11}$.

Macroautophagy (hereafter described as "autophagy") is a lysosomal degradation pathway, which allows the cell to recycle amino acids and other nutrients to maintain energy levels, protein synthesis and essential metabolic processes during hypoxic conditions and metabolic stress ${ }^{12}$. During this process, a double-membrane structure is formed in which cellular content is engulfed (autophagosome). The autophagosome fuses with a lysosome to expose its content to the lysosomal degradative enzymes. Resistance to autophagy is one of the major reasons why GBM stem cells are known to be highly resistant to both radiotherapy and chemotherapy ${ }^{13-16}$. In EGFRvIIImutated tumors, autophagy is essential to maintain a viable hypoxic fraction and, when targeted, results in elevated response of the tumor to irradiation ${ }^{10}$.

The lysosomotropic drug chloroquine (CQ) is a 4-aminoquinoline commonly used for the prophylaxis and treatment of malaria, rheumatoid arthritis, liver amoebiasis, sarcoidosis and lupus erythematodes. CQ accumulates in the lysosome and thereby raises the intralysosomal $\mathrm{pH}$, preventing the autophagosome-lysosome fusion essential for autophagy ${ }^{17,18}$. There is mounting pre-clinical evidence that hypoxic cells depend on autophagy for survival and that inhibiting autophagy with CQ can enhance both radiotherapy and chemotherapy cytotoxicity in $\mathrm{GBM}^{19-22}$. Moreover, there is evidence that $C Q$ reduces tumor hypoxia by improving the structural and functional features of the intratumoral blood vessels through vessel normalization via NOTCH1 signaling ${ }^{23}$. CQ can penetrate into the central nervous system and the potential added value of $\mathrm{CQ}$ has been demonstrated in a small randomized controlled trial in GBM treated with radiotherapy and carmustine, which showed a trend towards increased $\mathrm{OS}^{24}$. Based on these pre-clinical and early clinical results CQ has received 
an orphan drug designation for the treatment of glioma in the EU in November 2014 (EU/3/14/1377) and the US in May 2015 (Request number: 15-4750). We propose that combining CQ with radiotherapy and TMZ might increase OS significantly. Taking into account that the intracellular effects of $C Q$ are potentially dose-dependent and the effects of $C Q$ in combination with TMZ have not yet been investigated, the recommended phase two dose of $C Q$ in combination with radiotherapy and TMZ needs to be established. This phase I study explores the safety, pharmacokinetics and maximum tolerated dose (MTD) of CQ in combination with radiotherapy and daily TMZ in patients with a newly diagnosed GBM.

\section{Materials and Methods}

\section{Eligibility}

The main inclusion criteria were histologically confirmed newly diagnosed GBM, age $18-70 \mathrm{y} / \mathrm{o}$ and a World Health Organization (WHO) performance status $\geq 2$. The blood samples of these patients required a neutrophil count of $\geq 1.5 \times 109 / \mathrm{L}$, platelets $\geq 100$ $x 109 / L$, a serum creatinine $\leq 1.5 \times$ upper limit of normal, total bilirubin $\leq 1.5 \times$ upper limit of normal and GPT/alanine transaminase or GOT1/aspartate aminotransferase and AP/alkaline phosphatase $\leq 3 \times$ upper limit of normal. The main exclusion criteria included prior radio- or chemotherapy, recent severe cardiac disease, a history of cardiac arrhythmia, cardiac conduction disturbances or medication potentially causing them, retinal or visual field changes unrelated to the tumor location and the use of concurrent CYP/cytochrome P450 enzyme-inducing drugs.

\section{Study design}

This study was a single-center, open-label, dose-finding phase I trial. Eligible patients received radiotherapy and TMZ (Sun Pharmaceutical Industries Ltd, 15913384) according to the standard clinical protocol. This consists of 33 daily fractions of 1.8 Gy to the tumor which included the region of enhancement plus the resection cavity (if resected) with a $2 \mathrm{~cm}$ margin on magnetic resonance imaging in combination with TMZ $75 \mathrm{mg} / \mathrm{m} 2$ per os quaque die (po qd) and six adjuvant cycles of TMZ 150 - $200 \mathrm{mg} / \mathrm{m} 2$ po qd. CQ (Basic Pharma Manufacturing B.V, IMP10153) was taken daily starting one week before the start of chemo-radiation and ending on the last day of radiotherapy. CQ was initially planned to be escalated in 3 dose-levels (200 $\mathrm{mg}, 400 \mathrm{mg}$ and $600 \mathrm{mg}$ po $\mathrm{qd}$ ), each dose level containing a minimum of 3 and a maximum of 6 patients $(3+3 \text { design })^{52}$. Adverse events (AEs) were assessed using the National Cancer Institute Common Toxicity Criteria (CTC) version 4.03. Dose-limiting toxicities (DLTs) were defined as an absolute neutrophil count $\leq 500 / \mu \mathrm{L}$ for $7 \mathrm{~d}$; febrile neutropenia (neutrophil count less than 500 cells per microliter and fever of $\geq 38.5^{\circ} \mathrm{C}$ ); any grade 4 thrombocytopenia $(<25 \times 109 / L)$, and/or requiring platelet transfusion or bleeding requiring medical intervention; any $\geq$ grade 3 non-hematological toxicity except fatigue, nausea, fever or skin reactions; interruption of radiotherapy or TMZ 
due to toxicity during concurrent chemo-radiation or delay of adjuvant cycles of TMZ. Toxicity was considered a DLT if it was at least possibly related to CQ. If DLT was observed in one out of three initial patients, an additional 3 patients were enrolled at that dose level. In case more than one out of the initial three had DLT, no additional patients were required. The MTD for CQ was defined as one dose level below that at which two or more patients experienced a DLT, or if no DLTs were observed, the highest evaluated dose. Once the recommended phase two dose was identified, 3 additional patients were enrolled in order to confirm treatment tolerability. Safety was assessed by an independent data safety monitoring committee four weeks after treatment of every third subject.

\section{Study assessments}

Baseline assessment included a history and physical examination, WHO performance status, laboratory analysis, electrocardiogram, ophthalmological examination and a tone audiogram. Laboratory analyses were performed weekly during concurrent treatment and monthly thereafter. An electrocardiogram was repeated in the second and final week of study treatment for the first 7 patients. The protocol was amended to weekly ECG surveillance during CQ-treatment for the remainder of patients due to observed toxicity. Ophthalmological examination and a tone audiogram were repeated 4 weeks after the end-of-study treatment and if clinically indicated. Assessment of adverse events and physical and neurological examinations were performed monthly during the adjuvant cycles of TMZ. The blood concentration of $C Q$ was monitored on four time points throughout the regimen and before the adjuvant cycles of TMZ (first and second week and end of chemoradiation and 4 weeks after chemoradiation). CQ was analyzed in serum samples using validated liquid chromatography coupled with tandem mass spectrometry (LC-MS/MS) method. The chromatographic separation was achieved on a reversed-phase Thermo

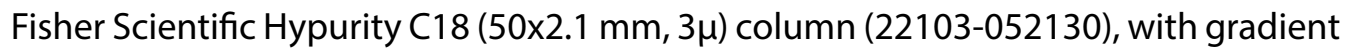
elution using ammonium formate $0.02 \mathrm{~mol} / \mathrm{L} \mathrm{pH} 3.5$ and methanol as mobile phase at a flow rate of $0.5 \mathrm{~mL} / \mathrm{min}$. Protein precipitation with $10 \%$ trichloroacetic acid and D4-CQ was utilized for extraction of CQ from the matrix. CQ was quantitated using MS/MS detection with an electrospray ionization source in positive multiple reaction monitoring (MRM) mode. The MS/MS response was linear over a concentration range from 10 to $2000 \mu \mathrm{g} / \mathrm{L}$ with a correlation coefficient (R2) of 0.999 or better. The withinand between-day precision (\% relative standard deviation) and accuracy were within the acceptable limits as per EMA and FDA guidelines ${ }^{53,54}$. Profile parameters included blood trough level, Area Under the Curve and elimination half-life. Magnetic resonance imaging was performed within $72 \mathrm{~h}$ after brain tumor surgery and at least within 6 weeks prior to registration. Follow-up imaging was performed according to standard clinical protocol on a three-monthly basis after treatment. 


\section{Molecular profiling}

IDH1 or IDH2 mutations were detected with polymerase chain reaction and pyrosequencing, MGMT promoter methylation status was analyzed by bisulfite treatment and methylation-specific polymerase chain reaction followed by pyrosequencing. FISH analysis of $1 \mathrm{p} / 19 \mathrm{q}$ status was performed using the LSI 1p36/1q25 and 19q13/19p13 FISH Probe kit (Abbott Molecular Inc., 04N60-020). To determine EGFRvIll expression, formalin-fixed paraffin-embedded biopsies were probed with antibodies directed against EGFRvIII (clone L8A4, absolute antibody, Ab00184-1.1.) and visualized using HRP-linked anti-mouse (Cell Signaling Technology, 7076S) antibodies) in combination with DAB-staining. Tumor biopsies were scored either negative, less than $1 \%(+)$, between $1 \%$ and $60 \%(++)$ or more than $60 \%(+++)$ of the cells positive in EGFRvIll-expression.

\section{Statistical analysis}

The primaryobjective of thestudy wasto determinetherecommended phasetwo dose. Secondary objectives included evaluation of safety, tolerability, pharmacokinetics and preliminary changes in treatment efficacy. All evaluable patients were included in the analyses. Analysis was limited to presentation of descriptive statistics (e.g., median and range) and frequencies in cross-tabs. Statistical analysis was performed using the statistical program SPSS for Windows (version 22. 0.0, 2013) and R Statistical Software (version 4.0.1). OS was defined as the time from enrolment until death. The survival analyses were performed using the Kaplan-Meier method.

The study was approved by the Medical Review Ethics Committee at Maastricht University Medical Centre +. All procedures performed were in accordance with the ethical standards of the institutional and/or national research committee and with the 1964 Helsinki declaration and its later amendments or comparable ethical standards. Informed consent was obtained from all individual participants included in the study. This trial is registered on clinicaltrials.gov (NCT02378532). 


\section{Results}

Thirteen patients were enrolled in the study between August 2016 and December 2018. The patient and tumor characteristics are summarized in Table 1.

The adverse events $\geq$ CTCAE grade II are presented in Table 2. Fatigue and nausea were the most common reported side effects. No toxic deaths occurred. One patient died due to a thrombotic event shortly after chemoradiation, which was considered unlikely due to $\mathrm{CQ}$. All patients with reported toxicities related to study treatment recovered except for one patient with blurred vision. In 8 patients, eleven Serious Adverse Events (SAE) were reported (Table 3), of which 5 were considered unlikely or not related to $\mathrm{CQ}$. One patient was admitted to the emergency department after a seizure. During hospitalization, he sustained a minor head injury after a fall, which was possibly caused by an increased sedative effect of midazolam after coadministration with CQ, due to reduced metabolism through CYP/cytochrome P450.

At a daily dose of $400 \mathrm{mg} \mathrm{CQ}, 3$ out of 4 patients experienced a DLT, after which the study medication was discontinued. In the final week of study treatment, two patients showed a significant prolongation of the electrocardiogram QT corrected interval (CTCAE grade III). The QT-corrected interval recovered to normal within 1 week after cessation of $C Q$ in one patient but required four weeks to recover for the other. Neither of the two patients experienced any physical complaints due to the cardiac conduction disturbances. Another patient in the $400 \mathrm{mg} \mathrm{CQ}$ cohort developed blurred vision in the second week of chemo-radiation. Ophthalmic examination showed no abnormalities. As an adverse effect from CQ could not be excluded, CQ was stopped. The vision did not recover after cessation of $C Q$ at the end of follow-up 9 months after inclusion. At a daily dose of $300 \mathrm{mg}$ CQ, 2 patients experienced CTCAE grade II and III nausea and vomiting, which resulted in hospital admission in one patient and discontinuation of TMZ in both and was therefore considered a DLT in both cases. At a daily dose of $200 \mathrm{mg} \mathrm{CQ}, 1$ patient developed nausea, vomiting and liver function abnormalities resulting in hospital admission, which was considered a DLT. A daily dosage of $200 \mathrm{mg}$ CQ was therefore considered the MTD.

Valid pharmacokinetic measurements were available from the $200-300 \mathrm{mg}$ cohorts. Median duration of CQ exposure was $52 \mathrm{~d}$. Median CQ plasma concentrations at the expected steady-state (after 2 weeks of CQ) were $192.3 \mu \mathrm{g} / \mathrm{L}(200 \mathrm{mg}$ CQ) and 391.9 $\mu \mathrm{g} / \mathrm{L}$ (300 mg CQ). At the end of radiotherapy, median CQ plasma concentrations were $199.5 \mu \mathrm{g} / \mathrm{L}$ (200 mg CQ) and $482.4 \mu \mathrm{g} / \mathrm{L}$ (300 mg CQ).

Adherence to the study medication was high, with only 2 patients missing 3 and 6 dosages of CQ, respectively. At data cut-off (February 2020), 1 patient had discontinued treatment including chemoradiation at his own request in the first week of treatment due to complaints of nausea and vomiting, without the use of 
anti-emetics. This patient was replaced by another patient, after which follow-up ended. No other patients were lost to follow-up. One patient misunderstood the instructions regarding TMZ and only took it during weekdays.

At the time of analysis, the median follow-up was 32 months. The median OS was 16 months, 95\% Cl [6.5 - 25.5] (Fig. 1). The median OS time was 21 months, 95\% Cl [12.5 -29.5], for patients with an MGMT methylated promoter and 12.5 months, $95 \%$ $\mathrm{Cl}[8.9$ - 16.1], for patients with an unmethylated promoter. An EGFRvIII mutation was present in 7 out of 13 patients. These patients had a median OS of 20 months, $95 \% \mathrm{Cl}$ [13.6 - 26.4], compared to 11.5 months, 95\% Cl [9.1 - 13.9], in non-EGFRvIll-mutated patients (Fig. 2). 
Table 1: Patient and tumor characteristics

\begin{tabular}{|c|c|}
\hline \multicolumn{2}{|l|}{ Patient characteristics } \\
\hline \multicolumn{2}{|l|}{ Age [years] } \\
\hline Median (range) & $58(38-68)$ \\
\hline \multicolumn{2}{|l|}{ Sex } \\
\hline Male & $10(77 \%)$ \\
\hline Female & $3(23 \%)$ \\
\hline \multicolumn{2}{|l|}{ WHO performance status } \\
\hline 0 & $3(23 \%)$ \\
\hline 1 & $4(31 \%)$ \\
\hline 2 & $6(46 \%)$ \\
\hline \multicolumn{2}{|l|}{ Surgical procedure } \\
\hline Biopsy & $3(23 \%)$ \\
\hline Resection & $10(77 \%)$ \\
\hline \multicolumn{2}{|l|}{ Tumor characteristics } \\
\hline MGMT promotor methylation & $5(38 \%)$ \\
\hline \multicolumn{2}{|l|}{ IDH1/2 mutation } \\
\hline $\mathrm{IDH} 1$ & $1(8 \%)$ \\
\hline $\mathrm{IDH} 2$ & $0(0 \%)$ \\
\hline 1p/19q co-deletion & $0(0 \%)$ \\
\hline \multicolumn{2}{|l|}{ EGFRvIll expression } \\
\hline- & $6(46 \%)$ \\
\hline+ & $2(16 \%)$ \\
\hline++ & $0(0 \%)$ \\
\hline+++ & $5(38 \%)$ \\
\hline
\end{tabular}

Methylation of the MGMT (O-6-methylguanine-DNA methyltransferase) promoter, IDH1/2 (isocitrate dehydrogenase (NADP(+)) 1/2) mutation, loss of heterozygosity of $1 p$ and $19 q$ (1p/19q co-deletion), EGFRvIII (epidermal growth factor receptor, mutation type III). Amount of expression detected: negative $(-)$, less than $1 \%(+)$, between $1 \%$ and $60 \%(++)$ or more than $60 \%(+++)$ of the cells positive; World Health Organization (WHO) Performance status 
Table 2. Adverse events grade II - V

\begin{tabular}{|c|c|c|c|c|c|}
\hline & Grade II & Grade III & Grade IV & Grade V & Related to CQ \\
\hline Alanine aminotransferase increased & 1 & & & & Possibly \\
\hline Alopecia & 1 & & & & Unrelated \\
\hline Amnesia & 4 & & & & Unrelated \\
\hline $\begin{array}{l}\text { Aspartate aminotransferase } \\
\text { increased }\end{array}$ & & 1 & & & Possibly \\
\hline Blurred vision & 1 & 1 & & & Probably \\
\hline Confusion & & 1 & & & Unrelated \\
\hline Dermatitis radiation & 1 & & & & Unrelated \\
\hline Diarrhea & & 1 & & & Possibly \\
\hline Dizziness & 1 & & & & Unrelated \\
\hline Dysphasia & & 1 & & & Unrelated \\
\hline $\begin{array}{l}\text { ECG QT corrected interval } \\
\text { prolonged }\end{array}$ & 1 & 2 & & & Possibly/Probably \\
\hline Facial nerve disorder & 1 & & & & Unrelated \\
\hline Fall (Trauma capitis) & 1 & & & & Possibly \\
\hline Fatigue & 6 & 1 & & & Possibly \\
\hline Hallucinations & & & 1 & & Unrelated \\
\hline Headache & 1 & & & & Unrelated \\
\hline Hypercalcemia & 1 & & & & Unlikely \\
\hline Insomnia & 1 & & & & Possibly \\
\hline Nausea & 4 & 2 & & & Possibly \\
\hline Peripheral motor neuropathy & 1 & 1 & & & Unlikely \\
\hline Peripheral sensory neuropathy & 2 & & & & Unrelated \\
\hline Presyncope & 1 & & & & Unrelated \\
\hline Rash maculo-papular & 1 & & & & Possibly \\
\hline Seizure & 1 & & & & Unrelated \\
\hline Thrombolic event & & & 1 & 1 & Unlikely \\
\hline Vomiting & & 1 & & & Possibly \\
\hline Weight loss & 1 & & & & Probably \\
\hline Wound complication, non infectious & 1 & & & & Unrelated \\
\hline
\end{tabular}

Events are listed in alphabetical order and were graded according to National Cancer Institute Common Terminology Criteria for Adverse Events (version 4.03) grade criteria. All counts represent a patient; multiple occurrences of the same adverse event in one individual are counted only once at the highest grade. All grade 2-5 events are shown 
Table 3. Serious Adverse Events

\begin{tabular}{|c|c|c|c|c|}
\hline $\begin{array}{c}\text { Dose level } \\
\text { [mg] }\end{array}$ & SAE & CTCAE grade & Related to CQ & Action taken \\
\hline \multirow[t]{8}{*}{200} & Confusion & III & Unrelated & No adjuvant temozolomide \\
\hline & Seizure & I & Unrelated & Increase dose anticonvulsants \\
\hline & Fall (Trauma capitis) & $\|$ & Possibly & No action \\
\hline & $\begin{array}{c}\text { Thromboembolic } \\
\text { event }\end{array}$ & IV & Unlikely & Anticoagulants \\
\hline & $\begin{array}{c}\text { Thromboembolic } \\
\text { event }\end{array}$ & V & Unlikely & None \\
\hline & Diarrhea & III & Possibly & i.v. fluids \\
\hline & Nausea & III & Possibly & Stop chloroquine \\
\hline & Vomiting & III & Possibly & Stop chloroquine \\
\hline \multirow{3}{*}{300} & & & & Stop chloroquine \\
\hline & Nausea & III & Possibly & Stop temozolomide \\
\hline & & & & Stop radiotherapy \\
\hline \multirow[t]{3}{*}{400} & Hypercalcemia & ॥ & Unlikely & i.v. fluids / bisphosphonates \\
\hline & Blurred vision & III & Probably & Stop chloroquine \\
\hline & Hallucinations & IV & Unrelated & Haloperidol \\
\hline
\end{tabular}

Serious Adverse Events (SAEs) presented per cohort CQ. The adverse events were graded according to National Cancer Institute Common Terminology Criteria for Adverse Events (CTCAEversion 4.03) 


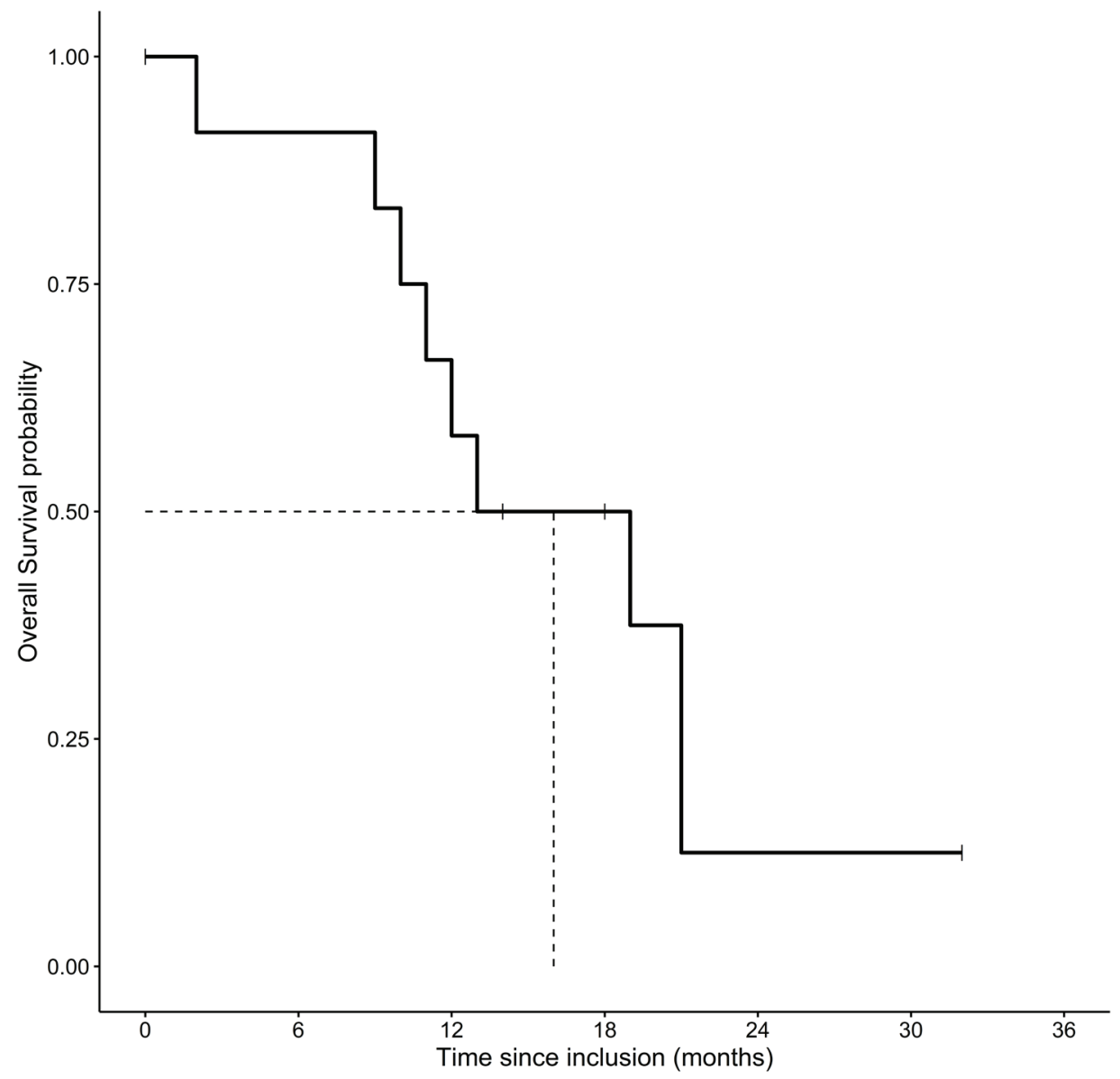

Number at risk (number censored)

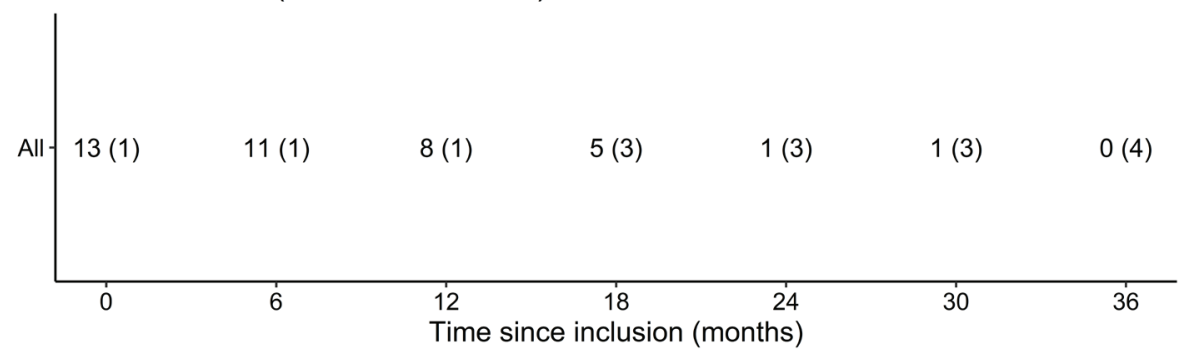

Fig 1. Kaplan-Meier analysis of overall survival of the complete study cohort. Median overall survival is presented by the dotted line 


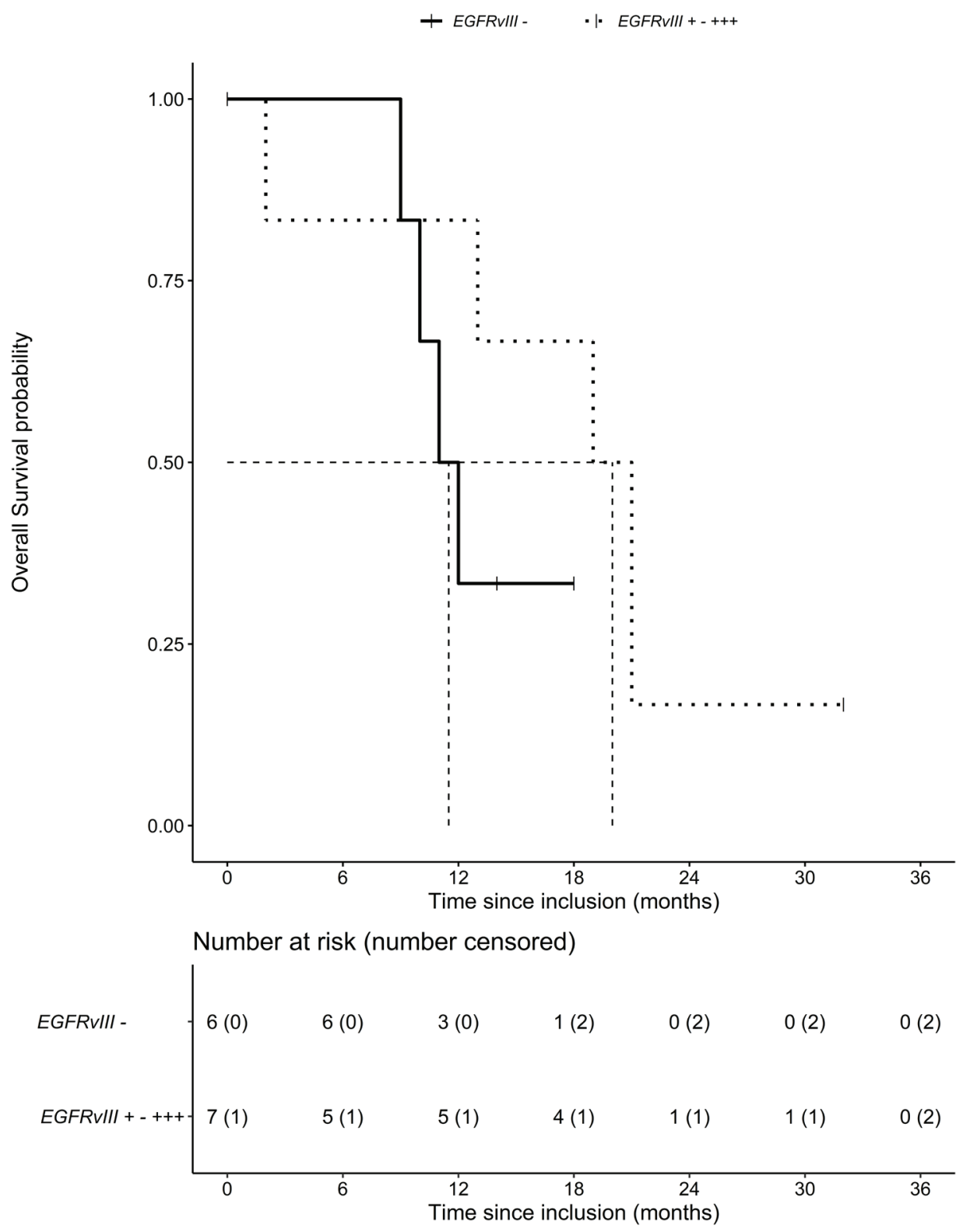

Fig 2. Kaplan-Meier analysis of overall survival split by EGFRvIll amplification status (present or absent). Median overall survival of both groups is presented by the dotted line 


\section{Discussion}

Pre-clinical evidence has shown that patients with a GBM may benefit from adding CQ to chemoradiation, resulting in autophagy inhibition and thereby sensitizing tumor treatment. This phase I dose-escalation trial was conducted to evaluate the safety of CQ when combined with radiotherapy and TMZ. This trial is the first to show that coadministration of $C Q$ in combination with radiotherapy and TMZ is feasible. We have established $200 \mathrm{mg}$ CQ daily as the recommended phase two dose in combination with chemoradiation in GBM.

Although CQ is administered in multiple conditions, and the side effect profile is well established, a significant number of adverse events were observed in this trial. At 400 mg CQ, DLTs included grade III blurred vision and grade III ECG QT-corrected interval prolongation. The complaints of severe blurred vision developed after a cumulative dose of $7.2 \mathrm{~g}$. Retinopathy could not be objectified with ophthalmological examination. Retinal toxicity is a well-known complication due to CQ and its derivatives, which usually occurs at high daily doses, taken to high cumulative doses for a prolonged period of ingestion (years) ${ }^{25}$. Cardiac conduction disturbances, including QT-corrected interval prolongation, are also a recognized adverse effect of CQ-use. Risk factors for cardiotoxicity include renal insufficiency, older age, pre-existing cardiac disease, elevated per-kilogram daily dose and prolonged CQ treatment ${ }^{26}$. Neither of the patients had prior cardiac disease or renal insufficiency and therefore the only risk factor was an elevated CQ-dose. Nausea and vomiting have been well-established side effects of CQ. Moreover, the tumor, postoperative complications and the chemoradiation may have aggravated these complaints.

Several other groups have published clinical trials adding CQ to glioma treatment. Bilger et al treated 5 patients with a recurrent GBM with $250 \mathrm{mg}$ CQ daily and re-irradiation without TMZ, which was well-tolerated and no CQ-related toxicity was observed ${ }^{27}$. Sotelo et al. combined CQ (150 mg po qd) with radiotherapy and carmustine after surgery ${ }^{24}$. In this single-center trial, 30 patients receiving surgery, chemotherapy and radiotherapy were randomized to receive CQ or placebo for 12 months. The median OS was 24 months for the CQ-treated patients and 11 months for controls. No significantCQrelated toxicity was observed. The difference in survival time was statistically significant when compared to control subjects in their later published institutional experience ${ }^{28}$. Rosenfeld et al published a phase I-II trial in which resected GBM patients received the CQ derivative hydroxychloroquine (HCQ) in doses of $200-800 \mathrm{mg}$ po qd with radiation therapy and concurrent TMZ followed by adjuvant $\mathrm{TMZ}^{29}$. Pharmacokinetic analysis showed a significant therapy-associated increase in autophagic vacuoles and LC3B in the peripheral blood mononuclear cells, which was correlated with higher HCQ exposure. The MTD was found to be $600 \mathrm{mg} \mathrm{HCQ}$ po qd as sustained hematological toxicity prevented further dose escalation. When comparing the clinical outcome to historical data, the OS was not significantly different. HCQ was preferred over CQ based 
on a more favorable toxicity profile with a lower risk of retinopathy and cardiotoxicity. This is reflected in the significantly higher MTD in comparison to the MTD of $200 \mathrm{mg}$ CQ found in this study. However, the authors could not exclude the possibility that the combination of CQ with radiotherapy and TMZ would have been more potent, as CQ crosses the blood-brain barrier more easily and can potentially accumulate in brain tissue to a higher level than the blood plasma concentration ${ }^{30}$.

In addition to glioma, CQ is currently being investigated in a wide range of cancer types, with 3 studies currently recruiting including a large phase III trial in glioma (NCT03243461) (clinicaltrials.gov). In a phase I study, the combination of $300 \mathrm{mg}$ CQ per day with gemcitabine in patients with metastatic or unresectable pancreatic cancer was well-tolerated ${ }^{31}$. A recent randomized window-of-opportunity trial in breast cancer patients has evaluated single-agent $500 \mathrm{mg}$ CQ per day in a preoperative setting ${ }^{32}$. No significant effect of CQ on tumor proliferation was established. Nearly $15 \%$ of patients discontinued treatment due to adverse events, mainly nausea and/or abdominal cramps.

Induction of autophagy by radiation and TMZ results in radioresistance of glioma stem cells, which can be inhibited with CQ ${ }^{13,21,33-35}$. Several studies have demonstrated that inhibition of autophagy caused by radiotherapy and TMZ can ameliorate apoptosis in GBM cells and thereby increase therapeutic outcome ${ }^{20,36-38}$. There is evidence that $\mathrm{CQ}$ exerts its therapeutic effects also by autophagy-independent mechanisms such as promotion of lysosomal membrane permeabilization inducing apoptosis ${ }^{39,40}$ and tumor vessel normalization ${ }^{23}$. Autophagy has been described as an important regulator of cellular immune response, inhibiting the tumor-specific immune response through suppression of ATP release attracting dendritic cells and T-lymphocytes ${ }^{41,42}$. Moreover, it can lead to accumulation TLR4 (toll like receptor 4) on the cell surface, thereby increasing the tumor-specific immune response $\mathrm{e}^{43,44}$.

EGFRVIII is a potential predictive biomarker for the addition of CQ to concurrent chemoradiation. Even though a formal statistical analysis was not possible, Fig. 2 shows that CQ may increase OS in EGFRvIll-positive tumors. Overexpression of EGFRvIII has been shown to increase cell proliferation and survival under stress conditions such as hypoxia or nutrient deprivation by an increase in autophagic activity ${ }^{9,45,46}$. A retrospective analysis of patients with a GBM treated with radiotherapy and carmustine demonstrated that the addition of CQ improved OS from 5 to 10 months in the EGFRvIIInegative GBM and improved survival from 3 to 15 months in the EGFRvIll-positive $\mathrm{GBM}^{10}$.

Although the pharmacokinetic properties of $C Q$ alone are known, the pharmacokinetic properties of $C Q$ in combination with $T M Z$ have not yet been investigated. The required dose for autophagy inhibition has not yet been established. The trough level was chosen as it is directly measurable and the most reliable assessment of the CQ 
plasma concentration. The $\mathrm{CQ}$ plasma $\mathrm{T}_{1 / 2}$ is ca 2 weeks, meaning a steady-state plasma concentration was expected to be reached in the second week of chemoradiation. In this trial, a near steady-state was observed after 2 weeks (data not shown). Rosenfeld et al have previously demonstrated a dose-dependent increase in autophagy inhibition for $\mathrm{HCQ}^{29}$. Although we did not reach the planned $600 \mathrm{mg} \mathrm{CQ}$ dose level in this study, pharmacokinetic studies have shown that patients will reach a steady-state concentration of approximately $10 \mu \mathrm{M} \mathrm{CQ}$ with a daily intake of $250 \mathrm{mg}$, which has been shown to be an effective dose for autophagy inhibition ${ }^{36,47}$. Yet other studies suggest that higher doses of $C Q$ are required for a therapeutic effect ${ }^{48,49}$. This possibly explains an apparent lack of effect on OS in our overall patient cohort.

This study has several limitations. Although this study's traditional $3+3$ design is considered safe, it does have several disadvantages, e.g., the low likelihood of selecting the true MTD due to pre-defined dose levels, uncertainty in detecting all DLTs because of low patient numbers and no option to re-escalate the CQ dose. CQ concentrations vary significantly between different types of tissue, e.g., $\mathrm{CQ}$ accumulates in red blood cells, and the concentration within red blood cells may be $>4-10 \times$ higher than the plasma concentration ${ }^{50}$. However, the serum fraction of CQ is in direct contact with the possible target tissue. Therefore, we hypothesize this concentration to be more relevant with regards to both the effects and side effects of $C Q$, and therefore chose to quantify CQ based on serum concentration. This should be further investigated in future trials. CQ was initiated one week before the start of chemo- and radiotherapy in order to reach a steady-state early into treatment. However, it is unclear if a steady state is necessary to reach optimal autophagy inhibition. Unlike previous clinical trials ${ }^{24,29}$, CQ was stopped at the end of concurrent chemoradiation in order to avoid potential long-term toxicity caused by CQ's extensive plasma terminal elimination half-life of 40 $-60 \mathrm{~d}$ and its active metabolites. We, however, cannot exclude that CQ concurrent to the adjuvant cycles of TMZ is a more effective combination. Further research into the timing of adjuvant CQ at the time of chemotherapy resistance is necessary ${ }^{19}$. LC3B-II within peripheral blood mononuclear cells currently serves as a surrogate to measure the inhibitory effect of CQ in tumor cells $s^{51}$. There currently is no technique available to directly measure autophagy inhibition within the tumor. Further development of a biomarker or tracer to evaluate autophagy inhibition is highly desirable to evaluate the clinical effect in vivo.

In conclusion, this study has shown that despite CQ's well-known toxicity profile, it may still elicit significant adverse effects in combination with TMZ and radiotherapy in doses commonly described for rheumatoid arthritis. It is anticipated that more potent and less toxic autophagy inhibitors such as Lys05 or Verteporfin will become clinically available throughout the years to come, but these are currently still under investigation in preclinical models. Combination treatment with autophagy inhibitors has the potential to become the new standard of care for GBM as it may significantly impact survival, especially for a subset of patients. 


\section{Acknowledgements}

The authors sincerely thank Monique Bessems, Nadja Drummen, John Paulissen and Claudia Offermans for their support with data collection, patient inclusion and patient guidance. Moreover, the authors would like to thank the members of the Data Safety Monitoring Board, Filip Y.F.L. De Vos MD, PhD, prof. Daan J. Touw, Pharm D, PhD, Ruud M.A. Houben, MSc, Iverna Nijsten, MSc, and Kim M. Smits, PhD for their expertise and recommendations. The study protocol was written during the 16th joint ECCO-AACR-EORTC-ESMO Workshop Methods in Clinical Cancer Research Flims, Switzerland, 21-27.6.2014 
1. de Robles $P$, Fiest $K M$, Frolkis $A D$, et al. The worldwide incidence and prevalence of primary brain tumors: a systematic review and meta-analysis. Neuro, Oncol. 2015;17(6):776-783.

2. Stupp R, Hegi ME, Mason WP, et al. Effects of radiotherapy with concomitant and adjuvant temozolomide versus radiotherapy alone on survival in glioblastoma in a randomised phase III study: 5-year analysis of the EORTC-NCIC trial. Lancet Oncol. 2009;10(5):459-466.

3. Weller $M$, van den Bent $M$, Tonn JC, et al. European Association for NeuroOncology (EANO) guideline on the diagnosis and treatment of adult astrocytic and oligodendroglial gliomas. Lancet, Oncol. 2017;18(6):e315-e329.

4. Patel AP, Tirosh I, Trombetta JJ, et al. Single-cell RNA-seq highlights intratumoral heterogeneity in primary glioblastoma. Science (New York, NY). 2014;344(6190):1396-1401.

5. Louis DN, Perry A, Reifenberger G, et al. The 2016 World Health Organization Classification of Tumors of the Central Nervous System: a summary. Acta neuropathologica. 2016;131(6):803-820.

6. Maire $\mathrm{CL}$, Ligon $\mathrm{KL}$. Molecular pathologic diagnosis of epidermal growth factor receptor. Neuro-oncology. 2014;16 Suppl 8:viii1-6.

7. Brennan CW, Verhaak Rg Fau - McKenna A, McKenna A Fau - Campos B, et al. The somatic genomic landscape of glioblastoma. Cell. 2013;155(2):462-477.

8. Shinojima N, Tada K, Shiraishi S, et al. Prognostic value of epidermal growth factor receptor in patients with glioblastoma multiforme. Cancer Res. 2003;63(20): 6962-6970.

9. Theys J, Jutten B, Dubois L, et al. The deletion mutant EGFRvIll significantly contributes to stress resistance typical for the tumour microenvironment. Radiother Oncol. 2009;92(3):399-404.

10. Jutten B, Keulers TG, Peeters HJM, et al. EGFRvIll expression triggers a metabolic dependency and therapeutic vulnerability sensitive to autophagy inhibition. Autophagy. 2018;14(2):283-295.

11. Wouters BG, van den Beucken $T$, Magagnin MG, Lambin P, Koumenis C. Targeting hypoxia tolerance in cancer. Drug Resist Updat. 2004;7(1):25-40.

12. Mizushima N. The pleiotropic role of autophagy: from protein metabolism to bactericide. Cell death and differentiation. 2005;12 Suppl 2:1535-1541.

13. Kanzawa T, Germano IM, Komata $T$, Ito $H$, Kondo $Y$, Kondo $S$. Role of autophagy in temozolomide-induced cytotoxicity for malignant glioma cells. Cell death and differentiation. 2004;11(4):448-457.

14. Paglin S, Hollister T, Delohery T, et al. A novel response of cancer cells to radiation involves autophagy and formation of acidic vesicles. Cancer Res. 2001; 61(2): 439-444.

15. Ito H, Daido S, Kanzawa T, Kondo S, Kondo Y. Radiation-induced autophagy is associated with LC3 and its inhibition sensitizes malignant glioma cells. International journal of oncology. 2005;26(5):1401-1410. 
16. Yao KC, Komata T, Kondo Y, Kanzawa T, Kondo S, Germano IM. Molecular response of human glioblastoma multiforme cells to ionizing radiation: cell cycle arrest, modulation of the expression of cyclin-dependent kinase inhibitors, and autophagy. Journal of neurosurgery. 2003;98(2):378-384.

17. Rouschop KM, van den Beucken $T$, Dubois $L$, et al. The unfolded protein response protects human tumor cells during hypoxia through regulation of the autophagy genes MAP1LC3B and ATG5. J Clin Invest. 2010;120(1):127-141.

18. Savarino A, Boelaert JR, Cassone A, Majori G, Cauda R. Effects of chloroquine on viral infections: an old drug against today's diseases? Lancet Infect Dis. 2003;3(11):722-727.

19. Golden EB, Cho HY, Jahanian A, et al. Chloroquine enhances temozolomide cytotoxicity in malignant gliomas by blocking autophagy. Neurosurg Focus. 2014;37(6):E12.

20. Hori YS, Hosoda R, Akiyama Y, et al. Chloroquine potentiates temozolomide cytotoxicity by inhibiting mitochondrial autophagy in glioma cells. Journal of neuro-oncology. 2015;122(1):11-20.

21. Firat E, Weyerbrock A, Gaedicke S, Grosu AL, Niedermann G. Chloroquine or chloroquine-PI3K/Akt pathway inhibitor combinations strongly promote gamma-irradiation-induced cell death in primary stem-like glioma cells. PLoS One. 2012;7(10):e47357.

22. Rouschop KM, Ramaekers $\mathrm{CH}$, Schaaf $\mathrm{MB}$, et al. Autophagy is required during cycling hypoxia to lower production of reactive oxygen species. Radiother Oncol. 2009;92(3):411-416.

23. Maes $H$, Kuchnio $A$, Peric $A$, et al. Tumor vessel normalization by chloroquine independent of autophagy. Cancer Cell. 2014;26(2):190-206.

24. Sotelo J, Briceno E, Lopez-Gonzalez MA. Adding chloroquine to conventional treatment for glioblastoma multiforme: a randomized, double-blind, placebocontrolled trial. Ann Intern Med. 2006;144(5):337-343.

25. Marmor MF, Kellner U, Lai TY, Lyons JS, Mieler WF. Revised recommendations on screening for chloroquine and hydroxychloroquine retinopathy. Ophthalmology. 2011;118(2):415-422.

26. White NJ. Cardiotoxicity of antimalarial drugs. Lancet Infect, Dis. 2007;7(8):549558.

27. Bilger A, Bittner MI, Grosu AL, et al. FET-PET-based reirradiation and chloroquine in patients with recurrent glioblastoma : First tolerability and feasibility results. Strahlentherapie und Onkologie : Organ der Deutschen Rontgengesellschaft [et al]. 2014;190(10):957-961.

28. Briceno E, Calderon A, Sotelo J. Institutional experience with chloroquine as an adjuvant to the therapy for glioblastoma multiforme. Surg Neurol. 2007;67(4):388391.

29. Rosenfeld MR, Ye X, Supko JG, et al. A phase I/II trial of hydroxychloroquine in conjunction with radiation therapy and concurrent and adjuvant temozolomide in patients with newly diagnosed glioblastoma multiforme. Autophagy. 2014;10(8):1359-1368. 
30. Adelusi SA, Salako LA. Tissue and blood concentrations of chloroquine following chronic administration in the rat. The Journal of pharmacy and pharmacology. 1982;34(11):733-735.

31. Samaras P, Tusup M, Nguyen-Kim TDL, et al. Phase I study of a chloroquinegemcitabine combination in patients with metastatic or unresectable pancreatic cancer. Cancer Chemother, Pharmacol. 2017;80(5):1005-1012.

32. Arnaout A, Robertson SJ, Pond GR, et al. A randomized, double-blind, window of opportunity trial evaluating the effects of chloroquine in breast cancer patients. Breast Cancer Res Treat. 2019;178(2):327-335.

33. Sui $X$, Chen $R$, Wang $Z$, et al. Autophagy and chemotherapy resistance: a promising therapeutic target for cancer treatment. Cell death \& disease. 2013;4:e838.

34. Wurstle S, Schneider F, Ringel F, et al. Temozolomide induces autophagy in primary and established glioblastoma cells in an EGFR independent manner. Oncology letters. 2017;14(1):322-328.

35. Ye $\mathrm{H}$, Chen $\mathrm{M}$, Cao F, Huang $\mathrm{H}$, Zhan R, Zheng X. Chloroquine, an autophagy inhibitor, potentiates the radiosensitivity of glioma initiating cells by inhibiting autophagy and activating apoptosis. BMC neurology. 2016;16(1):178.

36. Lee SW, Kim HK, Lee NH, et al. The synergistic effect of combination temozolomide and chloroquine treatment is dependent on autophagy formation and p53 status in glioma cells. Cancer Lett. 2015;360(2):195-204.

37. Zanotto-Filho A, Braganhol E, Klafke K, et al. Autophagy inhibition improves the efficacy of curcumin/temozolomide combination therapy in glioblastomas. Cancer Lett. 2015;358(2):220-231.

38. Lin CJ, Lee CC, Shih YL, et al. Inhibition of mitochondria- and endoplasmic reticulum stress-mediated autophagy augments temozolomide-induced apoptosis in glioma cells. PLoS One. 2012;7(6):e38706.

39. Enzenmuller S, Gonzalez P, Debatin KM, Fulda S. Chloroquine overcomes resistance of lung carcinoma cells to the dual PI3K/mTOR inhibitor PI103 by lysosome-mediated apoptosis. Anti-cancer drugs. 2013;24(1):14-19.

40. Maycotte $P$, Aryal S, Cummings CT, Thorburn J, Morgan MJ, Thorburn A. Chloroquine sensitizes breast cancer cells to chemotherapy independent of autophagy. Autophagy. 2012;8(2):200-212.

41. Michaud M, Xie X, Bravo-San Pedro JM, Zitvogel L, White E, Kroemer G. An autophagy-dependent anticancer immune response determines the efficacy of melanoma chemotherapy. Oncoimmunology. 2014;3(7):e944047.

42. Ko A, Kanehisa A, Martins I, et al. Autophagy inhibition radiosensitizes in vitro, yet reduces radioresponses in vivo due to deficient immunogenic signalling. Cell death and differentiation. 2014;21(1):92-99.

43. Husebye $\mathrm{H}$, Halaas $\mathrm{O}$, Stenmark $\mathrm{H}$, et al. Endocytic pathways regulate Toll-like receptor 4 signaling and link innate and adaptive immunity. The EMBO journal. 2006;25(4):683-692.

44. Apetoh L, Ghiringhelli F, Tesniere A, et al. Toll-like receptor 4-dependent contribution of the immune system to anticancer chemotherapy and radiotherapy. Nature medicine. 2007;13(9):1050-1059. 
45. Golding SE, Morgan RN, Adams BR, Hawkins AJ, Povirk LF, Valerie K. Prosurvival AKT and ERK signaling from EGFR and mutant EGFRvIll enhances DNA double-strand break repair in human glioma cells. Cancer biology \& therapy. 2009;8(8):730-738.

46. Weppler SA, Li Y, Dubois $L$, et al. Expression of EGFR variant vlll promotes both radiation resistance and hypoxia tolerance. Radiother Oncol. 2007;83(3):333-339.

47. Augustijns $P$, Geusens $P$, Verbeke N. Chloroquine levels in blood during chronic treatment of patients with rheumatoid arthritis. Eur J Clin Pharmacol. 1992;42(4):429-433.

48. Weyerhauser P, Kantelhardt SR, Kim EL. Re-purposing Chloroquine for Glioblastoma: Potential Merits and Confounding Variables. Front, Oncol. 2018;27(8):335.

49. Pascolo S. Time to use a dose of Chloroquine as an adjuvant to anti-cancer chemotherapies. Eur, J Pharmacol. 2016;15(771):139-144.

50. Titus EO. Recent developments in the understanding of the pharmacokinetics and mechanism of action of chloroquine. Ther Drug Monit. 1989;11(4):369-379.

51. Mizushima N, Yamamoto A Fau - Matsui M, Matsui M Fau - Yoshimori T, Yoshimori T Fau - Ohsumi Y, Ohsumi Y. In vivo analysis of autophagy in response to nutrient starvation using transgenic mice expressing a fluorescent autophagosome marker. Molecular biology of the cell. 2004;15(3):1101-1111.

52. Le Tourneau C, Lee JJ, Siu LL. Dose escalation methods in phase I cancer clinical trials. Journal of the National Cancer Institute. 2009;101(10):708-720.

53. European Medicines Agency (EMA). Guideline on bioanalytical method validation 2019; https://www.ema.europa.eu/en/documents/scientificguideline/guideline-bioanalytical-method-validation_en.pdf.

54. U.S. Department of Health and Human Services Food and Drug Administration. Bioanalytical Method Validation. Guidance for Industry. 2018; https://www.fda.gov/files/drugs/published/Bioanalytical-Method-ValidationGuidance-for-Industry.pdf. 
Chapter 7 - General Discussion 


\section{General discussion}

The general aim of the research work presented in this thesis was to investigate the potential gain of technological innovations in glioblastoma (GBM) diagnosis and therapy that could be implemented in the near future to improve the prognosis of patients with this disease. This work was divided into three parts: 1) decision support systems, 2) ultra-high field MRI, 3) autophagy inhibition, for which the following specific objectives had been defined:

1. Review the current and novel diagnostic imaging methods and techniques used for the prediction of treatment response and the optimization of decision support systems to improve outcome prediction in patients with a GBM (Chapter 2 and 3 ).

2. Investigate the technical feasibility of incorporating 7 Tesla MRI into neurosurgical navigation and radiation treatment planning systems for patients with GBM (Chapter 4 and 5).

3. Determine the recommended phase II dose for chloroquine (CQ) in combination with concurrent radiotherapy with temozolomide (TMZ) in patients with a newly diagnosed GBM (Chapter 6).

This chapter presents a critical appraisal of the topics investigated. The three parts of this thesis are summarized and put into the current clinical perspective, after which future perspectives are discussed.

\section{Decision support systems}

Chapter 2 provides a comprehensive overview of the currently available techniques that capture tumor heterogeneity of GBM in a non-invasive way and can be integrated into decision support systems. This chapter reviews imaging techniques such as MR imaging, nuclear imaging and liquid biopsies and novel integrated approaches such as radiogenomics and radiomics that allow to both monitor and predict the treatment response. This chapter also introduces the "Non-invasive Glioblastoma Testing" (NIGT) platform. With the diagnostic techniques integrated in the NIGT platform information on the tumor as a whole, including driver mutations and the tumor microenvironment, can be captured in a non-invasive manner. Histopathological and molecular analysis of tissue samples taken by a biopsy or resection is currently the golden standard for the diagnosis of GBM. However, GBM demonstrate a large amount of intratumoral heterogeneity that often cannot be fully captured with these types of analyses, especially when only a biopsy is taken. This intratumoral heterogeneity is one of the main challenges for GBM treatment as clones within the tumor may demonstrate a different amount of sensitivity to both radiation treatment and chemotherapy, ultimately resulting in treatment resistance. The NIGT platform will enable physicians to select the optimal treatment both at the stage of diagnosis and recurrence without the need for repeated tissue verification. 
One of NIGT's approaches includes radiomics, which is the identification of noninvasive imaging biomarkers by high-throughput data mining of large amounts of quantifiable features from images based on intensity, shape, texture and wavelets ${ }^{1,2}$. In Chapter 3 a radiomics study in patients with GBM is presented that investigates the hypothesis that CT-scans may hold prognostic information regarding OS that is visually undetectable to the human eye. In this study three models (clinical, volumebased and radiomics) were trained to divide the population into three risk groups: the best prognosis (25\%), the worst prognosis (25\%) and the remainder in the middle. These models were based on clinical data and pre-treatment CT-images obtained from three independent cohorts of 93, 62 and 63 patients who were diagnosed with a GBM via biopsy and treated with radiotherapy +/- TMZ between 2004 and 2015 in three institutes. The predictive performance of each of the models was estimated using the internal-external validation method, with the data of each centre being left out in turn. Moreover, for the prognostic signature the global model coefficients were determined using all three datasets pooled. The simple clinical model based on age and WHO performance status demonstrated a moderate discriminative clinical signature with an apparent c-index 0.66. The RPS model resulted in a comparable apparent c-index of 0.66 , whereas the VPS returned a c-index of 0.55 , inferior to the other two models.

The presented study was the first to investigate OS predication with CT-based radiomics in GBM. One of the major strengths of this study is the attempt to validate the proposed models in two external datasets, whereas other radiomics studies in GBM often did not include external validation of their results or only applied internal validation. External validation is the preferred method as the data is acquired independently at two other treatment centres, which supports the validation ${ }^{3}$. Potential explanations why both the clinical and radiomics models are only moderately discriminative include the retrospective collection of data, a relatively small patient cohort, missing data (i.e. clinical characteristics and molecular markers), inter- and intraobserver variability due to manual tumor delineation on MR and CT imaging, and the fact that the CT scans were acquired after biopsies. As a result of these biopsies blood remnants may be present, which could potentially interfere with the radiomics analysis. Our study was reported according to the 'Transparent Reporting' criteria of a multi-variable prediction model for Individual Prognosis Or Diagnosis (TRIPOD) initiative 4 . However, when comparing this study to the recently published radiomics quality score several additional limitations can be noted 5 . These include the absence of multiple delineations, a phantom study performed on all applied CT-scanners, the analysis of temporal variabilities through imaging at multiple time points and a cost-effectiveness analysis. Another major issue may be the lack of standardization in imaging protocols between the three participating institutes. Although imaging characteristics were broadly comparable, there were small differences in image resolution, exposure factors, back projection kernel, slice 
thickness and manufacturers of CT-scanners used. A larger prospectively collected dataset, including standardized homogenized imaging protocols, is required to improve the performance of radiomics models. Although there may be potential prognostic information with regard to OS hidden in images that can be disclosed through radiomics, the above limitations and issues need to be addressed in future research.

As MRI provides superior soft-tissue contrast it is the preferred imaging modality for diagnosis, treatment planning and follow-up in patients with GBM. In the past few years multiple radiomics studies have been conducted on $\mathrm{MRI}^{6-11}$. One of the major advantages of MRI over CT is the widespread availability of imaging series from baseline and 3-monthly follow-up until death. Moreover, most European institutions adhere to the EANO guideline which recommends $T_{2}$-weighted and fluid-attenuated inversion recovery sequences, and $T_{1}$-weighted scans acquired before and after gadolinium-based contrast enhancement ${ }^{12}$. Several studies have identified prognostic or predictive imaging biomarkers on MRI in glioma that are associated with survival, histology, tumor grade or molecular characteristics ${ }^{6-11}$. Major limitations of these studies are that MRI data was acquired on a variety of scanners with various image acquisition protocols and pulse sequence parameters, patient cohorts are relatively small and external validation on large independent datasets is often lacking. The absence of standardization of image acquisition results in the need to pre-process the imaging data prior to radiomics analysis. Methods such as intensity normalization (e.g. Gaussian and Z-score normalisation) and voxel re-slicing potentially introduce a loss of information ${ }^{13,14}$. These limitations led us to investigate the potential of $\mathrm{CT}$ imaging for radiomics modelling. As Hounsfield Units are obtained from a linear transformation of the measured attenuation coefficients which is based on the densities of air and water, the CT images analysed in the presented study did not require normalization before image analysis.

The ultimate objective of the NIGT platform, including future radiomics models, is to create personalized medicine for patients with a GBM. Personalized medicine is the application of information about a person's clinical, genetic, genomic and environmental characteristics to maximize the patient's changes of better prevention, diagnosis or treatment15. This information can be gathered into decision support systems, which allow clinicians to appreciate the available factors and determine the optimal cancer treatment taking into account toxicity, tumor control rate, quality of life and cost effectiveness ${ }^{16}$. Until a few years ago personalized medicine was only scarcely applied for the treatment of glioma and was predominantly based on pathological or clinical factors such as tumor grade and performance status. Currently, with the discovery of the prognostic and/or predictive value of molecular markers such as MGMT promotor methylation, mutations in IDH 1 and 2 and $1 p / 19 q$ co-deletion patients with the same histological tumor are treated differently ${ }^{17,18}$. Several studies have demonstrated the clinical relevance of MGMT promotor 
methylation as prognostically favourable in grade III gliomas receiving radiotherapy and/or chemotherapy ${ }^{19,20}$. Moreover, this molecular marker has both an important prognostic and predictive value in glioblastoma ${ }^{21-23}$. As a result, some countries now adapt treatment plans based on MGMT methylation status for specific subset of glioma patients, e.g. frail or elderly patients. Co-deletion of $1 p / 19 q$ is prognostically favourable in grade II and III gliomas and is also predictive for the benefit of procarbazine, lomustine and vincristine (PCV) chemotherapy regimen in grade III glioma ${ }^{24,25}$. And finally, mutations in $I D H-1$ and 2 genes are prognostically favourable in grade II - IV glioma ${ }^{19,26}$, whereas patients with a grade III glioma with an IDH wildtype have a worse prognosis than patients with a GBM with an IDH mutation ${ }^{27}$. As a result, grade III IDH wildtype astrocytomas are nowadays treated as a molecular continuum of GBM. It is noteworthy that detection of $I D H$ mutation often coincides with MGMT promotor methylation ${ }^{28}$.

However, the major challenge for the development of any decision support system in glioma is the heterogeneity of the cohorts on which the models are based. In order to fully implement decision support systems in the treatment of glioma a tremendous volume of high-quality, well-annotated data needs to be collected. This would require at least a national collaboration in e.g. the Dutch Neuro-Oncology Society (Landelijke Werkgroep Neuro-Oncologie) through which standardized prospective data collection protocols (umbrella protocols) are developed. A meticulous prospective registration of clinical characteristics, histology and molecular analysis, response to treatment, tumor control and toxicity while collecting this data, is vital. This would also require the development of a uniform ontology and continuous quality assurance. Moreover, the standardization of the criteria for image acquisition and a generally accepted feature definition is essential for any system to be usable in more than one clinic. Examples of standardized image acquisition for CT include an agreement on e.g. slice thickness, the use of contrast-enhancement, back projection kernel, nominal tube potential. Finally, methods are needed to overcome the obstacles that are related to world-wide data sharing, such as data-sharing technology and legal and privacy considerations $s^{5,29,30}$. This will allow for the necessary external validation and further development of the constructed models. In order to be able to clinically implement such a model to determine if a patient with a GBM would benefit from extensive treatment in terms of survival a model would require a very high positive and negative predictive value as it could result in patients being denied radical treatment based on its results. Overcoming these hurdles is considered the most important first steps towards the development of any more universally usable decision support system. 


\section{Ultra-high field MRI}

Ultra-high field MRI provides an increased signal-to-noise-ratio (SNR) and hence allows for higher spatial resolution relative to high-field $\mathrm{MR}^{13,32}$. Chapter 4 and 5 discuss the necessary steps taken in preparation for a prospective trial investigating the value of $7 \mathrm{~T} \mathrm{MRI} \mathrm{in} \mathrm{the} \mathrm{delineation} \mathrm{of} \mathrm{the} \mathrm{GTV} \mathrm{in} \mathrm{patients} \mathrm{with} \mathrm{a} \mathrm{newly} \mathrm{diagnosed}$ GBM and the clinical implementation of $7 \mathrm{~T} \mathrm{MRI}$ in a radiation therapy setting. It includes both a qualitative and quantitative analysis of $7 \mathrm{~T}$ MR images.

The main hypothesis of the clinical trial was that $7 \mathrm{~T} \mathrm{MRI}$ allows for an improved delineation of GBM cells outside the contrast-enhanced volume due to the visualisation of microvascularisation. This hypothesis was based on three observations. Firstly, 7 $\mathrm{T} \mathrm{T2}{ }^{*}-\mathrm{w}$ images can better depict increased microvascularity in high-grade glioma when compared to $1.5 \mathrm{~T}^{33,34}$. Secondly, the WHO tumor grade can be predicted using susceptibility weighted imaging (SWI) at $7 \mathrm{~T}$ for visualization of tumor microvascularity and the tumor grade is associated with the number and size of foci of microvascularity within the tumor bed at UHF GRE MRI ${ }^{35,36}$. Thirdly, differences in magnetic susceptibility effects associated with deoxyhemoglobin in venous blood, differences in myelin binding fractions of water, and iron-containing molecules such as hemosiderin allow for an overall high resolution of the brain microvasculature on $\mathrm{UHF} \mathrm{MRI}^{37}$. These observations are very relevant as an increase in angiogenesis is one of the key features of GBM and microvasculature can be used as an imaging surrogate of angiogenesis ${ }^{38}$.

The preparatory study described in Chapter 4 was divided into two parts: (1) a pilot study involving healthy volunteers in which both 7T MR image acquisition and image quality were analysed and (2) a phantom study which analysed the geometrical image distortion in 7 T MR images and compared this to CT images. An image transfer- and processing workflow for the application of $7 \mathrm{~T} \mathrm{MR}$ images was also developed.7T MR sequences were selected to highlight differences in tissue contrast and visualize microvascularization and included multi-echo gradient echo (GRE), magnetization-prepared rapid gradient-echo (MP2RAGE), T2-sampling perfection with application optimized contrasts using different flip-angle evolution (T2-SPACE), and sampling perfection with application optimized contrasts using different flipangle evolution FLAIR (SPACEFLAIR). For the phantom study an anthropomorphic skull phantom was used. The pilot study showed that the scanning sessions were well tolerated by all three volunteers and only minor vestibular effects were reported. Images acquired with MP2RAGE, T2-SPACE, and SPACE-FLAIR sequences all displayed good to excellent SNR and visualization of the frontal and parietal lobes, ventricles and CSF. There was a significant decrease in image quality near the skull base which resulted in a non-diagnostic to marginal image quality of the surrounding tissue (frontobasal and temporal lobes). However, ghosting and flow artefacts were present in the images. The phantom study demonstrated that geometrical distortion was more pronounced in MR images than in CT images and increased exponentially with 
increasing distances from the imaging isocenter. In addition, geometrical distortion differed between MR pulse sequences. This could be attributed to differences in read-out bandwidth. The main limitations of this study include a limited number of healthy volunteers, only 4 clinically optimized 7T MR pulse sequences with fixed scan parameters were investigated, the anthropomorphic skull phantom only allowed for assessment of the system-related geometrical distortion rather than also the object-related geometrical distortion, and geometrical distortion of the $7 \mathrm{~T} \mathrm{MRI}$ in comparison to lower field strengths was not investigated. However, a comparison with $3 \mathrm{~T} \mathrm{MRI}$ and CT is discussed in Chapter 5 . In conclusion, the integration of highquality and geometrically reliable $7 \mathrm{~T}$ anatomical MR images into neurosurgical navigation and RTP software is technically feasible and safe for the system and pulse sequences studied. Although the development of UHF MRI is quickly moving forward several technical issues still need to be resolved before clinical implementation, such as signal and susceptibility artefacts near air-tissue interfaces and geometrical distortion by increased static magnetic field $\left(B_{0}\right)$ inhomogeneity at larger distances from the imaging isocentre. Future studies should also focus on improving image quality of UHF MRI by reducing inhomogeneity of the transmit $B_{1}$-field, reducing the specific absorption rate and decreasing tissue susceptibility differences and chemical shift effects.

So far, only one other group has investigated $7 \mathrm{~T}$ MRI for radiation treatment planning in $\mathrm{GBM}^{39}$. They compared a 7 T FLAIR sequence with FLAIR imaging at $3 \mathrm{~T} \mathrm{MRI}$ and also hypothesized that $7 \mathrm{~T}$ MRI would yield a higher spatial resolution at equally high image quality compared to $3 \mathrm{~T} \mathrm{MRI}$, resulting in an altered GTV. Based on their analysis, which included 15 GBM patients, they demonstrated that 7 T FLAIR yields significantly higher SNR in white matter, resulting in an improved depiction of white matter tracts. Moreover, they reported an increase in contrast between grey matter and white matter, whereas the contrast between peritumoral edema and white matter was significantly decreased. The 7 T FLAIR-based GTVs were significantly smaller when compared to 3 T FLAIR imaging $(79.09 \mathrm{ml} v$ s. $76.58 \mathrm{ml}, p=0.03)$. The higher SNR in white matter and improved contrast between grey and white matter suggested better visibility of anatomical borders, which could improve delineation of essential OARs such as the chiasm or hippocampus ${ }^{40}$. Similar to GBM, the research for the clinical application of UHF MRI in other neuro-oncological disorders is limited with only a handful of studies investigating $7 \mathrm{~T} \mathrm{MRI}$ in pituitary adenoma and meningioma ${ }^{41-44}$.

In Chapter 5 the geometrical accuracy of CT, 3 T and 7 T MR imaging for highprecision radiotherapy in the brain is characterized. With the introduction of stereotactic radiotherapy and more recently proton therapy in the Netherlands, high-quality images for radiation therapy planning with small PTV margins are becoming increasingly important. Accurate image guidance for treatment planning and treatment delivery is essential as relatively small inaccuracies can result in 
significant underdosage of the target volume or a high dose in an OAR with reported dose variations of 5-10\% within $1 \mathrm{~mm}^{45}$. System- and patient-related geometrical distortions are increasingly observed with higher magnetic field strength and can be induced by imperfections in the hardware $\left(B_{0}\right.$-inhomogeneity, gradient field nonlinearity and eddy currents), applied gradient pulse scheme and parameter settings or patient related geometrical distortion due to organ motion, chemical shift and magnetic susceptibility differences ${ }^{46,47}$. Geometrical distortion generally increases with an increasing distance from the imaging isocentre of the MR scanner and system-related geometrical distortion can at least be in part corrected for by distortion correction software on the MR scanner. As patient-related geometrical distortion is patient/tissue specific, a general solution cannot be applied. However, an increase in receiver bandwidth or adjustment of imaging echo time may reduce chemical shifts significantly ${ }^{48,49}$.

In the study described in Chapter 5, three hypotheses were tested: 1) acceptable submillimetre spatial accuracy can be achieved with $7 \mathrm{~T} \mathrm{MRI}, 2)$ no significant differences in geometrical distortion is present between different $7 \mathrm{~T}$ sequences, 3 ) the tolerated level of geometrical distortion in $7 \mathrm{~T}$ MRI is not significantly different from system-related geometrical distortion on $3 \mathrm{~T}$ MRI. $7 \mathrm{~T}$ MRI pulse sequences were duplicated from the preparatory study described in Chapter 4 and included GRE, MP2RAGE, T2-SPACE and SPACEFLAIR. For comparison 3 T MRI sequences with equivalent pulse schemes included turbo field echo (3D TFE), volume isotropic turbo spinecho (T2-VISTA) with and without FLAIR, and fast field gradient echo (T1-FFE). The same anthropomorphic skull phantom as used in Chapter 4 was applied in this study. Geometrical distortion was assessed with three different methods. Significant differences in geometrical distortion were detected between all equivalent sequences on $7 \mathrm{~T}$ and $3 \mathrm{~T}$ MR systems. Notably an anisotropic distribution which particularly affected the anterior-superior section was identified. Moreover, geometrical distortion increased with increasing distance from the isocentre. At the clinically relevant diameter of a spherical volume of $45 \mathrm{~cm}$ the geometrical distortion remained below the tolerance level of $1 \mathrm{~mm}$ and no significant difference was observed between equivalent $3 \mathrm{~T}$ and $7 \mathrm{~T}$ sequences. This study demonstrates that in central brain regions the $7 \mathrm{~T}$ MRI sequences investigated can be applied for high-precision radiotherapy treatment planning as geometrical distortion $\leq 1 \mathrm{~mm}$ could be assured near the magnetic isocentre. For targets located in the brain peripheral regions, the geometrical distortion should be incorporated in the PTV margins to correct for treatment uncertainties. Several limitations should be noted and include the fact that only the system-related geometrical distortion could be evaluated with the phantom, points of interest were manually reconstructed and could have introduced errors and MR sequences were optimized for clinical (diagnostic) purposes, in contrast to the high geometrical accuracy protocols required for radiation treatment planning. Our findings were comparable to those found by other investigators ${ }^{50,51}$. However, at a very large and for neuro-oncological purposes clinically less relevant distances from 
the magnetic isocentre other investigators noted larger geometrical distortion of up to several centimetres ${ }^{52,53}$. A study assessing geometrical distortion with regard to image guidance in neurosurgery found the hardware-related geometrical distortions at $7 \mathrm{~T} \mathrm{MRI}$ are relatively small (up to $1.6 \mathrm{~mm}$ ) and integration of $7 \mathrm{~T} \mathrm{MRI} \mathrm{into} \mathrm{image-}$ guided applications was feasible ${ }^{54}$. Recently, a 3D multimodality phantom system for the quantitative assessment of geometric accuracy of MRI and CT images used for radiation treatment planning was presented 55 . With this system image distortion can be evaluated using a centroid algorithm that automatically identifies the center of each 3D-intersection and then calculates the deviations, thereby reducing errors introduced by manual reconstruction of the points of interest. Only geometrical distortion for CT, 1.5 T and $3 \mathrm{~T}$ MRI sequences was evaluated and for $3 \mathrm{~T} \mathrm{MRI}$ geometrical distortion was comparable to the results found in our study. Similar, geometrical distortion in the $y$-direction was larger caused by frequency encoding in this direction.

To conclude, the study in Chapter 5 has demonstrated system-related geometrical distortion was present in all $3 \mathrm{~T}$ and $7 \mathrm{~T} \mathrm{MRI} \mathrm{and} \mathrm{increased} \mathrm{with} \mathrm{an} \mathrm{increasing} \mathrm{distance}$ from the magnetic isocentre. Although geometrical distortion was $\leq 1 \mathrm{~mm}$ within the clinically relevant volume around the isocentre, with the introduction of $7 \mathrm{~T} \mathrm{MRI}$ to the clinic geometrical accurate MR protocols optimized for radiation treatment planning should be further developed.

\section{Autophagy inhibition}

$\mathrm{CQ}$ is a 4-aminoquinoline, commonly used as treatment and prophylaxis for infection with plasmodium (malaria). It has been used for decades for this purpose, but has also been prescribed chronically for indications such as rheumatoid arthritis, lupus erythematosus and other connective tissue and skin disorders. In addition, it has recently been rediscovered as a possible anti-viral against the outbreak of the new coronavirus (COVID-19) $)^{56}$ CQ is relatively non-toxic, with daily doses up to $600 \mathrm{mg}$ per day in patients with lupus ${ }^{57}$. Chapter 6 explores the safety, pharmacokinetics and maximum tolerated dose of $C Q$ in combination with radiotherapy and daily $T M Z$ in patients with a newly diagnosed GBM. The underlying hypothesis of this study is that at times of cellular stress, autophagy is induced within the cell. This includes the formation of double-membraned vesicles known as autophagosomes that engulfs cellular proteins and organelles for delivery to the lysosome after which the contents are degraded. These contents can be recycled or used to fuel metabolic pathways necessary for cell survival ${ }^{58}$. CQ has weak base properties, which allows accumulation in the lysosomes and a rise in intralysosomal $\mathrm{pH}$. This prevents autophagosome lysosome fusion and thereby inhibits autophagy making malignant cells more vulnerable for cellular stress such as radiation treatment or chemotherapy ${ }^{59-61}$. A rise in the autophagy marker LC3b, which is normally degraded during autophagy, confirms autophagy inhibition with $\mathrm{CQ}^{59}$. In the presented phase I trial patients 
received oral CQ daily starting one week before the course of chemoradiation until the end of radiotherapy. Thirteen patients were enrolled in a $3+3$ design between August 2016 and December 2018 at three different dose levels: 200 mg, 300 mg and $400 \mathrm{mg}$. A total of 44 adverse events, possibly related to CQ were registered including electrocardiogram QTc prolongation, irreversible blurred vision and nausea/vomiting resulting in cessation of TMZ or delay of adjuvant cycles. Limitations of this study are inherent to the $3+3$ study design and include a low likelihood of selecting the true maximum tolerated dose due to pre-defined dose levels, low patient numbers resulting in a small likelihood of detecting all dose limiting toxicities and the study was not designed to re-escalate the dose if possible. Another major limitation is that direct autophagy inhibition could not be established, which is highly desirable to support the main hypothesis. The study established $200 \mathrm{mg}$ CQ daily in combination with radiotherapy and TMZ as the recommended phase II dose and demonstrated that despite $C Q$ has a well-known toxicity profile it can still elicit significant side effects in combination with radiotherapy and TMZ. Although not powered for a formal statistical analysis, the study confirmed EGFRvIll as a potential predictive biomarker.

Several studies have investigated other mechanisms by which CQ may exert its anti-tumor effects. One study demonstrated that CQ-induced cell death may not be related to autophagy inhibition itself, but to inhibition of cholesterol biosynthesis by autophagy related pathways ${ }^{62}$. Moreover, there are several investigators that suggest an important role in cell metabolism including the amino acid ${ }^{63}$, glucose ${ }^{64}$ and mitochondrial metabolism ${ }^{65}$. Another group has recently published a study protocol for a phase IB/II clinical trial in which they investigate both metformin and $\mathrm{CQ}$ in patients with IDH1-mutated or IDH2-mutated solid tumors ${ }^{66}$. They hypothesize that in addition to autophagy inhibition, CQ can also inhibit the conversion of glutamate to aKG by glutamate dehydrogenase. Isocitrate dehydrogenase (IDH) 1/2-mutated glioma may be especially responsive as these tumor are dependent on aKG for their metabolism ${ }^{63}$. Furthermore, there is evidence that CQ can normalize tumor vessel structure and function, thereby increasing perfusion ${ }^{67}$. This effect was dose-dependent and may lead to improved chemotherapy delivery. There are also indications that autophagy plays an important role in the tumor-specific immune response. This raises some concerns as one study demonstrated that autophagy inhibition with CQ may suppress this ATP release and thereby inhibit the tumorspecific immune response ${ }^{68,69}$. Conversely, other authors have shown that treatment of cells with CQ results in a significant cellular accumulation Toll-like-receptor 4 on the cell surface which binds to dendritic cells and macrophages thereby increasing the tumor specific immune response. ${ }^{70}$

With the emergence of new potentially beneficial therapies such as $C Q$, clinical trials suggest that a subgroup of patients may benefit from specific treatments. Identification and validation of predictive biomarkers could allow for implementation of targeted therapy based on molecular features of specific tumors ${ }^{71}$. However, GBM do not only display their heterogeneity both inter- and intra-tumoral but also in time, 
with a different set of molecular alterations at recurrence ${ }^{72-75}$. This poses a significant challenge for targeted therapies as tissue analysis of the entire tumor is often limited due to limitations in surgical debulking both in the primary setting or at recurrence. Moreover, heterogeneity at single cell level may exist i.e. multiple genomic alterations within redundant pathways or multiple unique variants of a single gene, resulting in heterogeneity in response at cell level ${ }^{72,73,76}$. Glioma Stem Cells (GSC), which can sustain self-renewal, cause persistent proliferation and have the ability to facilitate tumor initiation, may be one of the main causes of tumor heterogeneity ${ }^{77}$. As GSC are a major cause of treatment resistance against radiotherapy and chemotherapy, specifically targeting may significantly improve treatment response. One of the potential genes found in GSC which has also been identified as a potential predictive marker in this thesis is EGFRvIII ${ }^{8}$. EGFR is a transmembrane receptor kinase of the ERBB family which activation regulates multiple downstream physiological and pathological processes. Amplification and gene rearrangement of EGFR (i.e. deletion in exons $2-7$ ) is frequently observed in GBM and results in the expression of variant EGFRvIII ${ }^{9}$. EGFRvIII does not contain a ligand-binding domain and is constitutively active, which results in cell proliferation, angiogenesis, invasion and provides cells with a survival advantage through autophagy ${ }^{80-82}$. It has been observed in approximately $20-40 \%$ of $\mathrm{GBM}^{72,83,84}$. As EGFRvIll is only expressed by malignant cells and not healthy tissue, it is a very attractive target for therapy. Previous pre-clinical work by MAASTRO lab has shown that EGFRvIll is a potential predictive marker for CQ in newly diagnosed GBM ${ }^{59,82,85}$. Although the phase I study presented in Chapter 6 is small, results also showed indications of EGFRvIII as a potential predictive biomarker. Several other anti-EGFR/EGFRvIII therapies are currently under investigation. These include small molecule tyrosine kinase inhibitors, antibodies, vaccines, chimeric antigen receptor (CAR) T-cells and RNA-based therapies ${ }^{86}$. In patients with non-small cell lung cancer EGFR inhibitors Gefitinib and Erlotinib have demonstrated clinical efficacy in patients with an EGFR mutation. However in GBM, studies have not been able to show a clinically significant survival benefit so far ${ }^{87-90}$. Treatment with antiEGFR antibodies such as cetuximab has also shown limited activity ${ }^{91,92}$. And although Rindopepimut, a vaccine targeting EGFRvIll, was promising in early clinical trials, it did not increase survival in combination with TMZ in newly diagnosed EGFRvIll + $\mathrm{GBM}^{93}$. This treatment resistance is seemingly caused by intratumoral heterogeneity, compensatory signalling pathways and secondary mutations. This highlights the need for a better understanding of the biological pathways in GBM and potential combination therapies. It is therefore conceivable that in the future $C Q$ may be combined with other targeted therapies in the treatment of GBM. 
Chapter 8 - Future Perspectives 


\section{Decision support systems}

As indicated in Chapters 2 and 3 of this thesis there is still a lot of work to be done before decision support systems for GBM can be implemented in clinical practice. However, it is expected that within five years these systems will be able to present treating physicians with an accurate estimation of tumor control, toxicity, quality of life and cost effectiveness of a treatment. Once these systems have been integrated into daily clinical practice, there is one more important step to be taken: provide patients with the same information as their treating physicians receive. As patients often value outcomes very differently than their treating physician(s), wellinformed shared decision making is an essential part of personalized medicine ${ }^{94}$. By empowering the patient to actively participate in the decision-making higher levels of satisfaction with their treatment will be achieved, eventually resulting in a better short and long-term QoL. Although a treating physician presents all the relevant risks and benefits of a treatment during a consultation interview, this may not take into account the patient's personal values and risk-balancing preferences between treatment benefits and harms such as quality-of-life versus quantity-of-life. Eliciting patient's values and preferences is a complex task, as values of another person are difficult to anticipate and can be influenced by clinician's views ${ }^{95}$. This would involve a new way of medical counselling, as physicians have difficulty predicting the treatment decisional preferences of their patients. Furthermore, presentation of unbiased medical information should be tailored to the patient's needs, for instance via interactive online decision aids and videos. These are currently not yet available for glioma patients, and should be developed in the coming years to improve the patient's ability to make the best decision for their individual situation. Furthermore, indicators of decision quality measuring patient's expectations, satisfaction, and regret should be investigated. However, this does present an additional challenge. Accounting for patient's preferences will affect the way in which randomized trials can be conducted, especially when the study is designed to compare a "one size fits all" treatment to an individualized treatment. In a classical trial design, where treatment arms with different "one size fits all" treatments are compared, the primary endpoint (OS, local control, etc.) is defined unequivocally. This is cannot be the case in trials with a treatment arm involving individualized treatment, as certain patients will for instance attach more importance to QoL related aspects than to tumor control. This explicitly and irrevocably inserts the patient's selection bias into the treatment selections process. The challenge will be to still build a reasonable level of evidence. This is clearly a field where more research is necessary.

The radiomics study presented in Chapter 3 has several limitations such as a small number of patients, manual delineation of the tumor on MR images registered to CT images with inter- and intraobserver variability and a lack of standardization in imaging protocols between the three participating institutes. Some of these may be 
solved by the introduction of deep learning algorithms to characterize gliomas ${ }^{96,97}$. Deep learning is a subset of machine learning inspired by biological neural networks in which a deep neural network architecture cascades through multiple nodes in multiple layers, where nodes and layers use the output of the previous nodes and layers as input ${ }^{98}$. Two major differences with radiomics are that deep learning does not require a (manual) delineation of the tumor or human-designed feature extraction and that it is capable of determining more abstract and higher-order relationships between features and data ${ }^{99}$. Results in glioma have already been very promising and an exceptional growth in published studies is expected ${ }^{96,100 .}$

In summary, in addition to expansion of therapeutic options, decision support systems for GBM require further development. This includes the implementation of (advanced) prognostic and predictive techniques such deep learning into the NIGT platform, but in parallel the patient's perspective should also be incorporated in clinical trials. The development of easily accessible decision aids will result in truly personalized medicine for GBM and is expected within 5 years.

\section{Ultra-high field MRI}

So far 7 T MRI has shown to be especially valuable for cerebrovascular imaging, leading to the hypothesis that using $7 \mathrm{~T} \mathrm{MRI} \mathrm{to} \mathrm{visualize} \mathrm{microvascularisation} \mathrm{outside}$ the contrast-enhanced volume on MRI would permit an improved delineation of highly vascularized GBM for RTP $33-36,101$. In Chapters 4 and 5 we have shown that the implementation of $7 \mathrm{~T} \mathrm{MRI} \mathrm{within} \mathrm{existing} \mathrm{radiotherapy} \mathrm{practice} \mathrm{is} \mathrm{feasible.} \mathrm{This} \mathrm{can}$ be done in as short a period of a one year. Unfortunately, the potential benefit of UHF MRI for RTP in GBM and most importantly the effect on clinical outcome still needs to be demonstrated. So far only one group has investigated 7 T MRI for RTP ${ }^{39}$. They concluded that 7 T FLAIR MRI could enhance visualization of tumor infiltration as well as neighbouring white matter tracts and OAR and thus could potentially alter treatment planning volumes. Further research is required to confirm these findings before clinical implementation. In parallel both researchers and manufacturers should work on enhancing image quality of $7 \mathrm{TMRI}$ as some issues, such as susceptibility artefacts in the frontobasal region and in the temporal lobe, still remain to be solved.

Continuous technological advances within modern day radiotherapy ensure accurate treatment of the tumor while protecting OAR. These include developments such as volumetric modulated arc therapy, cone-beam computed tomography and proton therapy. Recently proton therapy has become available at three centres in the Netherlands: Groningen, Delft and Maastricht. It can be anticipated that novel imaging modalities including UHF-MRI, as described in Chapters 4 and 5, with an increased SNR and enhanced contrast will provide an increased ability to detect and quantify tumour activity and anatomical boundaries and thereby improve the delineation of both tumor and $O A R^{40,101,102}$. Proton therapy may greatly benefit from these developments as proton therapy allows an increased sparing of the 
OAR compared to photons due to the finite range of the protons ${ }^{102}$. In proton radiotherapy the particle beam has relatively low entry dose and can deposit its energy over a narrow range (the Bragg peak) ${ }^{103}$. Treatment with protons allows for very low radiation dose to be deposited in healthy tissue distally from the Bragg peak, making it especially attractive for neuro-oncological purposes where critical OAR are often situated immediately adjacent to the target volume. This aspect of proton therapy can be maximally utilized if both target and OAR are delineated bases on a high quality images and a high contrast-to-noise ratio as is the case with UHFMRI. However, the range of protons is very sensitive to anatomical changes (e.g. due to tumor shrinkage) and tissue heterogeneities along the beam path (e.g. air-filled cavities as the mastoid), which could result in a displacement of the Bragg peak into an OAR. This can potentially be avoided by selecting beam angles based on the delineated organs. In summary, UHF-MRI can help both utilize the full potential of proton therapy and avoid some of its potential pitfalls, making further research towards implementing UHF-MRI for proton therapy, especially for neuro-oncological purposes, very desirable.

Another recent development in image-guided radiotherapy is the clinical introduction of the MR-linear accelerator (MRL), which is a hybrid device combining a linear accelerator with on-board MR imaging. MRL provides real-time MR imaging to characterize and track anatomical motion ${ }^{104,105}$. In clinical practice the MRL can be used to replace the conebeam-CT for position verification, applied for online adaptive re-planning and treatment response assessment ${ }^{106,107}$. Current potential clinical indications include brain, breast, cervix, oesophagus, lung, oropharynx, pancreas, prostate, and rectum ${ }^{108}$. In glioma and specifically GBM post-operative shifts in anatomy and changes in gross tumor volume have been observed 109. In these cases, an adapted plan on the MRL could potentially lead to improved target coverage with less dose to the OAR. However, the value of MRL for glioma still needs to be determined in clinical trials as the main benefits of MRL lie in adaptation to the anatomy of the day and anatomical changes during treatment delivery which is not expected to occur frequently within the brain. However, for other neurooncological tumors known to have dynamic cysts such as craniopharyngeoma the advantage may be considerable ${ }^{110}$. These patients currently receive weekly MRIs during treatment to identify changes in the cysts after which the treatment plan is adapted accordingly. These adapted treatment plans are however always lagging behind, whereas an online adapted plan based on the MRL would provide an optimal treatment. Moreover, MR-guided stereotactic radiosurgery for intracranial metastases holds promise ${ }^{111}$.

The MRLs currently available make use of low $(0.35 \mathrm{~T})$ and high (1.5 $\mathrm{T}$ ) magnetic field strengths providing lower signal-to-noise ratios than for UHF-MRI at 7 T, but with the benefit of less susceptibility artefacts and geometrical distortion. Quality assurance to minimize the presence of imaging artefacts, obtain high geometrical accuracy and 
implement new clinical RT workflows is essential, making the research presented in Chapters 4 and 5 also in this field of work very relevant.

In summary, an accurate delineation of both the tumor and OAR is essential for treatments with a very sharp penumbra such as stereotactic radiotherapy or a steep dose profile due to the Bragg peak such as proton therapy. It can be expected that with the clinical introduction of $7 \mathrm{~T} \mathrm{MRI,} \mathrm{proton} \mathrm{therapy} \mathrm{treatment} \mathrm{planning} \mathrm{for} \mathrm{brain}$ tumors will be UHF-guided with a more accurate definition of the target volume and OAR. The advantage of MRLs, in particular for online plan adaptation for brain tumors is less obvious and will need to be validated in clinical trials in the coming years. Whether MRLs with high magnetic field strengths provide a clinical advantage over low field systems remains to be determined. It can be expected that within the coming 10 years the major advantages of both proton therapy and MRL will be incorporated into MR-guided proton therapy ${ }^{112}$.

\section{Autophagy inhibition}

In Chapter 6 the recommended phase II dose of CQ in combination with radiotherapy and $\mathrm{TMZ}$ was established. In order to conclusively demonstrate the effect on outcome of CQ phase II/III trials needs to be conducted. For this purpose, a phase II study proposal has already been written. In this study patients with a pathologically confirmed de novo GBM, eligible for concurrent chemoradiation will be randomized between concurrent radio-chemotherapy with daily TMZ alone or concurrent radiotherapy in combination with $200 \mathrm{mg}$ CQ daily. The primary objective of this study is to demonstrate that concurrent radiotherapy with daily TMZ and $\mathrm{CQ}$ improves OS as compared to concurrent chemoradiation alone. The secondary objectives include assessment of quality of life, establishing the safety profile of $C Q$, identification of predictive factors and establishing the effect of CQ alone on tumor hypoxia in vivo. In order to demonstrate EGFRVIII as a predictive biomarker, patients will be stratified according to EGFRvIll status. Based on previous publications 2-year OS was set at $27 \%$ for the control group and $37 \%$ for the experimental treatment group 113,114 . With $5 \%$ level of significance $(\alpha=0.05)$ and $80 \%$ power $(1-\beta=0.80)$, a total of 266 patients would need to be included in this study.

The inclusion of 266 patients with a GBM is one of the main obstacles to perform such a trial in the Netherlands. Annually approximately 700 Dutch patients are diagnosed with a GBM of which 500 are potentially eligible for inclusion. If every centre in the Netherlands would participate, with an expected $10 \%$ inclusion rate, it would still take 5 years to complete the study. Therefore, an internationally set-up trial in cooperation with a major research platform such as e.g. the European Organisation for Research and Treatment of Cancer (EORTC) is an absolute necessity. The other main obstacle is acquiring funding for such a large study. Investigator-initiated trials that are not sponsored by large pharmaceutical companies often find it hard to acquire enough funding to come from basic discovery to clinical application. The Bright Focus 
Foundation has described this phenomenon as an "uphill battle" in which every step is more difficult than the last ${ }^{115}$. CQ has already taken the second step between translational research and early clinical trials known as the "funding valley of death" by a generous donation from one of the Dutch brain tumor patient organizations "Stichting Stophersentumoren". However, funding to climb up to phase II and III up the hill has not yet been secured and needs to be established before any further steps can be taken.

To summarize, although many necessary steps have been taken to conduct a large randomized phase 2 trial only a large international collaboration will be able to achieve enough patients and funding to conclusively demonstrate the effect of $C Q$ on outcome in GBM.

To conclude, despite promising innovations in the diagnosis and treatment of GBM the prognosis of patients remains poor. However, with further optimization of decision support systems, imaging modalities, systemic treatments and radiotherapy techniques, a prolonged survival with increased quality of life may be within reach for patients with a GBM. 
1. Aerts HJ, Velazquez ER, Leijenaar RT, et al. Decoding tumour phenotype by noninvasive imaging using a quantitative radiomics approach. Nature communications. 2014;5:4006.

2. Lambin $\mathrm{P}$, Rios-Velazquez $\mathrm{E}$, Leijenaar $\mathrm{R}$, et al. Radiomics: extracting more information from medical images using advanced feature analysis. Eur $\mathrm{J}$ Cancer. 2012;48(4):441-446.

3. Lambin P, Leijenaar RTH, Deist TM, et al. Radiomics: the bridge between medical imaging and personalized medicine. Nat Rev Clin, Oncol. 2017 Dec(1759-4782 ).

4. Collins GS, Reitsma JB, Altman DG, Moons KG. Transparent reporting of a multivariable prediction model for individual prognosis or diagnosis (TRIPOD): the TRIPOD statement. Br, J Cancer. 2015;Jan 20(1532-1827).

5. Lambin P, Leijenaar RTH, Deist TM, et al. Radiomics: the bridge between medical imaging and personalized medicine. Nature reviews Clinical oncology. 2017;14(12):749-762.

6. Kickingereder P, Gotz M, Muschelli J, et al. Large-scale Radiomic Profiling of Recurrent Glioblastoma Identifies an Imaging Predictor for Stratifying AntiAngiogenic Treatment Response. Clin Cancer Res. 2016;22(23):5765-5771.

7. Itakura $\mathrm{H}, \mathrm{Achrol} A \mathrm{~S}$, Mitchell $L A$, et al. Magnetic resonance image features identify glioblastoma phenotypic subtypes with distinct molecular pathway activities. Sci Transl, Med. 2015;7(303):303ra138.

8. Gevaert O, Mitchell LA, Achrol AS, et al. Glioblastoma multiforme: exploratory radiogenomic analysis by using quantitative image features. Radiology. 2014;273(1):168-174.

9. Grossmann P, Gutman DA, Dunn WD, Jr., Holder CA, Aerts HJ. Imaging-genomics reveals driving pathways of MRI derived volumetric tumor phenotype features in Glioblastoma. BMC cancer. 2016;16:611.

10. Yang D, Rao G, Martinez J, Veeraraghavan A, Rao A. Evaluation of tumor-derived MRI-texture features for discrimination of molecular subtypes and prediction of 12-month survival status in glioblastoma. Med Phys. 2015;42(11):6725-6735.

11. Cui Y, Tha KK, Terasaka S, et al. Prognostic Imaging Biomarkers in Glioblastoma: Development and Independent Validation on the Basis of Multiregion and Quantitative Analysis of MR Images. (1527-1315 (Electronic)).

12. Weller $M$, van den Bent $M$, Tonn JC, et al. European Association for NeuroOncology (EANO) guideline on the diagnosis and treatment of adult astrocytic and oligodendroglial gliomas. Lancet, Oncol. 2017;18(6):e315-e329.

13. Loizou CP, Petroudi S, Seimenis I, Pantziaris M, Pattichis CS. Quantitative texture analysis of brain white matter lesions derived from T2-weighted MR images in MS patients with clinically isolated syndrome. Journal of neuroradiology Journal de neuroradiologie. 2015;42(2):99-114.

14. Madabhushi A, Udupa JK. New methods of MR image intensity standardization via generalized scale. Medical physics. 2006;33(9):3426-3434. 
15. Institute NC. Dictionary of cancer terms. http://www.cancer.gov/ dictionary?CdrD=56717. Accessed 06-05, 2020.

16. Walsh S, de Jong EEC, van Timmeren JE, et al. Decision Support Systems in Oncology. JCO Clin Cancer Inform. 2019;3:1-9.

17. Weller M, Stupp R Fau - Hegi ME, Hegi Me Fau - van den Bent M, et al. Personalized care in neuro-oncology coming of age: why we need MGMT and $1 p / 19 q$ testing for malignant glioma patients in clinical practice. Neuro-oncology. 2012;14(Suppl 4:iv):100-108.

18. Louis DN, Perry A, Reifenberger G, et al. The 2016 World Health Organization Classification of Tumors of the Central Nervous System: a summary. Acta neuropathologica. 2016;131(6):803-820.

19. Wick W, Roth $\mathrm{P}$, Hartmann C, et al. Long-term analysis of the NOA-04 randomized phase III trial of sequential radiochemotherapy of anaplastic glioma with PCV or temozolomide. Neuro-oncology. 2016;18(11):1529-1537.

20. van den Bent MJ, Dubbink Hj Fau - Sanson M, Sanson M Fau - van der LeeHaarloo CR, et al. MGMT promoter methylation is prognostic but not predictive for outcome to adjuvant PCV chemotherapy in anaplastic oligodendroglial tumors: a report from EORTC Brain Tumor Group Study 26951. J Clin Oncol. 2009;27(35):5881-5886.

21. Hegi ME, Diserens Ac Fau - Gorlia T, Gorlia T Fau - Hamou M-F, et al. MGMT gene silencing and benefit from temozolomide in glioblastoma. N Engl J Med. 2005;352(10):997-1003.

22. Gilbert MR, Wang M Fau - Aldape KD, Aldape Kd Fau - Stupp R, et al. Dose-dense temozolomide for newly diagnosed glioblastoma: a randomized phase III clinical trial. J Clin Oncol. 2013;31(32):4085-4091.

23. Reifenberger G, Hentschel B Fau - Felsberg J, Felsberg J Fau - Schackert G, et al. Predictive impact of MGMT promoter methylation in glioblastoma of the elderly. Int J Cancer. 2012;131(6):1342-1350.

24. Cairncross G Fau - Berkey B, Berkey B Fau - Shaw E, Shaw E Fau - Jenkins R, et al. Phase III trial of chemotherapy plus radiotherapy compared with radiotherapy alone for pure and mixed anaplastic oligodendroglioma: Intergroup Radiation Therapy Oncology Group Trial 9402. J Clin Oncol. 2006;24(18):2707-2714.

25. Erdem-Eraslan L, Gravendeel La Fau - de Rooi J, de Rooi J Fau - Eilers PHC, et al. Intrinsic molecular subtypes of glioma are prognostic and predict benefit from adjuvant procarbazine, lomustine, and vincristine chemotherapy in combination with other prognostic factors in anaplastic oligodendroglial brain tumors: a report from EORTC study 26951. J Clin Oncol. 2013;31(3):328-336.

26. Weller M, Felsberg J Fau - Hartmann C, Hartmann C Fau - Berger H, et al. Molecular predictors of progression-free and overall survival in patients with newly diagnosed glioblastoma: a prospective translational study of the German Glioma Network. J Clin Oncol. 2009;27(34):5743-5750. 
27. Hartmann C, Hentschel B Fau - Wick W, Wick W Fau - Capper D, et al. Patients with IDH1 wild type anaplastic astrocytomas exhibit worse prognosis than IDH1mutated glioblastomas, and IDH1 mutation status accounts for the unfavorable prognostic effect of higher age: implications for classification of gliomas. Acta neuropathologica. 2010;120(6):707-718.

28. Wick W, Meisner C Fau - Hentschel B, Hentschel B Fau - Platten M, et al. Prognostic or predictive value of MGMT promoter methylation in gliomas depends on IDH1 mutation. Neurology. 2013;81(17):1515-1522.

29. Lustberg $T$, van Soest J, Jochems A, et al. Big Data in radiation therapy: challenges and opportunities. Br J Radiol. 2017;90(1069):20160689.

30. Zerka F, Barakat S, Walsh S, et al. Systematic Review of Privacy-Preserving Distributed Machine Learning From Federated Databases in Health Care. JCO Clin Cancer Inform. 2020;4(184-200).

31. Ladd ME. High-field-strength magnetic resonance: potential and limits. Top Magn Reson Imaging. 2007;18(2):139-152.

32. Zwanenburg JJ, Hendrikse J Fau - Visser F, Visser F Fau - Takahara T, Takahara T Fau - Luijten PR, Luijten PR. Fluid attenuated inversion recovery (FLAIR) MRI at 7.0 Tesla: comparison with 1.5 and 3.0 Tesla. Eur Radiol. 2010;20(4):915-922.

33. Moenninghoff $C$, Maderwald $S$, Theysohn JM, et al. Imaging of adult astrocytic brain tumours with 7 T MRI: preliminary results. Eur Radiol. 2010;20(3):704-713.

34. Paek SL, Chung Ys Fau - Paek SH, Paek Sh Fau - Hwang JH, et al. Early experience of pre-and post-contrast 7.0T MRI in brain tumors. JKorean Med Sci. 2013;28(9):13621372.

35. Christoforidis GA, Yang M, Abduljalil A, et al. "Tumoral pseudoblush" identified within gliomas at high-spatial-resolution ultrahigh-field-strength gradient-echo MR imaging corresponds to microvascularity at stereotactic biopsy. Radiology. 2012;264(1):210-217.

36. Grabner G, Kiesel B, Wohrer A, et al. Local image variance of 7 Tesla SWI is a new technique for preoperative characterization of diffusely infiltrating gliomas: correlation with tumour grade and IDH1 mutational status. Eur Radiol. 2017;27(4):1556-1567.

37. Lupo JM, Li Y, Hess CP, Nelson SJ. Advances in ultra-high field MRI for the clinical management of patients with brain tumors. Curr Opin Neurol. 2011;24(6):605615.

38. Baker GJ, Yadav VN, Motsch S, et al. Mechanisms of glioma formation: iterative perivascular glioma growth and invasion leads to tumor progression, VEGFindependent vascularization, and resistance to antiangiogenic therapy. Neoplasia (New York, NY). 2014;16(7):543-561.

39. Regnery S, Knowles BR, Paech D, et al. High-resolution FLAIR MRI at 7 Tesla for treatment planning in glioblastoma patients. Radiother Oncol. 2019;130:180-184.

40. Eekers DB, In't Ven L, Roelofs E, et al. The EPTN consensus-based atlas for CT- and MR-based contouring in neuro-oncology. Radiother Oncol. 2018;128(1):37-43. 
41. de Rotte AA, van der Kolk Ag Fau - Rutgers D, Rutgers D Fau - Zelissen PMJ, et al. Feasibility of high-resolution pituitary MRI at 7.0 tesla. Eur Radiol. 2010;24(8):20052011.

42. de Rotte AA, Groenewegen A, Rutgers DR, et al. High resolution pituitary gland MRI at 7.0 tesla: a clinical evaluation in Cushing's disease. Eur Radiol. 2016;26(1):271277.

43. Song SW, Son YD, Cho ZH, Paek SH. Experience with 7.0 T MRI in Patients with Supratentorial Meningiomas. J Korean Neurosurg Soc. 2016;59(4):405-409.

44. Barrett TF, Dyvorne HA, Padormo F, et al. First Application of 7-T Magnetic Resonance Imaging in Endoscopic Endonasal Surgery of Skull Base Tumors. World Neurosurg. 2017;103:600-610.

45. Seibert TM, White NS, Kim GY, et al. Distortion inherent to magnetic resonance imaging can lead to geometric miss in radiosurgery planning. Practical radiation oncology. 2016;6(6):e319-e328.

46. Dietrich O, Reiser Mf Fau - Schoenberg SO, Schoenberg SO. Artifacts in 3-T MRI: physical background and reduction strategies. Eur J Radiol. 2008;65(1):29-35.

47. Weygand J, Fuller CD, Ibbott GS, et al. Spatial Precision in Magnetic Resonance Imaging-Guided Radiation Therapy:The Role of Geometric Distortion. Int J Radiat Oncol Biol Phys. 2016;95(4):1304-1316.

48. Bernstein MA KK, Zhou XJ. Handbook of MRI pulse sequences. Cambridge, MA: Elsevier Academic Press; 2004.

49. Walker A, Liney G Fau - Metcalfe P, Metcalfe P Fau - Holloway L, Holloway L. MRI distortion: considerations for MRI based radiotherapy treatment planning. Australasian physical \& engineering sciences in medicine / supported by the Australasian College of Physical Scientists in Medicine and the Australasian Association of Physical Sciences in Medicine. 2014;37(1):103-113.

50. Schmidt MA, Wells EJ, Davison K, Riddell AM, Welsh L, Saran F. Stereotactic radiosurgery planning of vestibular schwannomas: Is MRI at 3 Tesla geometrically accurate? Med Phys. 2017;44(2):375-381.

51. Stanescu T, Jans HS, Wachowicz K, Fallone BG. Investigation of a 3D system distortion correction method for MRimages.J ApplClin Med Phys. 2010;11(1):2961.

52. Baldwin LN, Wachowicz K, Thomas SD, Rivest R, Fallone BG. Characterization, prediction, and correction of geometric distortion in MR images. Medical Physics. 2007;34(2):388-399.

53. Doran SJ, Charles-Edwards L, Reinsberg SA, Leach MO. A complete distortion correction for MR images: I. Gradient warp correction. Physics in medicine and biology. 2005;50(7):1343-1361.

54. Dammann $\mathrm{P}$, Kraff $\mathrm{O}$, Wrede $\mathrm{KH}$, et al. Evaluation of hardware-related geometrical distortion in structural MRI at 7 Tesla for image-guided applications in neurosurgery. Academic radiology. 2011;18(7):910-916. 
55. Damyanovich AZ, Rieker M, Zhang B, Bissonnette JP, Jaffray DA. Design and implementation of a 3D-MR/CT geometric image distortion phantom/ analysis system for stereotactic radiosurgery. Physics in medicine and biology. 2018;63(7):075010.

56. Keyaerts E, Li S Fau - Vijgen L, Vijgen L Fau - Rysman E, et al. Antiviral activity of chloroquine against human coronavirus OC43 infection in newborn mice. Antimicrob Agents Chemother. 2009;53(8):3416-3421.

57. Mavrikakis I, Sfikakis PP, Mavrikakis E, et al. The incidence of irreversible retinal toxicity in patients treated with hydroxychloroquine: a reappraisal. Ophthalmology. 2003;110(7):1321-1326.

58. Mizushima NA-Ohoo. A brief history of autophagy from cell biology to physiology and disease. Nat Cell Biol. 2018;20(5):521-527.

59. Rouschop KM, van den Beucken $T$, Dubois $L$, et al. The unfolded protein response protects human tumor cells during hypoxia through regulation of the autophagy genes MAP1LC3B and ATG5. J Clin Invest. 2010;120(1):127-141.

60. Savarino A, Boelaert JR, Cassone A, Majori G, Cauda R. Effects of chloroquine on viral infections: an old drug against today's diseases? Lancet Infect Dis. 2003;3(11):722-727.

61. Rouschop KM, Ramaekers $\mathrm{CH}$, Schaaf $\mathrm{MB}$, et al. Autophagy is required during cycling hypoxia to lower production of reactive oxygen species. Radiother Oncol. 2009;92(3):411-416.

62. King MA, Ganley IG, Flemington V. Inhibition of cholesterol metabolism underlies synergy between mTOR pathway inhibition and chloroquine in bladder cancer cells. Oncogene. 2016;35(34):4518-4528.

63. Choi MM, Kim Ea Fau - Choi SY, Choi Sy Fau - Kim TU, Kim Tu Fau - Cho S-W, Cho Sw Fau - Yang S-J, Yang SJ. Inhibitory properties of nerve-specific human glutamate dehydrogenase isozyme by chloroquine. J Biochem Mol Biol. 2007;40(6):1077-1082.

64. Cotton Dw Fau - Sutorius AH, Sutorius AH. Inhibiting effect of some antimalarial substances on glucose-6-phosphate dehydrogenase. Nature. 1971;233(5316):197.

65. Deepalakshmi PD, Parasakthy K Fau - Shanthi S, Shanthi S Fau - Devaraj NS, Devaraj NS. Effect of chloroquine on rat liver mitochondria. Indian J Exp Biol. 1994;32(11):797-799.

66. Molenaar RJ, Coelen RJS, Khurshed M, et al. Study protocol of a phase IB/II clinical trial of metformin and chloroquine in patients with IDH1-mutated or IDH2mutated solid tumours. BMJ Open. 2017;7(6):e014961.

67. Maes $H$, Kuchnio $A$, Peric $A$, et al. Tumor vessel normalization by chloroquine independent of autophagy. Cancer Cell. 2014;26(2):190-206.

68. Michaud M, Martins I, Sukkurwala $A Q$, et al. Autophagy-dependent anticancer immune responses induced by chemotherapeutic agents in mice. Science (New York, NY). 2011;334(6062):1573-1577. 
69. Ko A, Kanehisa A, Martins I, et al. Autophagy inhibition radiosensitizes in vitro, yet reduces radioresponses in vivo due to deficient immunogenic signalling. Cell death and differentiation. 2014;21(1):92-99.

70. Husebye $\mathrm{H}$, Halaas $\mathrm{O}$, Stenmark $\mathrm{H}$, et al. Endocytic pathways regulate Toll-like receptor 4 signaling and link innate and adaptive immunity. The EMBO journal. 2006;25(4):683-692.

71. Oldenhuis CN, Oosting Sf Fau - Gietema JA, Gietema Ja Fau - de Vries EGE, de Vries EG. Prognostic versus predictive value of biomarkers in oncology. Eur J Cancer. 2008;44(7):946-953.

72. Brennan CW, Verhaak Rg Fau - McKenna A, McKenna A Fau - Campos B, et al. The somatic genomic landscape of glioblastoma. Cell. 2013;155(2):462-477.

73. Patel AP, Tirosh I, Trombetta JJ, et al. Single-cell RNA-seq highlights intratumoral heterogeneity in primary glioblastoma. Science (New York, NY). 2014;344(6190):1396-1401.

74. Johnson $\mathrm{BE}$, Mazor $\mathrm{T}$, Hong $\mathrm{C}$, et al. Mutational analysis reveals the origin and therapy-driven evolution of recurrent glioma. Science (New York, NY). 2014;343(6167):189-193.

75. Draaisma K, Chatzipli A, Taphoorn M, et al. Molecular Evolution of IDH WildType Glioblastomas Treated With Standard of Care Affects Survival and Design of Precision Medicine Trials: A Report From the EORTC 1542 Study. J Clin Oncol. 2020;38(1):81-99.

76. Szerlip NJ, Pedraza A Fau - Chakravarty D, Chakravarty D Fau - Azim M, et al. Intratumoral heterogeneity of receptor tyrosine kinases EGFR and PDGFRA amplification in glioblastoma defines subpopulations with distinct growth factor response. Proc Natl Acad Sci U S A. 2012;109(8):3041-3046.

77. Reya T, Morrison Sj Fau - Clarke MF, Clarke Mf Fau - Weissman IL, Weissman IL. Stem cells, cancer, and cancer stem cells. Nature. 2001;414(6859):105-111.

78. Emlet DR, Gupta P Fau - Holgado-Madruga M, Holgado-Madruga M Fau - Del Vecchio $C A$, et al. Targeting a glioblastoma cancer stem-cell population defined by EGF receptor variant III. Cancer Res. 2014;74(4):1238-1249.

79. Furnari FB, Cloughesy TF, Cavenee WK, Mischel PS. Heterogeneity of epidermal growth factor receptor signalling networks in glioblastoma. Nat Rev Cancer. 2015;15(5):302-310.

80. Feng $\mathrm{H}, \mathrm{Hu} \mathrm{B}$, Vuori $\mathrm{K}$, et al. EGFRvIll stimulates glioma growth and invasion through PKA-dependent serine phosphorylation of Dock180. Oncogene. 2014;33(19):2504-2512.

81. Bonavia R, Inda Mm Fau - Vandenberg S, Vandenberg S Fau - Cheng SY, et al. EGFRvIll promotes glioma angiogenesis and growth through the NF-kappaB, interleukin-8 pathway. Oncogene. 2012;31(36):4054-4066.

82. Jutten $B$, Keulers TG, Schaaf MB, et al. EGFR overexpressing cells and tumors are dependent on autophagy for growth and survival. Radiother Oncol. 2013;108(3):479-483. 
83. Maire CL, Ligon KL. Molecular pathologic diagnosis of epidermal growth factor receptor. Neuro-oncology. 2014;16 Suppl 8:viii1-6.

84. Shinojima N, Tada K, Shiraishi S, et al. Prognostic value of epidermal growth factor receptor in patients with glioblastoma multiforme. Cancer Res. 2003;63(20):69626970.

85. Jutten B, Keulers TG, Peeters HJM, et al. EGFRvIll expression triggers a metabolic dependency and therapeutic vulnerability sensitive to autophagy inhibition. Autophagy. 2018;14(2):283-295.

86. An Z, Aksoy O, Zheng T, Fan QW, Weiss WA. Epidermal growth factor receptor and EGFRvIll in glioblastoma: signaling pathways and targeted therapies. Oncogene. 2018;37(12):1561-1575.

87. Chakravarti A, Wang M Fau - Robins HI, Robins Hi Fau - Lautenschlaeger T, et al. RTOG 0211: a phase 1/2 study of radiation therapy with concurrent gefitinib for newly diagnosed glioblastoma patients. Int J Radiat Oncol Biol Phys. 2013;85(5):1206-1211.

88. Raizer JJ, Abrey Le Fau - Lassman AB, Lassman Ab Fau - Chang SM, et al. A phase II trial of erlotinib in patients with recurrent malignant gliomas and nonprogressive glioblastoma multiforme postradiation therapy. Neuro Oncol. 2010;12(1):95-103.

89. Prados MD, Chang Sm Fau - Butowski N, Butowski N Fau - DeBoer R, et al. Phase II study of erlotinib plus temozolomide during and after radiation therapy in patients with newly diagnosed glioblastoma multiforme or gliosarcoma. J Clin Oncol. 2009;27(4):579-584.

90. Uhm JH, Ballman Kv Fau - Wu W, Wu W Fau - Giannini C, et al. Phase II evaluation of gefitinib in patients with newly diagnosed Grade 4 astrocytoma: Mayo/North Central Cancer Treatment Group Study N0074. Int J Radiat Oncol Biol Phys. 2011;80(2):347-353.

91. Neyns B, Sadones J Fau - Joosens E, Joosens E Fau - Bouttens F, et al. Stratified phase II trial of cetuximab in patients with recurrent high-grade glioma. Ann Oncol. 2009;20(9):1596-1603.

92. Chong DQ, Toh XY, Ho IA, et al. Combined treatment of Nimotuzumab and rapamycin is effective against temozolomide-resistant human gliomas regardless of the EGFR mutation status. BMC Cancer. 2015;11(15):255.

93. Weller M, Butowski N, Tran DD, et al. Rindopepimut with temozolomide for patients with newly diagnosed, EGFRvlll-expressing glioblastoma (ACT IV): a randomised, double-blind, international phase 3 trial. Lancet Oncol. 2017;18(10):1373-1385.

94. Fagerlin A Fau - Pignone M, Pignone M Fau - Abhyankar P, Abhyankar P Fau Col N, et al. Clarifying values: an updated review. BMC Med Inform Decis Mak. 2013;13: Suppl 2:S8.

95. Mulley AG, Trimble C, Elwyn G. Stop the silent misdiagnosis: patients' preferences matter. Bmj. 2012;345:e6572.

96. Li Z, Wang YA-O, Yu J, Guo Y, Cao W. Deep Learning based Radiomics (DLR) and its usage in noninvasive IDH1 prediction for low grade glioma. Sci Rep. 2017;7(1):5467. 
97. LeCun Y, Bengio Y, Hinton G. Deep learning. Nature. 2015;521 (7553):436-444.

98. Zerka F, Barakat S, Walsh S, et al. Systematic Review of Privacy-Preserving Distributed Machine Learning From Federated Databases in Health Care. JCO Clin Cancer Inform. 2020;4:184-200.

99. Shaver MM, Kohanteb PA, Chiou C, et al. Optimizing Neuro-Oncology Imaging: A Review of Deep Learning Approaches for Glioma Imaging. Cancers (Basel). 2019;11(6).

100. Chang PA-O, Grinband JA-O, Weinberg BA-O, et al. Deep-Learning Convolutional Neural Networks Accurately Classify Genetic Mutations in Gliomas. AJNR Am J Neuroradiol. 2018;39(7):1201-1207.

101. Balchandani P, Naidich TP. Ultra-High-Field MR Neuroimaging. AJNR Am J Neuroradiol. 2015;36(7):1204-1215.

102. Baumann $M$, Krause $M$, Overgaard J, et al. Radiation oncology in the era of precision medicine. Nat Rev Cancer. 2016;16(4):234-249.

103. Jakel O. Medical physics aspects of particle therapy. Radiat Prot Dosimetry. 2009;137(1-2):156-166.

104. Corradini SA-Ohoo, Alongi F, Andratschke N, et al. MR-guidance in clinical reality: current treatment challenges and future perspectives. Radiat Oncol. 2019;14(1):92.

105. Lagendijk JJ, Raaymakers BW, van Vulpen M. The magnetic resonance imaginglinac system. Semin Radiat Oncol. 2014;24(3):207-209.

106. van der Heide UA, Houweling Ac Fau - Groenendaal G, Groenendaal G Fau - BeetsTan RGH, Beets-Tan Rg Fau - Lambin P, Lambin P. Functional MRI for radiotherapy dose painting. Magnetic resonance imaging. 2012;30(9):1216-1223.

107. van Rossum PS, van Lier $A L$, van Vulpen $M$, et al. Diffusion-weighted magnetic resonance imaging for the prediction of pathologic response to neoadjuvant chemoradiotherapy in esophageal cancer. Radiother Oncol. 2015;115(2):163-170.

108. Kerkmeijer LG, Fuller CD, Verkooijen HM, et al. The MRI-Linear Accelerator Consortium: Evidence-Based Clinical Introduction of an Innovation in Radiation Oncology Connecting Researchers, Methodology, Data Collection, Quality Assurance, and Technical Development. (2234-943X (Print)).

109. Kim TG, Lim DH. Interfractional variation of radiation target and adaptive radiotherapy for totally resected glioblastoma. Journal of Korean medical science. 2013;28(8):1233-1237.

110. Jensterle $M$, Jazbinsek $S$, Bosnjak R, et al. Advances in the management of craniopharyngioma in children and adults. Radiol Oncol. 2019;53(4):388-396.

111. Wen N, Kim J, Doemer A, et al. Evaluation of a magnetic resonance guided linear accelerator for stereotactic radiosurgery treatment. Radiother Oncol. 2018;127(3):460-466.

112. Hoffmann A, Oborn B, Moteabbed M, et al. MR-guided proton therapy: a review and a preview. Radiation oncology (London, England). 2020;15(1):129. 
113. Stupp R, Hegi ME, Mason WP, et al. Effects of radiotherapy with concomitant and adjuvant temozolomide versus radiotherapy alone on survival in glioblastoma in a randomised phase III study: 5-year analysis of the EORTC-NCIC trial. Lancet Oncol. 2009;10(5):459-466.

114. Sotelo J, Briceno E, Lopez-Gonzalez MA. Adding chloroquine to conventional treatment for glioblastoma multiforme: a randomized, double-blind, placebocontrolled trial. Ann Intern Med. 2006;144(5):337-343.

115. BrightFocus Foundation. An Uphill Battle. 2013 


\section{Summary}

Gliomas are the mostcommon type of primary malignant tumors of the central nervous system in adult patients, with an annual incidence of approximately 1200 patients in the Netherlands. Patients with a glioblastoma (GBM), the most aggressive type of glioma, are presented with a dismal prognosis. Despite aggressive multimodality regimens including surgical resection, radiation therapy and chemotherapy curation cannot be achieved, recurrence is inevitable and median overall survival is only 15 months. The prognosis for patients with a GBM has not improved significantly over the last 15 years, despite extensive research on how to tackle inter-and intratumoral heterogeneity. In this thesis several innovations in the diagnosis and treatment of GBM are investigated.

Part I considers methods to monitor and predict treatment response which can be included into clinical decision support systems, which are developed in order to aid physicians and patients to choose an optimal treatment strategy. Decision support systems can include all available medical data such as patient demographics and comorbidities, tumor characteristics (both phenotypical and molecular) and treatment characteristics. Chapter 2 discusses the already established approaches as well as future diagnostics used for monitoring and prediction of treatment response in patients with a GBM, introducing the "noninvasive glioblastoma testing" (NIGT) platform. The NIGT platform consists of a multimodal diagnostic approach which tries to capture the complexity and heterogeneity of GBM with the use of conventional images techniques such as magnetic resonance imaging (MRI) and nuclear imaging, enhanced by computational approaches, and the development of circulating biomarkers. Upon initial presentation, standard imaging approach of GBM patients consists of both $C T$ and MRI imaging. The advantages of CT scans over MRI imaging are that CT scans are relatively fast, cheap and widely available. In Chapter 3 a CT radiomics signature for survival in GBM patients treated by radiotherapy is described. This model was compared to two models based on important clinical parameters. The clinical model based on age and WHO performance status and radiomics model both demonstrated a comparable discriminative signature, which merits research to establish the additive value of $\mathrm{CT}$ radiomics. Using methods such as radiomics integrated in the NIGT platform, decision support models for GBM can be created both in the primary and the recurrent setting. This can guide clinicians in selecting the appropriate treatment strategy for patients with a GBM, so-called personalized medicine.

Part Il discusses relevant technical aspects of 7 Tesla (T) MRI for GBM and the necessary steps to integrate this into radiation treatment planning. Ultra-high field strength MRI result in a higher contrast-to-noise ratio compared to conventional high-field strength MRI at 1.5T or $3 \mathrm{~T}$ that is widely available in diagnostic radiology. Chapter 4 reports on a preparatory study in which the technical feasibility of incorporating 7T 
MRI into the neurosurgical navigation and radiation treatment planning systems was investigated. The MRI scans were well tolerated by the volunteers. Regional loss of signal and contrast could be minimized by the use of dielectric pads. Image transfer and processing did not degrade image quality. However, susceptibility artefacts were observed in both the cortex and subcortical white matter at close proximity to airtissue interfaces. The system-related spatial uncertainty of geometrical distortioncorrected MP2RAGE pulse sequences was $\leq 2 \mathrm{~mm}$. In conclusion, the integration of high-quality and geometrically-reliable 7T MR images into neurosurgical navigation and radiation treatment planning software is technically feasible and safe.

The geometrical accuracy of 7T MRI was further investigated in Chapter 5. This study aimed to investigate the clinical applicability of anatomical 7T MRI in comparison to 3T MRI and CT for reliable high-precision radiotherapy by evaluating the magnitude and location of hardware-related geometrical distortion. Three methods were applied: 1) global mean absolute deviation, 2) local mean absolute deviation and 3) Euclidean distances directly between the point of interest-coordinates in the image. System related geometrical distortion was present in all 3T and 7T MRI and increased with increasing distance from the magnetic isocentre, even though vendor provided correction methods and 2nd order 3D shimming were applied. Geometrical distortion of $\leq 1 \mathrm{~mm}$ could be assured near the magnetic isocentre, but was larger in peripheral regions. This implies that 7T MRI can be applied for high-precision radiation treatment planning in central brain regions with clinically acceptable levels of spatial uncertainty, under the assumption that tissue-related geometrical distortion can be ignored. For peripheral regions, applying 7T MR protocols for radiation treatment planning should incorporate geometrical distortion in tumour-site specific planning target volume margins for treatment uncertainties.

In Part III treatment aspects, specifically the safety of autophagy inhibition through the addition of chloroquine to chemoradiation for GBM in a phase lb trial, are discussed. The lysomotropic drug, chloroquine, inhibits autophagy by blocking its final degradative step. This results in a reduction of hypoxic regions within a tumor, making the cells more susceptible to radiation treatment. Chapter 6 presents the results of a phase I trial in which chloroquine concurrent to radiotherapy and temozolomide is investigated in patients with a newly diagnosed GBM. Thirteen patients were included in the study. A total of 44 reversible adverse events, possibly related to chloroquine, were registered; including electrocardiogram QTc prolongation, irreversible blurred vision and nausea/vomiting. Median overall survival was 16 months, with the longest survival rates seen in EGFRvIII + mutated GBM. A daily dose of $200 \mathrm{mg}$ chloroquine was determined as the maximum tolerated dose when combined with radiotherapy and concurrent temozolomide for newly diagnosed GBM. EGFRvIll was identified as a potential predictive biomarker. Favorable toxicity and promising survival in EGFRvIll + patients supports further clinical studies in GBM. 


\section{Samenvatting}

Gliomenzijn demeestvoorkomendeprimairehersentumoren bijvolwassenen meteen jaarlijkse incidentie van 1200 patiënten in Nederland. Patiënten met een glioblastoom (GBM), de meest agressieve vorm van hersentumoren, hebben een slechte prognose. Ondanks een uitgebreide behandeling bestaande uit een resectie, radiotherapie en chemotherapie hebben zij een mediane overleving van slechts 15 maanden. Sinds 2005 is de prognose van deze patiënten, ondanks uitgebreid onderzoek, nauwelijks verbeterd. In dit proefschrift worden verschillende diagnostische en therapeutische innovaties onderzocht. Het proefschrift is opgebouwd uit 3 delen.

In deel I worden verschillende methoden beschreven waarmee de effecten van een behandeling op de overleving kunnen worden voorspeld, en de behandeling geëvalueerd kan worden. Deze informatie kan worden opgenomen in beslismodellen, waardoor in de toekomst de optimale behandeling voor de individuele patiënt kan worden gekozen. In hoofdstuk 2 worden deze methoden uitgebreid beschreven en wordthet"noninvasive glioblastoma testing"(NIGT) platform gepresenteerd. Het NIGTplatform is een multimodale benadering waarin de heterogeniteit van het GBM als geheel kan worden beoordeeld middels conventionele beeldvorming zoals magnetic resonance imaging (MRI) en positronemissietomografie-scans, geavanceerde computermodellen en bloed biomarkers. Ten tijde van de diagnose krijgen de meeste patiënten met een verdenking op een hersentumor een Computed Tomography (CT) -scan. CT-scans zijn relatief snel, goedkoop en wereldwijd beschikbaar. Daarom wordt in hoofdstuk 3 een $\mathrm{CT}$ radiomics model beschreven, waarmee een voorspelling kan worden gedaan van de overleving van patiënten met een GBM na radiotherapie. Dit model wordt vergeleken met 2 andere modellen bestaande uit de meest belangrijke klinische parameters. Het CT radiomics model presteerde vergelijkbaar met het beste klinische model, wat aanleiding geeft tot verder onderzoek. Door de integratie van beeldvorming, geavanceerde computermodellen zoals radiomics en biomarkers in het NIGT-platform kunnen beslismodellen worden gemaakt voor patiënten met een GBM. Met deze beslismodellen kan de besluitvorming rondom een behandeling voor patiënten met een GBM worden ondersteund en op maat worden gemaakt: personalized medicine.

In deel II is onderzocht op welke wijze de 7 Tesla (T) MRI kan worden geïntegreerd binnen de radiotherapie en worden enkele belangrijke technische aspecten hierbij besproken. MRI-scanners met een ultrahoge veldsterkte hebben een hogere ruimtelijke resolutie in vergelijking met de momenteel klinisch gebruikte $1.5 \mathrm{~T}$ en 3T MRI-scanners. In hoofdstuk 4 wordt een voorbereidende studie beschreven waarin de technische haalbaarheid van de integratie van de 7T MRI in zowel neurochirurgische navigatie software als een radiotherapie planningssysteem is onderzocht. Voor deze studie hebben 3 vrijwilligers een 7 T MRI-scan ondergaan. De scans werden goed verdragen. Het verlies van signaal kon worden geminimaliseerd 
door middel van het gebruik van di-electische kussens en het inlezen en verwerken van de MRI-beelden in de planningsoftware had geen invloed op de kwaliteit van de beelden. Echter, in de nabijheid van holtes gevuld met lucht werden susceptibiliteits artefacten geobserveerd zoals bijvoorbeeld ter plaatse van de frontaalkwab nabij de sinussen. De geometrische onzekerheden veroorzaakt door het MRI-systeem waren na correctie $\leq 2 \mathrm{~mm}$. De conclusie van deze studie is dat $7 \mathrm{~T}$ MRI-beelden veilig, met een hoge kwaliteit en geometrisch betrouwbaar gebruikt kunnen worden in zowel neurochirurgische als radiotherapeutische planningssystemen. De geometrische nauwkeurigheid van 7T MRI is verder onderzocht in hoofdstuk 5. Deze studie beoogde de klinische toepasbaarheid van anatomische 7T MRI te onderzoeken in vergelijking tot $3 \mathrm{~T} \mathrm{MRI}$ en $\mathrm{CT}$ als hulpmiddel voor precisie radiotherapie, door de omvang en locatie van hardware gerelateerde geometrische distorsie te evalueren. Voor deze analyse werd gebruik gemaakt van drie methodes: 1) globale gemiddelde absolute afwijking, 2) lokale gemiddelde absolute afwijking en 3) euclidische afstanden gemeten tussen de "point of interest"-coördinaten. Ondanks het toepassen van geometrische correctiemethoden en tweede en derde orde shimming was systeemgerelateerde geometrische distortie aanwezig in alle 3T en 7T MRI-beelden. Deze nam toe wanneer de afstand van het magnetisch isocentrum groter werd. Een geometrische distortie van $\leq 1 \mathrm{~mm}$ kon worden gegarandeerd nabij het magnetisch isocentrum, maar was groter in de periferie. Dit betekent dat 7T veilig en met klinisch acceptabele hoeveelheden geometrische onzekerheid kan worden toegepast bij hoge-precisie radiotherapie in de centrale hersengebieden. Voor perifeer gelegen laesies is het noodzakelijk om rekening houdende met de geometrische distortie aangepaste planning target volume-marges te hanteren.

In deel III worden innovaties in de behandeling van GBM besproken. Het betreft specifiek een studie waarin de veiligheid van chloroquine als toevoeging aan chemoradiatie bij GBM wordt onderzocht. Chloroquine inhibeert autofagie door de laatste stap van het autofagie proces te blokkeren. Dit resulteert in een vermindering van de hypoxische regio in een tumor, wat de cellen kwetsbaarder maakt voor radiotherapie. In hoofdstuk 6 worden de resultaten van een fase lb studie gepresenteerd, waarin chloroquine wordt toegevoegd aan de radiotherapeutische behandeling met temozolomide bij patiënten met een nieuw vastgesteld GBM. In totaal werden 44 bijwerkingen, welke mogelijk aan chloroquine toe te wijzen zijn, vastgesteld. Hierbij was onder meer sprake van QTc-tijd verlenging, permanent troebel zicht en misselijkheid en/of braken. De mediane overleving voor het totale cohort was 16 maanden. Een dagelijkse dosis van $200 \mathrm{mg}$ chloroquine werd vastgesteld als de maximale veilige dosis, wanneer deze gecombineerd wordt met radiotherapie en temozolomide. EGFRvIll werd geïdentificeerd als potentiele voorspellende biomarker. De vastgestelde beperkte toxiciteit en veelbelovende overleving voor patiënten met een EGFRvIll mutatie geven aanleiding tot verder onderzoek. 
Impact paragraph 


\section{Impact paragraph}

Gliobastoma (GBM) represents the most common and aggressive primary brain cancer in adults, accounting for approximately $75 \%$ of malignant brain tumors. GBM has an estimated annual incidence of around approximately 2 in 10,000 people in the European union. Without treatment the median survival time for these patients is around 4.5 months and even with extensive treatment that includes surgery, chemotherapy and radiation therapy, the median survival is only 15 months. Since the Stupp-protocol has become standard of care after its publication in 2005 , no significant survival improvement has been achieved for this combination treatment comprising radiotherapy and concomitant chemotherapy with temozolomide (TMZ). This outlook needs to be improved and requires three fundamental conditions to be fulfilled:

1. The ability to identify the full extent of this highly invasive tumour. This is impossible with the currently available diagnostic tools.

2. The improvement of the efficacy of the available treatment modalities and the development of new therapeutic options.

3. An improved prediction of the benefits and toxicities of the available treatments for individual patients. Due to the nature of this disease, the ability of both physician and patient to make an informed choice, taking into account the potential treatment outcome and side effects versus quality of life, is an essential part of defining patient tailored treatment plans.

In order to create advancements in these three areas this thesis focussed on the following objectives:

1. Review the current and novel diagnostic imaging methods and techniques used to predict treatment response.

2. Investigate the technical feasibility of incorporating $7 \mathrm{~T} \mathrm{MRI}$ into neurosurgical navigation and radiation treatment planning (RTP) systems, and determine the recommended phase II dose for Chloroquine (CQ) in combination with concurrent chemo(TMZ)-radiotherapy in patients with a newly diagnosed GBM.

3. Investigate the optimization of decision support systems to improve treatment outcome prediction.

This thesis focussed on diagnostic methods and therapeutic interventions by three means: (1) through the analysis of images with radiomics and the "Non-invasive Gliobastoma Testing" (NIGT) platform, (2) through the potential of implementing 7 T MRI in radiotherapy practice, and (3) through the addition of CQ to the standard of care treatment.

In Chapters 2 and 3 decision support systems for GBM were investigated. There is a clear need for improved decision support systems as the number of patients with a GBM is expected to increase the coming years due to the ageing population. 
Moreover, as a growth in therapeutic options is anticipated there is a clear need for treatments that are better tailored to patients' individual tumor and preferences. The availability of increasing amounts of data such as clinical characteristics, semantic features, radiomics and molecular markers enables the development of novel decision support systems through the development of treatment outcome prediction models. These models can be used to estimate probabilities for treatment benefits and risks (i.e. side effects) in individual patients and thereby permit a patient individualized approach. To enable physicians to use all of these tools in actual clinical practice, Chapter 2 describes how the currently available non-invasive prognostic and predictive techniques can be combined together to form a comprehensive decision support tool: the NIGT platform. The goal of this platform is to predict and monitor treatment responses in patients with GBM. It allows physicians to fully capture the complexity and heterogeneity of the tumor and enables selection of the most optimal treatment. As both the diagnostic and therapeutic options for GBM continue to expand, such as the radiomics-based prediction model as described in Chapter 3, these techniques can be integrated in the NIGT platform. In a later stage, the tool can also be modified and be made available to patients. In order to give physicians and patients access to this support system, it will be published on the website https://www.cancerdata.org/ through which it will made publicly available and can be applied during the consultations with radiation oncologists to support the treatment decision-making process. This will assist both physicians and patients to make the best decision for the patient's personal situation, an ability relevant to all cancer patients, but even more so to GBM patients considering the careful balance between quantity- and quality-of-life.

The studies described in Chapters 4 and 5 regarding $7 \mathrm{~T}$ MRI were the first to investigate its possible implementation for improved diagnosis and therapy targeting of GBM into clinical practice. These studies identified susceptibility artefacts and quantified geometrical distortions present in $7 \mathrm{~T} \mathrm{MR}$ images. The studies also showed that there are still some technical challenges to overcome before 7T MRI can be clinically implemented for neuro-oncological RTP. Further research to determine the full clinical value of $7 \mathrm{~T} \mathrm{MRI} \mathrm{for} \mathrm{the} \mathrm{treatment} \mathrm{of} \mathrm{GBM} \mathrm{is} \mathrm{required,} \mathrm{and} \mathrm{the} \mathrm{image}$ quality of $7 \mathrm{~T} \mathrm{MRI}$ needs further improvement. Medical technology companies such as Siemens, together with for instance the ultra-high-field MR imaging center Scannexus B.V. in Maastricht, are working on the latter. The research presented in this thesis demonstrates that the integration of high quality and geometrically reliable 7 T anatomical MR images into neurosurgical navigation and RTP systems is technically feasible and safe. The recommendations from these studies can be implemented within clinical (radiation oncology) practice.

A potentialavenue to improve the efficacy of existing GBM treatments was investigated through the phase I study presented in Chapter 6. There has been mounting preclinical evidence of the efficacy of CQ for autophagy inhibition in cancer. CQ is 
a readily available, generic drug which could be added almost immediately to the standard treatment of GBM with limited toxicity and with minimal financial impact. Although CQ has previously been combined with radiotherapy and chemotherapy in GBM, the presented phase I study is the first to combine CQ with the current standard of care treatment according to the Stupp-protocol. The study demonstrates limited toxicity in combination with this standard treatment and supports further research into the clinical effectiveness of $C Q$.

Based on the (pre)clinical work including the work described in Chapter 6 an orphan drug designation was granted for $C Q$ for the treatment of glioma in the EU in November 2014 (EU/3/14/1377) and in the US in May 2015 (Request number: 15-4750). This provides a 10-year market exclusivity for CQ within the EU and the US. Orphan drugs are generally approved faster than drugs without orphan designation. Furthermore, the orphan designation results in fee reductions for protocol assistance and followup, pre-authorization inspections, new applications for market authorization and post-authorization activities for small and medium-sized enterprises.

Although GBM may appear histologically identical, the prognosis and response to therapy may be dependent on tumor biomarkers. Over the past ten years several predictive and prognostic biomarkers in GBM have been identified such as MGMT promotor methylation and mutations of IDH 1 and 2. Their value has been confirmed in more recent prospective studies. However, patient selection based on tumor biomarkers is only slowly being implemented into clinical practice. Preclinical data have shown that the addition of $C Q$ to standard treatment is potentially most effective in EGFRvIll expressing and hypoxic tumors. The identification of predictive biomarkers may improve patient survival and reduce toxicity in non-responding patients in a cost-effective manner. In the proposed phase II trial as described in the Future Perspectives section of this thesis both the effect of CQ on survival as the value of EGFRvIII in identification of patients who respond to CQ treatment will be established. The phase II study is summarised in the following video: https://youtu.be/H3W2wD8Pjsc. The high unmet need for improved treatment for GBM and the absence of other effective treatment options position CQ close to market introduction.

In summary, the work described in this thesis has explored several new approaches that hold the potential to improve the overall survival in the treatment of patients with GBM. The promising results achieved give rise to further investigate their clinical, scientific and societal impact and judge their value on their respective merits. 
Dankwoord 


\section{Dankwoord}

Het is eindelijk zo ver, mijn proefschrift is af. Het zijn interessante en leerzame jaren geweest met grote hoogte- en dieptepunten. Interessant en leerzaam onderzoek, mooie publicaties, nieuwe baan en nieuw leven, maar ook laatste loodjes die zwaar wegen en moeten accepteren dat je vakgebied je ook privé heel hard kan raken.

Er zijn heel veel mensen die bijgedragen hebben aan deze prestatie, waarvoor ik ze graag wil bedanken.

Allereerst wil ik de patiënten bedanken die hebben deelgenomen aan de fase I studie. Tijdens een enorm zware periode in uw leven heeft $u$ de moed gehad om deel te nemen aan een studie met daarbij het risico dat de zware behandeling nog heftiger werd. Zonder $u$ was deze studie niet mogelijk geweest en ik zal mij tijdens mijn carrière blijven inzetten om de behandeling van het glioblastoom te blijven verbeteren.

Prof. dr. Philippe Lambin: Je was tijdens mijn promotie een eindeloze bron van ideeën en energie. Jouw scherpe opmerkingen gaven vaak net het kleine duwtje in de goede richting. Bedankt voor deze mogelijkheid.

Prof. dr. Dirk de Ruysscher: Hartelijk dank voor het overnemen van de begeleiding binnen MAASTRO, het delen van ongelooflijk veel kennis en het ondersteunen van de laatste loodjes.

dr. Danielle Eekers: Als co-promotor was je mijn steun- en toeverlaat. Jouw enthousiasme en passie werken aanstekelijk. Ik hoop dat we in de toekomst nog lang samen mogen werken en onze vele mooie projecten tot een succes brengen.

Prof. dr. ir. Aswin Hoffmann: Ik ben heel blij dat jij mijn proefschrift hebt willen begeleiden. Jouw kritische blik en prachtige formuleringen hebben mijn proefschrift zo veel beter gemaakt.

$d r$. Ann Hoeben: jouw enorme hoeveelheid kennis en tomeloze inzet voor patiënten zorgden ervoor dat ik met een gerust hart onze proefpersonen durfde te includeren.

De leden van de beoordelingscommissie (Prof. dr. F.J.W. Verhaegen, Prof. dr. Y. Temel, Prof. dr. M. Smits, Prof. dr. M. Lambrecht, dr. F. de Vos): Hartelijk dank voor uw tijd en inzet bij de beoordeling van mijn proefschrift en voor een fijne samenwerking nu en in de toekomst.

StopHersentumoren.n/ en SWOL: jullie zagen toekomst in mijn onderzoeksvoorstellen en zonder jullie vertrouwen en financiële ondersteuning was dit onderzoek niet mogelijk geweest.

Monique Bessems, Nadja Drummen en Anita Botterweck: Dankjulliewel voor jullie enorme toewijding en zorgvuldigheid. Jullie hielden het overzicht, waar ik het regelmatig zoek was. 
John Paulissen en Claudia Offerman: Jullie zijn onmisbaar voor het klinische onderzoek bij MAASTRO. Net zoals de patiënten die jullie dagelijks begeleiden, ben ik jullie erg dankbaar.

Ruud Houben: Menig keer stond ik aan jouw bureau voor advies rondom een statische analyse of een powerberekening. Dankjewel voor het delen van jouw expertise.

Brigitta Baumert: Jouw gedrevenheid voor het onderzoek naar hersentumoren leidde jaren geleden naar mijn eerste academische stapjes in dit onderzoeksgebied.

Alle co-auteurs: Voor zover nog niet benoemd wil ik alle co-auteurs hartelijk danken voor de fijne samenwerking, kritische blikken en goede feedback.

Maikel, het was een plezier om met jou samen te werken, heel veel succes met het afronden van jouw proefschrift. Jurgen, we hebben samen heel wat puntjes op MRls gezet en al dat werk heeft geleid tot 2 mooie publicaties. Succes met je verdere carrière. Kasper, jouw onderzoek vormde de basis voor mijn fase I studie. De fase II studie is er helaas (nog) niet van gekomen. Ik hoop in de toekomst opnieuw samen mooie studies op te kunnen zetten. Tom en Robert Jan, dankjewel voor de prettige samenwerking voor nu en in de toekomst. Ralph and Henry, thank you for sharing your expertise in a way that even a simple clinician like me can understand. Leonard, your patience and skill helped me through stressful times. I look forward to collaborating on future projects.

Neuro-oncologie team (Ann, Christianne, Danielle, Elise, Jan, Karin, Koos, Linda A, Linda J, Martijn, Mieke, Miquel, Monique, Olaf, Onno, Petra, Piet, Rob en Tineke): voor de fijne samenwerking tijdens de afgelopen jaren en in de toekomst.

Marielle: Dankjewel voor de geweldige secretariële ondersteuning de afgelopen maanden.

Yasmine: Mijn boekje ziet er dankzij jou fantastisch uit. Ik ben je ook erg dankbaar dat het door jouw inzet mogelijk was om mijn vader nog een kopie van mijn proefschrift aan te kunnen bieden.

Frank, Piet, Frederik, Danielle Ellen en Karin: Hoewel klinisch werk, onderzoek doen en een jong gezin niet altijd de perfecte combinatie was, kon ik op jullie steun en luisterend oor rekenen. Ik werk met veel plezier in ons team en hoop dat we nog lang samen mooie dingen kunnen doen.

Alle andere collega's bij MAASTRO: Ik werk inmiddels al vele jaren met plezier bij MAASTRO. Dankjulliewel voor de interesse en morele ondersteuning door de jaren heen.

Veerle, Mimmi, Madieke, Linda, Ghislaine, Yvonne, Charlotte en Iris: al sinds de middelbare school dikke vriendinnen. Ik geniet enorm van onze jaarlijkse uitstapjes en hoop dat we deze nog lang samen blijven doen.

Catie, Cyriel, Erik, Renée, Iris, Lucien, Myra, Sander, Niels, Alexandra, Piter en Sabrina: Dankjulliewel voor de jarenlange vriendschap, spelletjes avonden en al het plezier 
dat we samen hebben beleefd. Hoewel we niet allemaal meer in Maastricht wonen, is het ieder Sinter-Kerst-en-Nieuw-diner weer alsof er niets is veranderd.

Iris en Veerle (para-nimfen): door dik en dun staan jullie voor mij klaar. Ik kan geen betere vriendinnen wensen.

Kees, Renée, Tim, Wendy, Joke en Maaike: dankjulliewel voor de interesse en steun afgelopen jaren. Ik bof maar met zo'n familie.

Mark: We hebben al veel samen meegemaakt. Wij begrijpen elkaar, een blik is vaak genoeg. Ik ben blij dat jij mijn grote broer bent en ik weet dat ik altijd op jou kan rekenen.

Bram en Bas: Lieve Bram, straks heb ik weer alle tijd voor jou. Ik hou van jou tot aan het maantje en terug. Lieve Bas, zonder jouw geduld en liefde was dit nooit gelukt. Jullie zijn het allerbelangrijkste in mijn leven. Ik hou van jullie beide met heel mijn hart.

Papa: Geneeskunde met een natuurkundig tintje. Zoals ik als klein meisje op jouw schouders zat, kon ik ook tijdens het schrijven van mijn thesis op jou bouwen. Jij bent tijdens het leven mijn grote voorbeeld geweest. Hoewel we ver van elkaar woonden, wist ik dat ik altijd bij jou terecht kon. Je blijft altijd in mijn gedachten. 
Curriculum Vitae 


\section{Curriculum Vitae}

Inge Compter werd geboren op 7 februari 1988 in Hoogeveen. Na het afronden van haar VWO-opleiding aan het Lorentz Casimir Lyceum in Eindhoven (2000 - 2006, Cum Laude), begon zij in 2006 aan haar studie geneeskunde aan de Universiteit van Maastricht. Aansluitend aan het behalen van haar arts-examen in 2012 ging zij in opleiding tot radiotherapeut-oncoloog bij MAASTRO in Maastricht. Vanaf augustus 2015 startte Inge officieel met haar promotieonderzoek naar het verbeteren van de behandeling en beeldvormende modaliteiten voor patiënten met een glioblastoom onder begeleiding van prof. P. Lambin en prof. D. De Ruysscher bij MAASTRO clinic. In augustus 2017 rondde zij haar specialisatie af en vanaf april 2018 werkt Inge als radiotherapeut-oncoloog bij MAASTRO met als aandachtsgebieden hoofdhalstumoren, sarcomen, dermatologie, palliatie en de neuro-oncologie.

Op 27 mei 2017 is Inge met Bas Tielemans getrouwd en precies 2 jaar later werden zij de gelukkige ouders van Bram.

\section{Subsidies}

- Brains Unlimited Pioneer Fund / Stichting Wetenschappelijk Onderwijs Limburg (SWOL), 2013, t.w.v. 9075,- euro voor onderzoek naar ultra-high field magnetic resonance imaging.

- Stichting StopHersentumoren.nl, 2015, t.w.v. 45.854,- euro voor de fase I chloroquine studie. 
List of publications 


\section{List of publications}

Compter I, Verduin M, Shi Z, Woodruff HC, Smeenk RJ, Rozema T, Leijenaar RTH, Monshouwer R, Eekers DBP, Hoeben A, Postma AA, Dekker A, De Ruysscher D, Lambin $\mathrm{P}$, Wee L. Deciphering the glioblastoma phenotype by Computed Tomography radiomics. Radiother Oncol. $2021 \mathrm{Jul}$

Eekers DBP, Di Perri D, Roelofs E, Postma AA, Dijkstra J, Ajithkumar T, Alapetite C, Bromstrand M, Burnet NG, Calugaru V, Compter I, Coremans IEM, Harrabi S, lannalfi A, Klaver YLB, Lambrecht M, Méndez Romero A, Paulsen F, Timmermann B, Vitek P, van der Weide HL, Whitfield GA, Witt Nyström P, Zindler J, De Ruysscher D, Langendijk J, Weber DC, Troost EGC, MD on behalf of the taskforce "European Particle Therapy Network" of ESTRO. Update of the EPTN atlas for CT- and MR-based (3T and 7T) contouring in Neuro-Oncology. Radiother Oncol. $2021 \mathrm{Jul}$

In 't Ven L, Compter I, van Eijsden K, Zindler J, Swinnen A, de Ruysscher D, Rozema T, Troost EGC, Eekers DBP. A validated model to predict alopecia in brain tumor patients receiving cranial irradiation. Submitted to Radiotherapy \& Oncology 2021

't Ven L, Roelofs E, Mesías MC, Compter I, Klaver YLB, Smeenk RJ, Janssens GO, Kaanders JHAM, Fajardo RD, Oldenburger F, de Ruysscher D, Troost EGC, Eekers DBP. The ROCOCO Performance Scoring System translates dosimetric differences into clinically relevant endpoints: comparing IMPT to VMAT in an example pilocytic astrocytoma dataset. Clin Transl Radiat Oncol. 2021 Feb

Verduin M, Primakov S, Compter I, Woodruff HC, van Kuijk SMJ, Ramaekers BLT, te Dorsthorst $M$, Revenich EGM, ter Laan $M$, Pegge SAH, Meijer FJA, Beckervondersandforth J, Speel EJ, Kusters B, de Leng WWJ, Anten MM, Broen MPG, Ackermans L, Schijns OEMG, Teernstra O, Hovinga K, Vooijs MA, Tjan-Heijnen VCG, Eekers DBP, Postma AA, Lambin P, Hoeben A. Prognostic and predictive value of integrated qualitative and quantitative magnetic resonance imaging analysis in glioblastoma. Cancers. 2021 Feb

Jaspers JPM, Méndez Romero A, Wiggenraad R, Compter I, Eekers DBP, Nout RA, van den Bent M. Pattern of failure in IDH mutated, low grade glioma after radiotherapy - Implications for margin reduction. Radiother Oncol. 2020 Nov

Compter I, Eekers DBP, Hoeben A, Rouschop KMA, Reymen B, Ackermans L, Beckervordersantforth J, Bauer NJC, Anten MM, Wesseling P, Postma AA, De Ruysscher $\mathrm{D}$, Lambin P. Chloroquine combined with concurrent radiotherapy and temozolomide for newly diagnosed glioblastoma: a phase IB trial. Autophagy. 2020 Sep. 
Koemans WJ, Larue RTHM, Kloft M, Ruisch JE, Compter I, Riedl RG, Heij LR, van Elmpt W, Berbée M, Buijsen J, Lambin P, Sosef MN, Grabsch HI. Lymph node response to chemoradiotherapy in oesophageal cancer patients: relationship with radiotherapy fields. Esophagus. 2020 Sep.

Weis J, Wirtz MA, Tomaszewski KA, Hammerlid E, Arraras Jl, Conroy T, Lanceley A, Schmidt $H$, Singer S, Pinto M, Alm El-Din M, Compter I, Holzner B, Hofmeister D, Chie WC, Harle A, Flechtner HH, Bottomley A; EORTC Quality of Life Group. Sensitivity to change of the EORTC quality of life module measuring cancer-related fatigue (EORTC QIQ-Fa12): Results from the international psychometric validation. Psychooncology. 2019 Aug.

Walsh S, de Jong EEC, van Timmeren JE, Ibrahim A, Compter I, Peerlings J, Sanduleanu S, Refaee T, Keek S, Larue RTHM, van Wijk Y, Even AJG, Jochems A, Barakat MS, Leijenaar RTH, Lambin P. Decision Support Systems in Oncology. JCO Clin Cancer Inform. 2019 Feb.

Peerlings J, Compter I, Janssen F, Wiggins C, Mottaghy F, Lambin P, Hoffmann A. Characterizing geometrical accuracy in clinically optimized $7 T$ and $3 T$ MR images for high-precision radiation treatment of brain tumours. Physics and Imaging in Radiation Oncology. 2019 Jan.

Eekers DB, in 't Ven L, Roelofs E, Postma A, Alapetite C, Burnet NG, Calugaru V, Compter I, Coremans IEM, Høyer M, Lambrecht M, Nyström PW, Méndez Romero A, Paulsen F, Perpar A, de Ruysscher D, Renard L, Timmermann B, Vitek P, Weber DC, van der Weide HL, Whitfield GA, Wiggenraad R, Troost EGC; “European Particle Therapy Network" of ESTRO. The EPTN consensus-based atlas for CT- and MR-based contouring in neuro-oncology. Radiother Oncol. $2018 \mathrm{Jul}$.

Verduin M, Compter I, Steijvers D, Postma AA, Eekers DBP, Anten MM, Ackermans L, Ter Laan M, Leijenaar RTH, van de Weijer T, Tjan-Heijnen VCG, Hoeben A, Vooijs M. Noninvasive Glioblastoma Testing: Multimodal Approach to Monitoring and Predicting Treatment Response. Dis Markers. 2018 Jan.

Jutten B, Keulers TG, Peeters HJM, Schaaf MBE, Savelkouls KGM, Compter I, Clarijs R, Schijns OEMG, Ackermans L, Teernstra OPM, Zonneveld MI, Colaris RME, Dubois L, Vooijs MA, Bussink J, Sotelo J, Theys J, Lammering G, Rouschop KMA. EGFRvIll expression triggers a metabolic dependency and therapeutic vulnerability sensitive to autophagy inhibition. Autophagy. 2018 Jan. 
Weis J, Tomaszewski KA, Hammerlid E, Ignacio Arraras J, Conroy T, Lanceley A, Schmidt H, Wirtz M, Singer S, Pinto M, Alm El-Din M, Compter I, Holzner B, Hofmeister D, Chie WC, Czeladzki M, Harle A, Jones L, Ritter S, Flechtner HH, Bottomley A; EORTC Quality of Life Group. International Psychometric Validation of an EORTC Quality of Life Module Measuring Cancer Related Fatigue (EORTC QLQ-FA12). J Natl Cancer Inst. 2017 May.

Zindler JD, Jochems A, Lagerwaard FJ, Beumer R, Troost EGC, Eekers DBP, Compter I, van der Toorn PP, Essers M, Oei B, Hurkmans CW, Bruynzeel AME, Bosmans G, Swinnen A, Leijenaar RTH, Lambin P. Individualized early death and long-term survival prediction after stereotactic radiosurgery for brain metastases of non-small cell lung cancer: Two externally validated nomograms. Radiother Oncol. 2017 May.

Compter I*, Peerlings J*, Eekers D. B.P, Postma A. A, Ivanov D, Wiggins C. J, Kubben P, Küsters B, Wesseling P, Ackermans L, Schijns O. E. M.G, Lambin P, Hoffmann A. L. Technical feasibility of integrating 7 Tesla anatomical MRI in image-guided radiotherapy of glioblastoma: a preparatory study. MAGMA. 2016 Jun.

Lambin P, Zindler J, Vanneste BG, De Voorde LV, Eekers D, Compter I, Panth KM, Peerlings J, Larue RT, Deist TM, Jochems A, Lustberg T, van Soest J, de Jong EE, Even AJ, Reymen B, Rekers N, van Gisbergen M, Roelofs E, Carvalho S, Leijenaar RT, Zegers CM, Jacobs M, van Timmeren J, Brouwers P, Lal JA, Dubois L, Yaromina A, Van Limbergen EJ, Berbee M, van Elmpt W, Oberije C, Ramaekers B, Dekker A, Boersma LJ, Hoebers F, Smits KM, Berlanga AJ, Walsh S. Decision support systems for personalized and participative radiation oncology. Adv Drug Deliv Rev. 2016 Jan.

Müller K, Henke G, Pietschmann S, van Gool S, De Vleeschouwer S, von Bueren AO, Compter I, Friedrich, C, Matuschek C, Klautke G, Kortmann RD, Hundsberger T, Baumert BG. Re-irradiation or re-operation followed by dendritic cell vaccination? Comparison of two different salvage strategies for relapsed high-grade gliomas by means of a new prognostic model. J Neurooncol. 2015 Jun.

Müller K, Henke G, Compter I, von Bueren AO, Friedrich C, Janssens G, Kramm CM, Hundsberger T, Paulsen F, Kortmann RD, Zwiener I, Baumert BG. External validation of a prognostic model estimating the survival of patients with recurrent high-grade gliomas after reirradiation. Pract Radiat Oncol. 2014 Nov.

Compter I, Zaugg K, Houben RM, Dings JT, Bosmans G, Buescher C, Anten MM, Baumert BG. High symptom improvement and local tumor control using stereotactic radiotherapy when given early after diagnosis of meningioma. A multicentre study. Strahlenther Onkol. 2012 Oct.

*indicates equal contribution 



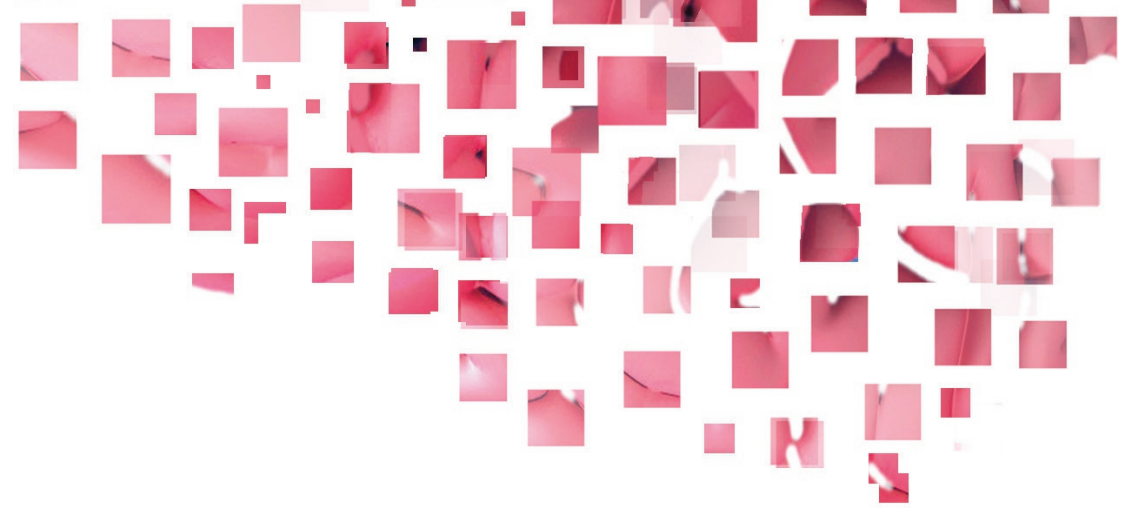

